Pion Inelastic Scattering from ${ }^{20} \mathrm{Ne}$

Michael Burlein*

+Guest Scientist at Los Alamos. Department of Physics, University of Pennsylvania, Philadelphia, PA 19104.

\title{
MASTER do
}

DSTRIBUTION OF THIS DBCUMENT IS UNLIMITED 


\section{Acknowledgments}

I am grateful for the assistance and support of many people throughout my undergraduate and graduate education. First and foremost there is my advisor, Terry Fortune, who provided support, encouragement, advice, and ideas in my work in nuclear physics. Thanks to all the people who showed great patience in teaching me how run an experiment and analyze data, especially Ron Giiman, Kalvir Dhuga, and John Zumbro. I am indebted to all of my collaborators, in particular those who helped out on the experiment: Walter Anos, Tor Ekenberg, Ashtosh Kotwal, Peter Kutt, Jim Silk, John O'Donnell, Brent Boyer, A. Fuentes, Kevin Johnson, Sung Yoo, C. Fred Moore, Kalvir Dhuga, John Zumbro, Doug Watgon, and Shaul Mordechai, who managed to get the LAMPF PAC to allow this experiment to run. Many thanks to the LAMPF staff and to the MP-10 staff, especially Chris Morris, Steve Greene, and Dick Boudrie, who taught me about EPICS. I would also like to thank many of the people at the University of Pennsylvania Tandem for their support and assistance, in particular Jim Silk, John O'Donnell, and Peter Kutt. 
Pion Inelastic Scattering from ${ }^{20} \mathrm{Ne}$

Michael Burlein

\begin{abstract}
Angular distributions for ${ }^{20} \mathrm{Ne}\left(\pi^{*}, \pi^{\prime \prime}\right)$ were measured on the Energetic Pion Channel and Spectrometer (EFICS) at the Clinton P. Anderson Meson Physicg Facility (LAMPF). Data were taken with both $\pi^{+}$and $\pi^{-}$cver an angular range of $12^{\circ}$ to $90^{\circ}$ for $T_{\pi}=180 \mathrm{MeV}$ and with $\pi^{+}$from $15^{\circ}$ to $90^{\circ}$ for $T_{\pi}=120 \mathrm{MeV}$. The data were analyzed using both the distorted-wave impulse approximation (DWLA) and the coupled-channels impulse approximation (CCLA) with collective transition densities. In addition, micruscopic transition densities were used in the DWIA analysis for states in the lowest rotational bands. The transitions to the 6.73- $\mathrm{MeV} \mathrm{O}^{+}$and several $1^{-}$states, including the states at $5.79 \mathrm{MeV}$ and $8.71 \mathrm{MeV}$, were studied using several models ior the transition density. Strong evidence for the importance of two-step routes in pion inelastic scattering was seen in several angular distributions, including the $5.79-\mathrm{MeV}^{-}$, the first three $4^{+}$states, and the 8.78- $\mathrm{MeV}^{+}$.
\end{abstract}




\section{Table of Contents}

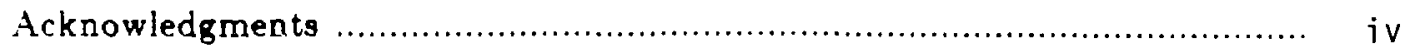

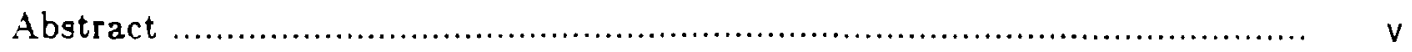

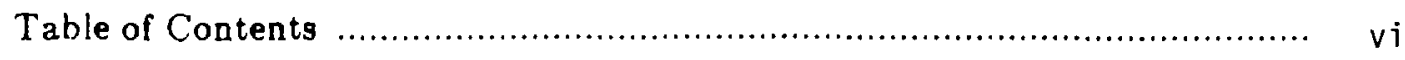

List of Tables ........................................................................ $\mathrm{i} \mathrm{i}$

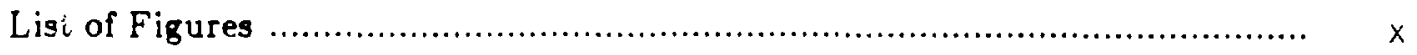

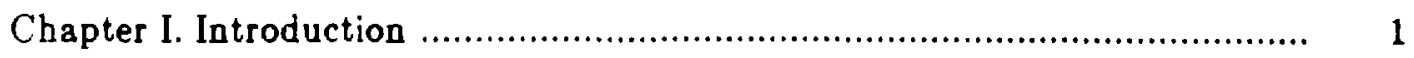

I-1. Pion Inelastic Scattering .................................... 2

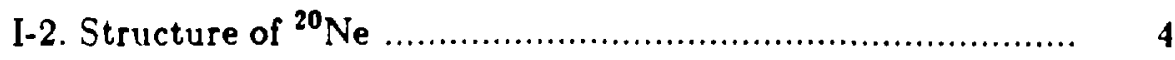

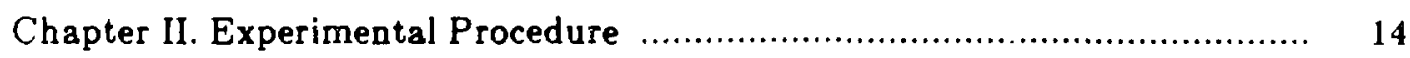

II-1. LAMPF Accelerator and Primary Beam ..................... 14

II-2. EPICS Channel .............................................. 17

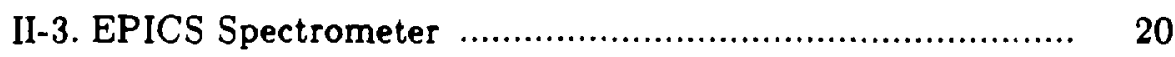

II-4. EPICS Cooled Gas Target ......................................... 24

II-5. Analysis of Events .......................................... 24

II-6. Normalizations ............................................ 27

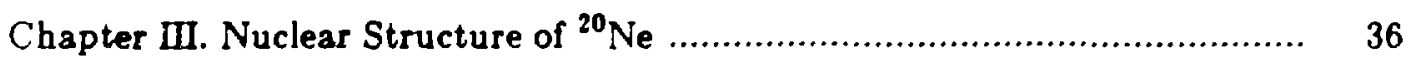

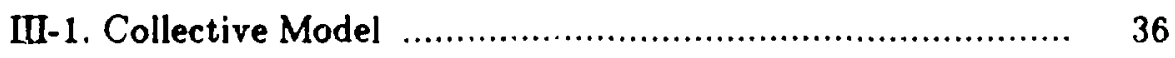

III-2. Shell Model ..................................................... 47

III-3. Other Models ................................................. 63

Chapter IV. Pi-Nucleus Scattering Theory .................................... 67

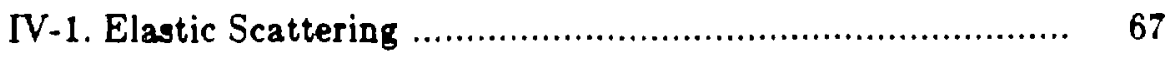

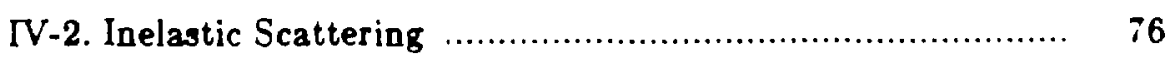




$$
-v i i-
$$

Chapter V. Analysis and Results ............................................. 86

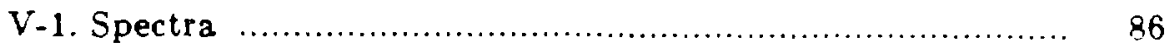

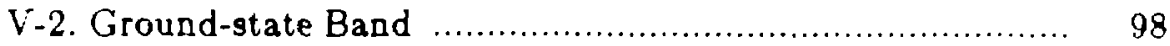

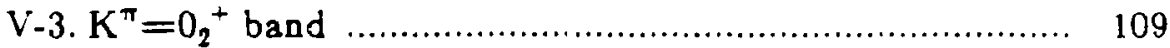

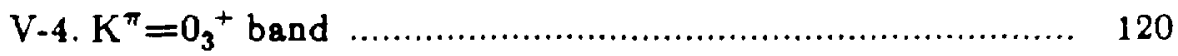

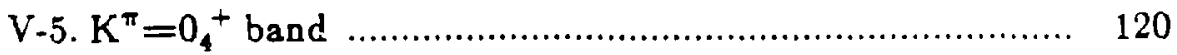

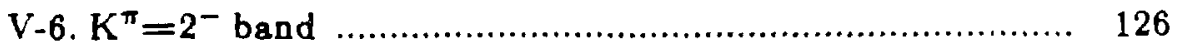

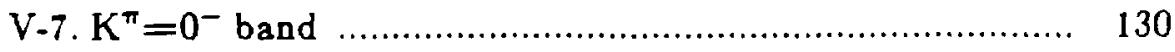

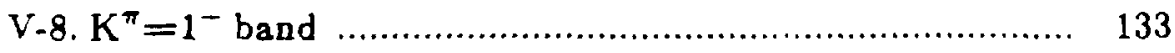

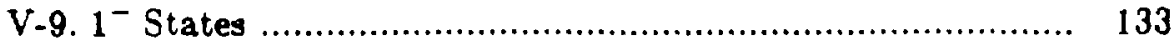

V-10. Microscopic Calculations ................................. 147

Chapter VI. Conclusions ........................................................... 169

Appendix 1. High Excitation Peaks ............................................ 176

Appendix 2. Pion Inelastic Scattering to the Low-lying Broad $2^{+}$in ${ }^{20} \mathrm{Ne}$.

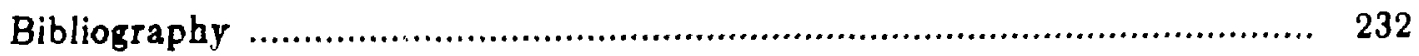




\section{List of Tables}

I-1. Properties of pions. .....................................................................

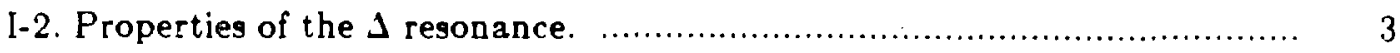

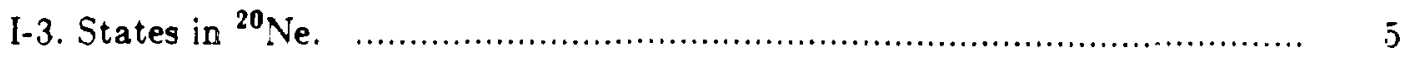

I-4. Ground-state charge densities from elastic electron scattering. $\ldots \ldots \ldots \ldots \ldots . . . . .11$

I-5. Experimental measurements of the deformation of ${ }^{20} \mathrm{Ne} . \ldots \ldots \ldots \ldots \ldots \ldots \ldots . . . . . . . .11$

I-6. Low-lying states seen in inelastic $\mathrm{p}$ or $\alpha$ scattering. ............................ 11

I-7. Inelastic electron scattering data for $0^{+}$states in ${ }^{20} \mathrm{Ne}$........................ 12

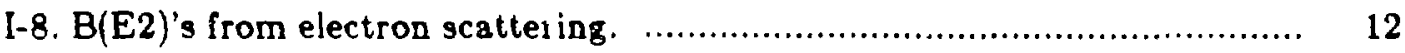

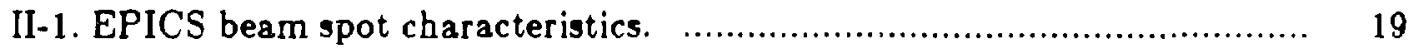

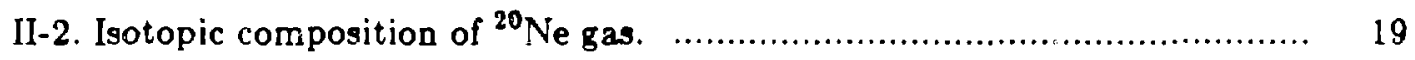

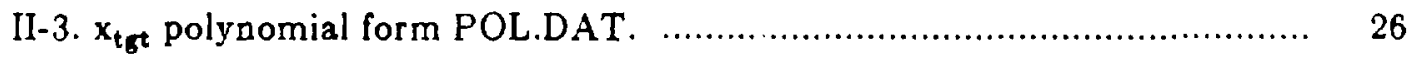

II-4. Some quantities calculated from POL.DAT. ...................................... 29

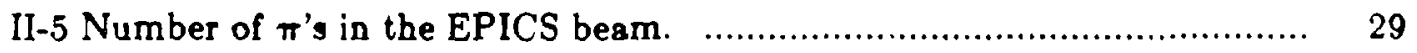

III-1. Energy dependence of states in the rotational hands in ${ }^{30} \mathrm{Ne} . \quad \ldots \ldots \ldots \ldots . . . . . .43$

III-2. $\left|\left\langle\chi_{K}\left|Q_{20}{ }^{\prime}\right|_{X_{K}}\right\rangle\right|$ for the ground state band in ${ }^{20} \mathrm{Ne} . \ldots \ldots \ldots \ldots \ldots \ldots . . . . . . . . . . . .43$

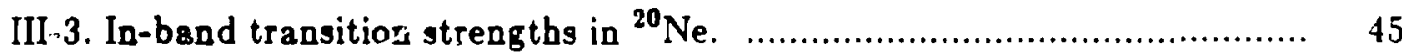

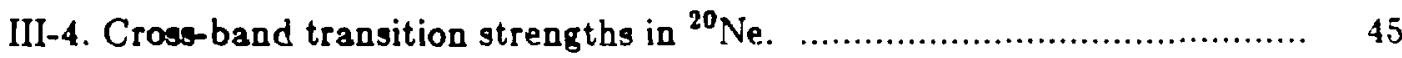

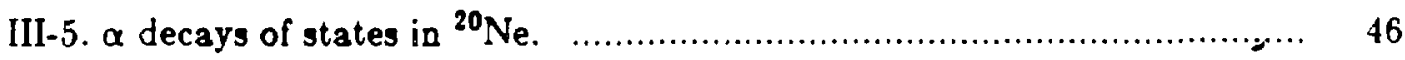

III-6. Comparison of (sd) ${ }^{4}$ calculated B(E2)'s with experimental B(E2)'s.

III-7. Energy dependence of states in the rotational bands from the (sd) 
calculation.

ill-8. $\left|<\chi_{K}\right| Q_{20}{ }^{\prime} \chi_{K}>\mid$ for the $K^{\pi}=0^{+}$bands in ${ }^{20} \mathrm{Ne}$ from the $(\mathbf{s d})^{4}$ calculation.

III-9. Comparison of ZBM calculated $B(E \lambda)$ 's with experimental $B(E \lambda)$ 's.

III-10. Energy dependence of states in the rotational bands from the ZBM calculation.

III-11. $\left|<\chi_{K}\right| Q_{20}{ }^{\prime} \chi_{\chi_{K}}>\mid$ for the $K^{\pi}=0^{+}$bands in ${ }^{20} \mathrm{Ne}$ from the $Z B M$ calculation.

III-12. $B(E 2)$ 's for transitions for negative parity bands in the ZBM calculation.

V-1. EPICS gas cell composition.

V-2. Peaks in ${ }^{20} \mathrm{Ne}$.

V-3. Ground-state transition strengths extracted with DWIA calculations.

V-5. Transition strengths extracted with DWLA for peaks involving $l=0,1$ transitions.

V-6. Effective charges extracted for ZBM and (sd) ${ }^{4}$ model spaces.

VI-1. Comparison of $B(E l t)$ 's from pion scattering with other data.

VI-2. Comparison of B(E/1)'s from pion scattering with theory.

A1-1. Ground-state transition strengths extracted with DWLA calcula-

tions.

A1-2. Possible isovector transitions in ${ }^{20} \mathrm{Ne}\left(\pi, \pi^{\prime}\right)$. 


\section{List of Figures}

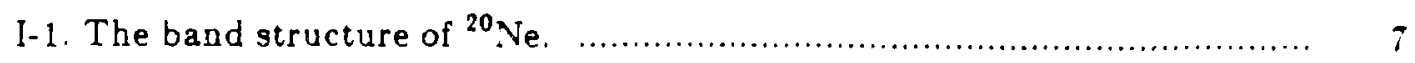

I-2. Structure of the positive-parity bands of ${ }^{20} \mathrm{Ne}$ in the Nilsson model. ...... 8

1-3. Structure of the negative-parity bands of ${ }^{20} \mathrm{Ne}$ in the Nilsson model.

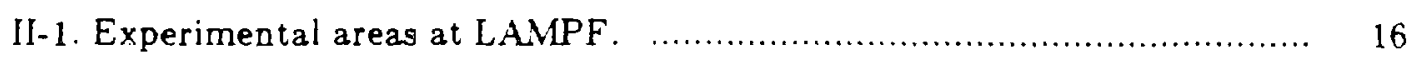

II-2. Schematic of the EPICS channel. ........................................ 18

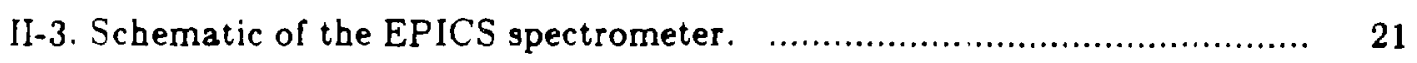

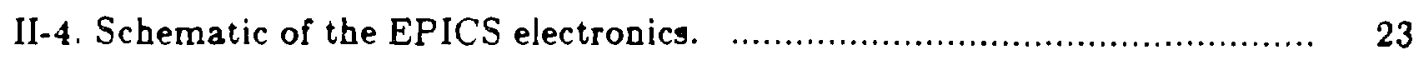

II-5. Diagram of the EPICS cooled gas cell. ....................................... 25

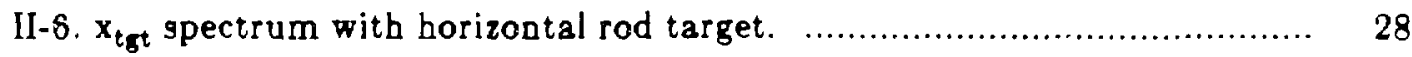

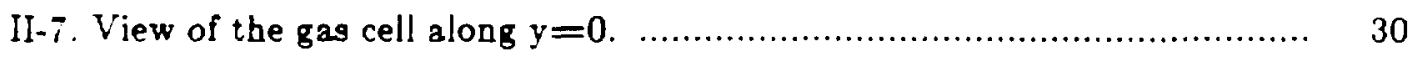

II-8. ${ }^{1} \mathrm{H}(\pi, \pi)^{1} \mathrm{H}$ angular distributions calculated using the phase shifts of

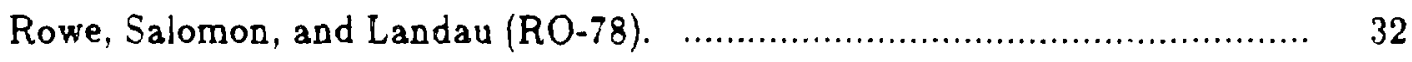

II-9. Schematic of the sections of the target and gas cell the spectrometer

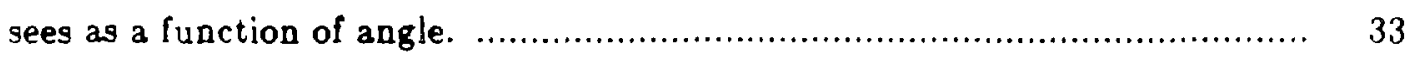

II-10. Angular dependence of the normalizations. .............................. 34

II-11. Acceptance scan used in this experiment. ................................... 35

III-1. Excitation energies of the states in ${ }^{20} \mathrm{Ne}$ plotted versus $\mathrm{J}(\mathrm{J}+1) . \ldots \ldots \ldots . \quad 41$

III-2. Approximate level pattern from the shel! model. .......................... 49

III-3. Energies calculated in the (sd) ${ }^{4}$ model space and the observed ener-

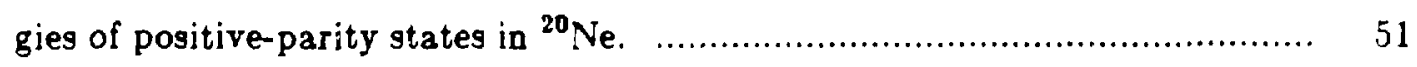

III-4. Energies calculated in the $(s d)^{4}$ model space plotted versus $J(J+1)$. 
III-5. Energies calculated in the ZBM model space and the observed energies of positive-parity states in ${ }^{20} \mathrm{Ne}$.

III-6. Energies calculated in the ZBM model space and the observed energies of negative-parity states in ${ }^{20} \mathrm{Ne}$.

III- 7 . Energies calculated in the ZBM model space plotted versus $J(J+1)$.

III-8. Approximate single-particle levels for the Nilsson Hamiltonian.

V-1. Fitted missing mass spectrum for ${ }^{20} \mathrm{Ne}\left(\pi^{+}, \pi^{+\prime}\right)$ at $30^{\circ}$ with $\mathrm{T}_{\pi}=180$

$\mathrm{MeV}$ from -2 to $8 \mathrm{MeV}$ excitation.

V-2. Same as Figure V-1 but for 4 to $8 \mathrm{MeV}$ excitation. ............................. 89

V-3. Same as Figure V-1 but for 7 to $11 \%$ MeV excitation. .......................... 90

V-4. Same as Figure V-1 but for 10 多 to $13 \frac{1}{2} \mathrm{MeV}$ excitation. ...................... 91

V-5. Same as Figure V-1 but for $12 \frac{1}{2}$ to $17 \frac{1}{2} \mathrm{MeV}$ excitation. .................... 92

V-6. Empty target missing mass histogram for $180-\mathrm{MeV} \pi^{+}$at $30^{\circ}$. ............ 93

V-7. Angular distributions for the elastic peak. ........................................ 99

V-9. Angular distributions for the $1.63-\mathrm{MeV}^{+}$and the $4.25-\mathrm{MeV}_{4}^{+}$.

V-9. Angular distributions for the 8.78- $\mathrm{MeV}^{+}$.

101

$V-10$. Couplings used in the CCLA calculations for states in the $\mathrm{K}^{\pi}=0_{1}^{+}$ band.

$\mathrm{V}-11$. CCLA calculations to the $4.25-\mathrm{MeV} 4^{+}$and the $8.78-\mathrm{MeV}^{+}$. 107

$\mathrm{V}-12$. CCLA calculations to ihe $4.25-\mathrm{MeV}^{+}$and the $8.78-\mathrm{MeV}^{+}$. 108

$\mathrm{V}-13$. Angular distributions for the $7.42-\mathrm{MeV}^{+}$and the $9.99-\mathrm{MeV}^{+}$.

V-14. Couplings used in the CCIA calculations for the $9.99-\mathrm{MeV}^{+}$state. 


$$
-x i i-
$$

V-15. CCLA calculations to the 9.99-MeV $4^{+}$.

$\mathrm{V}-16$. Couplings used in the CCIA calculations for the $6.73-\mathrm{MeV}^{+}$and

$7.42-\mathrm{MeV} 2^{+}$states.

V-17. CCIA calculations to the $7.42-\mathrm{MeV}^{+}$for $180 \mathrm{MeV}^{+}$incident

beam.

$\mathrm{V}-18 . l=0$ transition densities used for the $6.73-\mathrm{MeV}^{+}$.

V-19. Angular distribuitions for the 6.73- $\mathrm{MeV}^{+}$.

$\mathrm{V}-20$. CCLA calculations to the $6.73-\mathrm{MeV} \mathrm{O}^{+}$.

V-21. Angular distributions for the $7.83-\mathrm{MeV}^{+}$and the $9.03-\mathrm{MeV}^{+}$.

V.22. CCIA calculations to the $9.03-\mathrm{MeV}_{4}^{+}$.

$\mathrm{V}$-23. Angular distributions for the $9.00-\mathrm{MeV}^{+}$and the $10.80-\mathrm{MeV}^{+}$.

V-24. Angular distributions for the $1.63-\mathrm{MeV}^{+}$and the $9.00-\mathrm{MeV}^{+}$.

$\mathrm{V}-25$. Angular distributiong for the $5.62-\mathrm{MeV}^{-}$and the $8.45-\mathrm{MeV}^{-}$peak.

$\mathrm{V}$-26. Angular distributions for the $8.45-\mathrm{MeV}$ peak.

$\mathrm{V}-27$. CCLA calculations to the 8.45-MeV $5^{-}$.

V-28. Angular distributions for the $7.16-\mathrm{MeV} 3^{-}$and the $10.26-\mathrm{MeV}$

peak.

V-29. CCIA calculations to the $10.26-\mathrm{MeV}^{-}$.

$\mathrm{V}$-30. Angular distributions for the $10.41-\mathrm{MeV} \mathrm{3}^{-}$

V-31. I=1 transition densities used for transitions to $1^{-}$states.

V-32. Angular distributiong for the $5.79-\mathrm{MeV}_{1}^{-}$and the $8.71-\mathrm{MeV}^{-}$. 


\section{$-x i j i-$}

V-33. Angular distributions for the $11.22-\mathrm{MeV}^{-}$and the $11.48-\mathrm{MeV}^{-}$.

$V^{r}$-34. Angular distributions for the $13.72-\mathrm{MeV}^{-}$and the $15.59-\mathrm{MeV}^{-}$.

$V-35$. Angular distributions for the $11.48-\mathrm{MeV}$ peak.

$V$-36. Seme as Figure $V-35$ except the $l=1$ calculations use the transition density of HA-81.

$\mathrm{V}-37$. Angular distributions for the $14.88 \mathrm{-MeV}$ peak.

$\mathrm{V}$-38. Angular distributions for the $17.27-\mathrm{MeV}$ peak.

V-39. Same as Figure V-38 except the $I=1$ calculations use the transition

density of HA-81.

V-40. Angular distributions for the $8.71-\mathrm{MeV}^{-}$.

V-41. Couplings used in the CCLA calculations for the $5.79-\mathrm{MeV}^{-}$state.

$\mathrm{V}-42$. CCLA calculations to the 5.79- $\mathrm{MeV}^{-}$.

V-43. CCLA calculations to the $5.79-\mathrm{MeV}^{-}$

V-44. CCLA calculations to the 5.70-MeV $1^{-}$.

V-45. Angular distributions for the 6.73- $\mathrm{MeV}^{+}$.

$\mathrm{V}-46$. Angular distributions for the $6.73-\mathrm{MeV}^{+}$.

V-47. Angular distributions for the 5.79-MeV and the 8.71-MeV ${ }^{-}$

states.

$\mathrm{V}-48$. Angular distributions for the 4.97-MeV $2^{-}$state.

V-49. Angular distributions for the $1.63-\mathrm{MeV}$ and the $7.42-\mathrm{MeV}^{+}$

states.

$\mathrm{V}-50$. Angular distributions for the $1.63-\mathrm{MeV}$ and the $7.42-\mathrm{MeV}^{-}$ 
V-51. Same as Figure V-50 but for the $7.83-\mathrm{MeV}$ state.

$\mathrm{V}$-52. Angular distributions for the $5.62 \mathrm{M} \mathrm{M} \mathrm{V}$ and the $7.16-\mathrm{MeV}^{-}$

states.

V-53. Same as Figure V-52 but for the $5.62-\mathrm{MeV}$ and $10.41-\mathrm{MeV}$ states.

V-54. Angular distributions for the 4.25-MeV and the $9.99-\mathrm{MeV}^{+}$

states.

V-55. Angular distributions for the $4.25-\mathrm{MeV}^{+}$state.

V-56. Angular distributions for the $9.03-\mathrm{MeV}$ and $9.99-\mathrm{MeV}^{+}$states.

V-57. Same as Figure V-56 except assuming the $4^{+'} \mathrm{~s}$ in the shell-model

calculation switch as they do experimentally

VI-1. Comparison of $B(E)$ extracted with pion scattering and electromag-

netic probes.

VI-2. Comparison of $B(E \eta$ extracted with pion scattering and shell-model

calculations.

Ai-1. Angular distributions for peaks between 8.71 and $11.22 \mathrm{MeV}$ in excitation which could be fit with a single $l$.

A1-2. Same as Figure A1-1 except the graphs are $180-\mathrm{MeV}^{-}$data.

184

A1-3. Same as Figure A1-1 except the graphs are $120-\mathrm{MeV}^{+}$data.

185

A1-4. Same as Figure A1-1 but for states between 11.64 and $12.03 \mathrm{MeV}$ in

excitation.

A1-5. Same as Figure A1-4 except the graphs are $180-\mathrm{MeV} \pi^{-}$data. $\ldots . . . . . . \quad 187$

A 1-6. Same as Figure $A 1-4$ except the graphs are $120-\mathrm{MeV} \pi^{+}$data.

A1-7. Same as Figure A1-1 but for states between 12.38 and $12.85 \mathrm{MeV}$ in excitation. 


$$
-X V-
$$

A1-8. Same as Figure A1-7 except the graphs are $180-\mathrm{MeV}^{-}$data.

A1-9. Same as Figure Al-7 except the graphs are $120-\mathrm{MeV}^{+}$data.

A1-10. Same as Figure A1-1 but for stales between 13.08 and $13.72 \mathrm{MeV}$

in excitatirin.

A1-11. Same as Figure A1-10 except the graphs are 180-MeV $\pi^{-}$data.

A1-12. Same as Figure $A 1-10$ except the graphs are $120-\mathrm{MeV} \pi^{+}$data.

A1-13. Same as Figure A1-1 bi for states between 13.94 and $14.35 \mathrm{MeV}$

in excitation.

A1-14. Same as Figure A1-13 except the graphs are $180-\mathrm{MeV} \pi^{-}$data.

A1-15. Same as Figure Al-13 except the graphs are $120-\mathrm{MeV} \pi^{+}$data.

A1-16. Same as Figure A1-1 but for states between 14.44 and $15.59 \mathrm{MeV}$

in excitation.

A1-17. Same as Figure A1-16 except the graphs are $180-\mathrm{MeV} \pi^{-}$data.

A1-18. Same as Figure A1-16 except the graphs are $120-\mathrm{MeV} \pi^{+}$data.

A1-19. Same as Figure A1-1 but for states between 15.91 and $17.13 \mathrm{MeV}$

in excitation.

A1-20. Same as Figure A1-19 except the graphs are 180-MeV $\pi^{-}$data.

A1-21. Same as Figure A1-19 except the graphs are $120-\mathrm{MeV} \pi^{+}$data. 
A1-22. Angular distributiong for peaks between 9.88 and $10.90 \mathrm{MeV}$ in excitation which could not be well fit with a single $l$.

A1-23. Same as Figure A1-22 except i he graphs are $180-\mathrm{MeV}^{-}$data.

A1-24. Same as Figure A1-22 except the graphs are $120-\mathrm{MeV}^{+}$data.

A1-25. Same as Figure A1-22 but for states between 11.08 and $11.83 \mathrm{MeV}$

in excitation.

A1-26. Same as Figure A1-25 except the graphs are $18 l_{-}-\mathrm{MeV} \pi^{-}$data.

A1-27. Same as Figure A1-25 except the graphs are $120-\mathrm{MeV} \pi^{+}$data.

A1-28. Same as Figure A1-22 but for states between 12.22 and $14.88 \mathrm{MeV}$

in excitation.

A1-29. Same as Figure A1-28 except the graphs are 180-MeV $\pi^{-}$dats.

A1-30. Same as Figure A1-28 except the graphs are 120-MeV $\pi^{+}$data.

A1-31. Same as Figure A1-22 but for states between 15.13 and 15.36 MeV

in excitatios.

A1-32. Same as Figure A1-31 except the graphs are 18G-MeV $\pi^{-}$data.

A1-33. Same as Figure A1-31 except the graphs are $120-\mathrm{MeV} \pi^{+}$data.

A1-34. Same as Figure A1-22 but for states between 15.74 and $16.59 \mathrm{MeV}$ in excilation. 


$$
-x \vee i i-
$$

A1-35. Same as Figure A1-34 except the graphs are $180-\mathrm{MeV} \pi^{-}$data.

Al-36. Same as Figure A1-34 except the graphs are $120-\mathrm{MeV} \pi^{+}$data. 


\section{Introduction}

Nuclear physics can be described as a study of the structure of atomic nuclei and their interactions. Experimentalists use reactions whose mechanisms are understood to study states in nuclei whose structure is not understood and states in nuclei whose structure is underntood to study reaction mechanisms that, are not understood. Different reactions are dependent on different aspects of nuclear structure, or, to look at it the other way, different reactions give different information on the structure of the nuclear states. Elastic-scattering reactions reveal information about the distribution of nucleons in the ground state; inelastic-scattering reactions give information about the transition density between the initial and final states. One- and two-particle transfer reactions can be used to determine the parentage of states. Studying $\gamma$ decays of states gives data on the electromagnetic matrix elements between the states. This thesis is a study of the ${ }^{20} \mathrm{Ne}\left(\pi^{*}, \pi^{*}\right)$ reaction at $T_{\pi}=120 \mathrm{MeV}$ and $180 \mathrm{MeV}$. The primary purpose of this study was to improve the understanding of coupled-channels effects in pion inelastic scattering. 


\section{I-1. Pion inelastic scattering}

Nuclear physics has found inelastic scattering of elementary particles at intermediate and high energies to be a very useful cool in studying nuclear structure. Electron scattering has been very successful in measuring proton transition densities in nuclei. The electron-nucleus interaction is just the well-known electromagnetic interaction between the electron and the protons in the nucleus. Unfortunately, it is difficult to get any information on neutrons from electron scattering. To learn about the structure of the neutrons in a nucleus it is necessary to use particles which interact with the strong nuclear force, e.g $\pi$ 's, protons, $\alpha$ 's, etc. In principle using protons or $\alpha$ particles in conjunction with electron scattering data should make it possible to remove the contributions of the protons and learn something about the neutrons. This can be very difficult, in particular for $\alpha$ particles, as the $\alpha$-nucleus interaction must be determined. The impulse approximation can be used for protons if the energy is high enough. The impulse approximation assumes that the interaction of the projectile with the nucleons in the nucleus is the same as the interaction of the projectile with a free nucleon. This approximation should be valid if the beam energy is significantly higher than the binding energy of the nucleons, about $50 \mathrm{MeV}$.

The pion is the lightest strongly interacting particle. Some properties of the pion are given in Table 1-1. Pion scattering is a particularly good tool to study both the neutrons and the protons in nuclei because $\sigma\left(\pi^{+} p\right)=\sigma\left(\pi^{-} n\right)$ and $\sigma\left(\pi^{+} \mathrm{n}\right)=\sigma\left(\pi^{-} \mathrm{p}\right)$, so going from $\pi^{+}$to $\pi^{-}$inverts the sensitivity to neutrons and protons. The dominant feature of the pi-nucleon interaction around $180 \mathrm{MeV}$ is the $\Delta$ resonance, the first excited state of the nucleon. Some properties of the $\Delta$ are given in Table $1-2$. On the resonance $\sigma\left(\pi^{+} p\right) \approx \theta \sigma\left(\pi^{-} p\right)$ and $\sigma\left(\pi^{+} n\right) \approx \frac{1}{9} \sigma\left(\pi^{-} n\right)$. Therefore by scattering pions at resonance it is particularly 
TABLE I-1.

$\begin{array}{ccc}\begin{array}{cc}\text { Properties of pions } \\ \pi^{+}\end{array} & \pi^{0} & \pi^{-} \\ +1 & 0 & -1 \\ 0^{-} & 0^{-} & 0^{-} \\ 1,1 & 1,0 & 1,-1 \\ 139.6 & 13 \mathrm{~s}^{-0} & 139.6 \\ 2.6 \times 10^{-8} & 8.7 \times 10^{-17} & 2.6 \times 10^{-8} \\ 780.4 & 2.6 \times 10^{-0} & 780.4 \\ \mu^{+} v & \frac{1}{\sqrt{2}}(u \bar{u}-\mathrm{dd}) & \bar{u} \mathrm{~d} \\ u d & & \end{array}$

1.) Data from PA-86.

TABLE I-2.

Properties of the $\Delta$ resonancel.

\begin{tabular}{|c|c|c|c|c|}
\hline & $\mathrm{A}^{++}$ & $\Delta^{+}$ & $\Delta^{0}$ & $s^{-}$ \\
\hline Charge & +2 & +1 & $0_{+}$ & -1 \\
\hline$J^{\pi}$ & $\underline{3}$ & $\underline{3}^{+}$ & $\underline{3}^{+}$ & $\underline{3}$ \\
\hline$J$ & $3^{2} 3$ & $3^{2} 1$ & $3^{2} 1$ & $\underline{3}^{2}-3$ \\
\hline$T, T_{\imath}$ & & $\frac{7}{2}, \frac{7}{2}$ & $\frac{-1}{2},-\frac{\pi}{2}$ & $\overline{2}$ \\
\hline Mass (MeV) & $1230-1234$ & $1230-1234$ & $1230-1234$ & $1230-1234$ \\
\hline$\Gamma(\mathrm{MeV})$ & $110-120$ & $110-120$ & $110-120$ & $110-127$ \\
\hline $\begin{array}{l}\text { Primary decay } \\
\text { Quark structure }\end{array}$ & $\begin{array}{c}N_{\pi}, N \gamma \\
\text { uuu }\end{array}$ & $\begin{array}{l}N \pi, N \gamma \\
\text { uud }\end{array}$ & $\begin{array}{c}N_{\pi, N y} \\
\text { udd }\end{array}$ & $\begin{array}{c}N \pi, N \gamma \\
\text { ddd }\end{array}$ \\
\hline
\end{tabular}

1.) Data from PA-86. 
easy to find differences between the neutrons and protons in a nucleus.

The unique properties of the pion have been used to study a large number of nuclei in the past fifteen years. The distorted-wave impulse approximation (DWLA) has been used with geat success for strong collective transitions (BL84a.OA-87,SE-81). Transition strengths extracted with a DWLA analysis usually agree with strengths known from other experiments, generally $\gamma$-decay work. However, for weak transitions a DWLA analysis of pion scattering data often yields strengths that are much larger than those known from other data. This may be the result of coupled-channels effects or microscopic effects. "... 3 the $\Delta$ resonance the pion is a very strongly interacting particle and has a very short mean free path, about $0.4 \mathrm{fm}$ at $160 \mathrm{MeV}(\mathrm{ER}-88 \mathrm{~b})$. The pion sees only the surface of the transition density; so if, for example, the transition density has a node in the interior the pion will never see it and the DWLA analysis will give a matrix element which is too large.

Because the pion is very strongly interacting on the $\Delta$ resonance, two-step contributions might also be very important. If both the final state and the ground state have a strong matrix element coupling them to some intermediate state, generally the first excited $2^{+}$in even-even nuclei, then the reaction might proceed by first exciting that intermediate state and then going to the final state. If the strength for this route is comparable to the direct route or some other two-step route then those routes add coherently and can interfere destructively or constructively.

\section{I-2. Structure of ${ }^{20} \mathrm{Ne}$}

${ }^{20} \mathrm{Ne}$ is a nucleus which has been studied very extensively; the latest compilation (AJ-87) lists over 200 states. Some information for the lower excited states is 
TABLE I-3.

\begin{tabular}{|c|c|c|c|c|}
\hline \multicolumn{5}{|c|}{ States in ${ }^{20} \mathrm{Ne}^{11}$. } \\
\hline$E_{x}(\mathrm{MeV})$ & $\Delta E(\mathrm{keV})$ & $\mathrm{J}^{\pi} ; \mathrm{T}$ & $\mathrm{K}^{\pi}$ & $\tau$ or $\Gamma$ \\
\hline 0.0 & & $0^{+}: 0$ & $0_{1}^{+}$ & stable \\
\hline $1.6 \succeq 3674$ & 0.015 & $2^{+} ; 0$ & $0_{1}^{+}$ & $1.05 \pm 0.06$ psec \\
\hline 4.2477 & 1.1 & $4^{+} ; 0$ & $0_{1}^{+}$ & $93 \pm 9 \mathrm{fsec}$ \\
\hline 4.96651 & 0.20 & $2^{-} ; 0$ & $2^{-}$ & $4.8 \pm 0.5 \mathrm{psec}$ \\
\hline 5.6214 & 1.7 & $3^{-} ; 0$ & $2^{-}$ & $200 \pm 50 \mathrm{fsec}$ \\
\hline 5.788 & 2.6 & $1^{-} ; 0$ & $0^{-}$ & $0.028 \pm 0.003 \mathrm{keV}$ \\
\hline 6.725 & 5 & $0^{+} ; 0$ & $\mathrm{O}_{2}^{+}$ & $19.0 \pm 0.9 \mathrm{keV}$ \\
\hline 7.004 & 3.6 & $4^{-} ; 0$ & $2^{-}$ & $400 \pm 90$ fsec \\
\hline 7.1563 & 0.5 & $3^{-} ; 0$ & $0^{-}$ & $8.2 \pm 0.3 \mathrm{keV}$ \\
\hline 7.191 & 3 & $0^{+} ; 0$ & $\mathrm{O}_{3}^{+}$ & $3.4 \pm 0.2 \mathrm{keV}$ \\
\hline 7.4219 & 1.2 & $2^{+} ; 0$ & $\mathrm{O}_{2}^{+}$ & $15.1 \pm 0.7 \mathrm{keV}$ \\
\hline 7.829 & 2.4 & $2^{+} ; 0$ & $\mathrm{O}_{3}^{+}$ & $2 \mathrm{keV}$ \\
\hline 8.453 & 4 & $5^{-} ; 0$ & $2^{-}$ & C $213 \pm 0.004 \mathrm{keV}$ \\
\hline 8.7 & & $0^{+} ; 0$ & $0_{4}^{+}$ & $>800 \mathrm{keV}$ \\
\hline 8.708 & 7 & $1^{-} ; 0$ & & $2.1 \pm 0.8 \mathrm{keV}$ \\
\hline 8.7776 & 2.2 & $6^{+} ; 0$ & $0_{1}^{+}$ & $0.11 \pm 0.02 \mathrm{keV}$ \\
\hline 8.8 & & $2^{+} ; 0$ & $0_{4}^{+}$ & $>800 \mathrm{keV}$ \\
\hline 8.82 & & $\left(5^{-}\right) ; 0$ & & $<1 \mathrm{keV}$ \\
\hline 8.854 & 5 & $1^{-} ; 0$ & $1^{-}$ & $19 \mathrm{keV}$ \\
\hline 9.031 & 7 & $4^{+} ; 0$ & $\mathrm{O}_{3}^{+}$ & $3 \mathrm{keV}$ \\
\hline 9.116 & 3 & $3^{-} ; 0$ & & $3.2 \mathrm{keV}$ \\
\hline 9.318 & 2 & $\left(2^{-}\right) ; 0$ & & \\
\hline 9.487 & 5 & $2^{+} ; 0$ & & $29 \pm 15 \mathrm{keV}$ \\
\hline 9.873 & 4 & $3^{+} ; 0$ & & \\
\hline 9.935 & 12 & $\left(1^{+}\right) ; 0$ & & $<35$ fsec \\
\hline 9.990 & 8 & $4^{+} ; 0$ & $\mathrm{O}_{2}^{+}$ & $155 \pm 30 \mathrm{keV}$ \\
\hline 10.262 & 5 & $5^{-} ; 0$ & $0^{-}$ & $145 \pm 40 \mathrm{keV}$ \\
\hline 10.274 & 3 & $2^{+} ; 1$ & & $\leq 0.3 \mathrm{keV}$ \\
\hline 10.406 & 5 & $3^{-} ; 0$ & $1^{-}$ & $80 \mathrm{keV}$ \\
\hline 10.553 & 5 & $4^{+} ; 0$ & & $16 \mathrm{keV}$ \\
\hline 10.584 & 5 & $2^{+} ; 0$ & & $24 \mathrm{keV}$ \\
\hline 10.609 & 6 & $6^{-} ; 0$ & $2^{-}$ & $23 \pm 7$ fsec \\
\hline 10.694 & 6 & $4^{-}, 3^{+} ; 0$ & & \\
\hline 10.80 & 75 & $4^{+} ; 0$ & $0_{4}^{+}$ & $350 \mathrm{keV}$ \\
\hline 10.840 & 6 & $3^{-} ; 0$ & & $45 \mathrm{keV}$ \\
\hline 10.843 & 4 & $2^{+} ; 0$ & & $13 \mathrm{keV}$ \\
\hline 10.884 & 3 & $3^{+} ; 1$ & & $<30$ fsec \\
\hline 10.917 & 6 & $3^{+} ; 0$ & & \\
\hline 10.97 & 120 & $0^{+} ; 0$ & $0_{5}^{+}$ & $580 \mathrm{keV}$ \\
\hline
\end{tabular}

1.)Data from AJ-87. All states below $11 \mathrm{MeV}$ are listed. 
listed in Table I-3. ${ }^{20} \mathrm{Ne}$ is well described with a rotational model, which is described in much greater detail in Chapter 3. All states below $8.5 \mathrm{MeV}$ have been placed in a rotational band, as shown in Figure I-1.

The structure of the lowest bands in ${ }^{20} \mathrm{Ne}$ is reasonably well understood, as shown in Figures $\mathrm{I}-2$ and $\mathrm{I}-3$ (FO-72b,FO-73a,FO-77). The first two $\mathrm{K}^{\pi}=0^{+}$ bands, with bandheads at 0.0 and $6.7 \mathrm{MeV}$, are primarily (sd) ${ }^{4}$; the third, with its bandliead at $7.2 \mathrm{MeV}$, is believed to be mostly $(\mathrm{sd})^{8}(\mathrm{p})^{-4}$; and the fourth, with its bandhead at $=8$ ine $\vec{V}$, is thought to be either $(f p)^{4}$ or $(f p)^{2}(s d)^{2}$. Evidence for this structure comes from particle transfer reactions. All but the third $0^{+}$band are seen in $\alpha$ stripping reactions on ${ }^{10} \mathrm{O}$, e.g. ${ }^{10} \mathrm{O}\left({ }^{\circ} \mathrm{Li}, \mathrm{d}\right),(\mathrm{FO}-73 \mathrm{~b})$, or ${ }^{10} \mathrm{O}(\alpha, \alpha)$, (MC$60, \mathrm{HA}-72)$, or ${ }^{10} \mathrm{O}(\alpha, \gamma)$, (PE-64,DI-7i,RO-71a,AL-72,RO-71b,LI-67). The first three bands are seen in 8-paricicle stripping on ${ }^{12} \mathrm{C}$, e.g. ${ }^{12} \mathrm{C}\left({ }^{12} \mathrm{C}, \alpha\right)$, (MI-71,FO74a,FO-74b,BA-72,ME-75). All but the fourth are seen in $\alpha$ pickup on ${ }^{24} \mathrm{Mg}$, e.g. ${ }^{24} \mathrm{Mg}\left(\mathrm{d},{ }^{\circ} \mathrm{Li}\right)$, (FO-78,CO-72). One-particle transfer data, (BE-75), also support these conclusions, although it is necessary to assume mixing between the second and fourth bands to explain the presence of states from the fourth band in the ${ }^{10} \mathrm{~F}\left({ }^{3} \mathrm{He}, \mathrm{d}\right)$ data (FO-72a,FO-76), and the $\alpha$ widths of members of the second band.

The structure of the three lowest negative-parity bands is also known from particle-transfer data. The $\mathrm{K}^{\pi}=2^{-}$and $\mathrm{K}^{\pi}=1^{-}$bands are believed to be primarily $(s d)^{5}(p)^{-1}$ and the $K^{\pi}=0^{-}$band is probably $(f p)^{1}(g d)^{3}$. The $2^{-}$band is very weak in $\left({ }^{3} \mathrm{He}, \mathrm{d}\right)$, while the $0^{-}$band is strong $(\mathrm{BE}-75)$. In ${ }^{22} \mathrm{Ne}(\mathrm{p}, \mathrm{t})$ and ${ }^{21} \mathrm{Ne}(\mathrm{p}, \mathrm{d})$ the $2^{-}$is strong (FA-71,HE-72,HO-70). The 8.84-MeV $1^{-}$is strong in $(\mathrm{d}, \mathrm{t})$ which implies that this state is the bandhead of the $1^{-}$band with the same Nilsson orbits as the $2^{-}$band. In $\alpha$ pickup from ${ }^{24} \mathrm{Mg}$ the $2^{-}$band is stiongly excited and the $0^{-}$ band is weakly excited. However, in $(d, L i)$ the reaction mechanisin was not simply direct pickup of an $\alpha$ since both the $2^{-}$and $4^{-}$states were excited, which is 
States in ${ }^{20} \mathrm{Ne}$

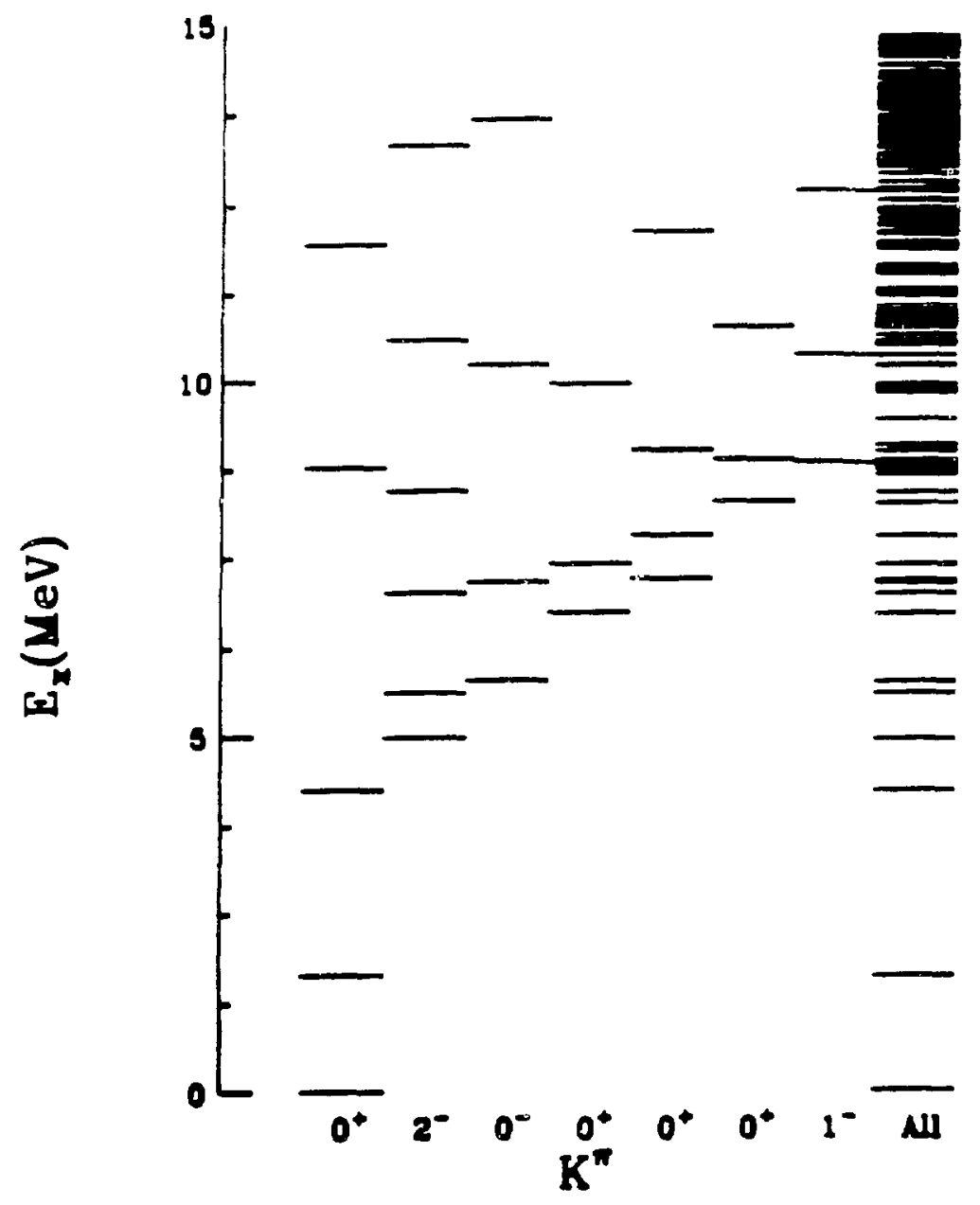

Figure I-1.

The band structure of ${ }^{20} \mathrm{Ne}$. 


$$
\begin{aligned}
& 1 / 2-[330]- \\
& 3 / 2+[211]- \\
& 1 / 2+[220] \frac{\pi \pi \nu \nu}{1 / 2-[101] \frac{\pi \pi \nu \nu}{\text { i.3. }}}
\end{aligned}
$$$$
\pi \pi \nu \nu
$$$$
\frac{\pi \pi \nu \nu}{0.72 u e v}
$$

$$
\begin{aligned}
& 1 / 2-[330] \\
& 3 / 2+[211] \approx \pi \nu v \\
& 1 / 2+[220] \pi \pi \nu \nu \\
& 1 / 2-[101] \frac{}{7.20 \mathrm{MoV}}
\end{aligned}
$$

Figure 1-2.

Structure of the positive-parity bands of ${ }^{20} \mathrm{Ne}$ in the Nilseon model. 


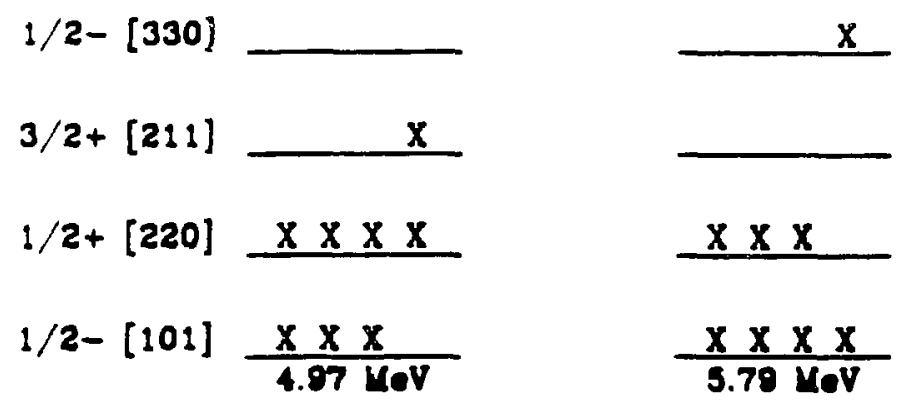

$1 / 2-[330]$

$3 / 2+[211]$

$1 / 2+[220] \times \times \times x$

$1 / 2-[101] \frac{X \times X}{8.86 \mathrm{KoV}}$

Figure 1-3.

Structure of the negative-parity bands of ${ }^{20} \mathrm{Ne}$ in the Nilsoon model. 
prohibited under the selection rules for a direct one-step reaction.

Because ${ }^{20} \mathrm{Ne}$ is a gas and does not form chemical compounds easily, it is difficult to make a ${ }^{20} \mathrm{Ne}$ target. In spite of this difficulty, ${ }^{20} \mathrm{Ne}$ has been studied extensively with elastic and inelastic scattering with a wide variety of projectiles: electrons, (MI-72,SI-73), protons, (OD-59,OD-60,SC-62,SW-69,SW-73,SW-74,SW76,BL-84b,BL-88), anti-protons, (BA-86), alphas, (SE-58,EI-62,SP-65,KO-65,RE71,RE-72,SP-70), etc. Some parameterizations of the ground-state charge density from elastic electron scattering are given in Table 1-4. Analysis of inelastic proton-scattering and alpha-scattering data show that the ground-state band has both a quadrupole and a hexadecapole deformation (SW-69,SW-73,SW-74,SW76,BL-84b,SP-65,RE-71,SP-70,RE-72). Some results of these studies are shown in Table I-5. Several states not in the ground-state band have been seen in the inelastic scattering of protons and alphas, (SC-62,SW-76,BL-88,SE-58,E1-62,SP-65,KO65), and these are listed in Table I-6. However, there has been little success fitting the transitions to states not in the ground-state band (BL-88).

Inelastic electron scattering has been used to study states below $8 \mathrm{MeV}$ in excitatiou, (MI-72,SI-73), in particular the $0^{+}$states at $6.72 \mathrm{MeV}$ and $7.20 \mathrm{MeV}$, (MI-72,SI-73). Both of these stat,es were seen and fitted with a phenomenological transition density of the form

$$
\rho_{\mathrm{tr}}=\left[\sum_{j=0}^{3} a_{j}\left(\frac{r}{b}\right)^{2 j}\right] \exp \left(-\frac{r^{2}}{b^{2}}\right) .
$$

The results of this analysis are given in Table I-7. The $a_{j}$ 's were not reported in either paper so, because terms up to $r^{*}$ were used, the $a_{j}$ 's are not uniquely determined. The results of the analysis of other states seen are given in Table I-8. One anomaly noted was that the $7.42-\mathrm{MeV}^{+}$state had a form factor which looked more like an E4 than an E2 transition (MI-72). 
TABLE I-4.

Ground-state charge densities from elastic electron scattering ${ }^{1)}$.

$\begin{array}{ccccc}\text { Model }^{2)} & c(\mathrm{fm}) & z(\mathrm{fm}) & w(\mathrm{fm}) & \mathrm{q}\left(\mathrm{fm}^{-1}\right)^{3)} \\ 2 \mathrm{pF} & 2.805 \pm 0.015 & 0.571 \pm 0.005 & & 0.22-1.04 \\ 2 \mathrm{pF} & 2.740 \pm 0.046 & 0.572 \pm 0.017 & & 0.21-1.12 \\ 3 \mathrm{pF} & 2.791 \pm 0.009 & 0.698 \pm 0.005 & -0.168 \pm 0.008 & 0.49-1.80\end{array}$

1.) Data from DE-87

2.) $2 \mathrm{pF}$ is a two-parameter Fermi distribution. $3 \mathrm{pF}$ is a three-parameter Fermi distribution.

${ }^{3 .}$ The range in $q$ that was fitted.

\section{TABLE $1-5$.}

Experimental measurements of the deformation of ${ }^{20} \mathrm{Ne}$.

$\begin{array}{cccc}\beta_{2} & \beta_{1} & \text { Reaction } & \text { Reference } \\ +0.47 & +0.25 & \left(p, p^{\prime}\right) & \text { BL-84b } \\ +0.47 \pm 0.04 & +0.28 \pm 0.05 & \left(p, p^{\prime}\right) & \text { SW-73 } \\ +0.35 \pm 0.01 & +0.11 \pm 0.01 & \left(\alpha, \alpha^{\prime}\right) & \text { RE-71 } \\ 0.42 & 0.10 & \left(\alpha, \alpha^{\prime}\right) & \text { SP-65 }\end{array}$

TABLE I-6.

Low-lying states seen in inelastic $p$ or $\alpha$ scattering ${ }^{1)}$.

$\begin{array}{lcc}E_{\mathrm{x}}(\mathrm{MeV}) & \left(\alpha, \alpha^{\prime}\right) & \left(p, p^{\prime}\right) \\ 0.0 & \vee & V \\ 1.63 & V & V \\ 4.25 & V & V \\ 4.97 & V & V \\ 5.62 & V & V \\ 5.79 & V & V \\ 7.16 & V & V \\ 7.42 & V & \\ 8.45 & V & \\ 8.78 & V & \\ 10.26 & V & \end{array}$

1.) Data from OD-59,OD-60,SC-62,SW-69,SW-76,BL-88,SE-58,EI-62,SP-65, and KO-65. 
TABLE I-7.

$\begin{array}{ccc}\text { Inelastic electron scattering data for } 0^{+} \text {states in }{ }^{20} \mathrm{Ne} . \\ \mathrm{E}_{\mathrm{x}}(\mathrm{MeV}) & \mathrm{M}\left(\mathrm{F}^{2}\right) & \mathrm{R}_{\mathrm{tr}} \mathrm{A}^{-\frac{1}{3}} \\ 6.72 & 7.37 \pm 1.97 & 2.11 \pm 0.48 \\ 6.72 & 5.85 \pm 1.5 & 2.21 \\ 7.20 & 6.90 \pm 1.44 & 1.69 \pm 0.52 \\ 7.20 & 5.70 \pm 2.00 & \end{array}$

TABLE I-8.

$B(E 2)$ 's from electron scattering.

$\begin{array}{ccc}E_{\mathrm{x}} & \mathrm{B}(\mathrm{E} \lambda)(W . U .) & \mathrm{B}(\mathrm{E} \lambda)\left(\mathrm{e}^{2} \mathrm{fm}^{4}\right) \\ 1.63 & 17 . \pm 2 . & 274 \pm 32 \\ 7.42 & 0.13 \pm 0.03 & 2.1 \pm 0.5 \\ 7.83 & 0.83 \pm 0.13 & 13.4 \pm 2.1\end{array}$


Because of its well-known rotational structure and because many of the matrix elements between its states are measured. ${ }^{20} \mathrm{Ne}$ was chosen to study twostep contributions to pion inelastic scattering. In rotational nuclei in-band transitions are usually strong and out-of-band transitions tend to be inbibited. The matrix elements between the ground state and the first $2^{+}$and between the ground state and the first $3^{-}$are known to be large enough that they should be strongly excited in pion scattering. Many states in ${ }^{30} \mathrm{Ne}$ have strong matrix elements to these states and weak matrix elements to the ground state, and therefore are very good candidates for a two-step route to be important.

This dissertation is divided into six chapters. The second chapter discusses the experimental procedure. The third and fourth chapters discuss some introductory nuclear structure and reaction theory. The fifth chapter covers the analysis of the data. The sixth chapter presents the conclusions of this work. 


\section{Experimental Procedure}

There are only a few pion beams available in the world, in the United States in Los Alamos, New Mexico, in Canada at TRIUMPF in Vancouver, British Columbia, and in Switzerland at PSI (formerly SIN). The data presented in this experiment are the result of experiment 959U, run at the Clinton P. Anderson Meson Physics Facility at Los Alamos (LAMPF) on the Energetic Pion Channel and Spectrometer (EPICS). There are three pion beams available at LAMPF: EPICS, the Low Energy Pion channel (LEP), and the Pion and Particle Physics channel $\left(\mathrm{P}^{3}\right)$.

\section{II-1. LAMPF accelerator and primary beam}

The accelerator at LAMPF is a linear accelerator (linac) which accelerates protons to $800 \mathrm{MeV}$. Three beams are available at LAMPF: $\mathrm{H}^{+}, \mathrm{H}^{-}$, and polarized $\mathrm{P}^{-}$. The primary proton beam, $\mathrm{H}^{+}$, is used to create the secondary beams of mesons. The negative ion beams, $\mathrm{H}^{-}$and $\mathrm{P}^{-}$, are used in the study of proton induced reactions and are available in a variety of energies. Both beams are 
accelerated simultaneously by the alternating electric fields in the accelerator's if cavities.

The accelerator begins with three injector systems, one for each available beam. Each injector includes an ion source and a Cockcroft-Walton accelerator which accelerates the particles to $750 \mathrm{keV}$. The particles then enter a drift-tube linac which is $62 \mathrm{~m}$ long. It is a series of four vacuum tanks in which an alternating electric field with a frequency of $201.25 \mathrm{MHz}$ is set up. In this stage of the accelerator the particles reach $100 \mathrm{MeV}$. The final stage of the accelerator is a side-coupled-cavity linac, which was invented at Los Alamos. This section is operated at $805 \mathrm{MHz}$ and accelerates the protons to their final energy, $800 \mathrm{MeV}$. Lower energy $\mathrm{H}^{-}$and $\mathrm{P}^{-}$beams are achjeved by turning off sections in this part of the accelerator.

When the beam leaves the accelerator it enters the beam switchyard where the negative ion beams are split from the proton beam and sent to the appropriate experimental area. Figure II-1 diagrams the experimental areas at LAMPF. The negative ion beams go to either Line $D$ or Areas $B$ and $C$. The primary proton beam continues to Area $A$, first to a thin target, usually thorium or uranium. The neutron rich nuclei that are produced in this target are analyzed in the Time-OfFlight Isochronous Spectrometer (TOFI). The beam then reaches the A-1 production target where pion beams for LEP and EPICS are produced. Next it comes to the A-2 target where a pion beam for $\mathrm{P}^{3}$ is produced and a muon beam is produced for the Stopped Muon Channel (SMC). Finally the proton beam reaches the isotope production and radiation-effects facility and the beam stop. Pions decaying in the beam stop produce neutrinos which are used to study neutrino interactions. 


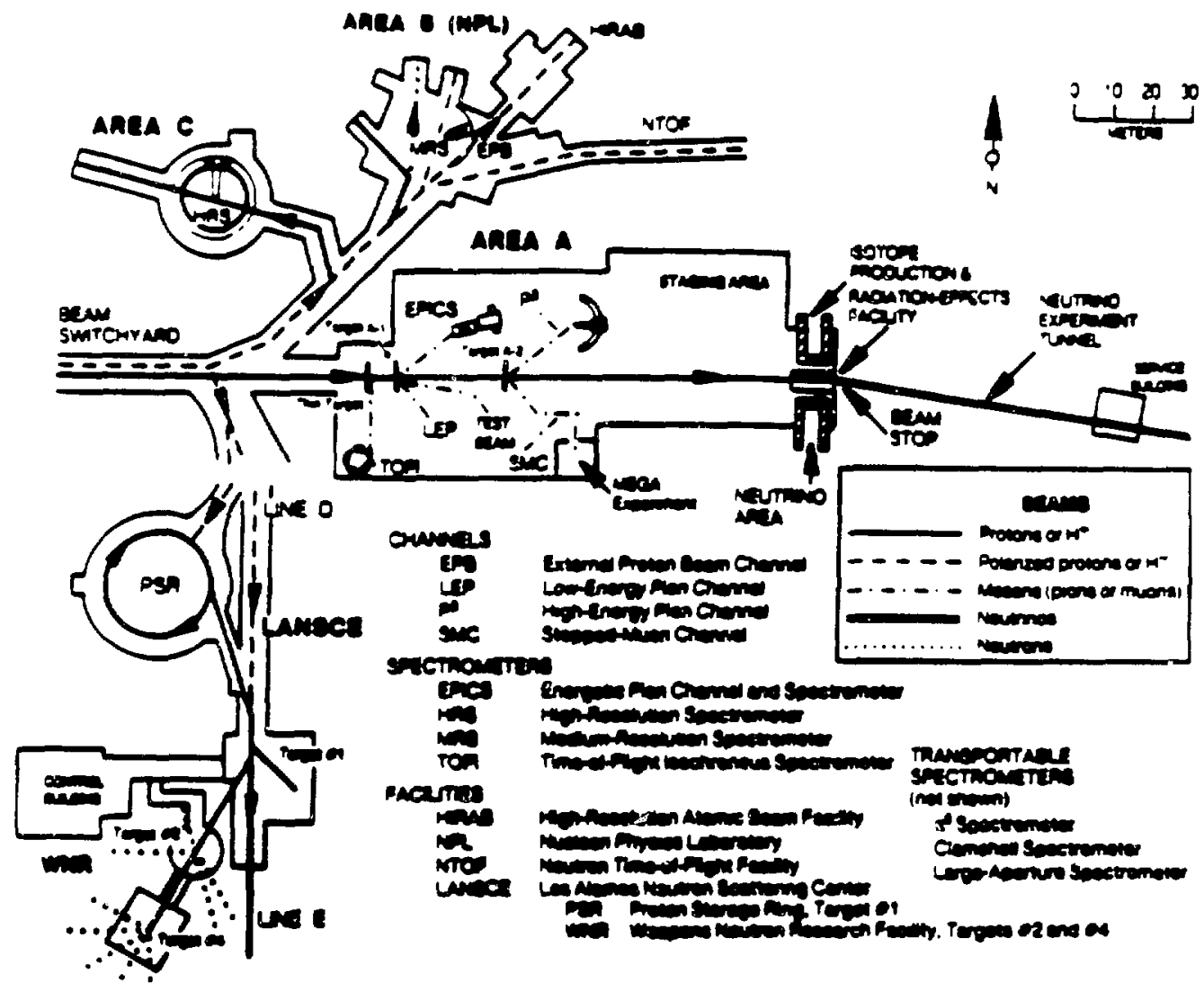

Figure I-1.

Exparimental areas at LAMPP. 


\section{II-2. EPICS channel}

The EPICS channel gets its beam from the A-1 target, a rotating graphite wheel. The channel consists of four dipole bending magnets (BM01-4), four collimator sets known as jaws (F J01-4), and three multipole focusing m? znets (FM013), as shown in Figure II-2. The channel's optics, also shown in Figure II-2, are point to point focusing in the vertical direction and point to parallel in the horizontal direction. Some properties of the pion beam are given in Table II-1.

In order to provide the maximum flux of pions without sacrificing momentum resolution a dispersed beam is used. The particle's position in the dispersion direction (vertical for EPICS) in the focal plane, the position of the target, is correlated with its momentum. The range of momentum from the channel is given by

$$
\delta_{c}=\frac{P_{\pi}-P_{e}}{P_{c}} \times 100 \%,
$$

where $P_{c}$ is the central momentum of the channel determined by the fields in the dipoles. The dispersion of the channel is $\approx 10 \mathrm{~cm} / \%$. When the jaws are wide open the channel provides a beam with $\delta_{e}= \pm 1 \%$, giving a beam which is $20 \mathrm{~cm}$ high.

The EPICS beam is monitored in three places. The first is a toroid around the main proton beam before the A-1 target, 1ACM02; the second is a scintillator at the A-1 target, BOT, and finally with an ion chamber in the scattering chamber, IC1. For extreme forward angles, $\theta<20^{\circ}$, the ion chamber blocks the spectrometer and cannot be used. The EPICS beam is not pure pions. A large variety of particles and nuclei are produced at the A-1 target. A thin sheet of Mylar separating the channel from the scattering chamber and the spectrometer functions as a gas barrier to keep radioactive gases such as ${ }^{\circ} \mathrm{He}$ and ${ }^{8} \mathrm{He}$, produced in $\mathrm{p}^{12} \mathrm{C}$ collisions at the A-1 target, from entering the detection system. Muons and protons are also 


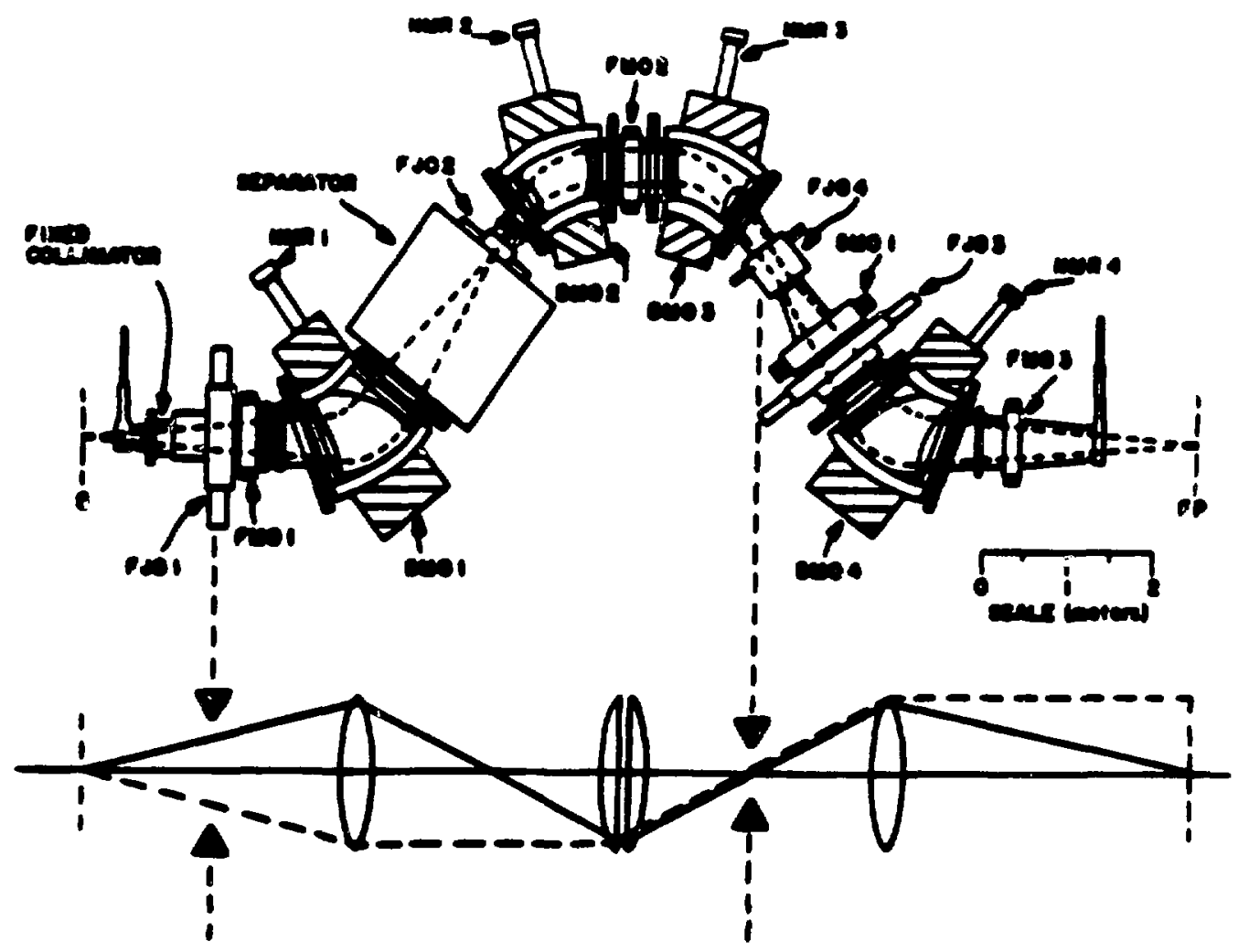

Firure [1-2.

Schomatic of the EPICS channel. 
TABLE II-1.

EPICS beam spot characteristics.

Width

Height

Horizontal divergence

Vertical divergence

Momentum dispersion

Momentum resolution $\pm 4 \mathrm{~cm}$ ( $Y$ coordinate)

$\pm 10 \mathrm{~cm}$ ( $\mathrm{X}$ coordinate)

$\pm 15 \mathrm{mr}$ ( 9 coordinate)

$\pm 77 \mathrm{mr}$ ( $\phi$ coordinate)

$0.1 \% / \mathrm{cm}$ in $\mathrm{X}$

$2.0 \times 10^{-4} \mathrm{FWHM} \Delta \mathrm{p} / \mathrm{p}$

TABLE II-2.

Isotopic composition of ${ }^{20} \mathrm{Ne}$ gas.

\begin{tabular}{cc}
\hline Isotope & Mole fraction \\
\hline Neon-20 & 99.97 Mole \% \\
Neon-21 & 0.03 Mole \% \\
\hline Gross composition of ${ }^{20}$ Ne gas. \\
\hline Chemical & Mole fraction \\
\hline Neon & 99.94 Mole \% \\
$\mathrm{H}_{2}$ & $<0.1$ Mole \% \\
$\mathrm{H}_{2} \mathrm{O}$ & $<0.1$ Mole \%
\end{tabular}


in the beam. A proton degrader can be put in between BM03 and BM04. Protons loge more energy in the degrader than pions, so they have a lower momentum in BM04 than pions and therefore are not bent into the scattering chamber. Unfortunately this also degrades the resolution of the pion beam, so a high resolution experiment like the present one cannot use it. Because pions decay into muons there are also muons in the beam. Since muons have approximately the same mass as pions, $M_{\mu}=106 \mathrm{MeV}$ and $M_{\pi}=140 \mathrm{MeV}$, it is not possible to separate them by their momentum. Muon rejection will be discussed later in this chapter.

\section{II-3. EPICS spectrometer}

The EPICS spectrometer consists of three quadrupole focusing magnets (QM01-3), two dipole bending magnets (BM05-6), 3 gets of wire chambers, 8 or 9 scintillators, and a Cherenkov detector as shown in Figure II-3. The quadrupoles are used to focus an image of the target on the front set of wire chambers. This tells the position on the target of the pion, and, because the dispersion of the beam is known, the incident energy of the pion. The dipole magnets each provide a $60^{\circ}$ bend to measure the momentum of the scattered particle. The scattered momentum is measured with respect to the central momentum of the spectrometer

$$
\delta_{\mathrm{sp}}=\frac{\mathrm{P}_{\pi}-\mathrm{P}_{\mathrm{sp}}}{\mathrm{P}_{\mathrm{sp}}} \times 100 \%
$$

where $P_{s p}$ is the central momentum of the spectrometer. The dipole magnets focus the particles on the rear wire chambers. The useful acceptance of the spectrometer is approximately $\pm 6 \%$.

The Cherenkov and scintillators are mainly used for particle identification. The Cherenkov and S1, the scintillator located between the quadrupoles and the front chambers, were not used in this experiment. Electrons are the only particles 


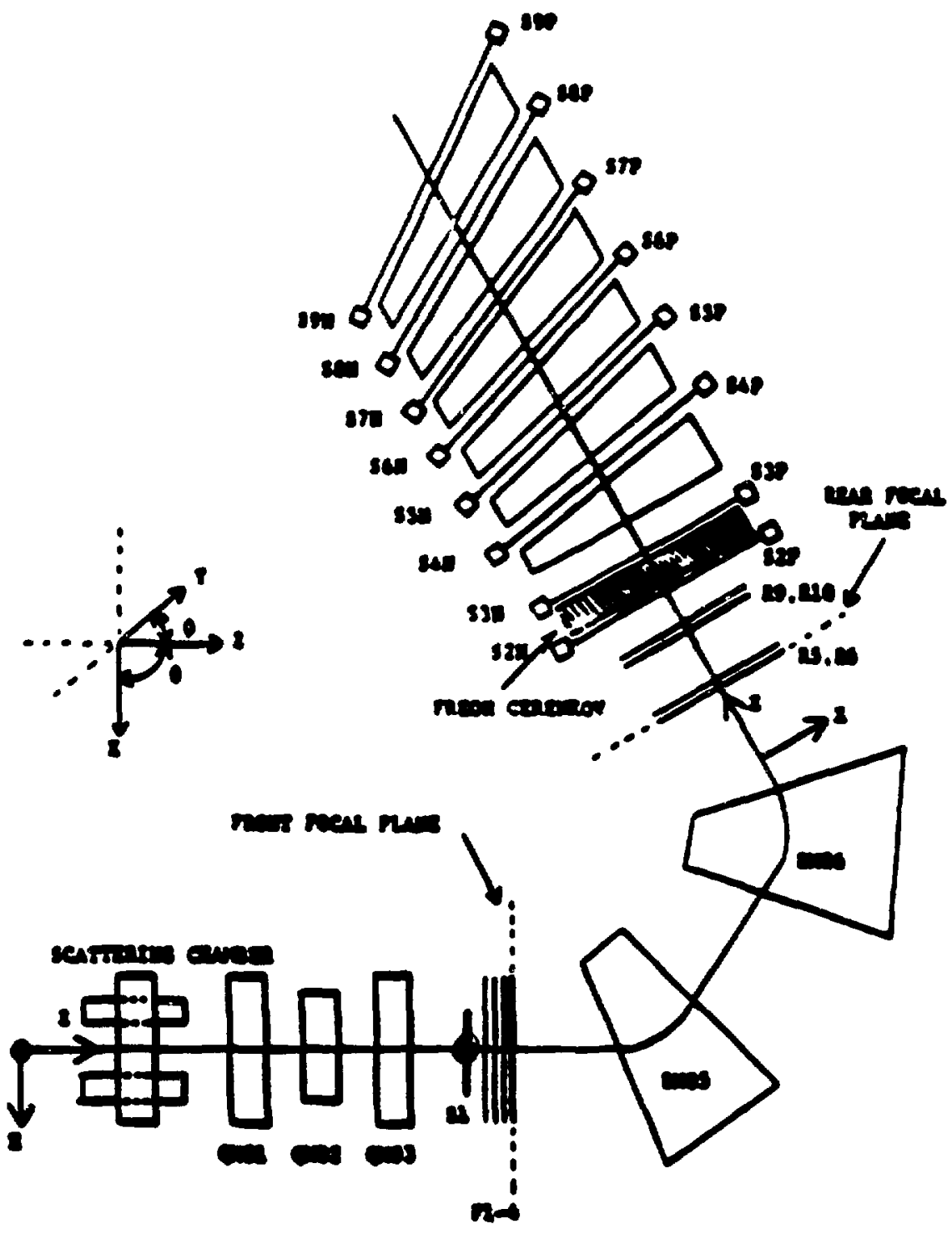

Figure [1-3.

Schematic of the EPICS spectrometer. 
relativistic enough for the Cherenkov to detect, and they are a problem only in double charge exchange (DCX) reactions. S1, located before the dipoles, was not used because it degrades the energy regolution. The scintillators $S 4$ through $S 9$ are used in muon rejection. They are separated by graphite blocks and the pions are ranged out in the blocks. The procedure is fine tuned by placing aluminum slabs between S3 and S4. A two-dimensional plot of the time of flight between S2 and $\mathrm{S} 3$ versus the mean pulse height in $\mathrm{S} 2$ and $\mathrm{S} 3$ is used to reject protons. $\mathrm{S} 2$ is also used to define the timing for the wire chambers.

The wire chambers are used to define the position of the pion in the front and rear focal planes. They also measure the angle of the pion's trajectory relative to a central ray passing through the spectrometer. This information is used to calculate the position on the target the pion came from, the angle it left the target with, and the energy of the pion before and after its interaction with the target. This will be described in greater detail in section $E$.

A schematic diagram of the electronics is shown in Figure II-4. A good event is defined as any event that triggers $S 2$ and $S 3$ and any one of the front chambers. Using normal CAMAC electronics, it was possible to get only one event per beam gate. In the spring before this experiment ran LeCroy FERA electronics were installed. Under this system the ADC and TDC values are stored in FERA's memory and are read out to the MBD either when the memory is full or at the end of the beam gate. This enables the acquisition of up to 10 or 11 events per beam gate. Our data acquisition rate was limited by the front set of wire chambers. At times when there was a very large counting rate, it was necessary to close the jaws, collimators, to prevent damage to the front wire chambers. The current experiment never averaged more than 4 events/beam gate at any given getting and generally had fewer than 1 event/beam gate. 


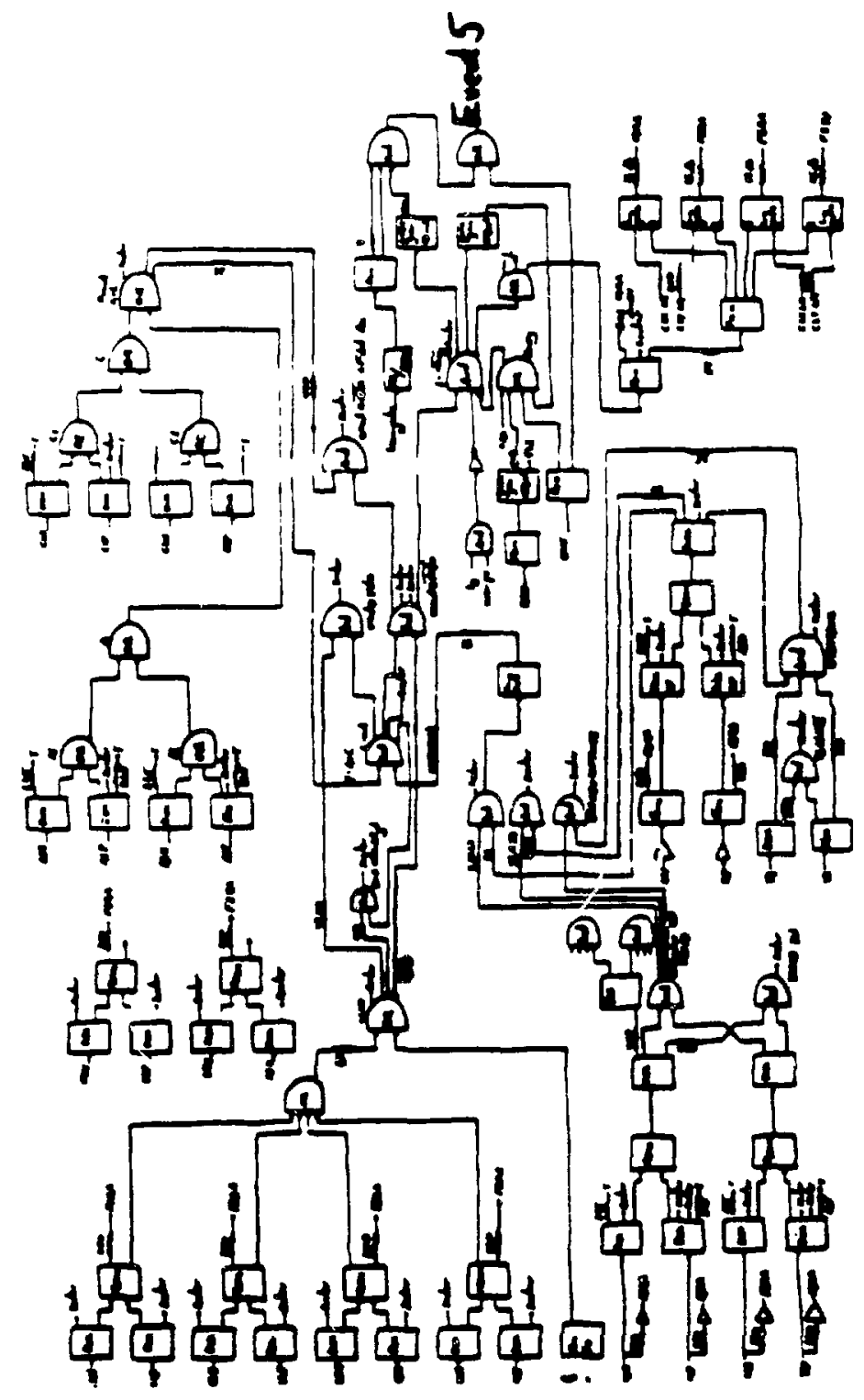

Figure III-4.

Schematic of the EPICS electronics. 


\section{II-4. EPICS cooled gas target}

Isotopically enriched ${ }^{20} \mathrm{Ne}$ gas was used in the EPICS cooled gas cell and the rare gas handling system. The target's composition is given in Table II-2. A diagram of the gas cell is shown in Figure Il-5. The gas pressure in the cell was $\approx 20$ psi. The neon was cooled to $\approx 45 \mathrm{~K}$ using liquid helium. This gave an effective target thickness of $=100 \mathrm{mg} / \mathrm{cm}^{2}$. The temperature of the gas was monitored at Gve places in the gas cell, labeled T1-T5 in Figure II-5. The temperature and pressure of the gas were recorded every 2-3 hours, and were very stable throughout the experiment. Normalizations were done by pumping the seon out of the cell, putting hydrogen gas in, and measuring $\pi p$ elastic scattering.

\section{II-5. Analyois of events}

The data were acquired and replayed using the $Q$ system, the standard data acquisition and replay programs used at LAMPF, and MP-10 software written to work with the $Q$ software and to handle the more specialized requirements of EPICS. When the beam turns on, before any experiment can run, certain calibrations must be done. As mentioned above, the analyzer calculates where on the target the pion came from, and the energy of the pion before and after the interaction.

Consider $x_{\text {trt }}$, parameter 233 , the position on the target in the vertical direction, as an example. The analyzer reads in a calibration from a file POL.DAT, shown in Table II-3. Using this calibration it calculates $\mathbf{x}_{\mathbf{t} \text { t }}$ :

$$
\begin{gathered}
\mathrm{x}_{\mathrm{tgt}}=0.157582-0.991615 \times \mathrm{x}_{\text {front }}-0.499238 \times 10^{-2} \times \theta_{\text {front }} \\
-0.195730 \times 10^{-2} \times \mathrm{x}_{\text {front }} \times \delta_{\text {crude }}-0.330807 \times 10^{-2} \times \theta_{\text {front }} \times \delta_{\text {erude }}+\cdots
\end{gathered}
$$

where the other parameters are either the raw data or calculated from the raw 
- 25 .

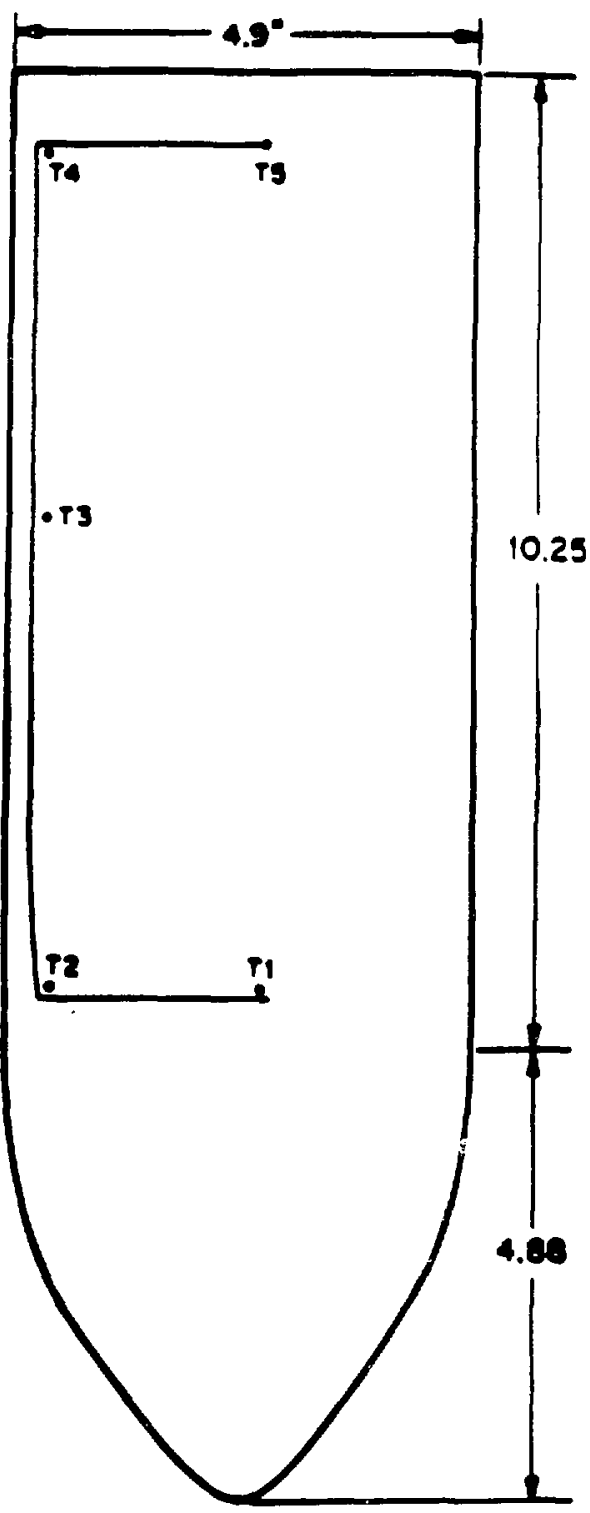

Figure II-5.

Diagram of the EPICS cooled cae cell. T1-T5 indicate the ponitions of the five temperature senvors. 


$$
-26-
$$

TABLE II-3.

$\mathbf{x}_{\text {tgt }}$ polynomial form POL.DAT.

$\begin{array}{cc}\text { Data word } & \text { Coefficient } \\ 0,0,0,0 & 0.157582 \mathrm{E}+00 \\ 221,0,0,0 & -0.991615 \mathrm{E}+00 \\ 222,0,0,0 & -0.499238 \mathrm{E}-02 \\ 221,243,0,0 & -0.195730 \mathrm{E}-02 \\ 222,243,0,0 & -0.330807 \mathrm{E}-02 \\ 222,223,0,0 & 0.133496 \mathrm{E}-04 \\ 222,224,0,0 & -0.295376 \mathrm{E}-04 \\ 221,243,243,0 & 0.325431 \mathrm{E}-03 \\ 222,243,243,0 & -0.459714 \mathrm{E}-04 \\ 221,223,223,0 & -0.391464 \mathrm{E}-03 \\ 221,223,224,0 & 0.555083 \mathrm{E}-05 \\ 221,224,224,0 & 0.128760 \mathrm{E}-04 \\ 221,221,221,0 & 0.191659 \mathrm{E}-04 \\ 221,221,222,0 & 0.759415 \mathrm{E}-04 \\ 222,222,222,0 & 0.499401 \mathrm{E}-06 \\ 222,223,223,0 & 0.340980 \mathrm{E}-03 \\ 222,223,224,0 & -0.118167 \mathrm{E}-03 \\ 222,224,224,0 & 0.106321 \mathrm{E}-04 \\ 243,0,0,0 & 0.113247 \mathrm{E}-01\end{array}$

1.) Where data word 221 is $x_{\text {front }}, 222$ is $\theta_{\text {front }}, 223$ is $y_{\text {front }}, 224$ is $\phi_{\text {front }}$, and 243 is $\delta_{\text {crude }}$. 
data at some earlier point by the analyzer. Before any experiment can run these polynomials must be calibrated. For $x_{\text {tgt }}$ this is done by putting in a target with five horizontal rods at $x= \pm 8 \mathrm{~cm}, x= \pm 4 \mathrm{~cm}$, and $x=0 \mathrm{~cm}$. A sample $x_{\text {tgt }}$ spectrum with the rods is shown in Figure II-6. Data are taken with this target and the data words used in the polynomial are written to disk for each good event. Then the coefficients in the polynomial are varied to give the best fit to the known positions of the rods. These best fit coefficients are written to POL.DAT. It is particularly important to know $x_{\text {tgt }}$ well, because the beam is dispersed in the vertical direction. Table II-4 lists several other quantities calibrated in a similar manner.

Except for $\theta_{\text {chk }}, \phi_{c h k}$, and $\delta$, all the calibrations must be done with flat, solid targets. The efficiency of the chambers is measured with $\theta_{\text {chk }}$ and $\phi_{\text {cbl }}$. Because the gas cell is an extended target this can lead to errors in calculating these quantities, as shown in Figure II-7. The diameter of the gas cell is 4.90 in., go if the interaction occurred at the edge of the gas cell, the correct value of $x_{t g}$ is undetermined by $2 \delta x$. The vertical divergence of pions entering the spectrometer is \pm 50 mrad, $\phi$ in Figure II-7, which gives a $\delta x$ of $0.31 \mathrm{~cm}$. Since the pion beam is dispersed in the $\mathbf{x}$ direction this gives an uncertainty of approximately $80 \mathrm{keV}$ in the incident energy of the pion and therefore in the calculation of the missing mass spectrum, which worsens the resolution. Finally, the energy of the outgoing pion is determined by $\delta$, therefore it is necessary to know $\delta$ as well as possible, so data taken on neon with the gas cell were used to calibrate $\delta$.

\section{II-6. Normalizations}

Absolute normalizations were obtained by putting $\mathrm{H}_{2}$ in the gas cell, measuring $\pi$-p scaitering and comparing the yields to cross sections calculated from the $\pi$-nucleon phase shifts of Rowe, Salomon, and Landau (RO-78). Angular 


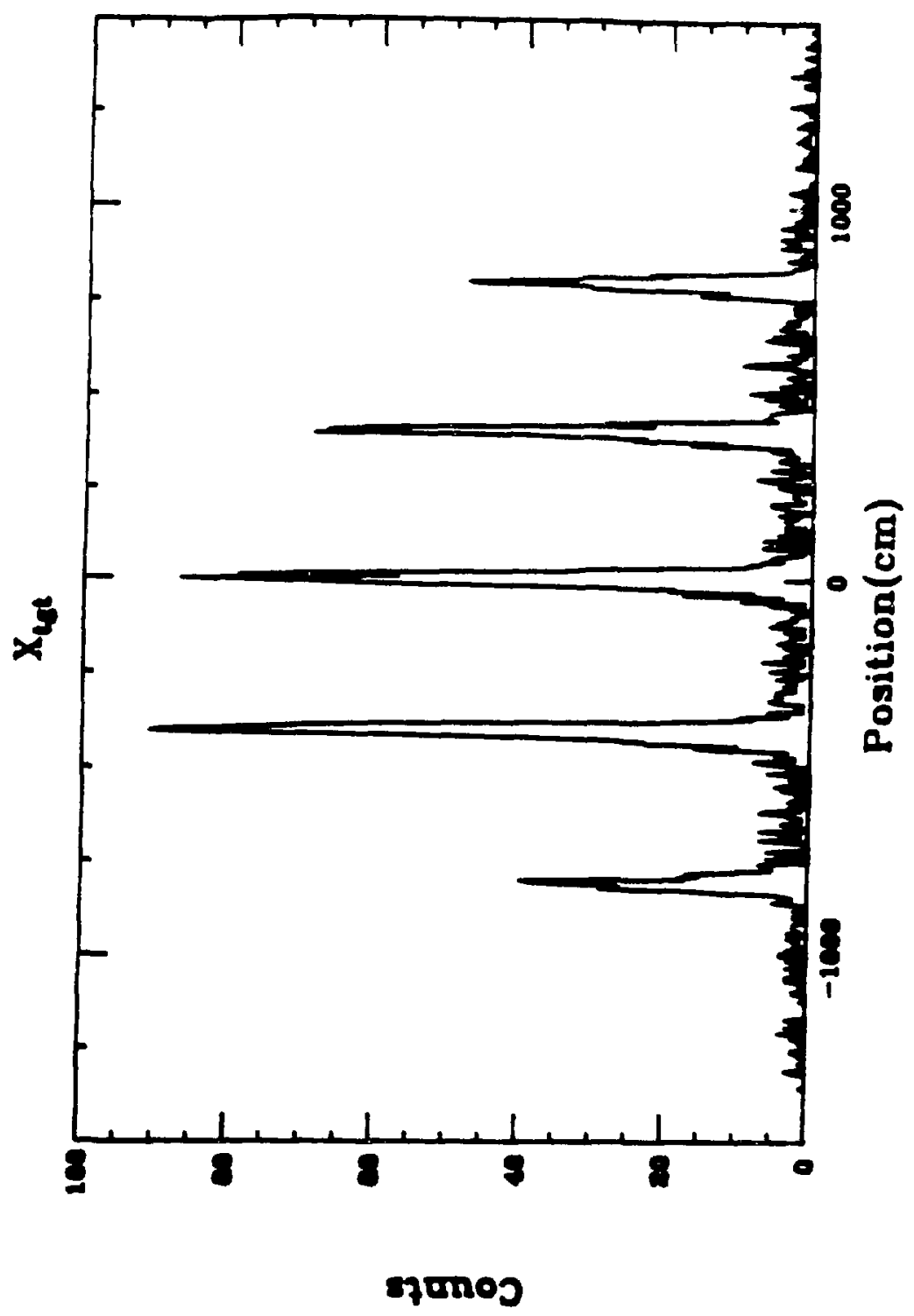

Figure II-6.

4. spectrum with horisontal rod target. 
TABLE II-4.

$\begin{array}{cl}\text { Parameter } & \text { Some quantities calculated from POL.DAT. } \\ x_{\text {tgt }} & \text { vertical position on target } \\ y_{\text {tgt }} & \text { horizontal position on target } \\ \theta_{\text {tgt }} & \text { angle between the outgoing pion's trajectory } \\ & \text { and the } 2 \text {-axis }{ }^{1} \text { in the } x-z \text { plane } \\ \phi_{\text {tgt }} & \text { angle between the outgoing pion's trajectory } \\ & \text { and the } 2 \text {-axis }{ }^{1} \text { in the } y-2 \text { plane } \\ \theta_{\text {ebk }} & \text { difference between } \theta \text { in the front chambers } \\ & \text { and } \theta \text { in the rear chambers } \\ \phi_{\text {chk }} & \text { difference between } \phi \text { in the front chambers } \\ & \text { and } \phi \text { in the rear chambers } \\ \delta & \delta=100 \times\left(P_{\pi}-P_{s p}\right) / P_{s p} \\ & \text { where } P_{s p} \text { is the central momentum of the spectrometer } \\ & \text { and } P_{\pi} \text { is the momentum of the outgoing pion. }\end{array}$

1.) The $z$-axis is defined to be the beam axis with positive $z$ in the beam direction.

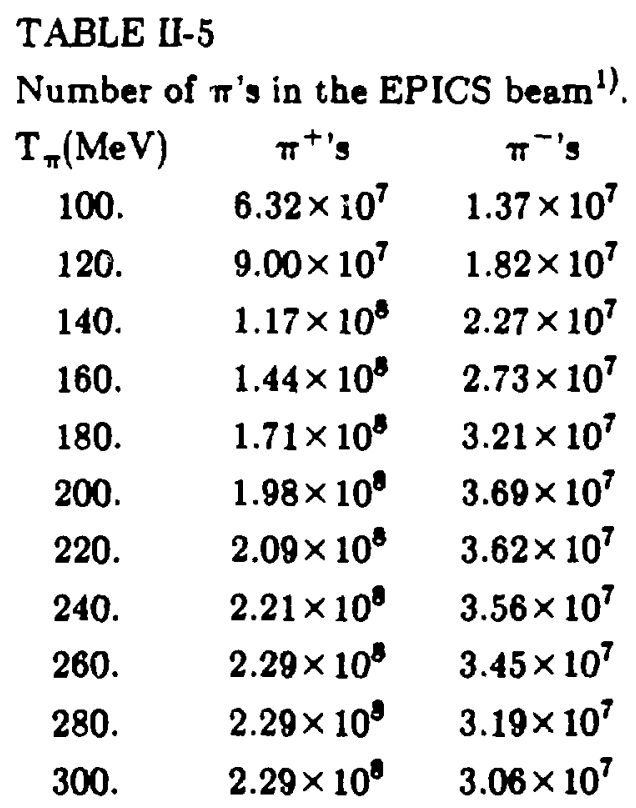

1.) With a $1 \mathrm{~mA}$ proton beam and the jaws open. 


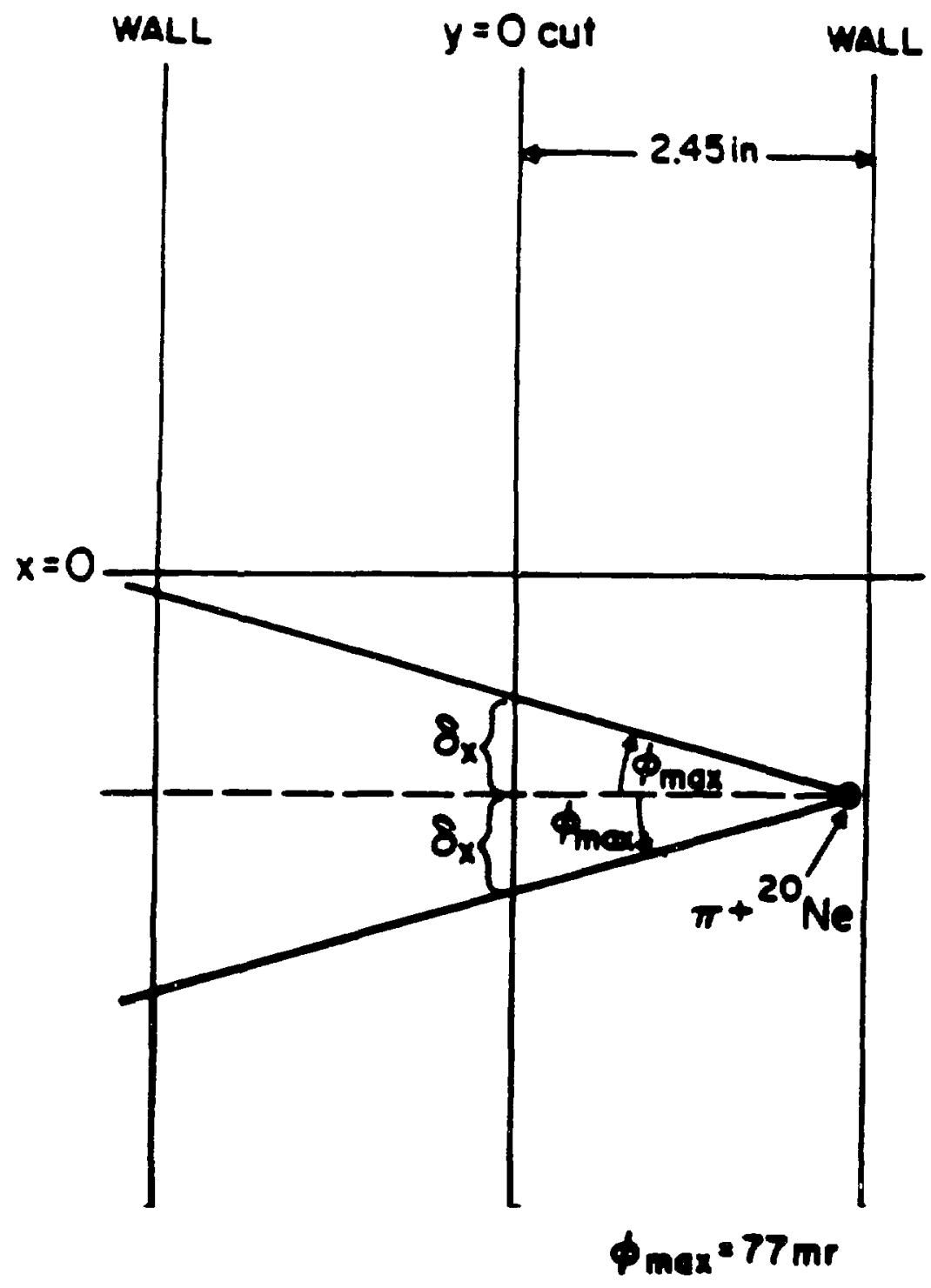

Figure II-7.

View of the gas cell alons $y=0$. The radius of the cas cell is 2.45 in. As described in the text, $8 x$ in the uncertainily in calculation of $x_{\text {ip. }}$. 
distributions calculated with these phase shifts are shown in Figure II-8. Because, as shown in Figure II-9, a different volume of the gas cell is sampled as the spectrometer angle is changed, a ${ }^{1} \mathrm{H}\left(\pi^{+}, \pi^{+}\right)^{1} \mathrm{H}$ angular distribution was taken at $T_{\pi}=180 \mathrm{MeV}$. Figure II-10 shows the angular dependence determined by normalizing the hydrogen yields to the calculated cross sections. Because the number of pions in the beam depends on the energy of the beam, as shown in Table II-5, hydrogen data were also taken for $180-\mathrm{MeV}^{-}$and $120-\mathrm{MeV}^{+}$.

The effective solid angle of the spectrometer also depends on the momentum of the outgoing pion or $\delta$. This dependence can be measured by varying the central momentum of the spectrometer and measuring the yield of a peak at each setting of $\delta$, a procedure known as an acceptance scan. This dependence is show in Figure II-11.

In the current experiment the statistical error on the relative normalizations is better than $2 \%$ except at the extremes of the acceptance scan where it is $2.5 \%$. The absolute errors are primarily uncertainties in the ratio of the $\mathrm{H}_{2}$ to the Ne target thickness, $\approx 3 \%$, and the $\pi$-nucleon cross section, $\approx 10 \%$. Therefore the total relative normalizations should be accurate to better than $4 \%$ and the absolute normalizations to better than $11 \%$. 


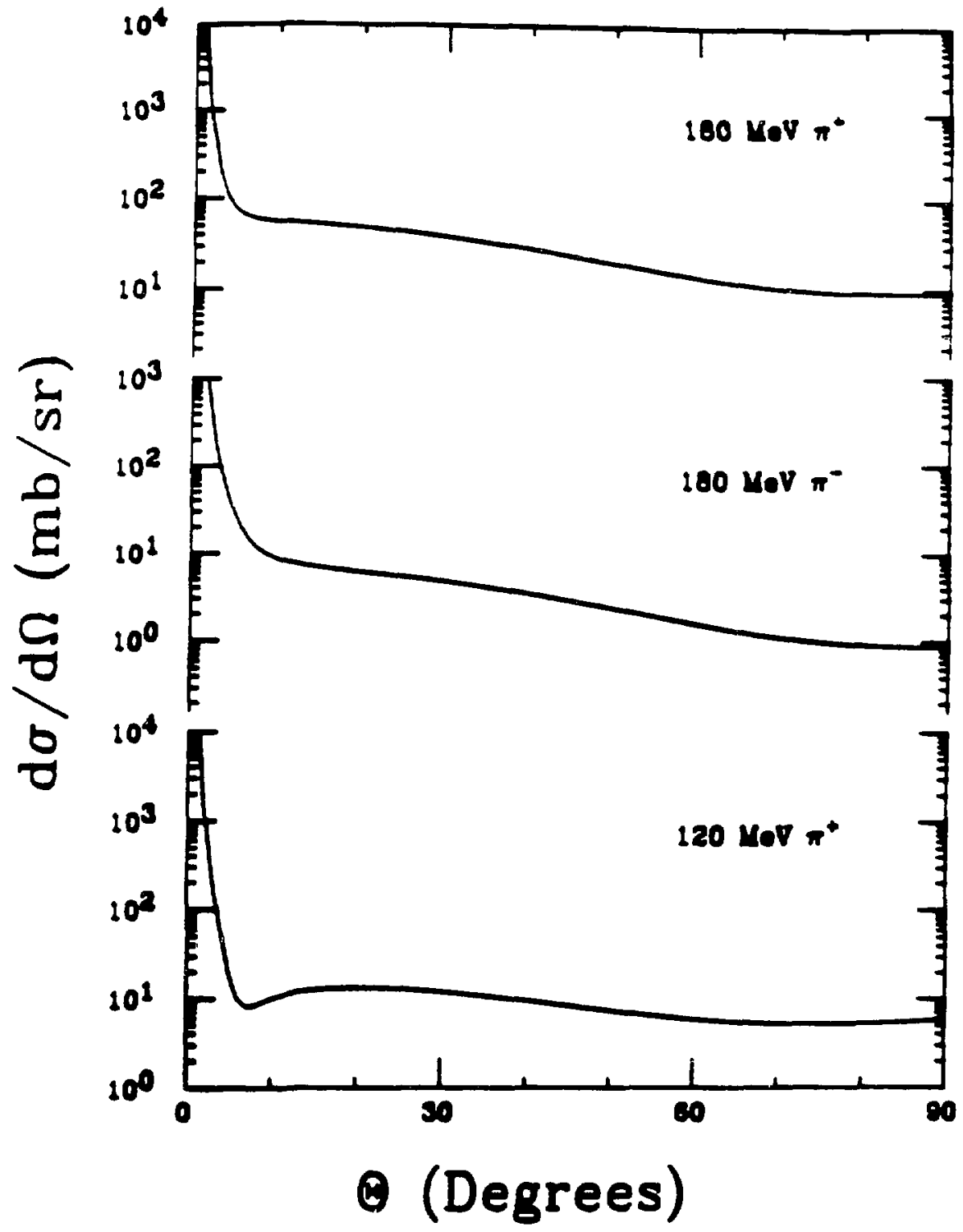

Firure 10 -8.

${ }^{1} H(\pi, \pi)^{1} H$ angulas distributions calculated wing the phase shifte of Rowe, Salomon, and Landeu (RO-78). 
(a)

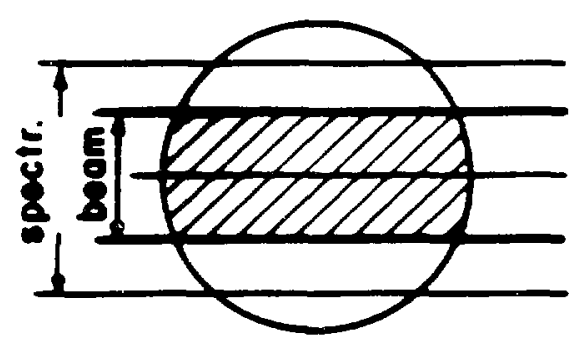

(c)

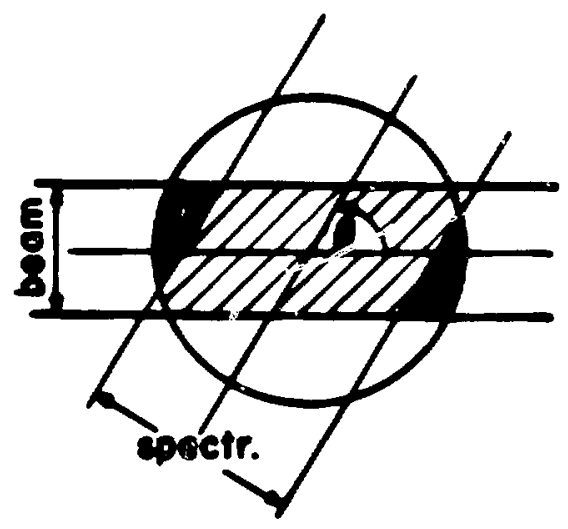

(b)

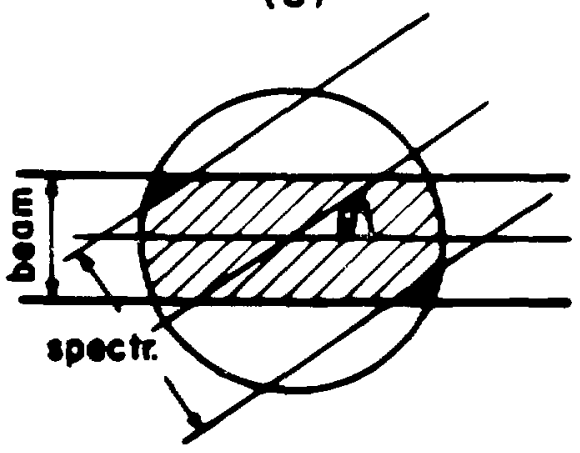

(d)

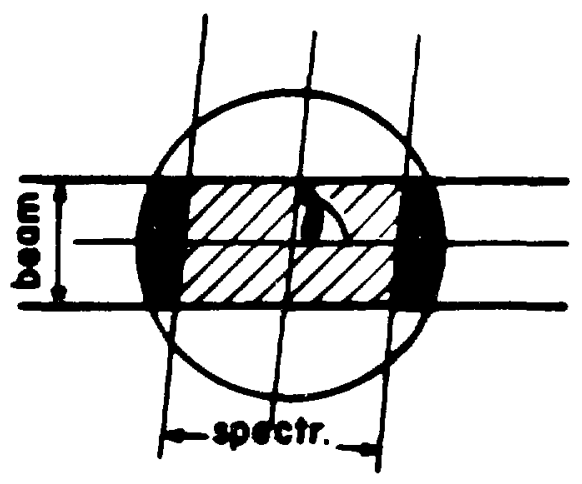

Pipare I-9.

Sehematic of the sections of the target and gas cell the spectrometer son a a fuaction of angle. The aheded action is gas which in son by both the spactrometer and the beam. The solid section in gen which is seen by the beam, but not the spectrometar. For small $\theta$ the opectrometer soes all of the target and gas cell that the pion beam an, an is shown in saction a. As the spectrometar is mored to larges angles some of the target and gas cell are no longer sean, a shown in saction b. As it is mored to still larger anglen lew gas is seen by both the beam and the spectrometer, and it becomen poevible to exparate the front and rear wall of the sas cell a shown is section c. Ereritislly the spectrometer reachow a point where it ao longer sees the wall of the gen cell so shown in section d. The dependence on $\theta$ is obviously symmetric around 900 . 
Relative Solid Angle
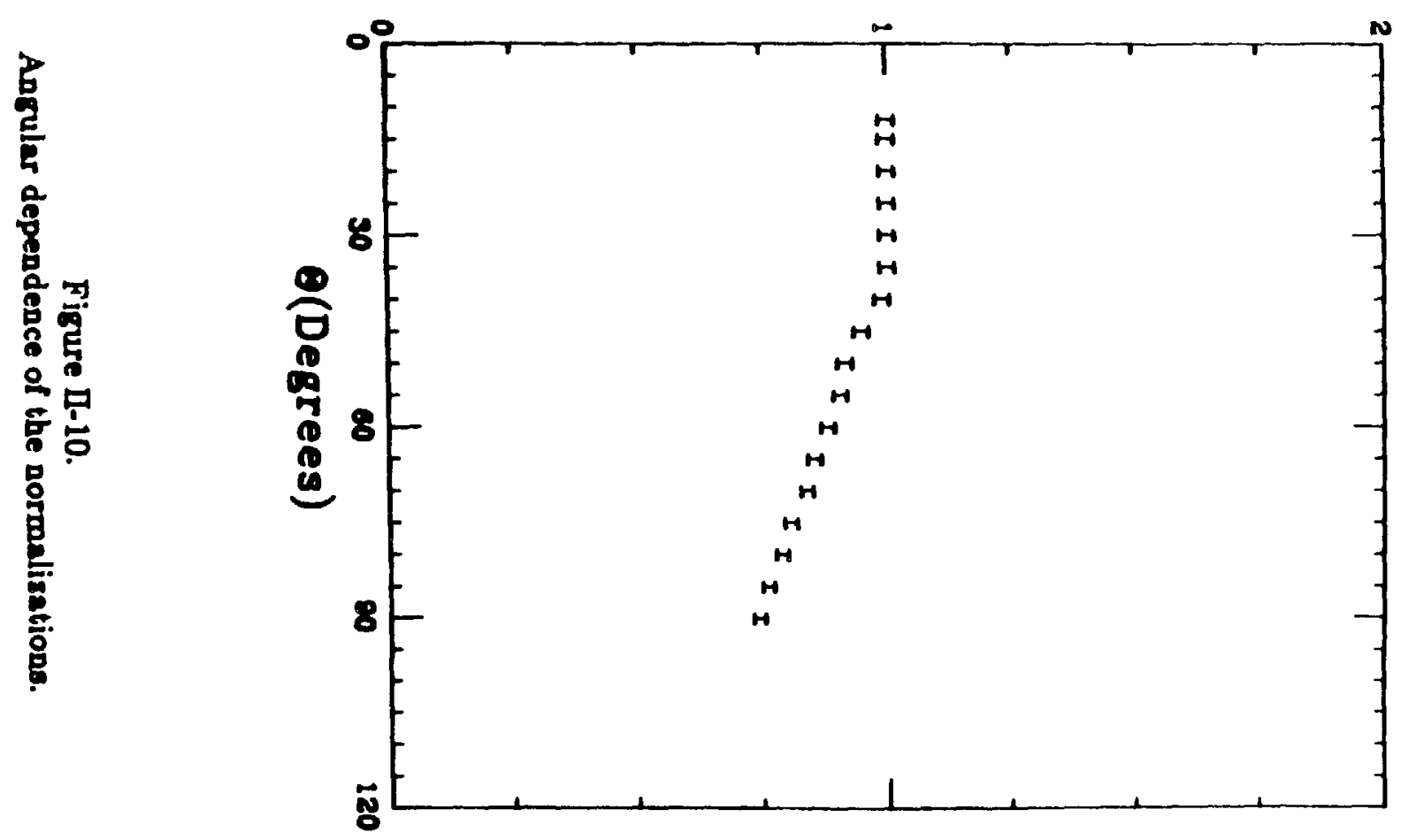
Relative Acceptance

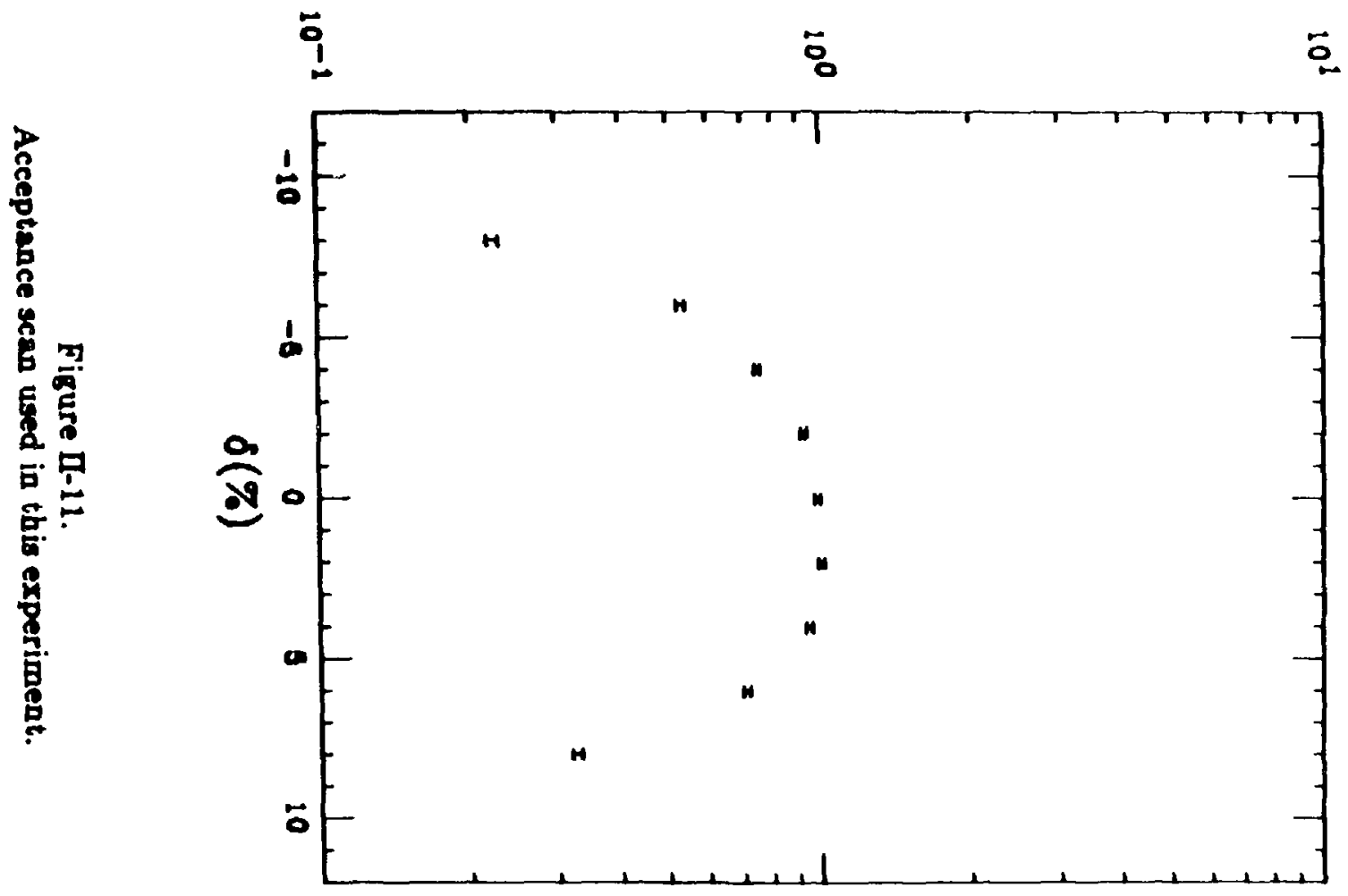

$\dot{1}$ 


\section{Nuclear Structure of ${ }^{20} \mathrm{Ne}$}

Nuclear theory can be roughly divided into two parts: reactions and structure. Reactions which are well understood can be used to study the structure of states in nuclei which are not well understood and similarly well-understood states in nuclei can be used to study reaction mechanisms. The basic structure of many low-lying states in ${ }^{20} \mathrm{Ne}$ is well known. They can be understood as sotations of a very deformed nucleus, as will be described in the first section of this chapter. They can also be explained by spherical shell-model calculations, as described in the second section. Both of these descriptions will be used in the analysis of the data presented bere. A useful qualitative understanding of their structure comes from the Nilsson model which will be explained in the third section of this chapter.

\section{III-1. Collective Model}

The simpleat collective theories of the nucleus model it as a liquid drop. This gection will follow the derivation of Preston and Bhaduri (PR-75). For even-even nuclei the spins of the individual nucleons will pair to zero for the low-lying states, so these states may be taken as arising from the rotational and vibrational modes 
of a deformed liquid drop. Therefore the intringic motion of the nucleons will be ignored.

For permanently deformed nuclei, i.e. nuclei with a non-spherical shape whose shape does not change with time, it is convenient to define the nuclear surface with espect to the principal axes of the nucleus and to specify the orientation of those body-fixed axes with respect to space-fixed axes with time-dependent Euler angles $\left(\theta_{1}, \theta_{2}, \theta_{3}\right)$. The nuclear surface can be described as:

$$
R=R_{0}\left[1+\sum_{\lambda=2 \mu=-\lambda}^{x} \sum_{\lambda, \mu}^{\lambda} Y_{\lambda}^{\mu}(\theta, \phi)\right]
$$

For simplicity only quadrupole $(\lambda=2)$ shapes will be considered. Then $a_{2,1}=a_{2,-1}=0$ and $a_{2,2}=a_{2,-2}$ since the principal axes are the body-fixed frame. A convenient set of variables often used is

$$
\mathrm{a}_{2,0}=\beta \cos \gamma
$$

and

$$
a_{2,2}=\frac{1}{\sqrt{2}} \beta \sin \gamma
$$

Since

$$
\beta^{2}=\sum_{\mu} a_{2 \mu}^{2}
$$

$\beta$ is obviously a measure of the total deformation. The quantity $\gamma$ is a measure of the type of deformation, which can be seen by looking at $R(\theta, \phi)$ along each axis. By substituting the expressions for $Y_{2}^{\mu}, a_{2,0}$, and $a_{2,2}$ the equation for the nuclear surface becomes:

$$
R-R_{0}=\left(\frac{5}{16 \pi}\right)^{3} R_{0} \beta\left[\cos \gamma\left(3 \cos ^{2} \theta-1\right)+\sqrt{3} \sin \gamma \sin ^{2} \theta \cos 2 \phi\right] .
$$


Evaluating this expression on the $x, y$, and $z$ body axes gives:

$$
\begin{aligned}
& \delta R_{x}=\left(\frac{5}{4 \pi}\right)^{\frac{4}{2}} R_{0} \beta \cos \left(\gamma-\frac{2 \pi}{3}\right) \\
& \delta R_{y}=\left(\frac{5}{4 \pi}\right)^{1 / 3} R_{0} \beta \cos \left(\gamma-\frac{4 \pi}{3}\right)
\end{aligned}
$$

and

$$
\delta R_{2}=\left(\frac{5}{4 \pi}\right)^{4} R_{0} \beta \cos \gamma .
$$

If we write the axes $x, y, z$ as $1,2,3$ then these three expressions become for $k=1,2,3$

$$
\delta R_{\kappa}=\left(\frac{5}{4 \pi}\right)^{4} \beta R_{0} \cos \left(\gamma-\frac{\kappa 2 \pi}{3}\right)
$$

From these equations it is obvious that $\gamma, \gamma-2 \pi / 3$, and $\gamma-4 \pi / 3$ all describe the same surface with the axes permuted. Therefore only values of $\gamma$ from 0 to $\pi / 3$ are needed. Also from these equations one can see that a nucleus with $\gamma=0$ is a prolate ellipsoid and $\gamma=\pi / 3$ is an oblate ellipsoid.

In order to extract physical quantities such as excitation energies and transition probabilities, a Hamiltonian and wave functions are needed. For a deformed liquid drop it can be shown (for example PR-75) that the kinetic energy, $T$, can be written as

$$
T=1 / 2 B\left(\beta^{2}+\beta^{2} \dot{\gamma}^{2}\right)+1 / 2 \sum_{k=1}^{3} I_{k} \omega_{k}^{2},
$$

where the first term is the vibrational kinetic energy, $T_{\beta}+T_{\gamma}$, and the second is the rotational kinetic energy. In this equation $\omega$ is the angular velocity of the principal (body-fixed) axes with respect to space-fixed axes and $I_{k}$ is the effective moment of inertia given by 


$$
I_{k}=4 B \beta^{2} \sin ^{2}\left(\gamma-\frac{k 2 \pi}{3}\right) \text {. }
$$

The Hamiltonian then takes the form

$$
H=T_{\beta}+T_{\gamma}+\sum_{\kappa=1}^{3} \frac{L_{k}^{2}}{2 I^{k}}+V(\beta, \gamma)
$$

For a stiff, deformed nucleus the potential $V(\beta, \gamma)$ will have a narrow, deep minimum. In addition to rotation, the nucleus can vibrate about the minimum. Expanding $V(\beta, \gamma)$ and $I_{\kappa}$ around the minimum, $\left(\beta_{0}, \gamma_{0}\right)$, the Hamiltonian becomes

$$
\begin{gathered}
H=T_{\beta}+4 / 2 C_{\beta}\left(\beta-\beta_{0}\right)^{2}+T_{\gamma}+4 C_{\gamma}\left(\gamma-\gamma_{0}\right)^{2} \\
+\sum_{k=1}^{3} \frac{L_{k}^{2}}{2 I_{k}\left(\beta_{0}, \gamma_{0}\right)}+U_{1}+U_{2}
\end{gathered}
$$

where $C_{\beta, \gamma}$ come from the expansion of the potential, $V(\beta, \gamma)$, and $U_{1}$ and $U_{2}$ are the rotation-vibration interaction. To first order in $\beta-\beta_{0}$ and $\gamma$

$$
U_{1}=-\frac{L^{2}}{I_{0}}\left(L^{2}-L_{3}^{2}\right) \frac{\beta-\beta_{0}}{\beta_{0}}
$$

and

$$
\mathrm{U}_{2}=-\frac{\mathrm{U}^{2}}{2 \mathrm{~L}_{0}}\left(\mathrm{~L}_{+}^{2}+\mathrm{L}_{-}^{2}\right) \frac{\gamma}{\sqrt{3}},
$$

where $\mathrm{L}_{+}$and $\mathrm{L}_{-}$are the raising and lowering operators for angular momentum. $U_{1}$ and $U_{2}$ can be treated in pertubation theory. This Hamiltonian is known as the Bohr Hamiltonian.

The Hamiltonian is separable into rotational and vibrational parts if $U_{1}$ and $U_{2}$ are neglected. If $I_{1}=I_{2}$ then the projection of the angular momentum along the third axis, $K$, is a good quantum number. For unequal $I_{\kappa}$ the wave function can be 
written as

$$
\Psi=f_{\mathrm{J}, \mathrm{T}, \nu}(\beta) \sum_{\mathrm{K}=0}^{\mathrm{J}} g_{K, \mathrm{~J}, \mathrm{\tau}} \mid \mathrm{JMK}>
$$

where

$$
\mid \mathrm{JMK}>=\left[\frac{2 \mathrm{~J}+1}{16 \pi^{2}\left(1+\delta_{\mathrm{k} 0}\right)}\right]^{\mathrm{h}}\left(\mathrm{D}_{\mathrm{M}, \mathrm{K}}^{\mathrm{J}}\left(\theta_{1}, \theta_{2}, \theta_{3}\right)+(-)^{\mathrm{J}} \mathrm{D}_{\mathrm{M},-K}^{J}\left(\theta_{1}, \theta_{2}, \theta_{3}\right)\right) .
$$

The $D_{M, K}^{J}$ are simultaneous eigenfunctions of $J^{2}, J_{3}$, and $J_{2}$, where $J_{3}$ is the component of the angular momentum along the 3-axis in the body-fixed system, and $\mathrm{J}_{2}$ is the component of the angular momentum along the z-axis of the space-fixed system such that

$$
\begin{gathered}
\mathrm{J}^{2} \mathrm{D}_{\mathrm{M}, \mathrm{K}}^{\mathrm{J}}=\mathrm{J}(\mathrm{J}+1) \mathrm{D}_{\mathrm{M}, \mathrm{K}}^{\mathrm{J}}, \\
\mathrm{J}_{3} \mathrm{D}_{\mathrm{M}, \mathrm{K}}^{\mathrm{J}}=\mathrm{K} \mathrm{D}_{\mathrm{M}, \mathrm{K}}^{\mathrm{J}}, \\
\mathrm{J}_{\mathbf{2}} \mathrm{D}_{\mathrm{M}, \mathrm{K}}^{\mathrm{J}}=\mathrm{MD}_{\mathrm{M}, \mathrm{K}}^{\mathrm{J}},
\end{gathered}
$$

If $I_{1}=I_{2}$ then the collective wave function can be written

$$
\Psi=f_{J, r, \nu}(\beta)_{K, J, r} \mid \mathrm{MIK}>
$$

For ${ }^{20} \mathrm{Ne} \mathrm{K}$ is a good quantum number. The band structure of ${ }^{20} \mathrm{Ne}$ is shown in Figure [-1.

For a stiff nucleus with an axis of symmetry, so $I_{1}=I_{2}=I$, the rotational energy is given by

$$
\left.<\mathrm{JMK}\left|\sum_{\mathrm{k}} \frac{\mathrm{L}_{\mathrm{k}}^{2}}{2 \mathrm{I}_{\mathrm{x}}}\right| \mathrm{JMK}\right\rangle=\mathrm{H}^{2}\left[\frac{\mathrm{J}(\mathrm{J}+1)-\mathrm{K}^{2}}{2 \mathrm{I}}+\frac{\mathrm{K}^{2}}{2 \mathrm{I}_{3}}\right]
$$

ignoring the rotation-vibration coupling. For states in the same band the other contributions to the energy should be the same so 


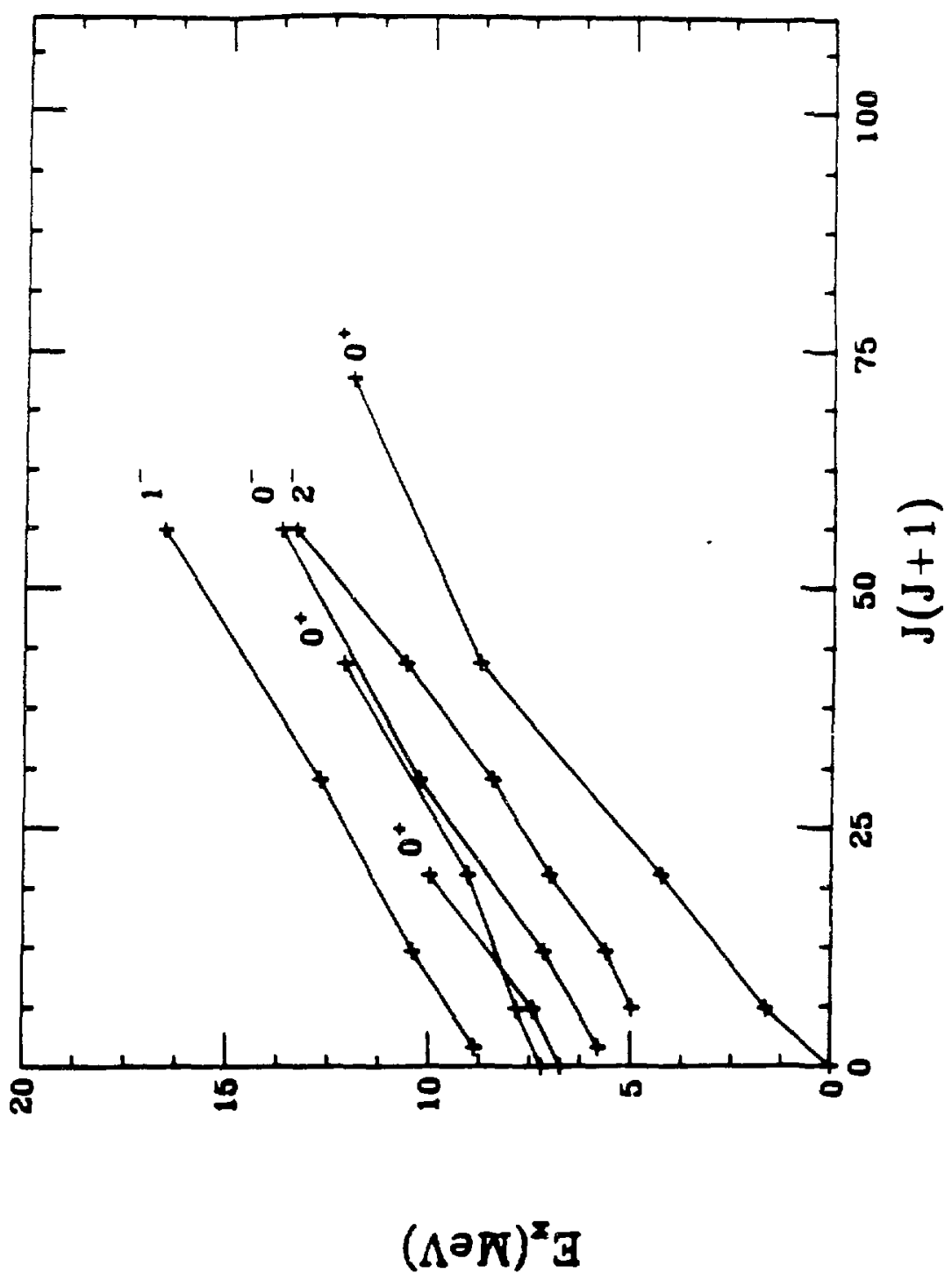

Figure III-1.

Excitation enerzies of the states in ${ }^{20}$ Ne plotted versus $J(J+1)$. States in the same band are connected with a line. 


$$
E_{J}=E_{0}+\frac{h^{2}}{2 I} J(J+1)
$$

Figure III-1 shows the excitation energies of the states of ${ }^{20} \mathrm{Ne}$ plotted versus $J(J+1)$. In general the contributions of $U_{1}$ and $U_{2}$ depend on $J^{2}$ and contribute to the energy in second order pertubation theory, so a term $-\mathrm{BJ}^{2}(\mathrm{~J}+1)^{2}$ is introduced giving

$$
E_{J}=E_{0}+\frac{n^{2}}{2 I} J(J+1)-B J^{2}(J+1)^{2}
$$

The results of fitting the bands of ${ }^{20} \mathrm{Ne}$ with these formulae are given in Table III1. The parameter $B$ is generally small and the values of $E_{0}$ and $A\left(A=H^{2} / 2 I\right)$ do not change much when the last term is added, demenstrating that the correction is small.

This model also makes some predictions about the relative strengths of the decays of states in the nucleus. In general electric quadrupole, E2, decays are the dominant decay mode so the quantity of interest is

$$
B\left(E 2, J_{1} K_{i}-J_{f} K_{f}\right)=\left(2 J_{1}+1\right)^{-1} \sum_{M_{i}, M_{f}}\left|<J_{f} M_{f} K_{l}\right| Q_{2 \mu}\left|J_{i} M_{i} K_{i}>\right|^{2}
$$

where in the space-fixed coordinates

$$
Q_{2 \mu}=e \sum_{k=1}^{Z} r_{k}^{2} Y_{2 \mu}\left(\theta_{k}\right)
$$

The intrinsic part of the wave function, $\chi$, has been ignored so far, but it will be necessary to include it in the discussion of the decays of excited states. For $K=0$ bands the wave function, including the intrinsic part, is

$$
\mid \mathrm{M} O>=\left(\frac{2 J+1}{8 \pi^{2}}\right)^{*} \mathrm{D}_{\mathrm{MO}}^{\mathrm{J}}\left(\theta_{1}\right) \times \mathrm{O}_{0}
$$

After some work, it can be shown (PR-75) that 
TABLE III-1.

Energy dependence of states in the rotational bands in ${ }^{20} \mathrm{Ne}^{1)}$.

$\begin{array}{llll}\mathrm{K}^{\pi} & \mathrm{E}_{0} & \mathrm{~A}^{2)} & \mathrm{B} \\ 0_{1}^{+} & 2.6 \times 10^{-4} & 0.272 & - \\ 0_{1}^{+} & 4.0 \times 10^{-5} & 0.288 & 2.61 \times 10^{-3} \\ 0_{2}^{+} & 6.54 & 0.147 & - \\ 0_{2}^{+} & 6.73 & 0.096 & -3.36 \times 10^{-3} \\ 0_{3}^{+} & 7.15 & 0.116 & - \\ 0_{3}^{+} & 7.20 & 0.100 & -4.066 \times 10^{-4} \\ 2^{-} & 4.12 & 0.141 & - \\ 2^{-} & 4.36 & 0.0935 & -1.24 \times 10^{-3} \\ 0^{-} & 5.43 & 0.144 & - \\ 0^{-} & 5.51 & 0.130 & -6.10 \times 10^{-4} \\ 1^{-} & 8.65 & 0.137 & - \\ 1^{-} & 8.59 & 0.149 & 3.55 \times 10^{-4}\end{array}$

1) Data from $A J-87$.

2) $\mathrm{A}=\frac{\mathrm{t}^{2}}{2 \mathrm{I}}$.

TABLE III-2.

$\left|\left\langle\chi_{K} \mid Q_{20}{ }^{\prime} \chi_{K}\right\rangle\right|$ for the ground state band in ${ }^{20} \mathrm{Ne}^{1}$.

Experimental Quantity $\left.\left.\quad\left|<\chi_{K}\right| Q_{20}{ }^{\prime}\right|_{\chi_{K}}\right\rangle \mid$

$\mathrm{B}(\mathrm{E} 2 \cdot 1.63-0.00)=67 \pm 4 \mathrm{e}^{2} \mathrm{fm}^{4} \quad 18.3 \pm 2.2$

$\mathrm{B}(\mathrm{E} 2+4.25-1.63)=72 \pm 7 \mathrm{e}^{2} \mathrm{fm}^{4} \quad 15.9 \pm 2.5$

$\mathrm{B}(\mathrm{E} 2+8.78-4.25)=65 \pm 10 \mathrm{e}^{2} \mathrm{fm}^{4} \quad 14.4 \pm 2.8$

$\mathrm{B}(\mathrm{E} 2+11.95-8.78)=30 \pm 4 \mathrm{e}^{2} \mathrm{fm}^{4} \quad 9.5 \pm 1.7$

$Q(1.63)=-27 \pm 3 \mathrm{efm}^{2} \quad 30 \pm 3$

1) Data from AJ-87 


$$
\mathrm{B}\left(\mathrm{E} 2, \mathrm{~J}_{1} \mathrm{O}-\mathrm{J} \mathrm{J}\right)=\left(\mathrm{J}_{1} 200 \mid \mathrm{J}_{\mathrm{O}} \mathrm{O}\right)^{2}\left|\left\langle\mathrm{x}_{0}\left|\mathrm{Q}^{\prime}{ }_{20}\right| \mathrm{x}_{0}\right\rangle\right|^{2} \text {, }
$$

where $Q^{\prime}{ }_{20}$ is $Q_{20}$ in the intrinsic, body-fixed frame. The quadrupole moment, $Q_{K}$, of a band in the space-fixed coordinates is

$$
Q_{K}=Q^{\prime}{ }_{K} \frac{3 K^{2}-J(J+1)}{(J+1)(2 J+3)},
$$

where $Q^{\prime}{ }_{K}$ is the intrinsic quadrupole moment in the body-fixed coordinates for a band $K$ and is defined by

$$
Q_{K}^{\prime}=\left(\frac{16 \pi}{5}\right)^{1 / 2}<\chi_{K}\left|Q_{20}^{\prime}\right|_{\chi_{K}}>
$$

Therefore, all the decay strengths within a band, $K$, and the intrinsic quadrupole moment of the band are simply related to one quantity, $\left\langle\chi_{K}\left|Q^{\prime}{ }_{20}\right| \chi_{K}\right\rangle$. The experimental results for the ground state $K^{\pi}=0^{+}$band in ${ }^{20} \mathrm{Ne}$ are compared with this result in Table III-2. The value obtained from the quadrupole moment is about twice the value from the E2 transitions, but in general the agreement is very good.

Within a band the ratios of the transition strengths are equal to the ratios of Clebsch-Gordans. The transition strengths for transitions within the band for the ground state band in ${ }^{20} \mathrm{Ne}$ were given in Table III-2 and for other bands are given in Table III-3. If the intrinsic structure of different bands is different then transitions between bands should be much weaker. The known strengths for some cross-band transitions are given in Table III-4. There are some very large crossband transitions, implying either a large amount of band mixing or the structures of some bands are similar. The transitions between states of two given bands are of approximately the same magnitude implying that the transitions are still proportional to a matrix element between the intrinsic states, and that the structure 
TABLE III-3.

In-band transition strengths in ${ }^{20} \mathrm{Ne}^{1,2)}$.

$\begin{array}{llllll}\mathrm{K}^{\pi} & \mathrm{J}_{\mathrm{i}}{ }^{\pi} & \mathrm{J}_{\mathrm{f}}{ }^{\pi} & \lambda & \mathrm{B}(\mathrm{E \lambda \lrcorner})\left(\mathrm{e}^{\wedge} \mathrm{fm}^{2 \lambda}\right) & \mathrm{B}(\mathrm{E} \lambda\lrcorner)(\mathrm{W} . \mathrm{U} .) \\ 2^{-} & 3^{-} & 2^{-} & 2 & 123 \pm 53 & 7.6 \pm 3.3 \\ & 4^{-} & 2^{-} & 2 & 5.64 & 0.35 \\ & 4^{-} & 3^{-} & 2 & 91 \pm 11 & 5.7 \pm 0.7 \\ & 5^{-} & 3^{-} & 2 & 88 \pm 21 & 5.5 \pm 1.3 \\ 0^{-} & 3^{-} & 1^{-} & 2 & 165 \pm 28 & 10.2 \pm 1.7\end{array}$

1) Transition strengths for the ground state band are in Table III-2.

${ }^{2)}$ Data from AJ-87.

TABLE III-4.

Cross-band transition strengths in ${ }^{20} \mathrm{Ne}^{1)}$.

$\begin{array}{llllll}\mathrm{K}_{1}{ }^{\pi} & \mathrm{K}_{\mathrm{f}}{ }^{\pi} & \mathrm{J}_{1}{ }^{\pi} & \mathrm{J}_{\mathrm{f}}{ }^{\pi} & \lambda & \mathrm{B}(\mathrm{E \lambda} \downarrow)\left(\mathrm{e}^{\lambda} \mathrm{fm}^{2 \lambda}\right) \\ 2^{-} & 0_{1}{ }^{+} & 3^{-} & 0^{+} & 3 & 270 \pm 90 \\ & & 3^{-} & 2^{+} & 1 & (3.2 \pm 0.9) \times 10^{-6} \\ & & 4^{-} & 2^{+} & 3 & 144 \pm 67 \\ & & 4^{-} & 4^{+} & 1 & (4.33 \pm 0.15) \times 10^{-5} \\ 0^{-} & 0_{1}^{+} & 3^{-} & 4^{+} & 1 & (3.8 \pm 0.4) \times 10^{-5} \\ 0_{2}^{+} & 0_{1}^{+} & 2^{+} & 0^{+} & 2 & 0.16 \pm 0.03 \\ & & 2^{+} & 2^{+} & 2 & 5.5 \pm 0.8 \\ & & 4^{+} & 2^{+} & 2 & 27 \pm 12 \\ 0_{3}^{+} & 0_{1}^{+} & 2^{+} & 0^{+} & 2 & 2.4 \pm 0.3 \\ & & 2^{+} & 2^{+} & 2 & 1.6 \pm 0.2 \\ & & 2^{+} & 4^{+} & 2 & 4.2 \\ & & 4^{+} & 2^{+} & 2 & 19 \pm 2\end{array}$

1) Data from AJ-87. If no mixing ratio was given in the compilation, the lowest electric multipole was assumed. 
TABLE III-5.

\begin{tabular}{lllll}
\multicolumn{5}{c}{$\alpha$ decays of states in ${ }^{20} \mathrm{Ne}^{1)}$} \\
$\mathrm{K}^{\pi}$ & $J^{\pi}$ & $\operatorname{Ex}(\mathrm{MeV})$ & $\Gamma_{\alpha}$ & $\Gamma_{\alpha} / \Gamma_{\text {s.p. }}$ \\
$0^{+}$ & $6^{+}$ & 8.78 & $110 \pm 25 \mathrm{eV}$ & 0.21 \\
& $8^{+}$ & 11.99 & $35 \pm 10$ & 0.10 \\
$0_{2}^{+}$ & $0^{+}$ & 6.72 & $15 \pm 7 \mathrm{keV}$ & 0.33 \\
& $2^{+}$ & 7.42 & 8 & 0.10 \\
& $4^{+}$ & 9.99 & $150 \pm 50$ & 0.33 \\
$0_{3}^{+}$ & $0^{+}$ & 7.20 & $4 \mathrm{keV}$ & 0.017 \\
& $2^{+}$ & 7.83 & 2.4 & 0.008 \\
& $4^{+}$ & 9.04 & 3.2 & 0.030 \\
& $6^{+}$ & 12.14 & 0.13 & 0.001 \\
$0_{4}^{+}$ & $0^{+}$ & 8.4 & $\approx 800 \mathrm{keV}$ & $\approx 0.43$ \\
& $2^{+}$ & 8.8 & $\approx 800$ & $\approx 0.64$ \\
& $4^{+}$ & 10.8 & 350 & 0.50 \\
$6^{+}$ & 12.59 & 150 & 0.56
\end{tabular}

1) Data from PI-78. 
of the intrinsic state is relatively constant throughout the band. Also, if the intrinsic structure of the states within a band is the same, the states should have similar $\alpha$-decay widths to the same final state after all kinematic factors and penetrabilities have been removed. These are given in Table III-5 for the $\mathrm{K}^{\pi}=0^{+}$bands in ${ }^{20} \mathrm{Ne}$, states below $4.73 \mathrm{MeV}$ excitation cannot $\alpha$ decay.

The rotational model describes ${ }^{20} \mathrm{Ne}$ very well. It has a well-developed band structure; its first 14 states, up to $8.5 \mathrm{MeV}$ in excitation, can all be reasonably placed in rotational bands. The energies of its excited states roughly follow the expected systematics, and its excited states have significantly stronger in-band decays than cross-band decays. Of course this band structure is not perfect; there is evidence of mixing between bands (FO-72,FO-76), which will be discussed, as needed, in later chapters.

\section{III-2. Shell Mode!}

Another basic model of the nucleus is the shell model (PR-75 gives a good introduction to the shell model, LA- 80 gives a more complete treatment of the shell model, BE-72,BR-77). Unlike collective models such as the rotational model just described the shell model deals explicitly with nucleons, with the intrinsic structure of the nucleus in a manner similar to the Bohr model of the atom. The simplest form of the shell model starts with a Hamiltonian:

$$
\mathrm{H}=\mathrm{T}+\mathrm{V} \text {, }
$$

where $\mathrm{T}$ is the kinetic energy given by

$$
\mathrm{T}=\sum_{\mathrm{i}=1}^{\mathrm{A}} \frac{\mathrm{p}_{\mathrm{i}}{ }^{2}}{2 \mathrm{~m}_{\mathrm{i}}}
$$




$$
\mathrm{V}=\sum_{\mathrm{i}=1}^{\mathrm{A}} \mathrm{V}_{\mathrm{l}}
$$

where $V_{1}$ is a central potential felt by all the nucleons. A spin-orbit potential is normally included. Usually $V_{1}$ is taken to be either a harmonic oscillator or a Woods-Saxon potential. Figure III-2 shows the energy levels found using reasonable strengths for the potentials. In this simple model, nuclear structure is simply a matter of filling shells from the bottom up. However, without interactions between particles there is a large degeneracy between states, which is not observed experimentally. For example, ${ }^{20} \mathrm{Ne}$ is $(0 \mathrm{~s} 1 / 2)^{4}(0 \mathrm{p} 3 / 2)^{8}(0 \mathrm{pl} / 2)^{4}(0 \mathrm{~d} 5 / 2)^{4}$ in its ground state. This configuration can have $J=0$ to 8 , and without some interaction between particles all these states are degenerate. If the choice of the central potential is good enough then the interaction between the particles can be treated as a perturbation. With these interactions the Hamiltonian becomes

$$
\mathrm{H}=\mathrm{H}_{0}+\sum_{\mathrm{i}=1 \mathrm{j}=1}^{A} \sum_{\mathrm{i}, \mathrm{j}}^{\mathrm{A}} \mathrm{v}_{\mathrm{j}}
$$

where $\mathrm{H}_{0}$ is the original Hamiltonian and $v_{i, j}$ is the interaction between particles. It is also possible to include 3-body and higher-order interactions, but they are not needed. One method of determining the $v_{i, J}$ is to calculate them from the fundamental nucleon-nucleon interaction. Another method is to vary them to fit known states. All of the calculations presented here will use interactions derived by fitting known states.

Two shell-model calculations of the structure of ${ }^{20} \mathrm{Ne}$ were done. Both calculations were done with the computer code OXBASH (OX-85). The first calculation, (sd) ${ }^{4}$, assumed all levels up to $0 \mathrm{p} 1 / 2$ were filled, i.e. an ${ }^{10} \mathrm{O}$ closed core and 4 particles anywhere in the sd-shell, with B. H. Wildenthal's usd interaction (WI-84). The second calculation, ZBM, assumed all levels up to $0 \mathrm{p} 3 / 2$ were filled, i.e. a ${ }^{12} \mathrm{C}$ 


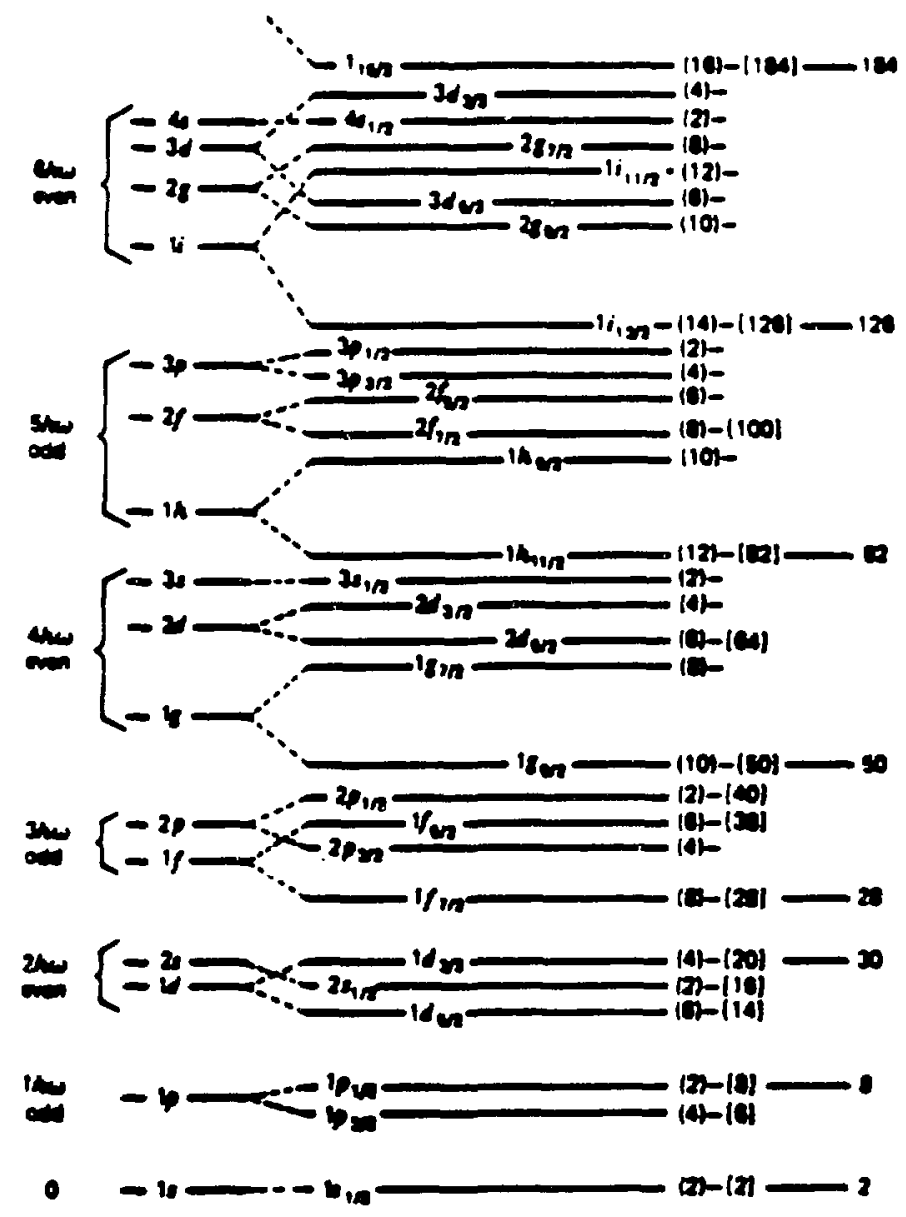

Figure III-2.

Approximate level pattern from the shell model (SH-74). 
closed core and 8 particles anywhere in the $0 \mathrm{p} 1 / 2,0 \mathrm{~d} 5 / 2,1 \mathrm{~s} 1 / 2$ sub-shells. Zuker, Buck, and McGrory, first used this model space to explain the structure of ${ }^{10} \mathrm{O}$ (ZL-68). It used the F interaction from MicGrory and Wildenthal (Mc-73). In the rest of this section the results of these calculations will be compared with experimental results to determine which states in ${ }^{20} \mathrm{Ne}$ they describe. Also, since ${ }^{20} \mathrm{Ve}$ has been shown to be a good rotational nucleus, the rotational model will also be applied to the shell-model states.

The (sd) calculation is by far the simpler of the two. The states found in this model are shown with the known positive-parity states of ${ }^{20} \mathrm{Ne}$ in Figure III-3. Because this model space can create only positive-parity states, the negative-parity states were not included. Calculated transition rates are compared with known transition rates in Table III-6. There are no unnatural-parity states below $10 \mathrm{MeV}$ in the calculation, which agrees with what is known experimentally. However, the calculation predicts only two $0^{+}, 2^{+}$, and $4^{+}$states below $10 \mathrm{MeV}$, while there are four $0^{+}$and $2^{+}$states and three $4^{+}$states known. The additional states and the known negative-parity states result either from core excitation, i.e. particles excited out of the ${ }^{10} \mathrm{O}$ core, or the excitation of particles out of the sd shell into the fp shell. The calculation agrees reasonably well for the ground-state band, but not as well for the first excited band. This may be due to mixing between the $\mathrm{O}_{2}^{+}$band and the $\mathrm{O}_{3}^{+}$or $\mathrm{O}_{4}^{+}$band. The low-lying states in this calculation can easily be grouped into two bands. Figure III-4 shows their calculated energies plotted versus $j(J+1)$, Table III-7 gives the results of a fit to the energy formula found for rotational bands, and Table III-8 gives the calculated value of $\left\langle x_{K}\left|Q^{\prime}{ }_{20}\right| x_{K}\right\rangle$ for both bands. The ground-state band in the calculation follows the rotational-model systematics very well, possibly better than the band really does. On the other hand the excited band does not follow them very well. 
$.51-$

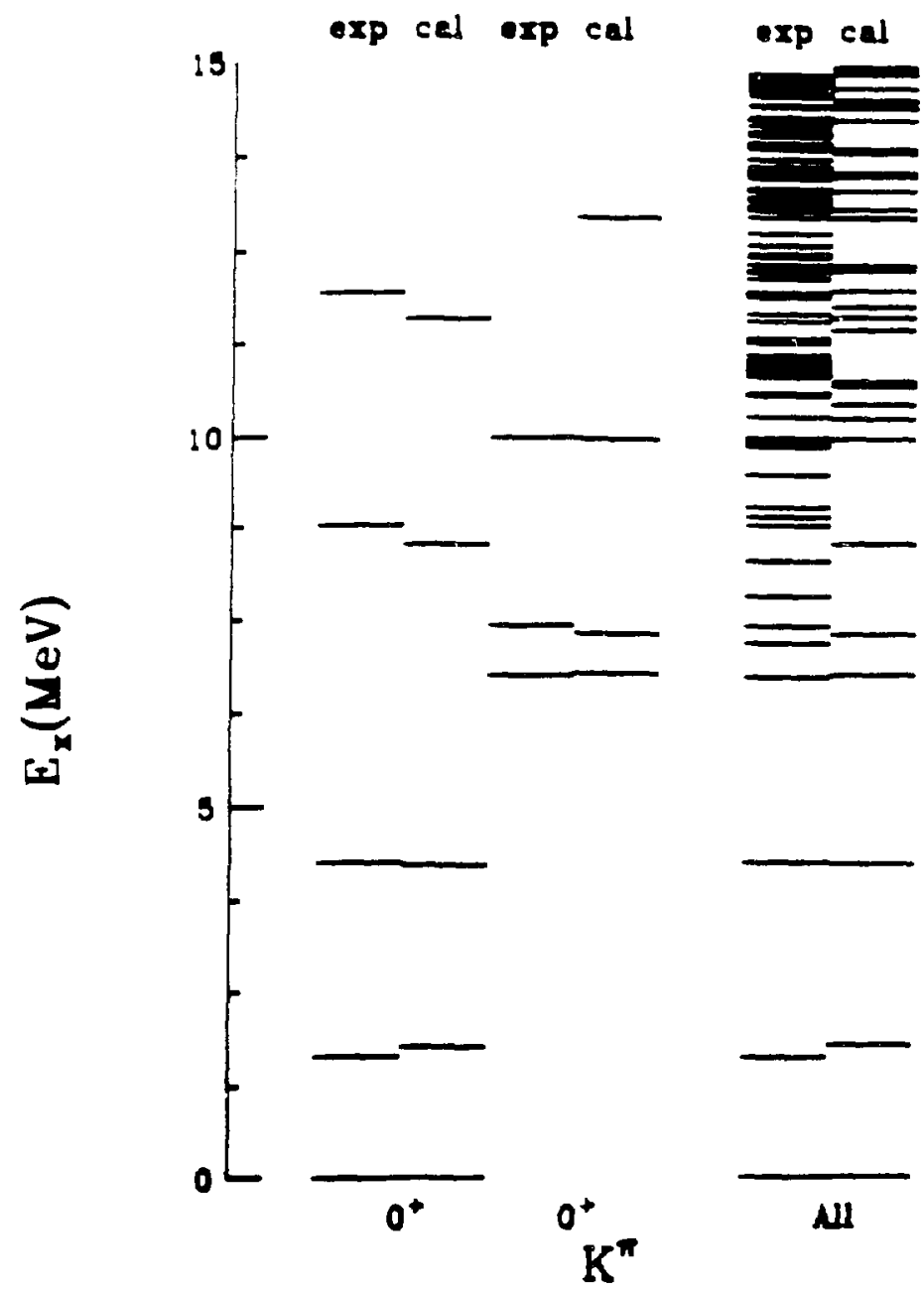

Figure III-3.

Energies calculated in the (sd) model space and the observed energies of positive-parity states in ${ }^{20} \mathrm{Ne}$. 
TABLE III-6.

Comparison of (sd) calculated $B(E 2)$ 's with experimental $B(E 2)$ 's.

$\begin{array}{llll}\mathrm{J}_{1}{ }^{\pi} & \mathrm{J}_{\mathrm{f}}{ }^{\pi} & \mathrm{B}(\mathrm{E} 2)_{\text {theo }}\left(\mathrm{e}^{2} \mathrm{fm}^{4}\right) & \mathrm{B}(\mathrm{E} 2)_{\text {exp }}\left(\mathrm{e}^{2} \mathrm{fm}\right. \\ 2_{1}{ }^{+} & 0_{1}{ }^{+} & 60.6 & 67 \pm 4 \\ 4_{1}{ }^{+} & 2_{1}{ }^{+} & 72.2 & 72 \pm 7 \\ 6_{1}{ }^{+} & {4_{1}}^{+} & 55.9 & 65 \pm 10 \\ 8_{1}{ }^{+} & 6_{1}{ }^{+} & 36.4 & 30 \pm 4 \\ 0_{2}{ }^{+} & 2_{1}{ }^{+} & 9.74 & i 2 \\ 2_{2}{ }^{+} & 0_{1}{ }^{+} & 0.033 & \leq 0.16 \pm 0.03 \\ 2_{2}{ }^{+} & 2_{1}{ }^{+} & 4.42 & 5.5 \pm 0.8 \\ 4_{3}{ }^{+} & 2_{1}{ }^{+} & 2.73 & 27 \pm 12\end{array}$

1) Data from AJ-87 


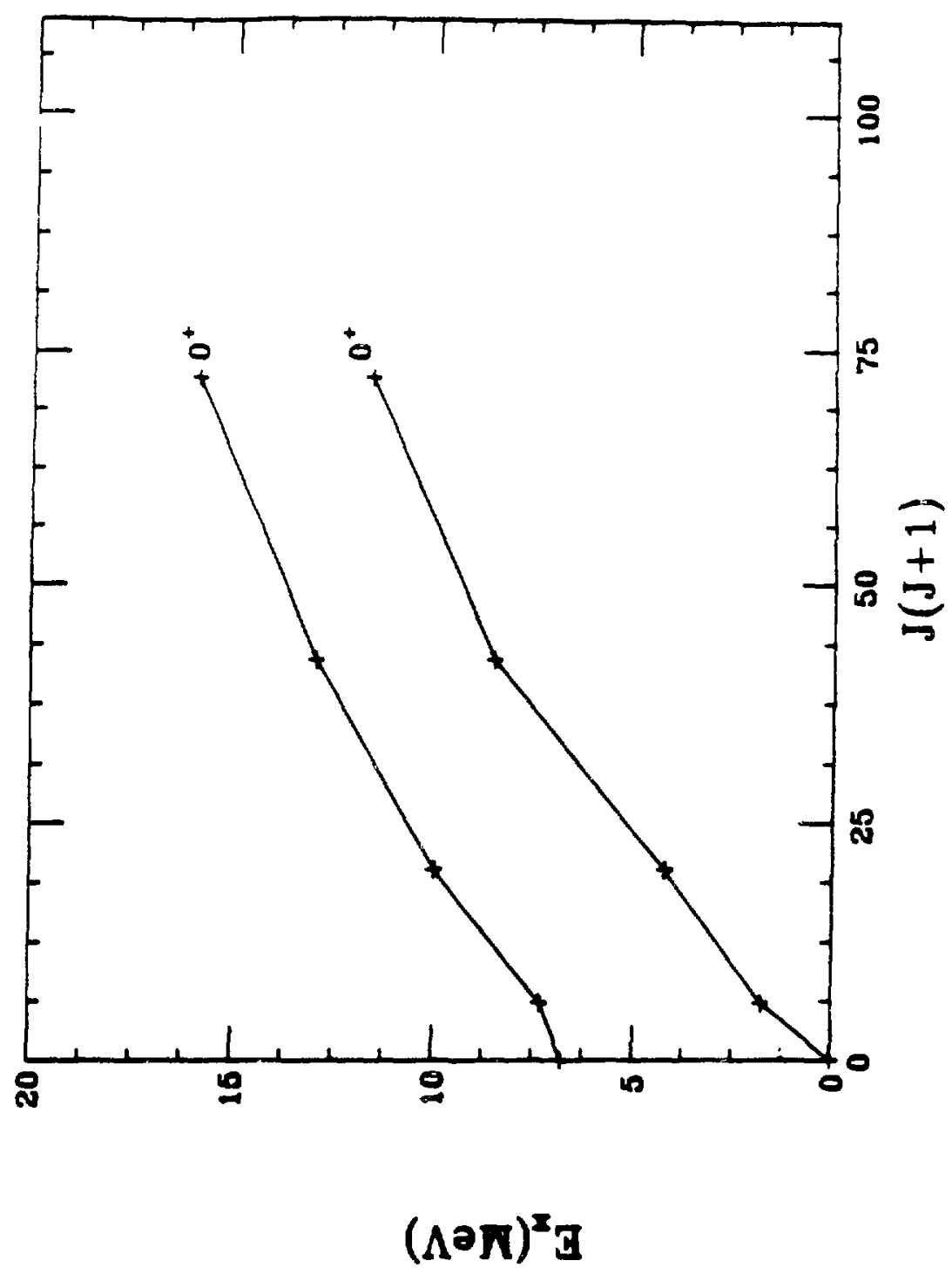

Figure III-4.

Energies calculated in the (sd) model space plotted versus $\mathrm{J}(\mathrm{J}+1)$. States in the same band are connected with a solid line. 
T ABLE III-7.

Energy dependence of states in the rotational bands from the (sd) calculation.

$K^{\pi} \quad E_{0}$

$A^{1)}$

$\mathrm{B}$

$0_{1}^{+} 7.22 \times 10^{-1}$

0.161

$0_{1}^{+} 8.11 \times 10^{-2}$

0.247

$1.20 \times 10^{-3}$

$\mathrm{O}_{2}{ }^{+} 6.93$

0.130

$\mathrm{O}_{2}^{+} \quad 6.55$

0.182

$7.18 \times 10^{-4}$

1) $A=\frac{H^{2}}{2 I}$.

T ABLE III-8.

$\left|<\chi_{K}\right| Q_{20}{ }^{\prime} \chi_{K}>\mid$ for the $K^{\pi}=0^{+}$bands in ${ }^{20} \mathrm{Ne}$ from the (sd) calculation. Experimental Quantity $\left|<x_{K}\right| Q_{20}{ }^{\prime}\left|x_{K}>\right|$

$\mathrm{K}^{\pi}=0_{1}^{+}$

$\mathrm{B}\left(\mathrm{E} 2 \downarrow 2^{+}-0^{+}\right)=60.6 \mathrm{e}^{2} \mathrm{fm}^{4}$

17.4

$\mathrm{B}\left(\mathrm{E} 2+4^{+}-2^{+}\right)=72.2 \mathrm{e}^{2} \mathrm{fm}^{4}$

15.9

$\mathrm{B}\left(\mathrm{E} 2+6^{+}-4^{+}\right)=55.9 \mathrm{e}^{2} \mathrm{fm}^{4}$

13.3

$\mathrm{B}\left(\mathrm{E} 2 b^{+} \rightarrow 6^{+}\right)=36.4 \mathrm{e}^{2} \mathrm{fm}^{4}$

10.5

$\mathrm{Q}\left(2^{+}\right)=-15.8 \mathrm{efm} \mathrm{m}^{2}$

17.4

$\mathrm{K}^{\pi}=\mathrm{O}_{2}^{+}$

$\mathrm{B}\left(\mathrm{E} 2 \cdot 2^{+}-0^{+}\right)=17.0 \mathrm{e}^{2} \mathrm{fm}^{4}$

9.22

$\mathrm{B}\left(\mathrm{E} 2 \downarrow 4^{+}-2^{+}\right)=7.21 \mathrm{e}^{2} \mathrm{fm}^{4}$

5.02

$\mathrm{B}\left(\mathrm{E} 2 \cdot 6^{+}-4^{+}\right)=54.39 \mathrm{e}^{2} \mathrm{fm}^{4}$

13.1

$\mathrm{B}\left(\mathrm{E} 2 \downarrow 8^{+} \rightarrow 6^{+}\right)=20.3 \mathrm{e}^{2} \mathrm{fm}^{4}$

7.85

$\mathrm{Q}\left(2^{+}\right)=10.09 \mathrm{efm}^{2}$

11.1 
The second calculation includes the $\mathrm{p} 1 / 2$ orbit, but excludes the $d 3 / 2$ orbit. It is a more complicated calculation because there are now 8 active particles instead of 4 . Including the p1/2 orbit allows negative-parity states. States found in this model are compared with the known states in Figures III-5 and III-6. Calculated transition rates are compared with known transition rates in Table III-9. In this calculation we have another $0^{+}, 2^{+}$, and $4^{+}$state below $10 \mathrm{MeV}$. This extra set of states looks like a $6 \mathrm{p}-2 \mathrm{~h}$ band, and appears to correspond to the $\mathrm{K}^{\pi}=\mathrm{O}_{3}^{*}$ band. This band is believed to be an $8 \mathrm{p}-4 \mathrm{~h}$ band. Unfortunately the identification of this band in the calculation is not as easy as in the experimental data. The second and third $4^{+}, 6^{+}$, and $8^{+}$states in the calculation are very similar. Experimentally the bands cross between their $2^{+}$and $4^{+}$members. The calculation is not very consistent with rotational band structure for these two bands. The states have been analyzed both ways, assuming they do cross as is the case experimentally, and assuming they do not cross as was assumed by McGrory and Wildenthal (Mc-73). The ground-state band in the calculation keeps its rotational qualities. The excitation energieg of these bands are plotted versus $J(J+1)$ in Figure III-7, the coefficients of a fit with the rotational energy formula are given in Table III-10, and a comparison of $\left\langle\chi_{K}\left|Q^{\prime}{ }_{20}\right| \chi_{K}\right\rangle$ is given in Table III-11.

The ZBM calculation also gives a large number of negative-parity states, although the correspondence with the experimental spectrum is not as good as with the positive-parity states. The experimental $2^{-}$band is obviousiy in the calculation and it is possible to pick out a $0^{-}$band. However, the $0^{-}$band in ${ }^{20} \mathrm{Ne}$ is believed to be an $(s d)^{3}(f p)$ band, not a $p^{-1}(s d)^{6}$ band. There may also be a $1^{-}$ band in ${ }^{20} \mathrm{Ne}$ starting at $8.854 \mathrm{MeV}$, of similar structure to the $2^{-}$band. This seems to explain the calculated states better, although the energies disagree by approximately $2.5 \mathrm{MeV}$ with experiment. The negative-parity bands have been 


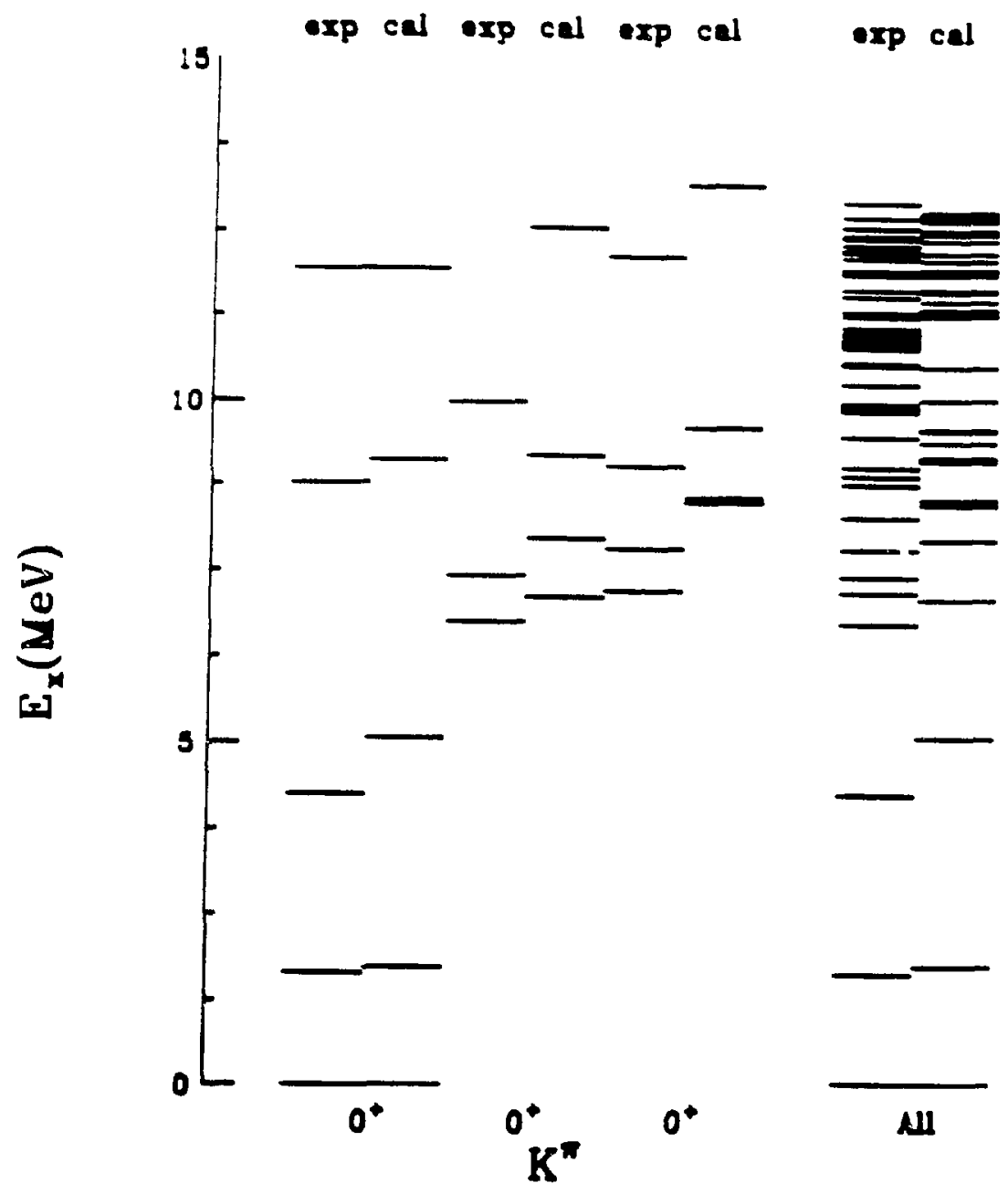

Figure III-5.

Energies calculated in the ZBM model space and the observed energies of positive-parity states in ${ }^{20} \mathrm{Ne}$. 


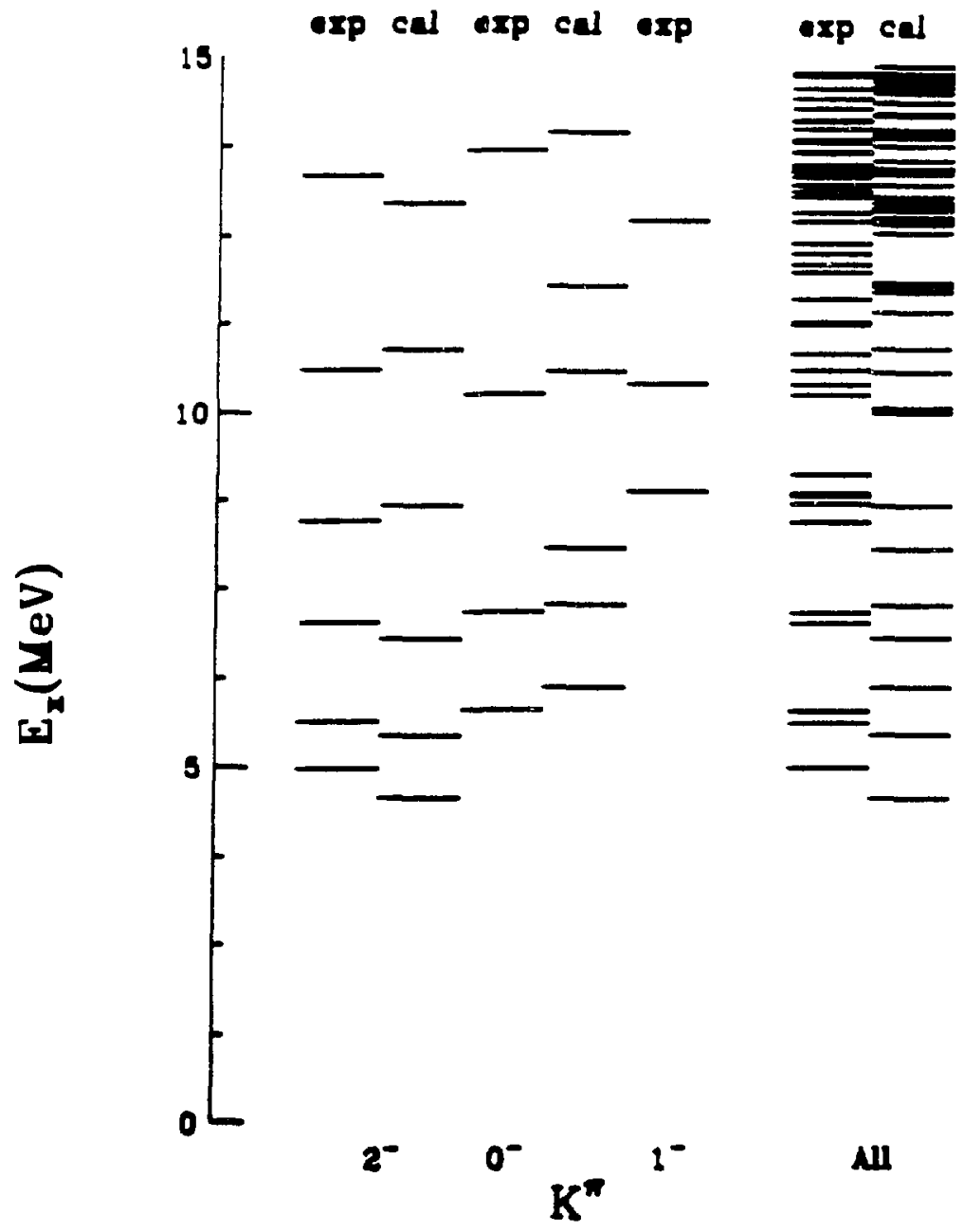

Figure III-6.

Energies calculated in the ZBM model space and the observed energies of negative-parity states in ${ }^{20} \mathrm{Ne}$. 
TABLE III-9.

Comparison of ZBM calculated $B(E \lambda)$ 's with experimental $B(E \lambda)$ 's.

\begin{tabular}{|c|c|c|c|}
\hline $\mathrm{J}_{\mathrm{f}}{ }^{\pi}$ & $B(E \lambda !)\left(e^{\lambda} f m^{2 \lambda}\right)_{\text {theo }}$ & $B(E \lambda !)\left(e^{\lambda} f m^{2 \lambda}\right)_{\exp }^{1)}$ & $\lambda$ \\
\hline $2_{1}^{+} \quad 0_{1}^{+}$ & 46.7 & $67 \pm 4$ & 2 \\
\hline $2_{1}^{+}$ & 53.3 & $72 \pm 7$ & 2 \\
\hline $4_{1}^{+}$ & 33.0 & $65 \pm 10$ & 2 \\
\hline $6_{1}^{+}$ & 18.3 & $30 \pm 4$ & 2 \\
\hline $2_{1}^{+}$ & 0.143 & 12 & 2 \\
\hline $0_{1}^{+}$ & 0.051 & $\leq 0.16 \pm 0.03$ & 2 \\
\hline $2_{1}^{+}$ & 0.144 & $5.5 \pm 0.8$ & 2 \\
\hline $2_{1}^{+}$ & 0.095 & $27 \pm 12$ & 2 \\
\hline $2_{1}^{+}$ & 0.374 & $1.01 \pm 0.18$ & 2 \\
\hline $0_{1}^{+}$ & 0.905 & $2.4 \pm 0.3$ & 2 \\
\hline $2_{1}^{+}$ & 0.174 & $1.6 \pm 0.2$ & 2 \\
\hline $4_{1}^{+}$ & 1.24 & $<4.2$ & 2 \\
\hline $2_{1}^{+}$ & 2.98 & $19 \pm 2$ & 2 \\
\hline $4_{1}^{+}$ & 1.42 & $<3.4$ & 2 \\
\hline $2_{1}^{-}$ & 72.9 & $12 \pm 5$ & 2 \\
\hline $2_{1}^{-}$ & 25.5 & $5.6 \pm 0.4$ & 2 \\
\hline $3_{1}^{-}$ & 57.0 & $91 \pm 11$ & 2 \\
\hline $1_{1}^{-}$ & 4.25 & $165 \pm 28$ & 2 \\
\hline $3_{1}^{-}$ & 32.6 & $88 \pm 21$ & 2 \\
\hline $2_{1}^{+}$ & $8.56 \times 10^{-3}$ & $(3.6 \pm 0.7) \times 10^{-0}$ & 1 \\
\hline $2_{1}^{+}$ & $6.05 \times 10^{-2}$ & $15 \pm 3$ & 3 \\
\hline $0_{1}^{+}$ & 164 & $270 \pm 90$ & 3 \\
\hline $2_{1}^{+}$ & $4.94 \times 10^{-2}$ & $3.2 \pm 0.9 \times 10^{-0}$ & $1^{2)}$ \\
\hline $2_{1}^{+}$ & 1.14 & $3.4 \pm 1.0 \times 10^{4}$ & $3^{3)}$ \\
\hline $0_{1}^{+}$ & $1.04 \times 10^{-3}$ & $3.9 \pm 1.5 \times 10^{-6}$ & $1^{2)}$ \\
\hline $2_{1}^{+}$ & $1.02 \times 10^{-2}$ & $5.5 \pm 1.1 \times 10^{-5}$ & $1^{2)}$ \\
\hline $2_{1}^{+}$ & 61.4 & $4.7 \pm 1.0 \times 10^{5}$ & $3^{2)}$ \\
\hline $21^{+}$ & 206 & $144 \pm 67$ & 3 \\
\hline $4_{1}^{+}$ & $3.41 \times 10^{-2}$ & $4.33 \pm 0.15 \times 10^{-5}$ & $1^{2)}$ \\
\hline $4_{1}^{+}$ & 30.7 & $2.08 \pm 0.17 \times 10^{\circ}$ & $3^{3)}$ \\
\hline $4_{1}^{+}$ & $4.75 \times 10^{-2}$ & $3.7 \pm 0.4 \times 10^{-5}$ & $1^{2)}$ \\
\hline $4_{1}^{+}$ & -12.7 & $1.46 \pm 0.17 \times 10^{6}$ & $3^{3)}$ \\
\hline
\end{tabular}


TABLE III-9.

Comparison of $2 B M$ calculated $B(E \lambda)$ 's with experimental $B(E \lambda)$ 's.

$\begin{array}{lllll}\mathrm{J}_{1}{ }^{\pi} & \mathrm{J}_{\mathrm{f}}{ }^{\pi} & \mathrm{B}(\mathrm{E} \lambda \downarrow)\left(\mathrm{e}^{\lambda} \mathrm{fm}^{2 \lambda}\right)_{\text {theo }} & \mathrm{B}(\mathrm{E} \lambda \downarrow)\left(\mathrm{e}^{\lambda} \mathrm{fm}^{2 \lambda}\right)_{\exp }{ }^{1)} & \lambda \\ 1_{2}{ }^{-} & 0_{1}{ }^{+} & 0.249 & 9 \pm 2 \times 10^{-5} & 1^{2)} \\ 1_{2}^{-} & 2_{1}{ }^{+} & 1.34 \times 10^{-3} & 2.46 \times 10^{-5} & 1^{2)} \\ 1_{2}{ }^{-} & 2_{1}{ }^{+} & 7.20 & 2.7 \pm 1.8 \times 10^{4} & 3^{\text {j) }}\end{array}$

1) Data from AJ-87.

${ }^{2)}$ Assuming the transition is $100 \% \mathrm{E} 1$.

3) Assuming the transition is $100 \% \mathrm{E3}$. 


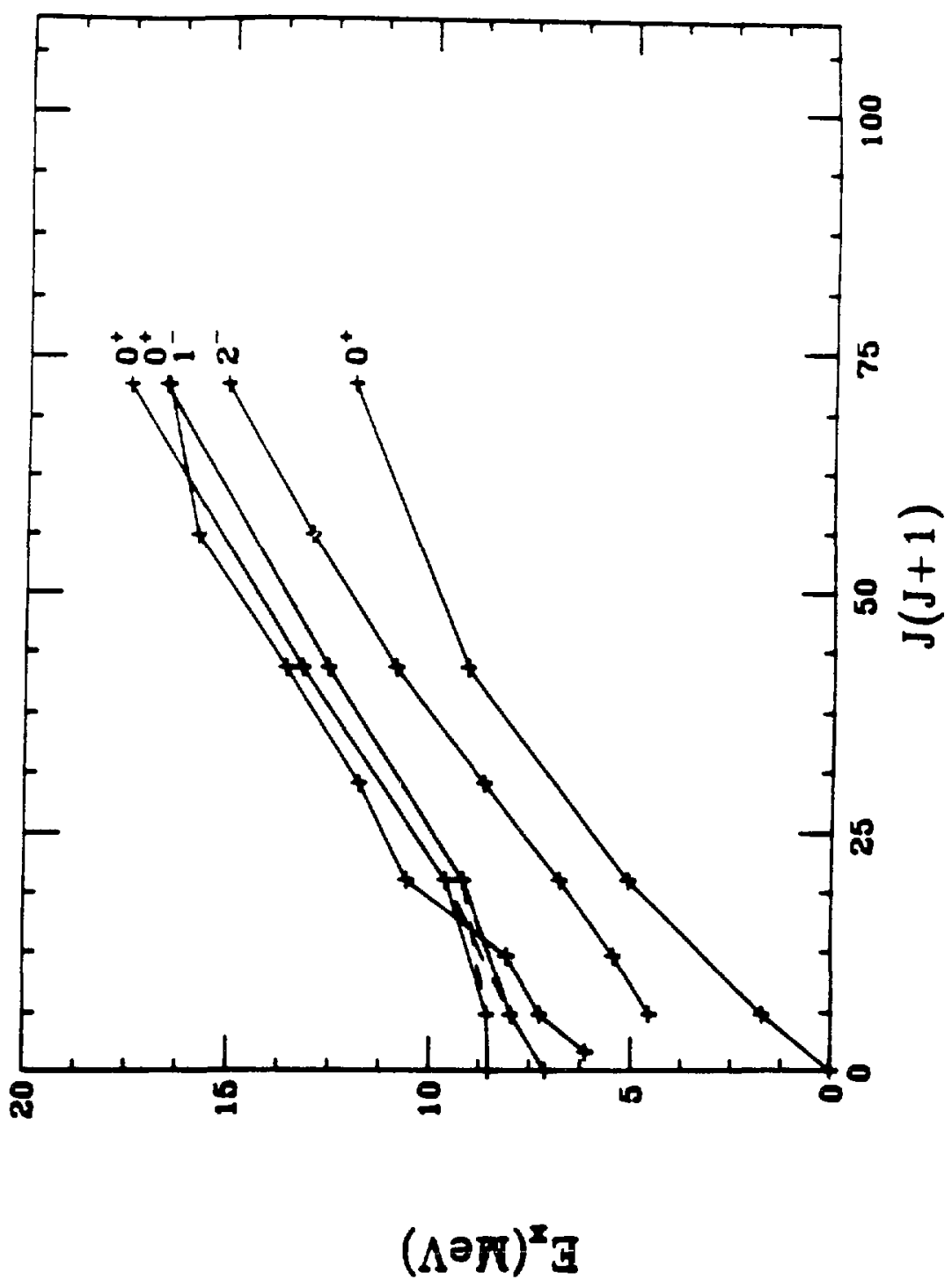

Figure III-7.

Energies calculated in the ZBM model space plotted versus $J(J+1)$. States in the same band are connected with a solid line. The solid line connecting the states in the $\mathrm{O}_{9}^{+}$and $\mathrm{O}_{3}^{+}$bands anumes they do not crom. The dashed line connecting the states in the $\mathrm{O}_{2}^{+}$and $\mathrm{O}_{3}^{+}$bands asoumes they do crom. 
TABLE III- 10.

Energy dependence of states in the rotational bands from the ZBM calculation.

$\mathrm{K}^{\pi} \quad E_{0}$

$0_{1}^{+} \quad 9.29 \times 10^{-1}$

$0_{1}^{+} \quad 2.54 \times 10^{-2}$

$\mathrm{O}_{2}{ }^{-2)} \quad 6.97$

$\mathrm{O}_{2}{ }^{+21} \quad 7.11$

$0_{3}^{+2)} \quad 7.82$

$0_{3}{ }^{+2)} \quad 8.22$

$\mathrm{O}_{2}{ }^{+3)} \quad 7.00$

$\mathrm{O}_{2}^{+31} \quad 7.07$

$\mathrm{O}_{3}{ }^{+3)} \quad 7.79$

$0_{3}{ }^{+3)} \quad 8.26$

$2_{1}^{-} \quad 3.61$

$2_{1}^{-} \quad 3.20$

$0_{1}^{-4)} \quad 6.02$

$0_{1}^{-4)} \quad 5.54$

$1_{1}^{-4)} 5.98$

$1_{1}^{-4)} \quad 5.63$

1) $A=\frac{t^{2}}{2 I}$.

2) Assuming the $\mathrm{O}_{2}^{+}$and $\mathrm{O}_{3}^{+}$bands do not cross.

3) Assuming the $\mathrm{O}_{2}^{+}$and $\mathrm{O}_{3}^{+}$bands do cross.

4) see text about $\mathrm{O}_{1}^{-}$and ${1_{1}^{-}}^{-}$bands in the $\mathrm{ZBM}$ calculation.

TABLE III-11.

$\left.\left.\left|<\chi_{K}\right| Q_{20}\right|_{\chi_{K}}\right\rangle \mid$ for the $K^{\pi}=0^{+}$bands in ${ }^{20} \mathrm{Ne}$ from the $2 \mathrm{BM}$ calculation.

Experimental Quantity

$$
\left|<\chi_{\mathbf{K}}\right| Q_{20}{ }^{\prime} \mid \chi_{K}>1
$$

$\mathrm{K}^{\pi}=\mathrm{O}_{3}^{+}$

$\mathrm{B}\left(\mathrm{E} 2 \cdot \mathrm{2}^{+} \rightarrow 0^{+}\right)=46.7 \mathrm{e}^{2} \mathrm{fm}^{4}$

15.3

$\mathrm{B}\left(\mathrm{E} 2 \cdot 4^{+}-2^{+}\right)=53.3 \mathrm{e}^{2} \mathrm{fm}^{4}$

13.7

$\mathrm{B}\left(\mathrm{E} 2 \mathrm{i}^{+} \mathrm{6}^{+} 4^{+}\right)=33.0 \mathrm{e}^{2} \mathrm{fm}^{4}$

10.2

$\mathrm{B}\left(\mathrm{E} 2 ! 8^{+} \rightarrow 6^{+}\right)=18.3 \mathrm{e}^{2} \mathrm{fm}^{4}$

7.45

$\mathrm{Q}\left(2^{+}\right)=-13.21 \mathrm{efm}^{2}$
$B$

$1.69 \times 10^{-3}$

$-2.66 \times 10^{-4}$

$-7.38 \times 10^{-4}$

$-1.31 \times 10^{-4}$

$-8.73 \times 10^{-4}$

$4.28 \times 10^{-4}$

$1.11 \times 10^{-3}$

$1.31 \times 10^{-3}$ 
TABLE III-11.

$\left|<\chi_{K}\right| Q_{20}{ }^{\prime} \chi_{K}>\mid$ for the $K^{\pi}=0^{+}$bands in ${ }^{20} \mathrm{Ne}$ from the $2 B M$ calculation.

Experimental Quantity

$\left.\left|<\chi_{\mathbf{K}}\right| \mathbf{Q}_{20}{ }^{\prime}\right|_{\chi_{K}}>\mid$

$\mathrm{K}^{\pi}=\mathrm{O}_{2}{ }^{+11}$

$\mathrm{B}\left(\mathrm{E} 2 \cdot 2^{+} \rightarrow 0^{+}\right)=13.6 \mathrm{e}^{2} \mathrm{fm}^{4}$

8.25

$\mathrm{B}\left(\mathrm{E} 2 \mathrm{~A}^{+} \rightarrow 2^{+}\right)=3.18 \times 10^{-2} \mathrm{e}^{2} \mathrm{fm}^{4}$

0.334

$\mathrm{B}\left(\mathrm{E} 2 \downarrow 6^{+}-4^{+}\right)=27.4 \mathrm{e}^{2} \mathrm{fm}^{4}$

9.33

$\mathrm{B}\left(\mathrm{E} 2+8^{+}-6^{+}\right)=5.97 \times 10^{-2} \mathrm{e}^{2} \mathrm{fm}^{4}$

0.426

$\mathrm{Q}\left(2^{+}\right)=10.63 \mathrm{efm}^{2}$

11.7

$\mathrm{K}^{\pi}=\mathrm{O}_{3}{ }^{+1)}$

$\mathrm{B}\left(\mathrm{E} 2 \cdot 2^{+} \rightarrow 0^{+}\right)=41.4 \mathrm{e}^{2} \mathrm{fm}^{4}$

14.3

$\mathrm{B}\left(\mathrm{E} 2 \mathrm{1}^{+} \mathrm{4}^{+}-2^{+}\right)=30.5 \mathrm{e}^{2} \mathrm{fm}^{4}$

10.3

$\mathrm{B}\left(\mathrm{E} 2 b^{+} 6^{+}-4^{+}\right)=12.3 \mathrm{e}^{2} \mathrm{fm}^{4}$

6.25

$\mathrm{B}\left(\mathrm{E} 2 \mathrm{t} \mathrm{8}^{+} \rightarrow 6^{+}\right)=1.30 \times 10^{-2} \mathrm{e}^{2} \mathrm{fm}^{4}$

0.199

$\mathrm{Q}\left(2^{+}\right)=-8.957 \mathrm{efm}^{2}$

9.89

$\mathrm{K}^{\pi}=\mathrm{O}_{2}^{+2)}$

$\mathrm{B}\left(\mathrm{E} 2\right.$ เ $\left.2^{+}-0^{+}\right)=13.6 \mathrm{e}^{2} \mathrm{fm}^{4}$

8.25

$\mathrm{B}\left(\mathrm{E} 2 \mathrm{t}^{+} \rightarrow 2^{+}\right)=3.18 \times 10^{-2} \mathrm{e}^{2} \mathrm{fm}^{4}$

0.334

$\mathrm{B}\left(\mathrm{E} 216^{+} \rightarrow 4^{+}\right)=27.4 \mathrm{e}^{2} \mathrm{fm}^{4}$

9.33

$\mathrm{B}\left(\mathrm{E} 2 \mathrm{1} 8^{+}-6^{+}\right)=5.97 \times 10^{-2} \mathrm{e}^{2} \mathrm{fm}^{4}$

0.426

$\mathrm{Q}\left(2^{+}\right)=10.63 \mathrm{efm}^{2}$

11.7

$\mathrm{K}^{\pi}=\mathrm{O}_{3}{ }^{+2)}$

$\mathrm{B}\left(\mathrm{E} 2 \downarrow 2^{+} \rightarrow 0^{+}\right)=41.4 \mathrm{e}^{2} \mathrm{fm}^{4}$

$\mathrm{B}\left(\mathrm{E} 2 \mathrm{4}^{+}-2^{+}\right)=30.5 \mathrm{e}^{2} \mathrm{fm}^{4}$

10.33

$\mathrm{B}\left(\mathrm{E} 2{ }^{\prime} 6^{+} \rightarrow 4^{+}\right)=12.3 \mathrm{e}^{2} \mathrm{fm}^{4}$

$\mathrm{B}\left(\mathrm{E} 2+8^{+}-6^{+}\right)=1.30 \times 10^{-2} \mathrm{e}^{2} \mathrm{fm}^{4}$

0.199

$\mathrm{Q}\left(2^{+}\right)=-8.957 \mathrm{efm}^{2}$

9.89

1)Assuming the $\mathrm{O}_{2}^{+}$and $\mathrm{O}_{3}{ }^{+}$bands do not cross.

2) Assuming the $\mathrm{O}_{2}^{+}$and $\mathrm{O}_{3}^{+}$bands do cross. 
included in Figure III-7 and Table III-10 and their in-band E2 transitions are given in Table III-12. The ZBM calculation fits the ground-state band as well as the (sd) calculation did, but the ZBM calculation also fits the $2^{-}$band well. Both shellmodel calculations have trouble with the excited $\mathrm{O}^{+}$bands. Since the $\mathrm{O}_{3}^{+}$band is believed to be an $8 \mathrm{p}-4 \mathrm{~h}$ band, a larger calculation, perhaps allowing up to 4 holes in the p3/2 orbit, may be necessary to adequately explain the structure of these states. Neither calculation gets the $0_{4}^{+}$band, which supports the belief that it is either an $(f p)^{4}$ or $(s d)^{2}(f p)^{2}$ band. The ZBM calculation also seems to get the $1^{-}$ band, which is not well known experimentally, although the calculated energies are off significantly.

\section{III-3. Other Models}

While the previous two models are the only ones which will be used to analyze the data presented here, many other models of nuclear structure have been applied to ${ }^{20} \mathrm{Ne}$. Two of the most commonly used models are the Nilsson model (NI-55, PR-75 gives a good introductory description) and SU3 (HA-68 gives a good introductory description). While neitner of these is used in the analysis of the data, the Nilsson model can aid in an understanding of the structure of the bands in ${ }^{20} \mathrm{Ne}$.

The Nilsson model gives a level diagram similar to the level diagram from the shell model, but with the dependence of the energies of the levels on deformation. The lowest levels are shown in Figure III-8. The structure of the first four $\mathrm{K}^{\pi}=0^{+}$ bands in ${ }^{20} \mathrm{Ne}$ is shown in Figure I-2 and for the negative-parity bands in Figure I3. As was seen in the shell-model calculations, the $\mathrm{O}_{2}^{+}$band is an sd-shell excitation, and the $\mathrm{O}_{3}^{+}$band involves the excitation of particles out of the p-shell. The $\mathrm{O}_{1}^{+}$band is seen to be an excitation of four particles into the fp-shell, which is why neither shell-model calculation was able to explain it. The $2^{-}$band involves the 
TABLE III-12.

$\mathrm{B}(\mathrm{E2})$ 's for transitions for negative parity bands in the ZBM calculation.

\begin{tabular}{|c|c|c|}
\hline$J_{1}{ }^{\pi}$ & $J_{f}{ }^{\pi}$ & $B(E \lambda l)\left(e^{\lambda} f m^{2 \lambda}\right)$ \\
\hline \multicolumn{3}{|c|}{$K^{\pi}=2^{-}$} \\
\hline $3^{-}$ & $2^{-}$ & 72.9 \\
\hline $4^{-}$ & $2^{-}$ & 25.5 \\
\hline $4^{-}$ & $3^{-}$ & 57.0 \\
\hline $5^{-}$ & $3^{-}$ & 32.6 \\
\hline $5^{-}$ & $4^{-}$ & 34.9 \\
\hline $6^{-}$ & $4^{-}$ & 36.9 \\
\hline $6^{-}$ & $5^{-}$ & 18.3 \\
\hline $7^{-}$ & $5^{-}$ & 31.9 \\
\hline $7^{-}$ & $6^{-}$ & 14.4 \\
\hline \multicolumn{3}{|c|}{$K^{\pi}=0^{-11}$} \\
\hline $3^{-}$ & $1^{-}$ & 29.8 \\
\hline $5^{-}$ & $3^{-}$ & 37.9 \\
\hline $7^{-}$ & $5^{-}$ & 51.9 \\
\hline \multicolumn{3}{|c|}{$K^{\pi}=1^{-2)}$} \\
\hline $3^{-}$ & $1^{-}$ & 29.8 \\
\hline $3^{-}$ & $2^{-}$ & 35.3 \\
\hline $4^{-}$ & $2^{-}$ & 33.0 \\
\hline $4^{-}$ & $3^{-}$ & 24.5 \\
\hline $5^{-}$ & $3^{-}$ & 37.9 \\
\hline $5^{-}$ & $4^{-}$ & 11.1 \\
\hline $6^{-}$ & $4^{-}$ & 18.8 \\
\hline $6^{-}$ & $5^{-}$ & 18.3 \\
\hline $7^{-}$ & $5^{-}$ & 17.1 \\
\hline $7^{-}$ & $6^{-}$ & 13.1 \\
\hline $8^{-}$ & $6^{-}$ & 16.6 \\
\hline
\end{tabular}

${ }^{1)}$ Assuming the second negative parity band has $K^{\pi}=0^{-}$.

${ }^{2)}$ Assuming ihe second negative parity band has $\mathrm{K}^{\pi}=1^{-}$. 


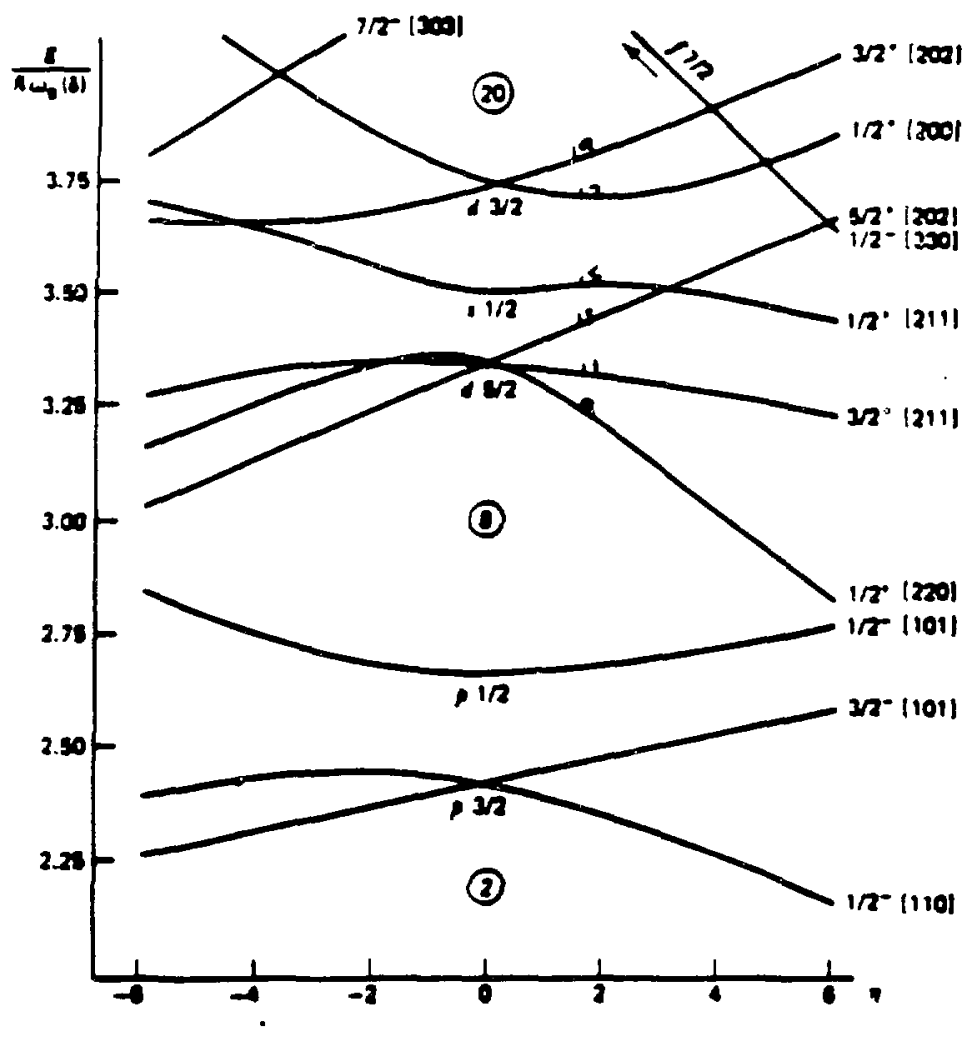

Figure III-8.

Approximate single-particle levels for the Nilsoon Hamiltonian (SH-74), $\eta$ is a meavuse of the deformation of the nucleus. 
excitation of one particle out of the p-shell as in the shell-model calculation, as does the $1^{-}$band, and the $0^{-}$band involves the excitation of one particle in to the fp-shell. Because of the extreme deformity of ${ }^{20} \mathrm{Ne}$ the energies of some of the lowest levels in the fp-shell are dropped so far down into the sd-shell that it becomes energetically favorable for excitations to fill them before filling some sdshell orbitals. The spherical shell-model calculations which did not include the fpshell could not explain the structure of these bands. 


\section{Pi-nucleus Scattering Theory}

As in nuclear structure theory, there are several approaches to scattering theory. Most calculations are done in coordinate space or momentum space; for $\pi$-nucleus scattering calculations there are advantages and disadvantages to both. For inelastic scattering the form of the transition density depends on the nuclear structure model used; bott microscopic and collective transition densities will be used to analyze the data presented here. The first section of this chapter will describe $\pi$-nucleus elastic scattering, in both coordinate and momentum space. The second section will describe inelastic scat-oring, collective transitions in coordinate space and microscopic transitions in momentum space. Two-step or coupled channels calculations will be described at the end of the second section.

\section{IV-1. Elastic Scattering}

The $\pi$-nucleus coordinate-space elastic-scattering calculations were done using the formalism in PIRK (EI-74). The description of Eisenstein and Miller (EI-74) will be followed here. The program PIRK solves the potential scattering problem of a relativistic spin zero particle interacting with a complex optical potential. 
This is done by integrating a Klein-Gordon equation out to a match point and calculating phase shifts by comparing the internal wave function with an external, asymptotic Coulomb wave function.

The Klein-Gordon equation is obtained from the relativistic energy relation

$$
E^{2}-p^{2} c^{2}=m^{2} c^{4}
$$

with the substitution $p=-i k \nabla$. If electromagnetic potentials are included the equation becomes

$$
(E-e \phi)^{2}-\left(p-\frac{e}{c} A\right)^{2} c^{2}=m^{2} c^{4}
$$

where $E$ is the total energy, $p$ is the 3 -momentum, and $m$ is the mass of the pion. $A$ is the electromagnetic vector potential and $\phi$ is the scalar potential. The interaction between the pion and the nucleus is purely electrostatic so $A=0$. The potential $\phi$ is calculated assuming the nucleus is a uniformly charged sphere. The $\pi$-nucleus potential is not completely understood and it is commonly assumed that it transforms like the 4 th component of a 4-vector, like the energy so

$$
E-E-V_{e}-V_{N}
$$

where $V_{N}$ is the nuclear potential and $V_{c}=e \phi$. In addition the terms $2 V_{c} V_{N}$ and $V_{N}^{2}$ are arbitrarily dropped from the equation for $E^{2}$ giving

$$
E^{2} \cdot\left(E-V_{c}\right)^{2}-2 E V_{N}
$$

With these assumptions for tba $\pi$-nucleus interaction, the Klein-Gordon equation is

$$
\left[-\left(m \nabla_{p}\right)^{2}+\frac{c^{2} L^{2}}{r^{2}}+\left(m c^{2}\right)^{2}\right] \psi=\left(E^{2}-2 V_{c} E+V_{c}^{2}-2 E V_{N}\right) \psi
$$

The operator $L^{2}$ is orbital angular momenturn squared and $\nabla_{r}$ is the radial part of 
the gradient. Next it is convenient to make several substitutions and definitions:

$$
\begin{gathered}
\psi=\sum_{l} \frac{u_{l}(r)}{r} i Y_{l, m}(\theta, \phi), \\
U_{c}=\frac{V_{c}}{l c}, \quad U_{N}=\frac{V_{N}}{l c}, \quad \xi=\frac{E}{b c},
\end{gathered}
$$

and

$$
k^{2}=\left(\frac{P}{h}\right)^{2}=\frac{\left(E^{2}-m^{2} c^{4}\right)}{(k)^{2}}
$$

giving for the Klein-Gordon equation

$$
\left(-\nabla_{\mathrm{p}}^{2}+\frac{(l+1)}{r^{2}}\right) \frac{u_{l}}{r} Y_{m}=\left[k^{2}+U_{e}^{2}-2 \xi\left(U_{e}+U_{N}\right)\right] \frac{u_{l}}{r} Y_{t m} .
$$

As mentioned above the $\pi$-nucleus potential is not well known. There are several forms available in the literature for the optical potential $U_{N}$, which will be discussed later in this section. With suitable choices for the constants $A_{1}, A_{2}, A_{3}$, and $A_{4}$, the following form works for most models,

$$
2 \xi U_{N} \psi=\left[A_{1} \rho+A_{2} \nabla \cdot \rho \nabla+A_{3} \nabla^{2} \rho+A_{4} \nabla^{4} \rho\right] \psi .
$$

where $\rho$ is the nuclear matter density, which is assumed to be spherically symmetric. Using this form for the potential, the radial equation for each partial wave becomes

$$
\begin{gathered}
\left(A_{2} \rho-1\right) u_{i}{ }^{\prime}+A_{2} \rho^{\prime} u_{i}^{\prime} \\
=\left[\frac{A_{2} \rho^{\prime}}{r}+k^{2}-2 U_{c} \xi+U_{c}^{2}-A_{3} \nabla_{r}^{2} \rho-A_{4} \nabla_{i}^{4} \rho-A_{1} \rho+\frac{l(l+1)}{r^{2}} \cdot\left(A_{2} \rho-1\right)\right] u_{b}
\end{gathered}
$$

where the primes indicate differentiation with respect to $r$. This equation is integrated out from the origin to the matching radius. At the matching radius the 


$$
-70-
$$

inner wave function, $u_{l}$, is compared with the outer wave function, which is a solution to the Coulomb scattering problem in the absence of $U_{N}$. The phase shift, $\delta_{l}$, is obtained from the relation

$$
\mathrm{S}_{l}=\exp \left(2 \mathrm{i} \delta_{l}\right)=\left(\frac{\mathrm{D}(\mathrm{F}+\mathrm{iG})-\left(\mathrm{F}^{\prime}+\mathrm{iG}\right)}{\left(\mathrm{F}^{\prime}-\mathrm{i} \mathrm{G}^{\prime}\right)-\mathrm{D}(\mathrm{F}-\mathrm{iG})}\right)_{i}
$$

where $F$ and $G$ are the regular and irregular Coulomb wave functions, $S$, is the $S$ matrix element, and $\mathrm{D}$ is the logarithmic derivative of the inner wave function at the match point

$$
\mathrm{D}=\left.\frac{\mathrm{u}_{1}^{\prime}}{\mathrm{u}_{l}}\right|_{\mathrm{r}=\text { matchpoint }}
$$

The differential and reaction cross sections are calculated from the following formulas:

$$
\begin{aligned}
& \frac{\mathrm{d} \sigma}{\mathrm{d} \Omega}=|(\theta)|^{2} \\
& f(\theta)=f_{c}(\theta)+f_{N}(\theta), \\
& \mathrm{f}_{c}(\theta)=-\frac{\eta}{2 \mathrm{p} \sin ^{2} \psi_{2} \theta} \exp \left[2 \mathrm{i}\left(\sigma_{0}-\eta \ln \left(\sin \psi_{2} \theta\right)\right)\right], \\
& \eta=Z_{N} Z_{\pi} \alpha \frac{E}{p} . \\
& f_{N}(\theta)=\frac{1}{i k} \sum_{l}(2 l+1) \exp \left(2 i \sigma_{l}\right) f_{P}(\cos \theta), \\
& \mathrm{f}_{f}=\frac{1 / 2}{2}\left(\exp \left(2 \mathrm{i} \delta_{l}\right) \cdots 1\right)=1 / 2\left(S_{r}-1\right),
\end{aligned}
$$

and

$$
\sigma_{\text {resction }}=\frac{\pi}{k^{2}} \Sigma(2 l+1)\left(1-\left|\mathrm{S}_{\mid}\right|^{2}\right)
$$


where $\sigma_{l}$ is the point charge Coulomb phase shift of the lth partial wave and $\alpha$ is the fine structure constant.

As mentioned above the $\pi$-nucleus optical potential is not well known. The most frequently used potential is the Kisslinger potential (KI-55, KI-74). Including $s$ and $p$ wave interactions, it is

$$
V=-b_{0} k^{2} \rho+b_{1} \nabla \cdot \rho \nabla,
$$

where $\mathrm{k}$ is the pion momentum in the $\pi$-nucleus center of mass frame. The spindependent parts of the $\pi$-nucleus interaction have been left out of the optical potential. The complex coefficients $b_{0}$ and $b_{1}$ are calculated from $\pi$-nucleon phase shifts, $\delta^{\mathrm{L}}{ }_{2 J, 2 \mathrm{~T}}$, by averaging over the number of neutrons and protons in the target. For even-even nuclei they are (BL-84)

$$
b_{0}=\frac{4 \pi \gamma}{k^{3}} \frac{\left(2 \alpha_{31}^{0}+\alpha_{11}^{0}\right)}{3}
$$

and

$$
b_{1}=\frac{4 \pi y}{k^{3}} \frac{\left(4 \alpha_{33}^{1}+2 \alpha_{31}^{1}+2 \alpha_{13}^{1}+\alpha_{11}^{1}\right)}{3}
$$

where

$$
\alpha_{2 T, 2 J}^{L}=\exp \left(i \delta_{2 J, 2 T}^{L}\right) \sin \left(\delta_{2 J}^{L}, 2 T\right)
$$

and

$$
\gamma=\frac{\tilde{\omega}_{\pi} \bar{\omega}_{N}}{\omega_{\pi} \omega_{N}} .
$$

The phase shifts of Rowe, Salomon, and Landau (RO-78) have been used in calculations presented here. The quantity $y$ transforms the $\pi$-nucleon interaction from the $\pi$-nucleon center of mass to the $\pi$-nucleus center of mass, $(\mathrm{CO}-80)$, where $\tilde{\omega}_{\pi}$ 
and $\bar{\omega}_{N}$ are the pion and nucleon energies in the $\pi$-nucleon center-of-mass frame, and $\omega_{\pi}$ and $\omega_{N}$ are their energies in the $\pi$-nucleus center-of-mass frame. The agreement between the calculation and experiment can be improved greatly by evaluating the $\pi$-nucleon t-matrix elements $\approx 28 \mathrm{MeV}$ lower than the $\pi$-nucleus center of mass energy (CO-80).

The $\pi$-nucleus momentum-space elastic-scattering calculations were done using the formalism in PIPIT (EI-76b). The description of Eisenstein and Tabakin (EI-76b) will be followed here. The program PIPIT solves a relativistic Lippmann-Schwinger equation in momentum space to obtain the elastic $\mathrm{T}$-matrix elements. In momentum space the Lippmann-Schwinger equatint takes the form

$$
T^{\prime}\left(\mathbf{k}, \mathbf{k}^{\prime}: \mathbf{k}_{\mathbf{0}}\right)=\mathrm{C}^{\prime}\left(\mathbf{k}, \mathbf{k}^{\prime}\right)+\int \frac{T^{\prime}\left(\mathbf{k}, \mathbf{k}^{\prime \prime} ; \mathbf{k}_{\mathbf{0}}\right) \mathrm{C}^{\prime}\left(\mathbf{k}^{\prime \prime}, \mathbf{k}^{\prime}\right)}{E\left(\mathbf{k}_{\mathbf{0}}\right)-E_{\pi}\left(\mathbf{k}^{\prime \prime}\right)-E_{\mathbf{A}}\left(\mathbf{k}^{\prime \prime}\right)+i \epsilon} \mathrm{d}^{3} \mathbf{k}^{\prime \prime},
$$

where $E\left(k_{0}\right)$ is the total on-shell energy in the $\pi$-nucleus center-of-mass Irame and $E_{\pi}$ and $E_{A}$ are the total energies of the pion and nucleus defined relativistically. te.

$$
E_{\pi}=\left(k_{0}^{2}+m_{\pi}^{2}\right)^{4}
$$

and similarly for $E_{A}$. The quantities $k^{\prime}, k^{\prime \prime}$, and $k_{0}$ are pion momenta in the $\pi$ nucleus center-of-mass frame. The optical potential, $U\left(k, k^{\prime}\right)$ is obtained from the multiple scattering formalism of Kerman, McManus, and Thaler (KMT) and will be discussed later in this section.

The $\pi$-nucleus $T$-matrix in KMT formalism differs from the $T^{\prime}$ defined above

$$
T=\frac{A}{A+1} T^{\prime}
$$

where $A$ is the atomic mass number. The cross section can be written in terms of the reaction matrix, $R^{\prime}$, which is related to $T^{\prime}$ by 


$$
T^{\prime}(\omega)=R^{\prime}(\omega)-\pi i R^{\prime}(\omega) \delta\left(\omega-H_{0}\right) T^{\prime}(\omega)
$$

$\mathrm{R}^{\prime}$ and $\mathrm{U}^{\prime}$ are decomposed using

$$
\mathrm{U}\left(\mathbf{k}, \mathbf{k}^{\prime}\right)=\frac{2}{\pi} \sum_{\mathrm{m} m} \mathrm{U}_{\mathrm{m}_{\mathbf{m}}}\left(\mathbf{k}, \mathrm{k}^{\prime}\right) \mathrm{Y}_{\mathrm{tm}}(\mathbf{k}) \mathrm{Y}_{\mathrm{m}}^{\prime}\left(\mathbf{k}^{\prime}\right)
$$

and

$$
R^{\prime}\left(k, k^{\prime} ; k_{0}\right)=\frac{2}{\pi} \sum_{\text {mo }} R^{\prime}\left(k, k^{\prime}: k_{0}\right) Y_{\text {mo }}(k) Y_{\text {mo }}^{\prime}\left(k^{\prime}\right)
$$

This gives the following integral equation for $R^{\prime}$,

$$
\begin{gathered}
R^{\prime}\left(k, k^{\prime} ; k_{0}\right)= \\
U\left(k, k^{\prime}\right)+\frac{2}{\pi} P \int \frac{R^{\prime}\left(k, k^{\prime \prime} ; k_{0}\right) U\left(k^{\prime \prime}, k^{\prime}\right)}{E_{\pi}\left(k_{0}\right)+E_{A}\left(k^{\prime \prime}\right)-E_{\pi}\left(k^{\prime \prime}\right)-E_{A}\left(k^{\prime \prime}\right)} d^{3} k^{\prime \prime}
\end{gathered}
$$

where $\mathrm{P}$ denotes a principal value integral. This equation is solved for $\mathrm{R}^{\prime}$.

The on-shell $T^{\prime}$, is related to the on-shell $R^{\prime}$, by

$$
\mathrm{T}^{\prime}=\mathrm{R}^{\prime},\left[1-\underline{\mathrm{i}} \mathrm{\rho}(\mathrm{E}) \mathrm{T}^{\prime},\right]
$$

where $\rho(E)$ is a phase space factor

$$
\rho(E)=\frac{2 k_{0} E_{\pi} E_{A}}{\left(E_{\pi}+E_{A}\right)(x)^{2}}
$$

$T_{l}$ and $R_{l}$ are related to the phase shift, $\delta_{l}$ by

$$
\rho(E) T_{F}=-\exp \left(i \delta_{l}\right) \sin \delta_{l}
$$

and

$$
\rho(E) R_{\Gamma}=-\tan \delta_{l}
$$

where $\delta_{l}$ is the phase shift relative to an undistorted outgoing spherical wave $\mathrm{j}\left(\mathrm{k}_{0} \mathrm{r}\right)$. 
Because the Coulomb interaction has a large effect on elastic scattering cross sections it must be included. Using the phase shifts found for the nuclear part of the interaction, the radial part of the wave function, $u(k r)$, can be written

$$
u_{l}-\left[j_{l}+\left[\rho(E) R_{l}\right] n_{l}\right] k r .
$$

The logarithmic derivative, $\xi_{b}$ evaluated at some cutoff radius, $R_{e}$ is

$$
\xi_{l}=\left.\frac{\mathrm{u}_{l}^{\prime}}{u_{l}}\right|_{\mathrm{R}_{e}}
$$

New phase shifts can be defined relative to the outgoing Coulomb wave functions,

$$
\overline{\mathrm{u}}_{l} \approx \mathrm{F}_{\boldsymbol{r}}\left[\rho\left(\mathrm{E}_{i}\right) \mathrm{R}_{l}\right] \mathrm{G}_{l},
$$

where $F$ and $G$ are the regular and irregular Coulomb wave functions. Then

$$
-\rho(E) R=\left.\frac{F^{\prime} \Gamma \xi F_{l}}{\xi G_{\Gamma}-G_{l}^{\prime}}\right|_{r=R_{e}}
$$

The S-matrix is related to $T$ by

$$
S_{h}\left(k_{0}\right)=1-2 i p(E) T\left(k_{0}\right)
$$

and as stated above $T$ is related to $F$ by

$$
\mathrm{T}_{l}=\mathrm{R}_{l}\left[1-\mathrm{i} \mathrm{p}(\mathrm{E}) \mathrm{T}_{l}\right] .
$$

The differential cross section can then be calculated using:

$$
\begin{gathered}
\frac{d \sigma}{d \Omega}=\left.f(\theta)\right|^{p} . \\
f(\theta)=f_{c}(\theta)+f_{A}(\theta), \\
f_{c}(\theta)=-\frac{\eta_{c}}{2 p \sin ^{2} \frac{\theta}{2}} \exp \left[2 i\left(\sigma_{0}-\eta_{c} \ln \left(\sin \psi_{2} \theta\right)\right]\right],
\end{gathered}
$$




$$
\eta_{c}=Z_{A} Z_{\pi} \frac{\alpha E_{\pi} E_{A}}{\left(E_{\pi}+E_{A}\right) p_{\pi}}
$$

and

$$
\mathrm{f}_{\mathrm{A}}(\theta)=\frac{1}{2 \mathrm{i} \mathrm{k}_{0}} \sum_{l}(2 l+1)\left(S_{\Gamma}-1\right) \exp \left(2 \mathrm{i} \sigma_{l}\right) \mathrm{P} /(\cos \theta)
$$

where $\sigma_{l}$ is the point charge Coulomb phase shift and $\alpha$ is the fine structure constant.

As in the coordinate-space calculation the $\pi$-nucleus optical potential is uncertain. In the KMT formalism (KE-59) the first order $\pi$-nucleus optical potential with the factorization approximation is

$$
\tau\left(\mathbf{k}^{\prime}, \mathbf{k}\right)=\frac{A-1}{A}\left[\rho_{p}(\mathbf{q}) t_{\pi p}\left(\mathbf{k}^{\prime}, \mathbf{k} ; \mathbf{k}_{0}\right)+\rho_{\mathrm{a}}(\mathbf{q}) t_{\pi \mathrm{d}}\left(\mathbf{k}^{\prime}, \mathbf{k} ; \mathbf{k}_{0}\right)\right]
$$

where $k$ and $k^{\prime}$ are the relative $\pi$-nucleus momenta and $q=k^{\prime}-\mathbf{k}$ is the iransferred momentum. Since this is a momentum space calculation the nuclear matter density, $\rho(\mathbf{q})$, is the Fourjer transform of the ground state density. The quantities $t_{\pi 0}$ and $t_{\pi p}$ are the $\pi$-neutron and $\pi$-proton scattering matrices, which are constructed from the free $\pi$-nucleon matrices. This form can be made to include the spin-flip part of the interaction.

To accommodate the partial wave decomposition done above, the density and t-matrices are decomposed as follows:

$$
\rho_{1}=\sum_{l_{1}} \rho_{l}{ }^{\prime}\left(k^{\prime}, k\right) P_{l_{1}}\left(k \cdot k^{\prime}\right)
$$

and

$$
t_{\pi 1}\left(k^{\prime}, \mathbf{k} ; k_{0}\right)=\sum_{l_{2}} t_{\pi i}^{l_{2}}\left(k^{\prime}, k ; k_{0}\right) P_{h^{\prime}}\left(k \cdot k^{\prime}\right)
$$


where $i=p, n$. Then

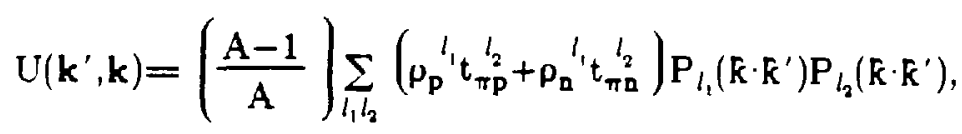

Comparing this to the original decomposition of $U$ and doing some angular momentum algebra the optical potential becomes

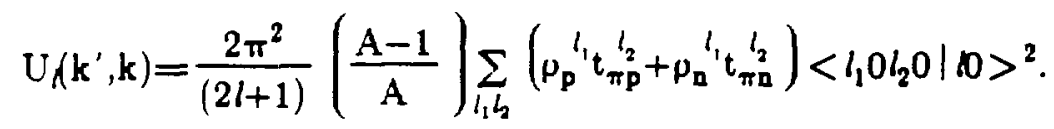

There is another model in the liferature for calculating elastic scattering which has not been used here. The delta-bole model (HI-77, ER-88a) is a conceptually simple model for calculating $\pi$-nucleus elastic scattering near the delta resonance. The delta resonance dominates $\pi$-nucleon scattering for beam energies around $160 \mathrm{MeV}$. For $\pi$-nucleus scattering the delta-hole model assumes that the pion interacts with a nucleon and forms a delta, which propagates through the nucleus. The delta then decays back to a pion and nucleon and the pion is emitted. While this is conceptually very simple, the delta-hole model is more complicated than the optical model. The delta interaction with other nucleons provides two-body corrections to the one-body $\pi-N$ scattering. This calculation also has its uncertainties, in particular bow the delta interacts with the nucleons in the nucleus. This model also agrees reasonably well with experiment. The delta-hole model has also been applied to inelastic scattering and absorption processes (HI$77, \mathrm{LE}-80 c, \mathrm{LE}-82)$. It fits ${ }^{12} \mathrm{C}\left(\pi, \pi^{\prime}\right)$ data very well (LE-82).

\section{IV-2. Inelastic Scattering}

Three types of inelastic scattering calculations have been done in the analysis of the data presented here. The first set used a collective-model transition density 
and did the calculation in coordinate space. In the previous chapter it was shown that ${ }^{20} \mathrm{Ne}$ is well described by a rotational model. The second set used a microscopic transition density and did the calculation in momentum space. The shellmodel calculations described in the previous chapter provided the transition densities used in these calculations. The final set were coupled-channels calculations and used collective-model transition densities in coordinate space.

The collective-model calculations used a modified version of the computer code DWPI (EI-76a). The description of Eisengtein and Miller (El-76a) will be followed here. The cross secticĩ tr a specific excited state is:

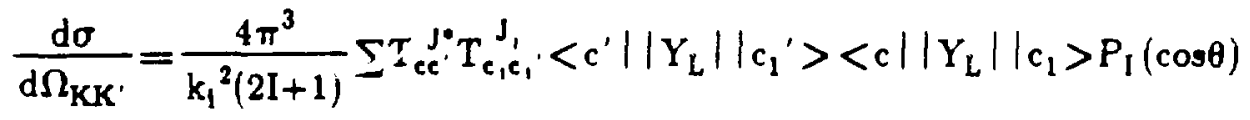

where the sum is over $c, c^{\prime}, c_{1}, c_{1}{ }^{\prime}$, and $L$. The ground state of the target nucleus is described by a set of quantum numbers $K$ and the final state by a set $K^{\prime}$. The center of mass momentum of the incident pion is $k_{1}$ and $I$ is the ground-state angular momentum of the target. $T_{e c}^{J}$ is the transition matrix element for states of total angular momentum I and the channel state $c$ is given by

$$
|c\rangle=|(l \times I) J\rangle
$$

where $l$ is the angular momentum of the pion. Using angular momentum algebra it can be shown (EI-76a) that:

$$
\frac{d c}{d \Omega_{K K^{\prime}}}=\frac{\pi^{2}}{k_{1}{ }^{2}(2 I+1)} \sum_{L} \sigma_{L} P_{L}(\cos \theta)
$$

where

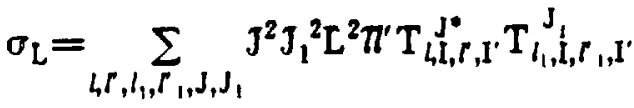

$$
\begin{aligned}
& x<\infty \mathrm{LO}\left|l_{1} 0\right\rangle\left\langle l^{\prime} 0 \mathrm{LO}\right| l_{1} 0>\mathrm{W}\left(\boldsymbol{L I J}_{1} ; l_{1} \mathrm{~J}\right) W\left(l^{\prime} \mathrm{LI}^{\prime} \mathrm{J}_{1}: l^{\prime}{ }_{1} \mathrm{~J}\right),
\end{aligned}
$$


where $X=\sqrt{2 X+1}$ and $I^{\prime}$ is the excited state angular momentum. The $W$ functions are Racah angular momentum coefficients. In the standard distorted-wave impulse approximation (DWLA) treatment the elastic scattering is handled by using distorted waves, which in DWPI are calculated exactly as in PIRK, which was described eariier in this cbapter. The inelastic transition will be treated to first order only. The T-matrix element becomes:

$$
\begin{aligned}
& T_{l, \mathrm{I}, l^{\prime}, \mathrm{I}^{\prime}}^{\mathrm{J}}
\end{aligned}
$$

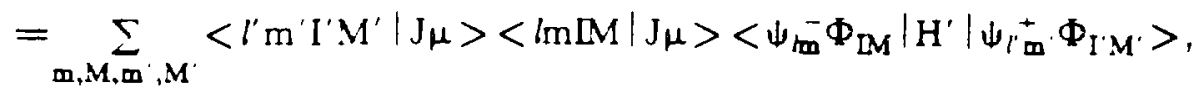

where $m+M=\mu=m^{\prime}+M^{\prime}$.

As was shown in the discussion on elastic scattering the first-order opticalmodel potential is given by

$$
V_{N}=A t_{\pi N} P
$$

which in coordinate space is

$$
V_{. v} \psi=\left[\frac{(b)^{2}}{2 E}\right]\left(A_{1} \rho+A_{2} \nabla \cdot \rho \nabla+A_{3} \nabla^{2} \rho\right) \psi,
$$

where $E$ is the pion energy in the center of mass frame, $\psi$ is the pion wavefunction. and $\rho=\rho(r, c)$ is the deformed ground-state density with c containing the arzular dependence. The term $A_{4} \Gamma^{4} p$ has been ignored. In the collective model the part of the optical potential which causes the nuclear excitation comes from the deformed part of the density, $\delta \rho,(G L-83$, EI-768) and is written

$$
\begin{aligned}
& <\Phi_{I^{\prime} M^{\prime}}|H| \Phi_{M}>=\left\langle I^{\prime} M^{\prime}\left|A t_{\pi n} \delta \rho\right| M\right\rangle \\
& =A t_{\pi n} \sum_{\lambda \mu} F_{\lambda}(r) Y_{\lambda \mu}(\hat{r})<I^{\prime} M^{\prime}\left|\alpha_{\lambda_{\mu}}\right| I M>
\end{aligned}
$$


where

$$
F_{\lambda}=-\left.c \frac{\partial \rho}{\partial r}\right|_{c=c_{0}}
$$

and

$$
\alpha_{\lambda \mu}=\beta_{\lambda} \lambda^{-1}\left[b_{\lambda \mu}+(-)^{\mu} b_{\lambda-\mu}^{+}\right] .
$$

The operators $b_{\lambda_{\mu}}$ and $b_{\lambda_{\mu}}^{\dagger}$ destroy and create a phonon of angular momentum $\lambda$ and projection $\mu$. The pion wavefunctions are

$$
\psi_{\text {tm }}^{ \pm}=i^{\prime} \frac{u_{1} \pm(r)}{r} Y_{h m}(\hat{r})
$$

and are calculated as in PIRK, described above.

Putting the above expressions into the first-order equation for the $T$ matrix it can be shown (EI-76a, ED-71):

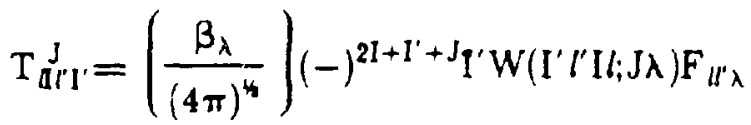

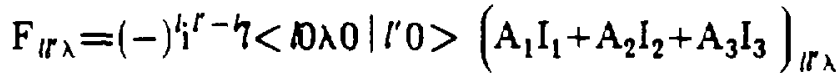

where $I_{1}, I_{2}$, and $I_{3}$ are integrals involving $F_{1}$ and $u_{l}=$. Coulomb excitation can be included in the calculation, but is generally unimportant in pion inelastic scattering.

These calculations give a very good description of transitions to highly collective states where the trangferred angular momentum is greater than one. For transitions where $\Delta /=1$ and $\Delta T=0$ the decay is forbidden by conservation of momentum (DE-73, HA-81), so the integral of the trangition density must be zero. The following transition density can be shown to be appropriate (HA-81): 


$$
\rho^{1}(r)=-\frac{\beta_{1}}{R \sqrt{3}}\left[3 r^{2} \frac{d}{d r}+10 r-\frac{5}{3}<r^{2}>\frac{d}{d r}+\epsilon\left(r \frac{d^{2}}{d r^{2}}+4 \frac{d}{d r}\right)\right] \rho_{0}(r),
$$

where $\rho_{0}$ is the ground-state mass density and

$$
\left.\beta_{1}^{2}=\frac{6 \pi r^{2}}{m A E} R^{2}\left(11<r^{4}>-\frac{25}{3}<r^{2}\right\rangle^{2}-10 \epsilon<r^{2}>\right)^{-1},
$$

where $\beta_{1}$ is a collective parameter for the isoscalar dipole resonance and $R$ is the half-density radius of the mass distribution. The parameter $\epsilon$ is much less than $\left\langle r^{2}\right\rangle$ and can be ignored for $A \geq 20$. Since $A=20$ for ${ }^{20} \mathrm{Ne}$ it is desirable to check this before ignoring $\epsilon$ so the following expression for $\epsilon$ was used (HA-81)

$$
\epsilon=\left(\frac{4}{E_{2}}+\frac{5}{E_{0}}\right) H^{2}(3 \mathrm{~mA})^{-1}
$$

where $E_{2}$ is the excitation energy of the giant quadrupole resonance and $E_{0}$ the excitation energy of the giant monopole resonance.

For $\lambda=0$ transitions the integral of the transition density also must be zero, this time from orthogonality of the $0^{+}$wave functions. Therefore a monopole transition density of the form (AU-71, MO-87)

$$
\rho(r)=3 \rho_{0}(r)+r \frac{d \rho_{0}(r)}{d r}
$$

was used.

The microscopic calculations were done in momentum space using the computer code HLL, which uses the formalism of T.-S. H. Lee et al. (LE-74, I.E-80a, and LE-80b). The degcription of Lee and Lawson (LE-80b) will be followed here. The amplitude for $\pi$-nucleus inelastic scattering is (LE-80a, LE-80b) 


$$
\begin{aligned}
& T_{\theta}\left(k_{0}^{\prime} \Lambda^{\prime}, k_{0} \Lambda\right)=\sum_{L M L^{\prime} M^{\prime} J T}\left(L L^{\prime} T J_{f}\right)^{4}(-1)^{J_{P}-M_{r}+M+1+1} \\
& \times\left(\begin{array}{ccc}
J & J_{l} & J_{f} \\
M_{f}-M_{1} & M_{1} & -M_{f}
\end{array}\right)\left(\begin{array}{ccc}
L & L^{\prime} & J \\
-M & M^{\prime} & M_{1}-M_{f}
\end{array}\right)\left(\begin{array}{ccc}
1 & 1 & T \\
\Lambda & -\Lambda^{\prime} & \Lambda_{l}-\Lambda_{f}
\end{array}\right)
\end{aligned}
$$

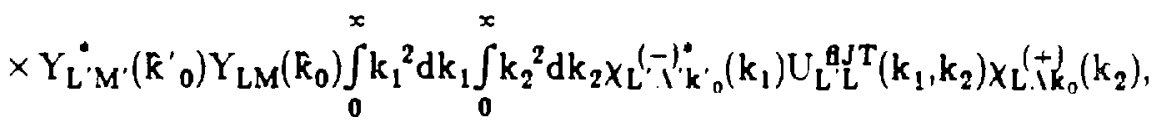

where the $k^{\prime} s$ are the $\pi$-nucleus relative momenta in the center-of-mass frame, $k^{\prime} 0$ and $\mathrm{k}_{0}$ are unit vectors in the direction of the outgoing and incoming pions respectively, $\Lambda$ and $\Lambda^{\prime}$ are the initial and final pion isospins, the spin and isospin quantum numbers $\left|J_{l} M_{1} T_{l} \Lambda_{l}\right\rangle$ and $\left|J_{P} M_{f} T_{P} \Lambda_{f}\right\rangle$ denote the initial and final nucleas states, and the parentheses are $3 \mathrm{j}$ coefficients. The distorted waves $X_{L}\left( \pm k_{0}\right)(k)$ are calculated in PIPIT described above.

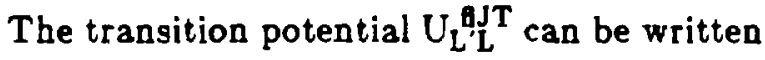

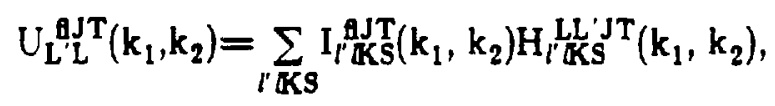

where $\mathrm{H}$ is determined by only the $\pi$-nucleon dynamics,

$$
\begin{aligned}
& \mathrm{H}_{r^{\prime} \mathrm{KK}^{\prime} \mathrm{J} S}^{\mathrm{L}^{\prime} \mathrm{T}}\left(\mathrm{k}_{1}, \mathrm{k}_{2}\right)=\mathrm{i}^{r-1}(\mathrm{~K})^{\prime \prime} \eta^{\prime}\left(\begin{array}{l}
l^{\prime \prime K} \\
000
\end{array}\right)
\end{aligned}
$$

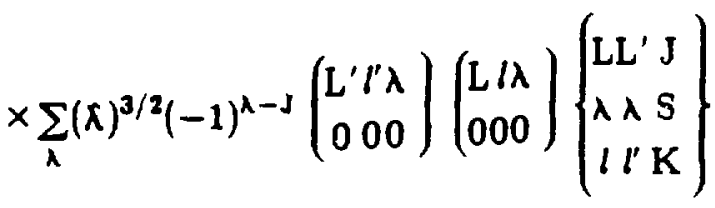

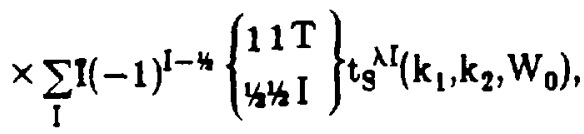

where the small curly trackets denote a $6 \mathrm{j}$, the large curly brackets a $9 \mathrm{j}$, and $t_{g}^{\lambda I}\left(k_{1}, k_{2}, W_{0}\right)$ is the $\lambda$-th partial-wave $\pi$-nucleon amplitude for isospin $I=1 / 2$ or I=3/2 with $S=0$ or $S=1$, and is calculated in PIPIT using the model of 
Londergan, McVoy, and Moniz (LO-74).

All the nuclear structure information is included in

$$
I_{l ' K S}^{\text {AJT }}\left(k_{1}, k_{2}\right)=\int_{0}^{x} j_{l}\left(k_{1} r\right) F_{K S}^{\text {AJT }}(r) j \lambda\left(k_{2} r\right) r^{2} d r
$$

where $\mathrm{j}(\mathrm{kr})$ are spherical Bessel functions and

$$
\begin{aligned}
& F_{\mathrm{KS}}^{\text {gJT }}(r)=(-1)^{T_{r}-\Lambda}\left(T_{f}\right)^{H}\left(\begin{array}{ccc}
T & T_{l} & T_{f} \\
\Lambda_{f}-\Lambda_{1} & \Lambda_{l} & -\Lambda_{f}
\end{array}\right) \\
& \left.\times \sum_{\alpha \beta}\left(4 \pi j_{\alpha}\right)^{h /}<J_{f} T_{f} \||| b_{\alpha}^{\dagger} \times h_{\beta}^{\dagger}\right]_{J T} \||| J_{1} T_{l}> \\
& x<\alpha \mid\left\|\left[Y_{K}(\hat{r}) \times \sigma_{S}\right]_{J}\right\| \beta>R_{n_{a} b_{b}}(r) R_{\left.\mathbf{D}_{\beta}\right\}_{\beta}}(\mathrm{r}),
\end{aligned}
$$

where $\sigma_{S}=1$ when $S=0$ and $\sigma_{S}=\sigma$ when $S=1, b_{\alpha}^{\dagger}$ and $h_{\alpha}^{\dagger}$ create a particle and a hole respectively in the shell-model state $(\mathrm{n} / \mathrm{j})_{\alpha}$, and $\mathrm{R}_{\mathrm{n}_{\alpha} L_{a}}(\mathrm{r})$ is the radial wavefunction of the shell-model state. All of the nuclear structure information is contained in the reduced matrix element $\left.\left\langle\mathrm{J}_{\mathrm{f}} \mathrm{T}_{\mathrm{f}}|||| \mathrm{b}_{\alpha}^{\dagger} \times \mathrm{h}_{\beta}^{\dagger}\right]_{J \mathrm{~T}} \||| \mathrm{J}_{1} \mathrm{~T}_{\mathrm{l}}\right\rangle$ which is provided by a nuclear structure calculation.

For most shell-model calculations to reproduce electromagnetic transition rates it is necessary to use an effective charge for the proton, $1+\delta_{p}$, and for the neutron, $\delta_{n}$. This is necessary because a truncation of the model space is necessary for the calculation to be computationally tractable. In general all but a few orbits are excluded and such exotic things as $\Delta$ 's in the nucleus and meson exchange currents are left out. Instead of dealing with the bare operator $\left(\delta_{p}=0, \delta_{n}=0\right)$ most shell-model calculations use 


$$
\begin{aligned}
T_{K_{\mu}} & =\sum_{i}\left[\frac{e\left(1+\delta_{p}\right)}{2}\left(1-\tau_{z}(i)\right)+\frac{e \delta_{n}}{2}\left(1+\tau_{z}(i)\right)\right] \Gamma_{1}{ }^{K} Y_{K_{v}}\left(\hat{r}_{l}\right) \\
& =\sum_{i}\left[\frac{e\left(1+\delta_{p}+\delta n\right)}{2}-\frac{e\left(1+\delta_{p}-\delta_{n}\right)}{2} \tau_{z}(i)\right] \Gamma_{l}{ }^{K} Y_{K_{1}}\left(\hat{r}_{l}\right) .
\end{aligned}
$$

This is exactly the operator in the pion transition density above when $\sigma_{9}=1$. Therefore, if an effective charge is required for a shell-model calculation to fit the electromagnetic data a similar enhancement factor should be necessary to fit the pion inelastic-scattering data.

The coupled-channels calculations used the computer code NEWCHOP (MO01 ), which is a modified version of the code CHOPIN (RO-01), which in turn is the computer code CHUCK (KU-O1) modified to do relativistic pion scattering. The descriptions of Comfort (Co-01) and Kunz (Ku-01) for CHUCK3 will be followed here, with appropriate modifications for pion scattering.

The total wave function for the scattering system is taken to be

$$
\Psi^{J M}=\sum_{c J_{e} J_{e}} x_{J_{e} l_{e}}^{\rho}\left(k_{e} ; r_{c}\right)\left[Y_{l_{r} j_{e}}{ }^{c} \Phi_{I_{r}}{ }^{c}\right]_{J}^{M},
$$

where $c$ denotes the channel. The function $\Phi$ is the intrinsic wave function of the target, $Y$ is the coupling of the relative angular momentum with the intrinsic wave function of the projectile, and $x$ is the channel wave function for relative motion. This ave function satisfies the Klein-Gordon equation

$$
\left[(E-e \phi)^{2}-\left(p-\frac{e}{c} A\right)^{2} c^{2}\right] \Psi^{J M}=m^{2} c^{4} \Psi^{J M}
$$

Making the same approximations as were made above for PIRK and DWPI one gets for the Klein-Gordon equation: 


$$
\left[-\left(m \nabla_{r}\right)^{2}+\frac{c^{2} L^{2}}{r^{2}}+\left(m c^{2}\right)^{2}\right] \Psi^{J M}=\left(E^{2}-2 V_{C} E+V_{C}^{2}-2 E V_{N}\right) \Psi^{J M}
$$

where $V_{C}$ is the Coulomb potential and $V_{N}$ is the nuclear potential. The coupled equations for $x$ are

$$
\begin{aligned}
& {\left[-\left(r \frac{d^{2}}{d r_{c}^{2}}\right)^{2}+\frac{(h)^{2} l_{c}\left(l_{c}+1\right)}{r_{c}^{2}}+\left(m c^{2}\right)^{2}-E^{2}+2 V_{C} E-V_{C}^{2}+2 E V_{N e c}\right]} \\
& \times \chi_{j_{j_{c} l_{c}}^{J}}^{J}\left(k_{e} ; r_{c}\right)=\sum_{e^{\prime}}-2 E V_{N e c} \cdot \chi_{j_{c} \cdot l_{c}}^{J}\left(k_{e^{\prime} ; r_{c^{\prime}}}\right),
\end{aligned}
$$

where $V_{\text {Nee }}$ is the distorting nuclear potential seen by the incident, intermediate, and outgoing pions and $\mathrm{V}_{\text {Nec' }}$ is the coupling between channels. Both of these potentials are the same as those used in DWPI and described above. The coupled equations are integrated out to a matching radius.

The channel wave functions, $x$, are solved subject to the boundary condition

$$
\begin{aligned}
& \lim _{r \rightarrow x} x_{J_{e} l_{r}}^{J}\left(k_{e} ; r_{e}\right)
\end{aligned}
$$

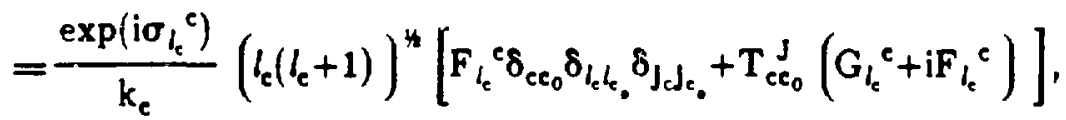

where $c_{0}$ refers to the incident (elastic) channel and $F$ and $G$ are the regular and irregular Coulomb wave functions. The cross section is

$$
\begin{aligned}
& \frac{d \sigma_{c}}{d \Omega}=\frac{1}{2 I_{e_{0}}+1}-\frac{1}{2 S_{c_{0}}+1}
\end{aligned}
$$

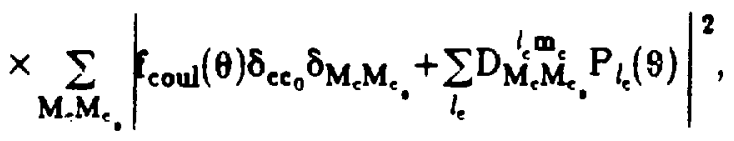

where

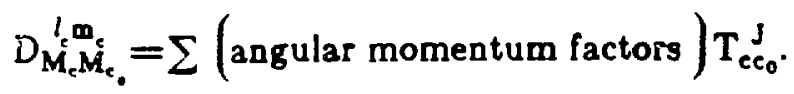


The data presented here have been analyzed using all of the above methods which were appropriate for a given state. The collective calculations should fit the strong transitions very well, in particular the $2_{1}{ }^{+}$, the $3_{1, \overline{2}}$, and the $4_{1}{ }^{+}$transitions. Because spin-flip is relatively weak in pion scattering, and not included in those calculations, unnatural-parity states cannot be fitted by the collective calculations. Because the pion is a strongly interacting particle the decay of a state to the ground state can be very weak, but the corresponding excitation in pion scattering can still be relatively strong, for example if a transition density has a node in the interior of the transition density the pion will never see the node. The surface of the nucleus is the most important region of the transition density in pion scattering near the delta resonance. Also if a state has a strong coupling to either the $2_{1}{ }^{+}$, the $3_{1, \overline{2}}$, or the $4_{1}^{+}$, which couple strongly to the ground state, then the state can be excited by a transition through one of those states. 


\section{Analysis and Results}

This experiment was proposed to look for two-step contributions to pion inelastic scattering. We chose ${ }^{20} \mathrm{Ne}$ for this study because the structure of many of its states is well known both experimentally and theoretically, as discussed in Chapters I and III. There are several states in ${ }^{20} \mathrm{Ne}$ for which two-step contributions could be important. Angular distributions were messured at $180 \mathrm{MeV}$ with both $\pi^{+}$and $\pi^{-}$beams and at $120 \mathrm{MeV}$ with $\pi^{+}$. The data were taken on the EPICS channel at LAMPF and replayed using $Q$, the standard data acquisition and replay system at LAMPF, as was described in Chapter Il. This chapter will discuss the analysis of the spectra and the extracted angular distributions, in particular those for which coupled-channels contributions appear to be important. Results for peaks at high excitation are given in Appendix I.

\section{V-1. Spectra}

Spectra were measured from $12^{\circ}$ to $90^{\circ}$ with $180-\mathrm{MeV}^{+}$and $\pi^{-}$beams, and from $15^{\circ}$ to $90^{\circ}$ with $120-\mathrm{MeV} \pi^{+}$. The data were normalized to hydrogen, using the phase shifts of Rowe, Salomon, and Landau (RO-78). A fitted spectrum at $30^{\circ}$ 
for a $180-\mathrm{MeV} \pi^{+}$incident beam is shown in Figures V-1 through V-5. The data have peaks up to $17.5 \mathrm{MeV}$ excitation. Because a gas target was used, the gas cell. whose composition is given in Table $\mathrm{V}-1$, contaminates the spectra with impurity peaks. Several empty-target spectra were measured to determine the contribution of the gas ceil to the spectra; the $30^{\circ} 180-\mathrm{MeV} \pi^{+}$spectrum is shown in Figure V-6. The only significant impurity peaks come from ${ }^{60} \mathrm{Fe}$, the elastic and $4.5-\mathrm{MeV} 3^{-}$. Oakley (OA-87) has studied pion inelastic scattering on ${ }^{60} \mathrm{Fe}$, as well as ${ }^{54} \mathrm{Fe}$ and ${ }^{52} \mathrm{Cr}$, so the contributions of the strongest impurities are well understood. Inelastic scattering bas also been done on the nickel isotopes (ZU-01). No evidence of peaks from excited states of other neon isotopes is present in the spectra.

The spectra were fitted using the computer code NEWFIT (MO-02). The large elastic peak was used to obtain a peak shape at each angle. For states with a large natural width, $\Gamma$, the peak shape was convoluted with a Lorentzian of width $\Gamma$. Below $9 \mathrm{MeV}$ only known states of ${ }^{20} \mathrm{Ne}$ were used in the fits and their energies were fixed at their values in the latest compilation (AJ-87). The broad $2^{+}$state seen in ${ }^{18} \mathrm{O}(\alpha, \alpha)$ at $\approx 8.8 \mathrm{MeV}$ is seen at $9.00 \pm 0.18 \mathrm{MeV}$ with a width of $\approx 800$ $\mathrm{keV}$. Between 9 and $12.5 \mathrm{MeV}$ the compilation was used as a guide in determining where states should be and what their widths are, but the energies and widths were not required to agree exactly with the compilation. Because the resolution in this experiment was $180 \mathrm{keV}$, above $12.5 \mathrm{MeV}$ ine density of states is so high that the compilation was no longer a useful guide and peaks were inserted where necessary to fit the spectra. A total of 57 peaks, not counting impurity peaks, were used in fitting the spectra; the peaks seen are listed in Table V-2 with states from the compilation (AJ-87) which they may correspond to. Angular distributions have been extracted and analyzed for all 57 peaks. 


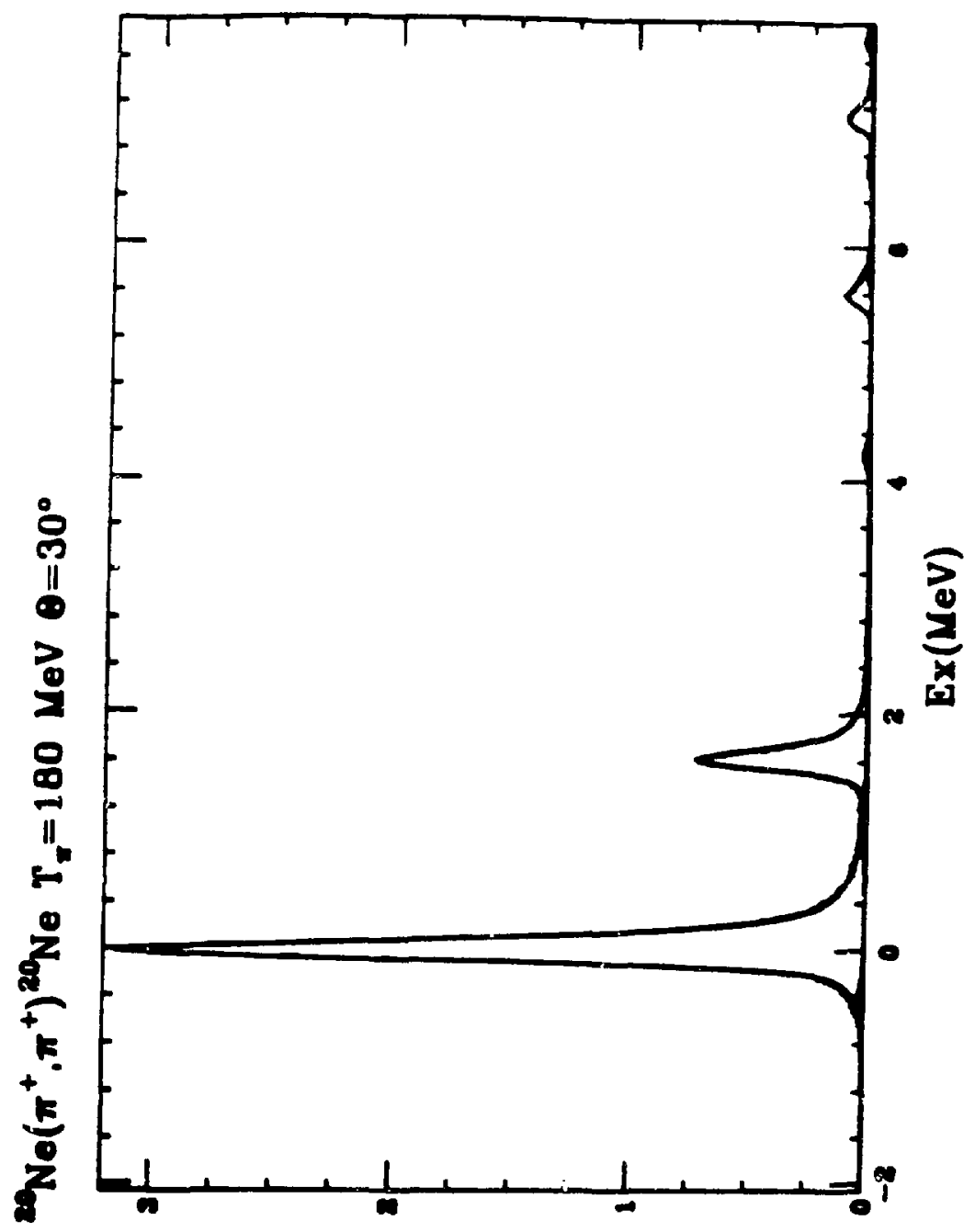

$(\Lambda \cdot \pi 20 \cdot 0 \cdot 20 / q m)$ IPUP/OP

Figure V-1.

Fitted miniog man spectrum for ${ }^{20} \mathrm{Ne}\left(\pi^{+}, \pi^{+\prime}\right)$ at $30^{\circ}$ with $\mathrm{T}_{\pi}=180 \mathrm{MeV}$ from -2 to $8 \mathrm{MeV}$ excitation. 


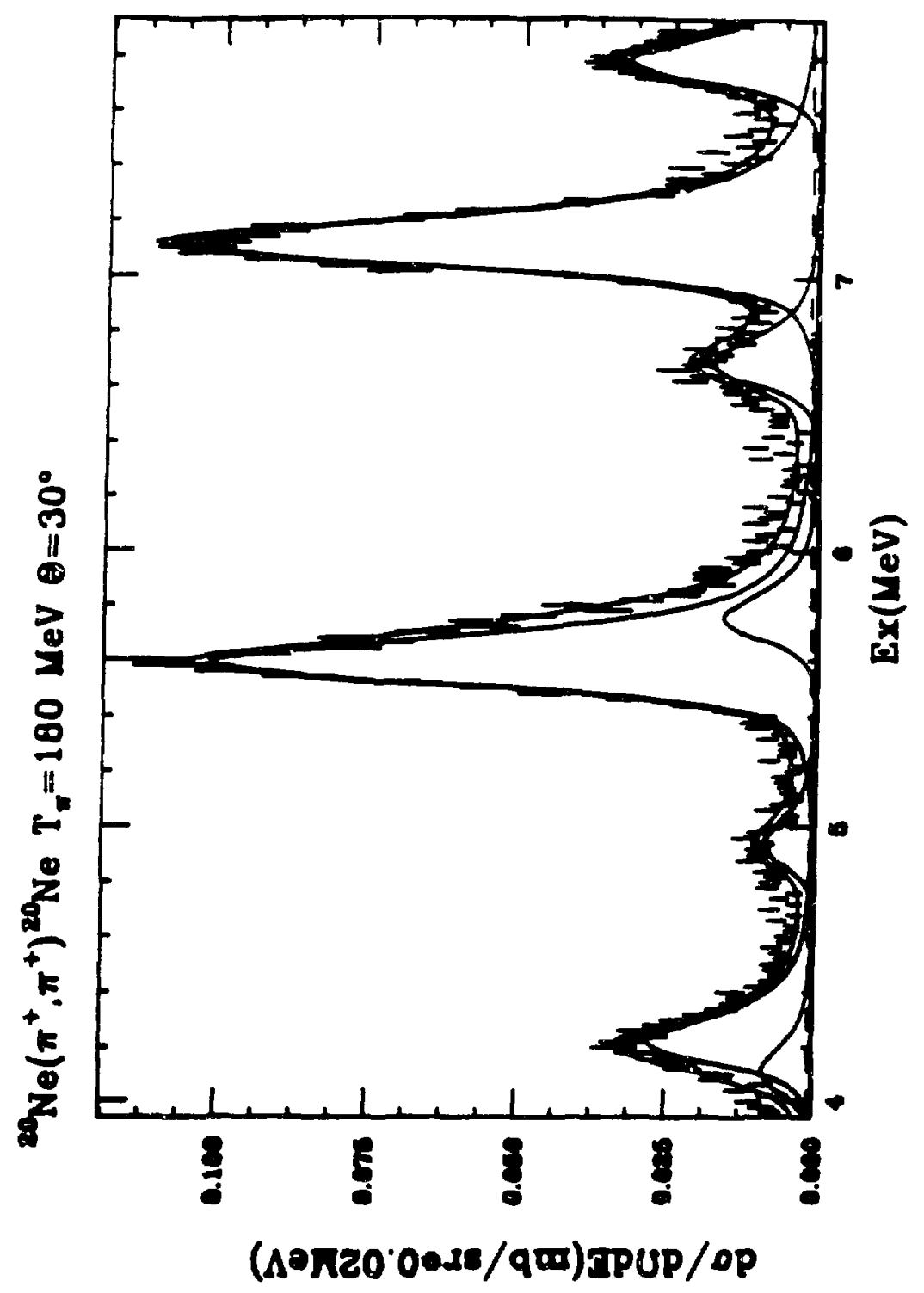

Figure V-2.

Same as Figure V-1 but for 4 to $8 \mathrm{MeV}$ excitation. 


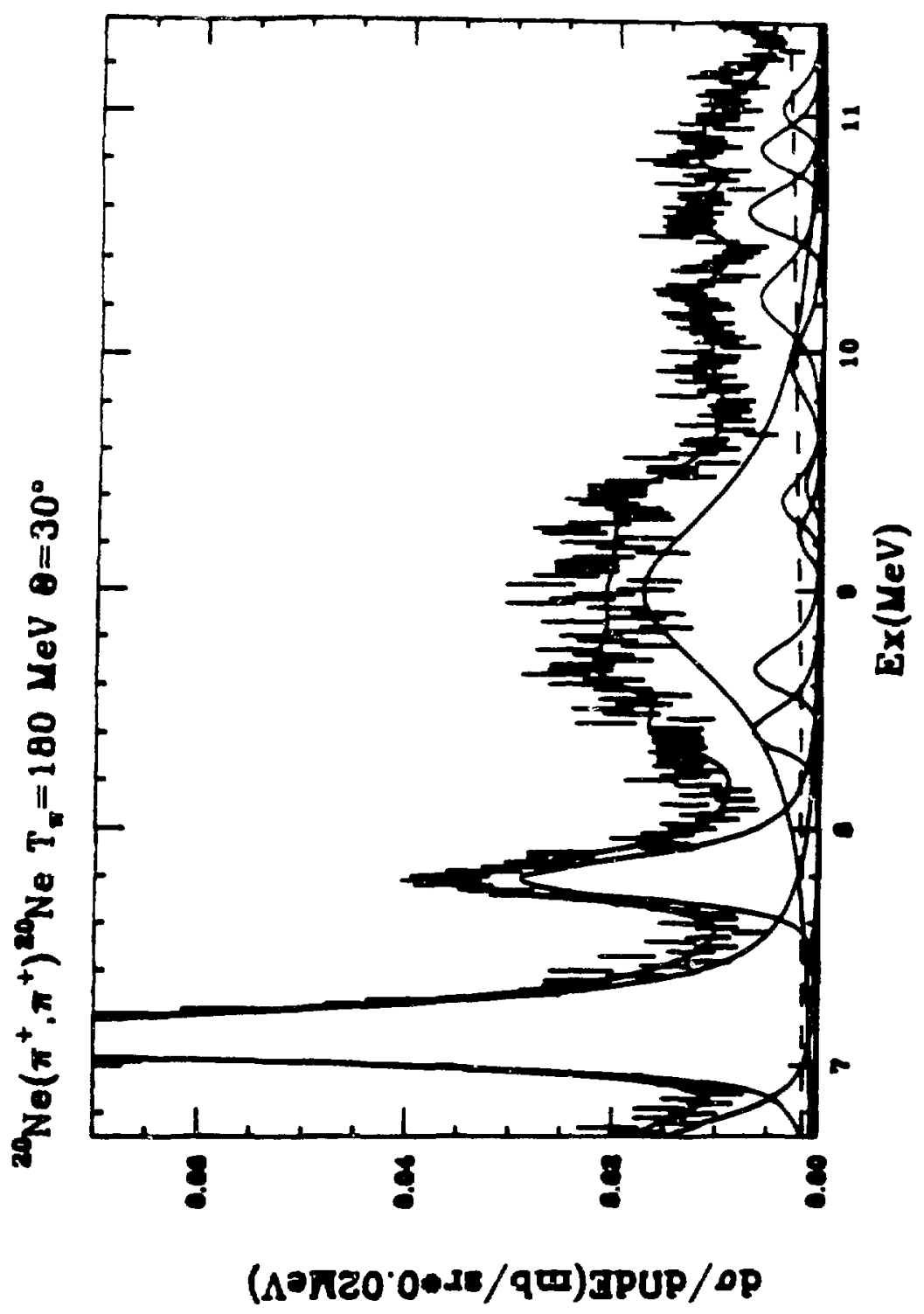

Figure V-3.

Same as Figure V-1 but for 7 to $11.5 \mathrm{MeV}$ excitation. 


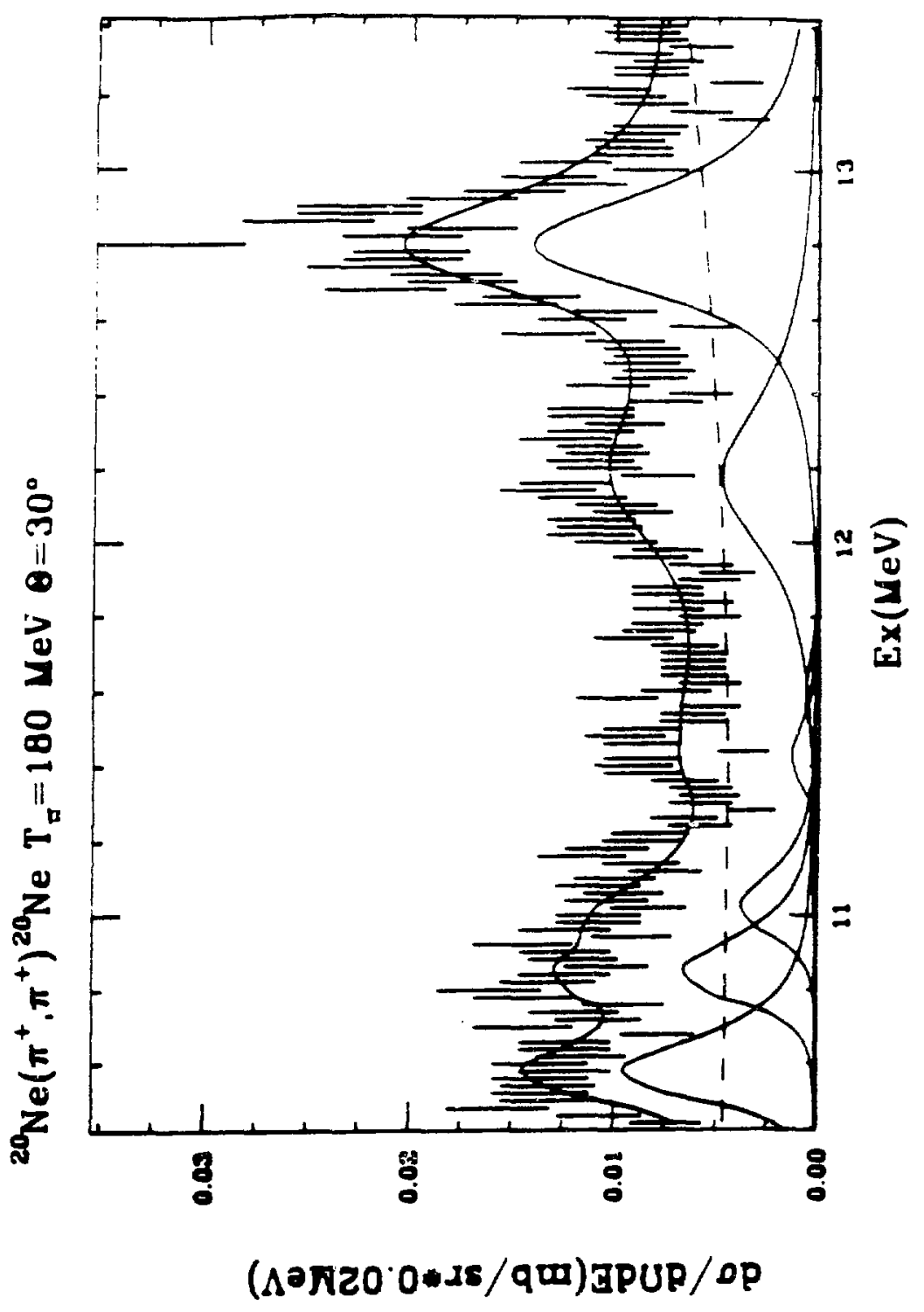

Figure V-4.

Same as Figure V-i but for 10.5 to $13.5 \mathrm{MeV}$ excitation. 


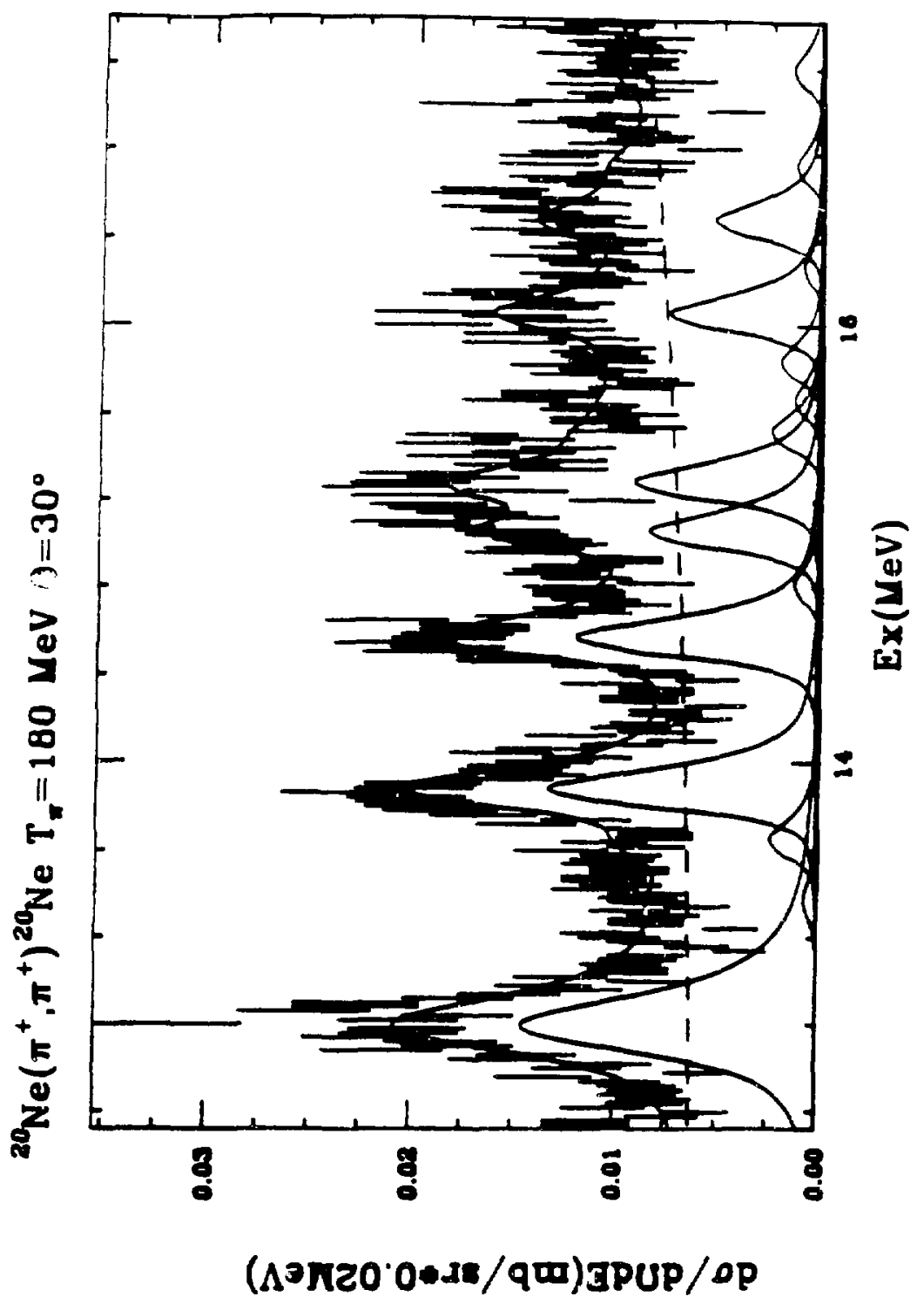

Figure V-5.

Same as Figure V-1 but for 12.5 to $17.5 \mathrm{MeV}$ excitation. 
.93 -

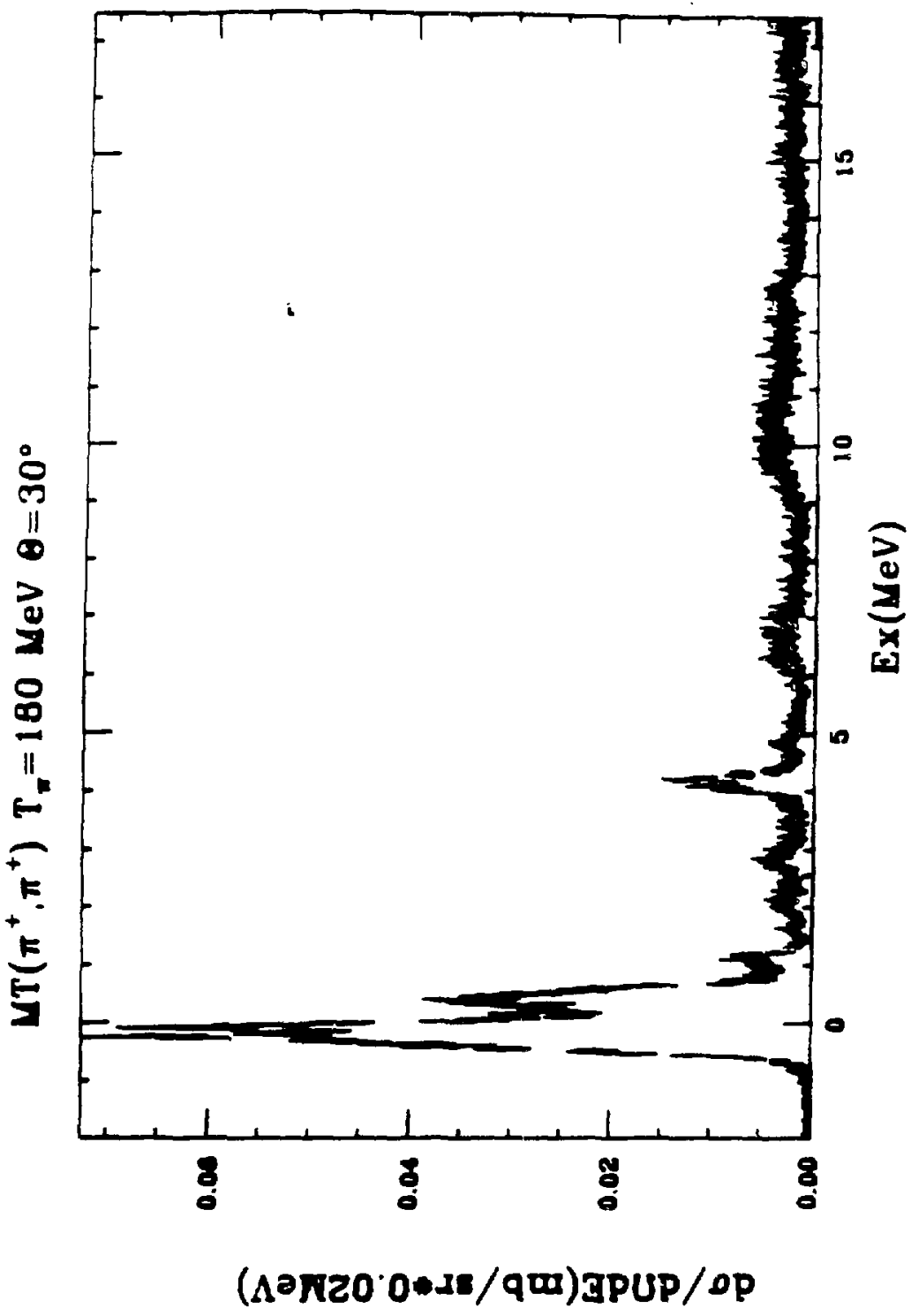

Figure V-6.

Empty target misoing mas histogram for $180-\mathrm{MeV} \pi^{+}$at $30^{\circ}$. 
$-94-$

TABLE V-1.

EPICS gas cell composition.

$\begin{array}{ll}\text { Isotope } & \text { Fraction } \\ { }^{50} \mathrm{Fe} & 64.4 \% \\ { }^{62} \mathrm{Cr} & 15.3 \% \\ { }^{58} \mathrm{Ni} & 6.1 \% \\ { }^{54} \mathrm{Fe} & 4.1 \% \\ { }^{60} \mathrm{Ni} & 2.3 \% \\ { }^{56} \mathrm{Mn} & 1.7 \% \\ { }^{53} \mathrm{Cr} & 1.7 \% \\ { }^{67} \mathrm{Fe} & 1.5 \%\end{array}$


TABLE V-2.

\begin{tabular}{|c|c|c|c|c|}
\hline \multicolumn{5}{|c|}{ Peaks in ${ }^{20} \mathrm{Ne}$} \\
\hline$E_{x}(\mathrm{MeV})^{l}$ & $\Gamma(\mathrm{keV})^{1}$ & $E_{x}(\mathrm{MeV})^{2)}$ & $\Gamma(\mathrm{keV})^{2}$ & $\mathrm{~J}^{\pi} ; \mathrm{T}^{2)}$ \\
\hline 0.00 & & 0.0 & & $0^{+}: 0$ \\
\hline 1.63 & & 1.63 & & $2^{+} ; 0$ \\
\hline 4.25 & & 4.25 & & $4^{+} ; 0$ \\
\hline 4.97 & & 4.97 & & $2^{-} ; 0$ \\
\hline 5.62 & & 5.62 & & $3^{-} ; 0$ \\
\hline 5.79 & & 5.79 & $(2.8 \pm 0.3) \times 10^{-2}$ & $1^{-} ; 0$ \\
\hline \multirow[t]{2}{*}{6.73} & & 6.73 & $19.0 \pm 0.9$ & $0^{+} ; 0$ \\
\hline & & 7.00 & & $4^{-} ; 0$ \\
\hline \multirow[t]{2}{*}{7.16} & & 7.16 & $8.2 \pm 0.3$ & $3^{-} ; 0$ \\
\hline & & 7.19 & $3.4 \pm 0.2$ & $0^{+} ; 0$ \\
\hline 7.42 & & 7.42 & $15.1 \pm 0.7$ & $2^{+} ; 0$ \\
\hline 7.83 & & 7.83 & 2 & $2^{+} ; 0$ \\
\hline \multirow[t]{2}{*}{8.45} & & 8.45 & $0.013 \pm 0.004$ & $5^{-} ; 0$ \\
\hline & & $=8.7$ & $>800$ & $0^{+} ; 0$ \\
\hline 8.71 & & 8.71 & $2.1 \pm 0.8$ & $1^{-} ; 0$ \\
\hline \multirow[t]{3}{*}{8.78} & & 8.78 & $0.11 \pm 0.02$ & $6^{+} ; 0$ \\
\hline & & 8.82 & $<1$ & $\left(5^{-}\right) ; 0$ \\
\hline & & 8.85 & 19 & $1^{-} ; 0$ \\
\hline 9.00 & 800 & $\approx 8.8$ & $>800$ & $2^{+} ; 0$ \\
\hline 9.03 & & 9.03 & 3 & $4^{+} ; 0$ \\
\hline 9.12 & & 9.12 & 3.2 & $3^{-} ; 0$ \\
\hline 9.28 & & 9.32 & & $\left(2^{-}\right) ; 0$ \\
\hline 9.45 & & 9.49 & $29 \pm 15$ & $2^{+} ; 0$ \\
\hline \multirow[t]{2}{*}{9.88} & & 9.87 & & $3^{+} ; 0$ \\
\hline & & 9.94 & & $\left(1^{+} ; 0\right) ; 0$ \\
\hline 9.99 & 155 & 9.99 & $155 \pm 30$ & $4^{+} ; 0$ \\
\hline \multirow[t]{2}{*}{10.26} & 145 & 10.26 & $145 \pm 40$ & $5^{-} ; 0$ \\
\hline & & 10.27 & $\leq 0.3$ & $2^{+} ; 1$ \\
\hline \multirow[t]{3}{*}{10.46} & 80 & 10.41 & 80 & $3^{-} ; 0$ \\
\hline & & 10.55 & 16 & $4^{+} ; 0$ \\
\hline & & 10.58 & 24 & $2^{+} ; 0$ \\
\hline \multirow[t]{2}{*}{10.63} & & 10.61 & & $6^{-} ; 0$ \\
\hline & & 10.69 & & $4^{-}, 3^{+} ; 0$ \\
\hline
\end{tabular}




$$
-96 \text { - }
$$

TABLE V-2.

\begin{tabular}{|c|c|c|c|c|}
\hline \multicolumn{5}{|c|}{ Peaks in ${ }^{20} \mathrm{Ne}$} \\
\hline $\mathrm{E}_{\mathbf{x}}(\mathrm{MeV})^{1}$ & $\Gamma(\mathrm{keV})^{1}$ & $E_{x}(\mathrm{MeV})^{2)}$ & $\Gamma(\mathrm{keV})^{2}$ & $\mathrm{~J}^{\pi} ; \mathrm{T}^{2 l}$ \\
\hline \multirow[t]{4}{*}{10.79} & 350 & 10.80 & 350 & $4^{+} ; 0$ \\
\hline & & 10.84 & 13 & $2^{+} ; 0$ \\
\hline & & 10.84 & 45 & $3^{-} ; 0$ \\
\hline & & 10.88 & & $3^{+} ; 1$ \\
\hline \multirow[t]{2}{*}{10.90} & & 10.92 & 580 & $3^{+} ; 0$ \\
\hline & & 11.02 & 24 & $4^{+} ; 0$ \\
\hline 11.08 & & 11.09 & $\leq 0.5$ & $4^{+} ; 1$ \\
\hline \multirow[t]{3}{*}{11.22} & & 11.24 & 175 & $1^{-} ; 0$ \\
\hline & & 11.26 & & $1^{+} ; 1$ \\
\hline & & 11.27 & $\leq 0.3$ & $1^{-} ; 1$ \\
\hline \multirow[t]{2}{*}{11.48} & & 11.53 & & $3^{+}, 4^{-} ; 0$ \\
\hline & & 11.60 & & $2^{-} ; 1$ \\
\hline 11.64 & & 11.65 & & $\left(3^{+}\right) ; 0$ \\
\hline \multirow[t]{2}{*}{11.83} & & 11.89 & 46 & $2^{+} ; 0$ \\
\hline & & 11.93 & $0.44 \pm 0.15$ & $4^{+} ; 0$ \\
\hline 12.03 & & 12.14 & & $6^{+} ; 0$ \\
\hline \multirow[t]{3}{*}{12.22} & 350 & 12.22 & $<1$ & $2^{+} ; 1$ \\
\hline & & 12.25 & $155 \pm 15$ & $4^{+} ; 0$ \\
\hline & & 12.26 & $<1$ & $3^{-} ; 1$ \\
\hline 12.38 & & 12.33 & $390 \pm 50$ & $2^{+} ; 0$ \\
\hline \multirow[t]{2}{*}{12.58} & & 12.58 & $72 \pm 9$ & $6^{+} ; 0$ \\
\hline & & 12.74 & $61 \pm 12$ & $\left(2^{+}\right) ; 0$ \\
\hline \multirow[t]{3}{*}{12.85} & 150 & 12.84 & $30 \pm 5$ & $1^{-} ; 0$ \\
\hline & & 12.96 & $38 \pm 1$ & $2^{+} ; 0$ \\
\hline & & 13.05 & $18 \pm 3$ & $4^{+} ; 0$ \\
\hline \multirow[t]{2}{*}{13.08} & & 13.10 & $102 \pm 5$ & $6^{+} ; 0$ \\
\hline & & 13.34 & $(8 \pm 3) \times 10^{-2}$ & $7^{-} ; 0$ \\
\hline \multirow[t]{2}{*}{13.43} & & 13.43 & $49 \pm 7$ & $\left(5^{-}\right) ; 0$ \\
\hline & & 13.53 & $61 \pm 8$ & $2^{+} ; 0$ \\
\hline \multirow[t]{3}{*}{13.57} & & 13.57 & $12 \pm 5$ & $2^{+} ; 0$ \\
\hline & & 13.59 & $9 \pm 1$ & $2^{+}$ \\
\hline & & 13.68 & $11 \pm 2$ & $5^{-}$ \\
\hline 13.71 & & 13.74 & $7.7 \pm 0.5$ & $1^{+}$ \\
\hline
\end{tabular}


TABLE V-2.

\begin{tabular}{|c|c|c|c|c|}
\hline \multicolumn{5}{|c|}{ Peaks in ${ }^{20} \mathrm{Ne}$. } \\
\hline$E_{x}(\mathrm{MeV})^{1}$ & $\Gamma(\mathrm{keV})^{1}$ & $\mathrm{E}_{\mathrm{x}}(\mathrm{MeV})^{2)}$ & $\Gamma(\mathrm{keV})^{2}$ & $\mathrm{~J}^{\pi} ; \mathrm{T}^{21}$ \\
\hline & & 13.87 & $\approx 70$ & $1^{-} ; 0$ \\
\hline \multirow[t]{2}{*}{13.94} & & 13.91 & $74 \pm 10$ & $2^{+} ; 0$ \\
\hline & & 14.06 & $\approx 140$ & $2^{+}$ \\
\hline \multirow[t]{4}{*}{14.14} & & 14.12 & $42 \pm 6$ & $2^{+} ; 0$ \\
\hline & & 14.13 & $4.7 \pm 0.7$ & $2^{-}$ \\
\hline & & 14.15 & $11.8 \pm 1.0$ & $2^{-}$ \\
\hline & & 14.30 & $60 \pm 13$ & $\left(6^{+}\right) ; 0$ \\
\hline 14.34 & & 14.31 & $117 \pm 8$ & $6^{+} ; 0$ \\
\hline 14.44 & & 14.54 & $\approx 15$ & $5^{-} ; 0$ \\
\hline \multirow[t]{2}{*}{14.64} & & 14.78 & $110 \pm 20$ & $\left(1^{-}\right)$ \\
\hline & & 14.32 & $117 \pm 13$ & $5^{-} ; 0$ \\
\hline
\end{tabular}

14.88

15.13

$15.05 \quad 66 \pm 20 \quad 2^{+} ; 0$

$15.14 \quad \approx 60 \quad\left(2^{+}\right) ; 0$

15.36

15.17

$230 \pm 25$

$5^{-} ; 0$

15.33

$34 \pm 10$

$4^{+} ; 0$

15.59

15.74

15.91

16.01

100

$\left(2^{+} ; 1\right)$

16.13

16.35

16.57

16.51

$24 \pm 4$

$6^{+} ; 0$

16.56

$90 \pm 30$

$5^{-} ; 0$

16.58

$92 \pm 8$

$7^{-} ; 0$

16.63

$\left(7^{-}\right)$

16.82

16.98

17.13

17.16

$26 \pm 5$

$5^{-} ; 0$

17.27

17.21

$225 \pm 30$

$4^{+} ; 0$

1.) Present work.

2.) Data from AJ-87. Above $10 \mathrm{MeV}$ only relevant states have been included. 


\section{V-2. Ground-state band}

The ground-state $\mathrm{K}^{\pi}=0^{+}$band contains three of the five strongest peaks in the spectra: the elastic, the $1.63-\mathrm{MeV}^{+}$, and the $4.25-\mathrm{MeV}^{+}$. The $8.78-\mathrm{MeV}^{+}$ is also present. The elastic scattering data have been analyzed by W. M. Amos (AM-88). The data are shown in Figure V-7. The curves shown are collectivemodel calculations which model the neutron and proton density of the ground state as a two-parameter Fermi function:

$$
\rho_{\mathrm{n}, \mathrm{p}}(r)=\rho_{0}\left[1+\exp \left(\frac{r-c_{\mathrm{n}, \mathrm{p}}}{a_{\mathrm{n}, \mathrm{p}}}\right)\right]^{-1}
$$

with $c_{\mathrm{a}, \mathrm{p}}=2.805 \mathrm{fm}$ and $\mathrm{a}_{\mathrm{n}, \mathrm{p}}=0.571 \mathrm{fm}(\mathrm{DE}-87)$. The co-ordinate-space calculations were done with the computer code DWPI (EI-76a) and the momentum-space calculations with PIPIT (EI-76b). The curves are absolutely normalized. The agreement is good, although not perfect. The agreement could probably be improved by adding $\rho^{2}$ terms to the $\pi$-nucleus potential (ER-88a,LI-83). The agreement can also be improved by varying $c_{n, p}$ and $a_{n, p}$, but these changes were found to have little effect on the inelastic-scattering calculations. For all inelasticscattering calculations presented here the incident and outgoing pion waves were calculated using the formalism in these codes, as was described in (hapter 4.

The angular distributions for the $2^{+}, 4^{+}$, and $6^{+}$states are sbown in Figures V-8 and V-9 with collective-model, co-ordinate-space calculations from the code DWPI. The curves have been normalized by requiring $M=M_{n}=M_{p}$, where

$$
M_{p}=Z \beta_{p} \int_{0}^{\infty} r^{\prime} \rho_{t r, p} r^{2} d r
$$

and similarly for $M_{n}$, and varying $M$ to fit the $180-\mathrm{MeV} \pi^{+}$and $\pi^{-}$data. The results of this fit are given in Table V-3. 


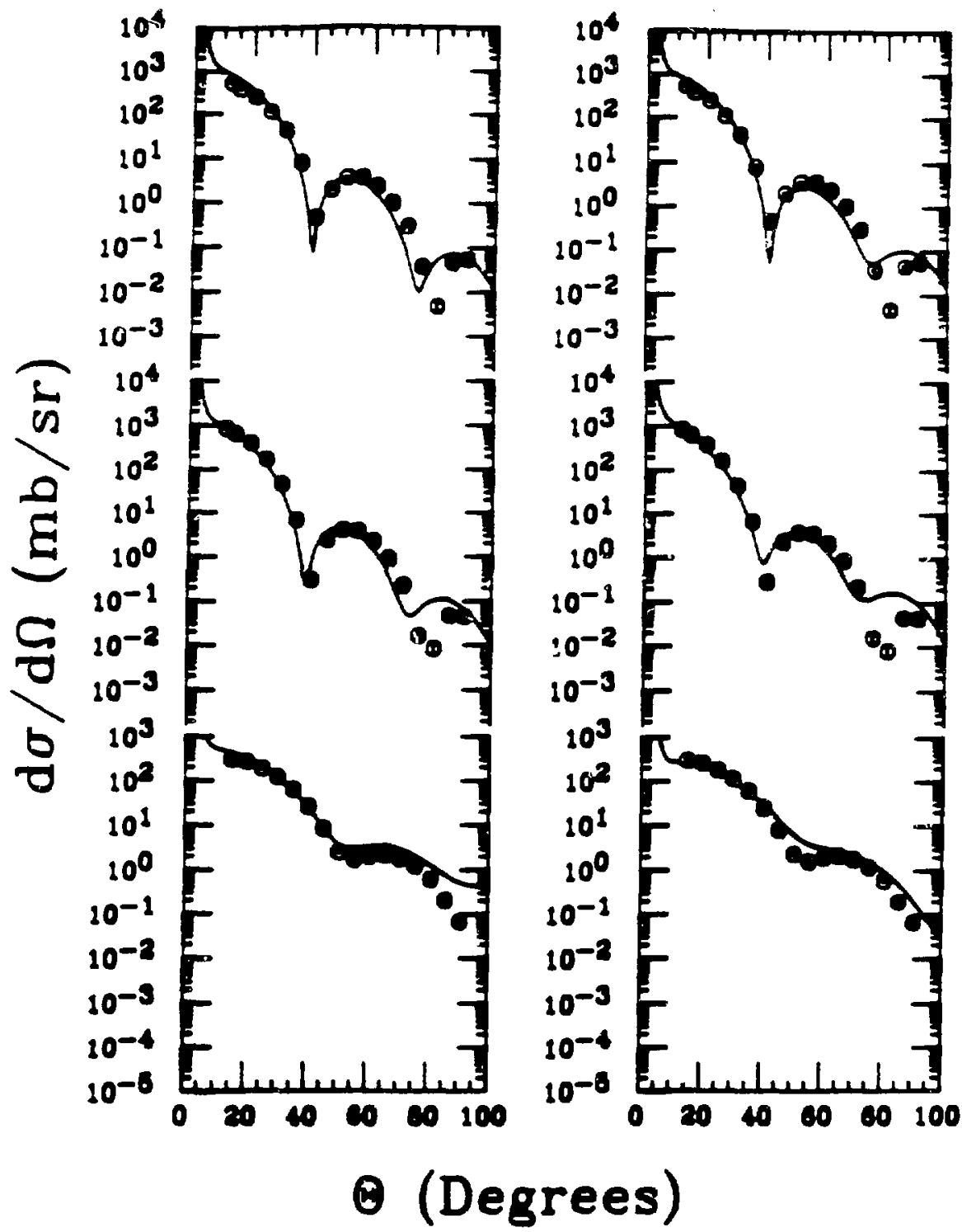

Figure V.7.

Angular distributions for the elastic peak. The top graph in each column is $180-\mathrm{MeV} \pi^{+}$, the middle $180-\mathrm{MeV} \pi^{-}$, and the bottom $120-\mathrm{MeV} \pi^{+}$. The curves in the left column are co-ordinate-space calculations. The curves in the right column are momentum-space calculations. 


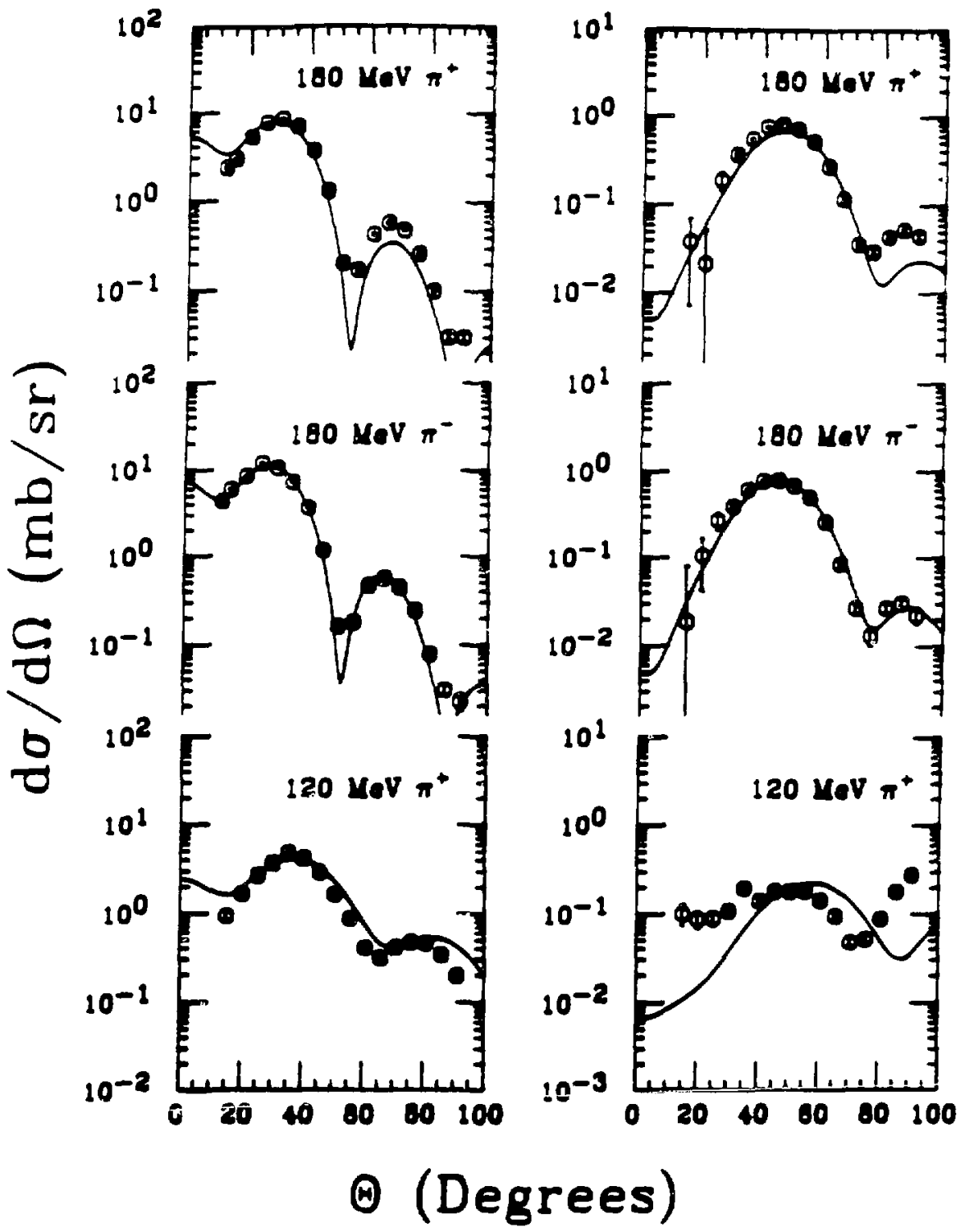

Figure V-8.

Angular distributions for the $1.63-\mathrm{MeV}^{+}$(left column) and the $4.25-\mathrm{MeV}_{4}^{+}$ (right column). The top rraph in each column is $180-\mathrm{MeV} \pi^{+}$, the middle $180-\mathrm{MeV} \pi^{-}$, and the bottom $120 \mathrm{MeV} \pi^{+}$. The curves are DWIA calculations usins a collective-model transition density (DWPI calculations). 


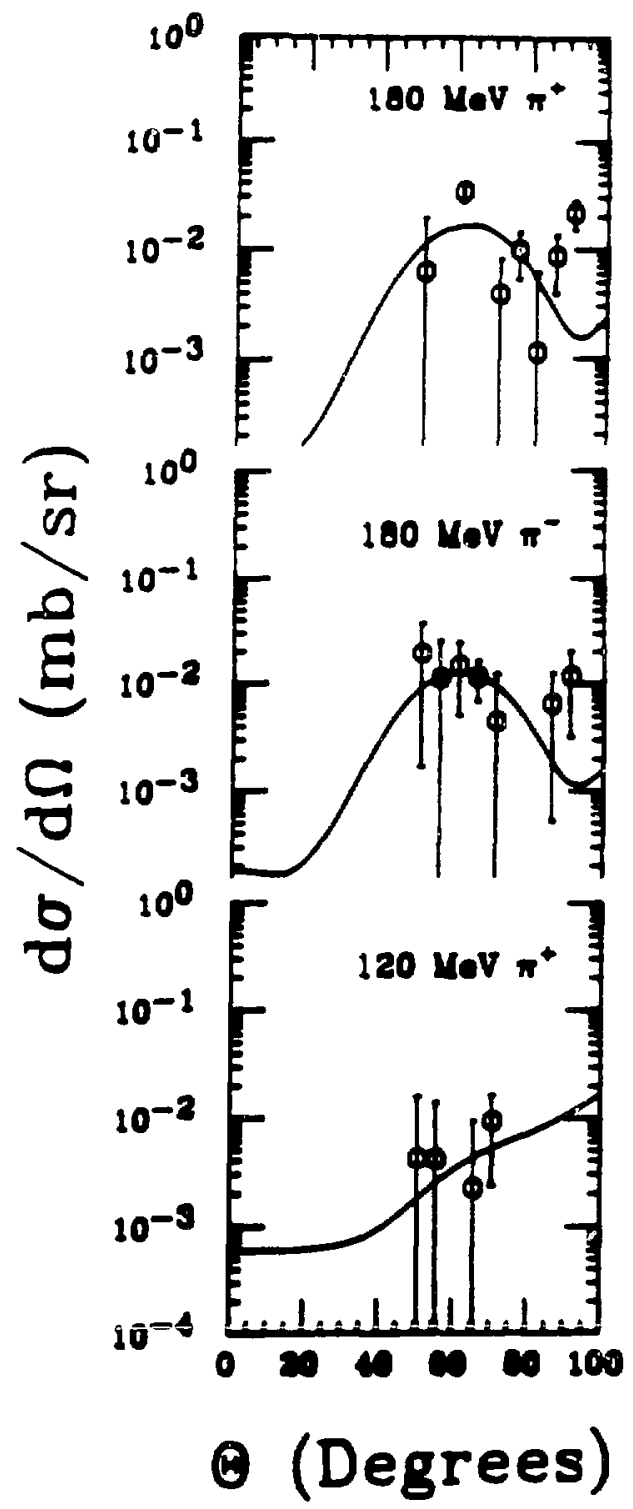

Figure V-9.

Angular distributions for the $8.78-\mathrm{MeV}^{+}$. The top graph is $180 \mathrm{MeV} \pi^{+}$, the middle $180-\mathrm{MeV} \pi^{-}$, and the bottom $120-\mathrm{MeV} \pi^{+}$. The curven are DWPI calculations. 
TABLE V-3.

Ground-state transition strengths extracted with DWLA calculations.

$\begin{array}{llll}\text { Energy } & l^{\prime} & \mathrm{M}\left(\mathrm{e} f \mathrm{~m}^{\prime}\right)^{2)} & \mathrm{B}(\mathrm{E} l \mathrm{t})\left(\mathrm{e}^{2} \mathrm{fm}^{2 l}\right) \\ 1.63 & 2 & 1.797(0.005) \times 10^{1} & 3.23 \times 10^{2} \\ 4.25 & 4 & 2.060(0.015) \times 10^{2} & 4.24 \times 10^{4} \\ 5.62 & 3 & 5.06(0.03) \times 10^{1} & 2.56 \times 10^{3} \\ 7.16 & 3 & 4.57(0.03) \times 10^{1} & 2.09 \times 10^{3} \\ 7.42 & 2 & 1.69(0.13) \times 10^{0} & 2.85 \times 10^{0} \\ 7.83 & 2 & 4.08(0.06) \times 10^{0} & 1.66 \times 10^{1} \\ 8.45 & 2 & 2.09(009) \times 10^{0} & 4.35 \times 10^{0} \\ & 5 & 2.19(0.19) \times 10^{2} & 4.78 \times 10^{4} \\ 8.78 & 6 & 1.5(0.3) \times 10^{3} & 2.16 \times 10^{\circ} \\ 9.00 & 2 & 6.40(0.16) \times 10^{0} & 4.09 \times 10^{1} \\ 9.03 & 4 & 9.9(0.3) \times 10^{1} & 9.83 \times 10^{3} \\ 9.99 & 4 & 7.1(0.4) \times 10^{1} & 5.05 \times 10^{3} \\ 10.26 & 5 & 6.17(0.17) \times 10^{2} & 3.80 \times 10^{6} \\ 10.46 & 3 & 6.4(1.2) \times 10^{0} & 4.13 \times 10^{1} \\ 10.79 & 4 & 7.7(0.9) \times 10^{1} & 6.00 \times 10^{3}\end{array}$

1.) $l$ is the angular momentum transfer used to fit the data. If more than one $l$ is listed then several is were used to fit the data separately and the $M$ listed is for each fit.

2.) All M's were obtained by fitting $180 \mathrm{MeV} \pi^{+}$and $\pi^{-}$data simultaneously, with the constraint $M=M_{n}=M_{p}$. Results for peaks with $l=0$ or 1 contributions are in Table V-5. The number in parentheses is the statiatical error of the fit. 
It is somewhat surprising to see the $6^{+}$, since in the sd-shell the largest singleparticle transition allowed is $d-d$ which gives $l=4$, i.e. going to a $4^{+}$state. For this reason and because the E2 transitions between these states are known to be strong, (AJ-87), coupled-channels calculations have been done using the code NEWCHOP (MO-01). The couplings uged are shown in Figure V-10 and their strengths are given in Table V-4. The results of these calculations are shown in Figure V-11. The calculations are absolutely normalized, but the strength of the $2^{+}-6^{+}$transition is not known and was assumed to be equal to the $0^{+}-4^{+}$strength. No E6 was used in the $6^{+}$calculation, showing that the state can be entirely populated by coupled-channels reaction routes.

Some of the details of the coupled-channels calculations are shown in Figure V-12. The top graph in each column shows the CCLA calculation (solid curve) and the DWIA calculation (dashed curve). The main effect of the twastep route on the $4^{+}$calculation appears to be a shift in the position of the minimum. Destructive interference between the direct $\left(0^{+}-4^{+}\right)$and the two-step $\left(0^{+}-2^{+}-4^{+}\right.$, labelled 2P1 after the intermediate state) calculations is necessary to it the $4^{+}$data, both in shape and normalization. In the second graph in each column the relative contribution of each route is jhüru. The solid curve is the sum of all routes uged, i.e. the entire calculation. For the $4^{+}$, left column, the dashed curve is the $2 \mathrm{P} 1$ route and the dot-dashed curve is the direct route. The strength of the direct route was varied until the entire calculation reproduced the magnitude of the data. The strengths of the E2 transitions were fixed at the known values in the compilation (AJ-87). The $6^{+}$calculation is dominated by the $4 \mathrm{P} 1$ route $\left(0^{+}-4^{+}-6^{+}\right)$, shown by the alternating long and short dashed line in the middle graph. The dashed line is the $2 \mathrm{P} 1$ route $\left(0^{+}-2^{+}-6^{+}\right)$. The streagth of the $2^{+}-6^{+}$transition was assumed to be the same as the strength of the $0^{+}-4^{+}$transition. The $0^{+}-2^{+}-4^{+}-6^{+}$route is 


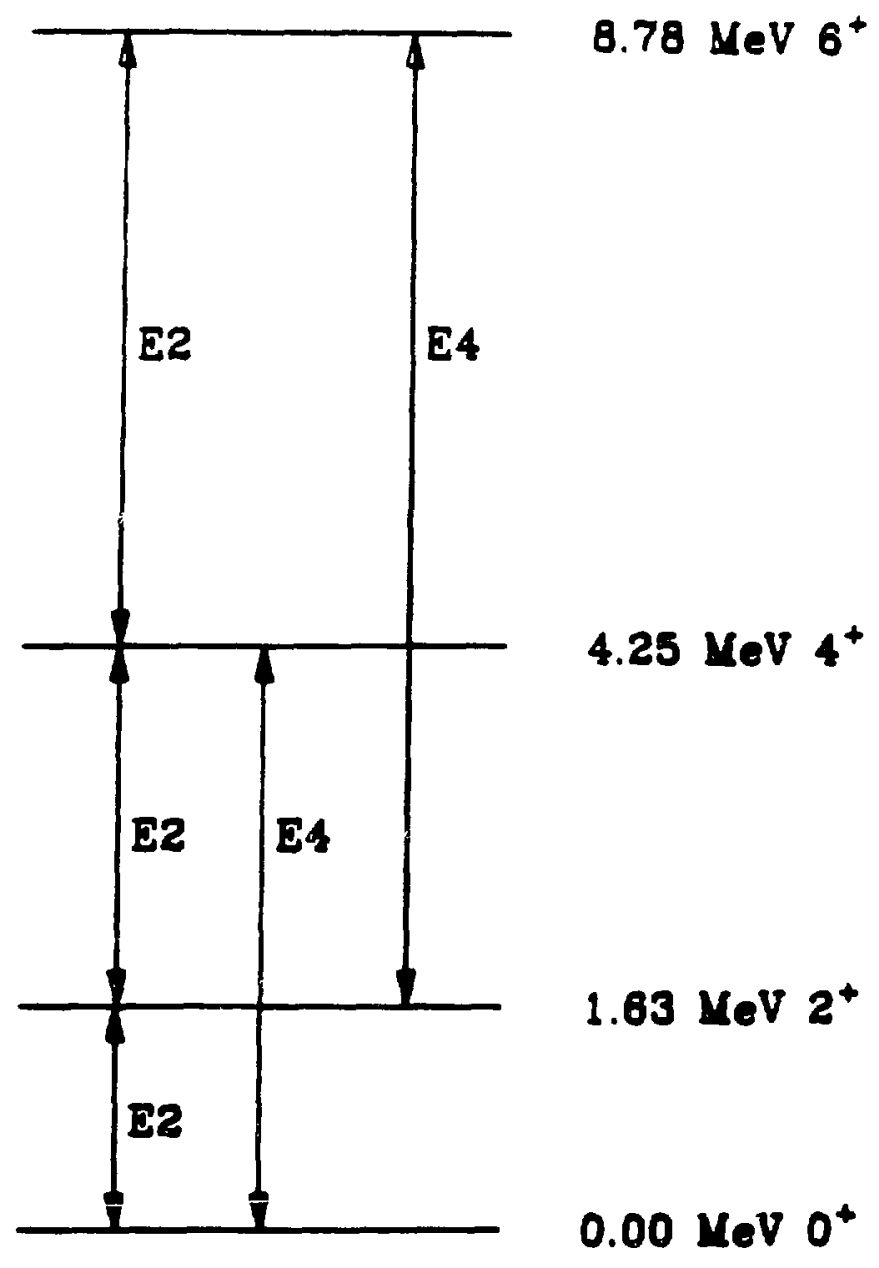

Figure V-10.

Couplings used in the CCLA calculations for states in the $\mathrm{K}^{\pi}=\mathrm{O}_{1}^{+}$band. 
TABLE V-4.

Transition strengths used in CCLA calculations.

\begin{tabular}{lllll}
$E_{1}$ & $E_{f}$ & $l$ & $M\left(\mathrm{em}^{l}\right)$ & $B(E ; ; i-f)\left(\mathrm{e}^{2} \mathrm{fm}^{21}\right)$ \\
0.0 & 1.63 & 2 & $18.0^{1}$ & 323 \\
1.63 & 4.25 & 2 & $25.4^{1}$ & 129 \\
0.0 & 4.25 & 4 & 278 & $7.73 \times 10^{4}$ \\
\hline 0.0 & 1.63 & 2 & $18.0^{1}$ & 323 \\
1.63 & 5.79 & 3 & $45.7^{2}$ & 418 \\
0.0 & 7.16 & 3 & $45.7^{1}$ & $2.09 \times 10^{3}$ \\
7.16 & 5.79 & 2 & $10.8^{1}$ & 17 \\
0.0 & 5.79 & 1 & $16.6^{3}$ & 276 \\
\hline 0.0 & 1.63 & 2 & $18.0^{1}$ & 323 \\
1.63 & 6.73 & 2 & $3.46^{1}$ & 2.4 \\
0.0 & 6.73 & 0 & $5.27^{3}$ & \\
\hline 0.0 & 1.63 & 2 & $18.0^{1}$ & 323
\end{tabular}

$\begin{array}{llllll}1.63 & 7.42 & 2 & 5.24^{1} & 5.5\end{array}$

\begin{tabular}{lllll}
\hline 0.0 & 5.62 & 3 & $50.6^{1}$ & $2.56 \times 10^{3}$ \\
5.62 & 8.45 & 2 & $31.1^{1}$ & 140
\end{tabular}

$\begin{array}{lllll}0.0 & 8.45 & 5 & 384 & 1.48 \times 10^{6}\end{array}$

\begin{tabular}{lllll}
\hline 0.0 & 1.63 & 2 & $18.0^{1}$ & 323 \\
1.63 & 8.71 & 3 & 98.2 & $1.93 \times 10^{3}$ \\
\hline 0.0 & 1.63 & 2 & $18.0^{1}$ & 323
\end{tabular}

$\begin{array}{lllll}1.63 & 4.25 & 2 & 25.4^{1} & 129\end{array}$

$\begin{array}{lllll}4.25 & 8.78 & 2 & 4.65^{1} & 2.4\end{array}$

$\begin{array}{llllll}0.0 & 4.25 & 4 & 278^{1} & 7.73 \times 10^{4}\end{array}$

$\begin{array}{llllll}1.63 & 8.78 & 4 & 278^{4} & 1.55 \times 10^{4}\end{array}$

\begin{tabular}{lllll}
\hline 0.0 & 1.63 & 2 & $18.0^{1}$ & 323
\end{tabular}

$\begin{array}{lllll}1.63 & 9.03 & 2 & 13.1^{1} & 34\end{array}$

$\begin{array}{llllll}0.0 & 9.03 & 4 & 42.8 & 1.83 \times 10^{3}\end{array}$

$\begin{array}{lllll}0.0 & 1.63 & 2 & 18.0^{1} & 323\end{array}$

$\begin{array}{llllll}1.63 & 9.99 & 2 & 15.7^{2} & 49\end{array}$

\begin{tabular}{lllll}
0.0 & 9.99 & 4 & 128 & $1.64 \times 10^{4}$ \\
\hline 0.0 & 7.16 & 3 & $45.7^{1}$ & $2.08 \times 10^{3}$
\end{tabular}


TABLE V-4.

Trangition strengths used in CCLA calculations.

$\begin{array}{lllll}E_{1} & E_{q} & 1 & M\left(e \mathrm{fm}^{\prime}\right) & \mathrm{B}(\mathrm{E} l ; i-1)\left(\mathrm{e}^{2} \mathrm{fm}^{2}\right) \\ 7.16 & 10.26 & 2 & 10.7^{1} & 16 \\ 0.0 & 10.26 & 5 & >805 \text { or } 905^{5} & 6.48 \times 10^{6} \text { or } 8.19 \times 10^{6}\end{array}$

1.) These matrix elements were fixed by other data. For transitions starting with the ground state the results of DWIA calculations given in Table V-3 were used. All other transition strengths were taken from the latest compilation (AJ-87).

2.) This matrix element was assumed to be equal to the $0.0 \rightarrow 7.16$ matrix element.

3.) $\mathrm{M}$ is defined in the text for E0 and $\mathrm{E} 1$ transitions.

4.) This matrix element was assumed to be equal to the 0.0-4.25 matrix element.

5.)Depending on whether the interference is constructive or destructive. 


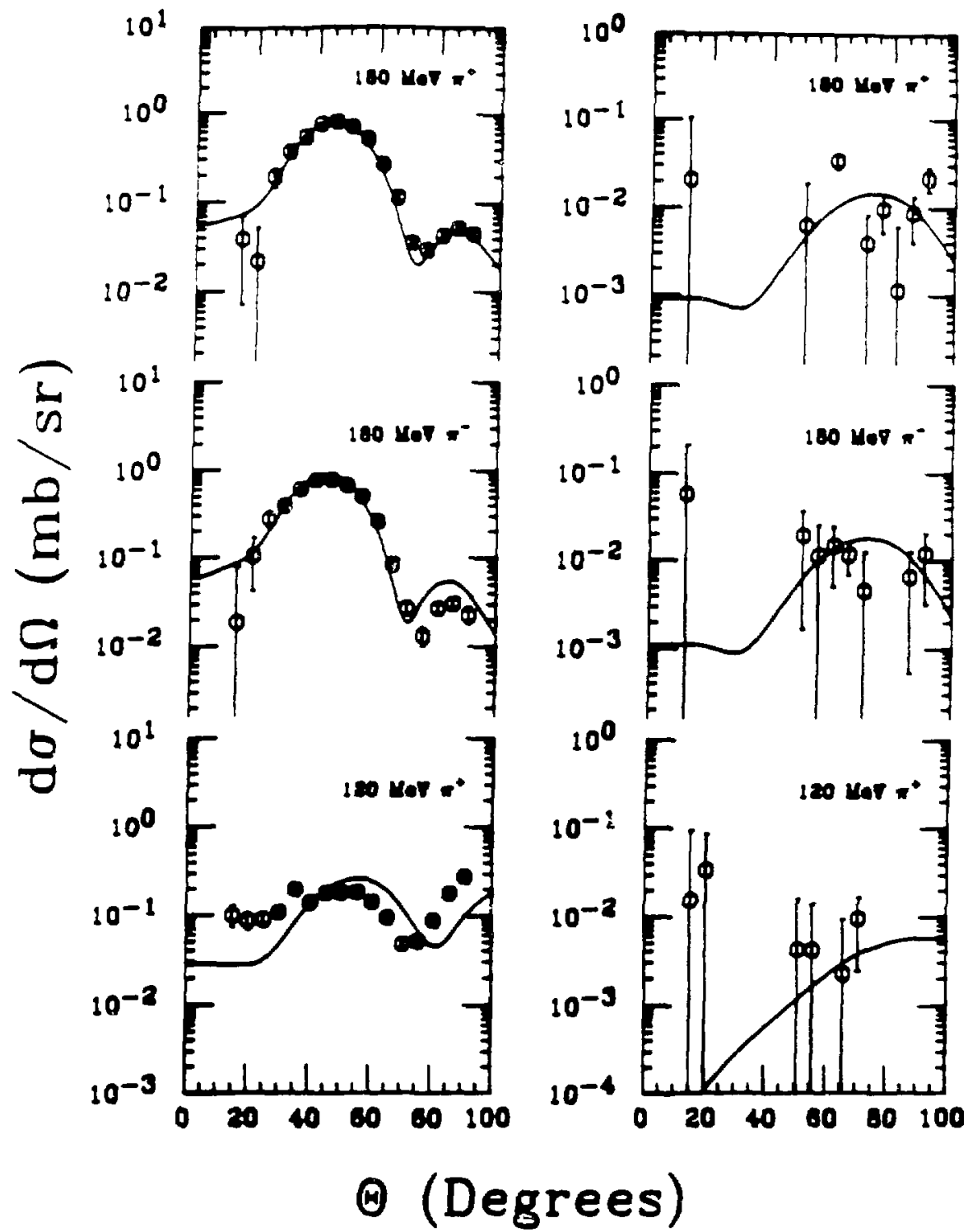

Figure V-11.

CCLA calculations to the $4.25-\mathrm{MeV} 4^{+}$(left column) and the $8.78-\mathrm{MeV}^{+}$ (right column). The top graph in each column is $180 \mathrm{MeV} \pi^{+}$, the middle $180-\mathrm{MeV} \pi^{-}$, and the bottom $120-\mathrm{MeV}^{+}$. 


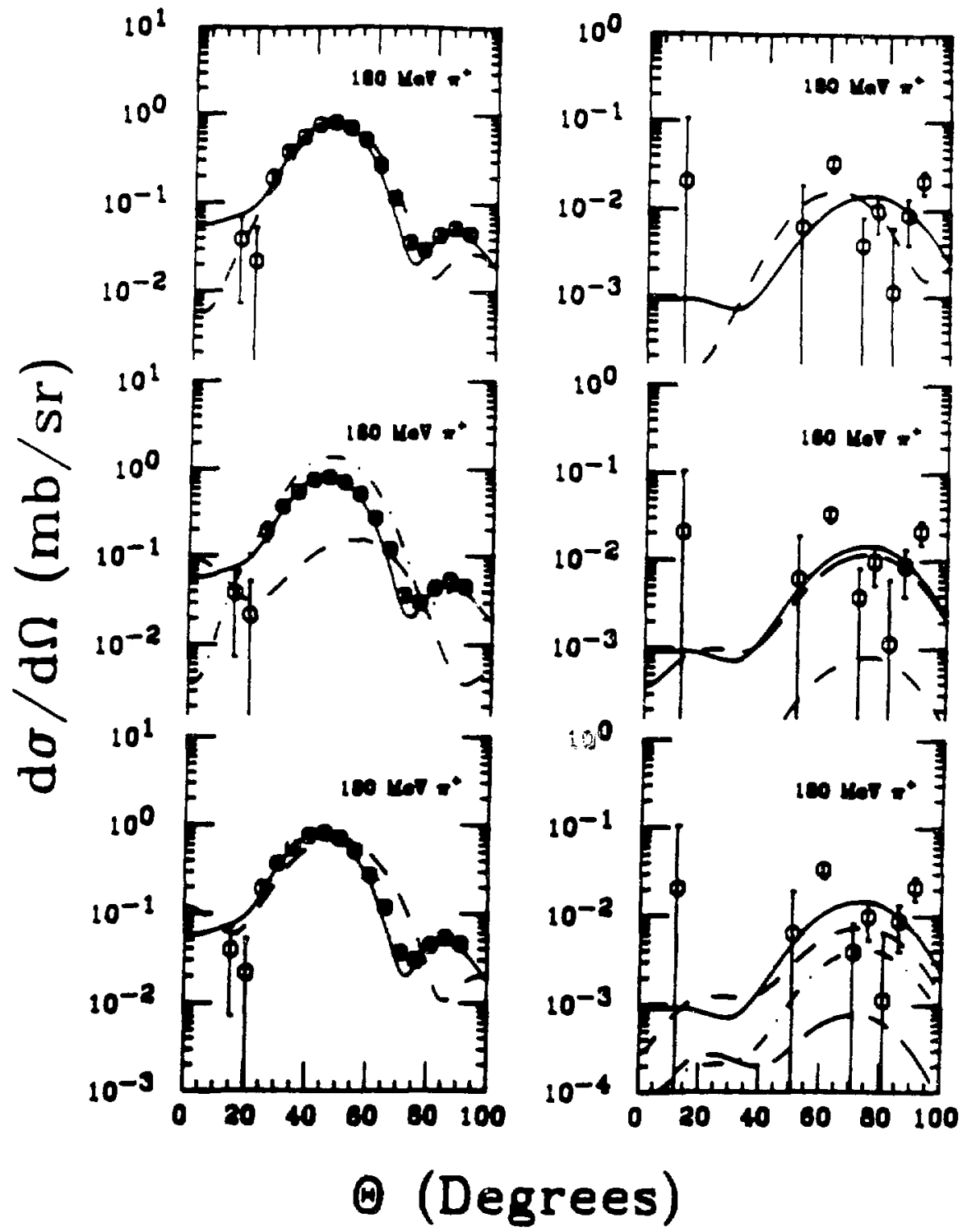

Figure V.12.

CCLA calculations to the $4.25-\mathrm{MeV}^{+}$(left column) and the $8.78-\mathrm{MeV}^{+}$ (right column). All of the craphs are $180 \mathrm{MeV} \pi^{+}$. The solid curve is the complete CCLA calculation in all 6 graphs. The dashed curves in the top graphs are DWPI calculation. In the middle graphs the danhed curves show the contributions of the difierent routes. In the bottom graphs the dashed curves shor the effect of changing the relative phase. 
very weak. For the $4^{+}$calculation the choice of phase, +1 or -1 , between the routes was straight-forward as is shown in the bottom graph. The dashed line shows the calculation with the opposite phase and the strength of the E4 varied to reproduce the overall magnitude of the data. Both the maximum and the minimum are at too large an angle. For the $6^{+}$the phases chosen were those that gave the largest overall magnitude. With the addition of a direct $0^{+}-6^{+}$route any choice of phase can be made to fit the data if sufficient E6 strength is included.

$$
\text { V-3. } K^{\pi}=\mathrm{O}_{2}^{+} \text {band }
$$

Three peaks seen in this experiment correspond to states in the $\mathrm{K}^{\pi}=\mathrm{O}_{2}^{+}$ band. They are the $6.73-\mathrm{MeV} \mathrm{O}^{+}$, the only $0^{+}$excited state seen, the $7.42-\mathrm{MeV}^{+}$, which was observed in electron scattering to have an anomalous form factor (MI72), and the 9.99-MeV 4+. Angular distributions and DWIA calculations for the $2^{+}$and $4^{+}$are shown in Figure $\mathrm{V}-13$. The $7.42-\mathrm{MeV}$ peak has an anomalous angular distribution, as in electron scattering.

The $l=4$ calculation does a reasonable job of reproducing the $9.99-\mathrm{MeV}$ data, but the first maximum and minimum appear to be at a smaller angle than the DWIA calculation predicts. An $l=3$ gives almost as good a fit to the data. The 9.99-MeV $4^{+}$has a strong E2 branch to the $1.63-\mathrm{MeV} \mathrm{2}^{+}$, so two-step calculations using the 1.63-MeV state as the intermediate state have been done. The couplings used are shown in Figure V-14 and their strengths given in Table V-4. The strength of the $l=4$ transition was chosen to fit the data. These calculations significantly improve the fit to the $9.99-\mathrm{MeV}$ state as shown in the left column of Figure V-15. The CCIA calculation does a very good job of fitting the $9.99-\mathrm{MeV}$ data. The top graph of the right column of Figure V-15 shows a comparison of the CCIA (solid line) and DWIA (dashed line). The middle graph shows the 


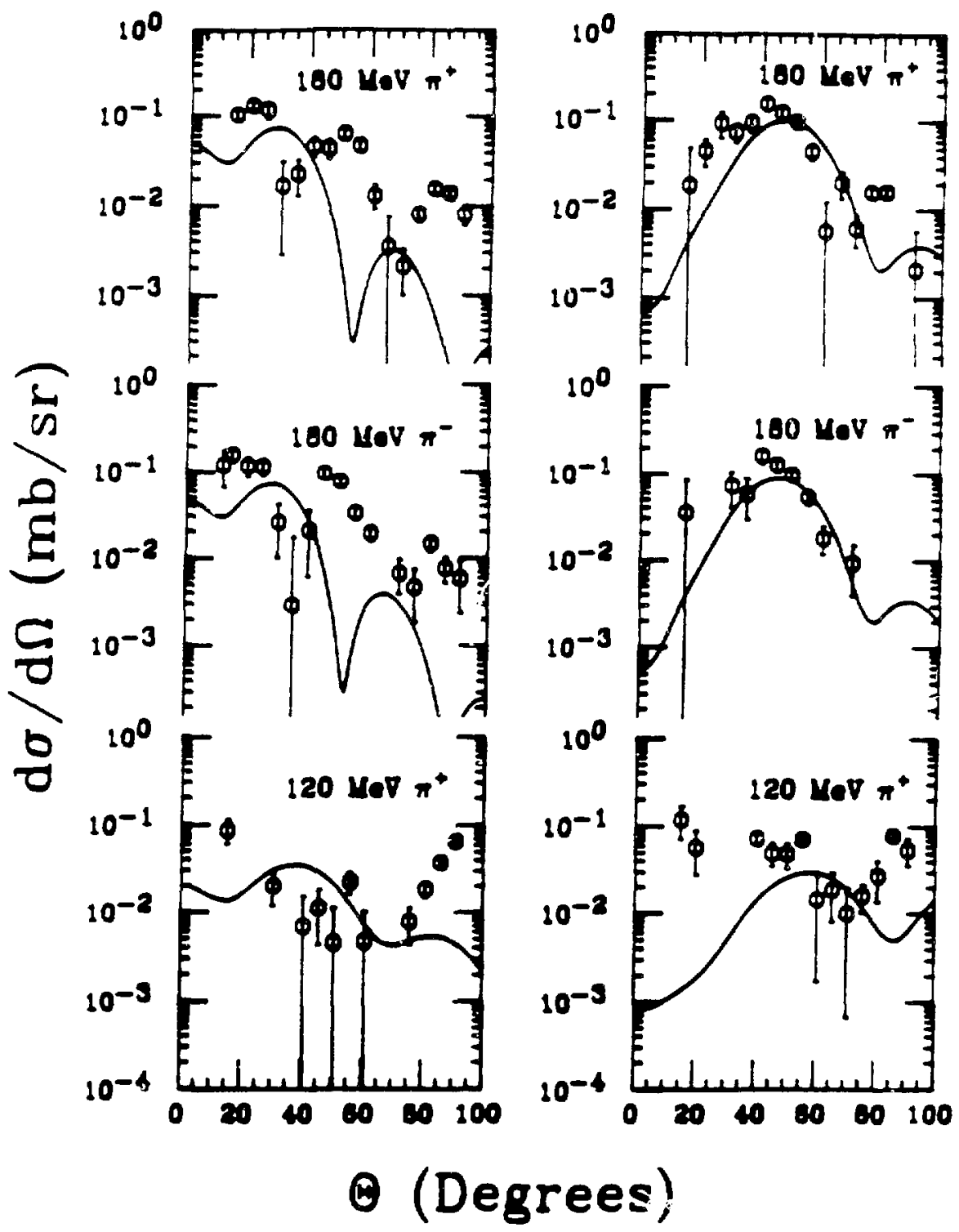

Figure V-13.

Angular distributions for the $7.42-\mathrm{MeV}^{+}$(left column) and the $9.29-\mathrm{MeV}_{4}^{+}$ (right column). The top sraph in each column is $180-\mathrm{MeV} \pi^{+}$, the middle $180-\mathrm{MeV} \pi^{-}$, and the bottom $120-\mathrm{MeV} \pi^{+}$. The curres are DWPI calculations. 


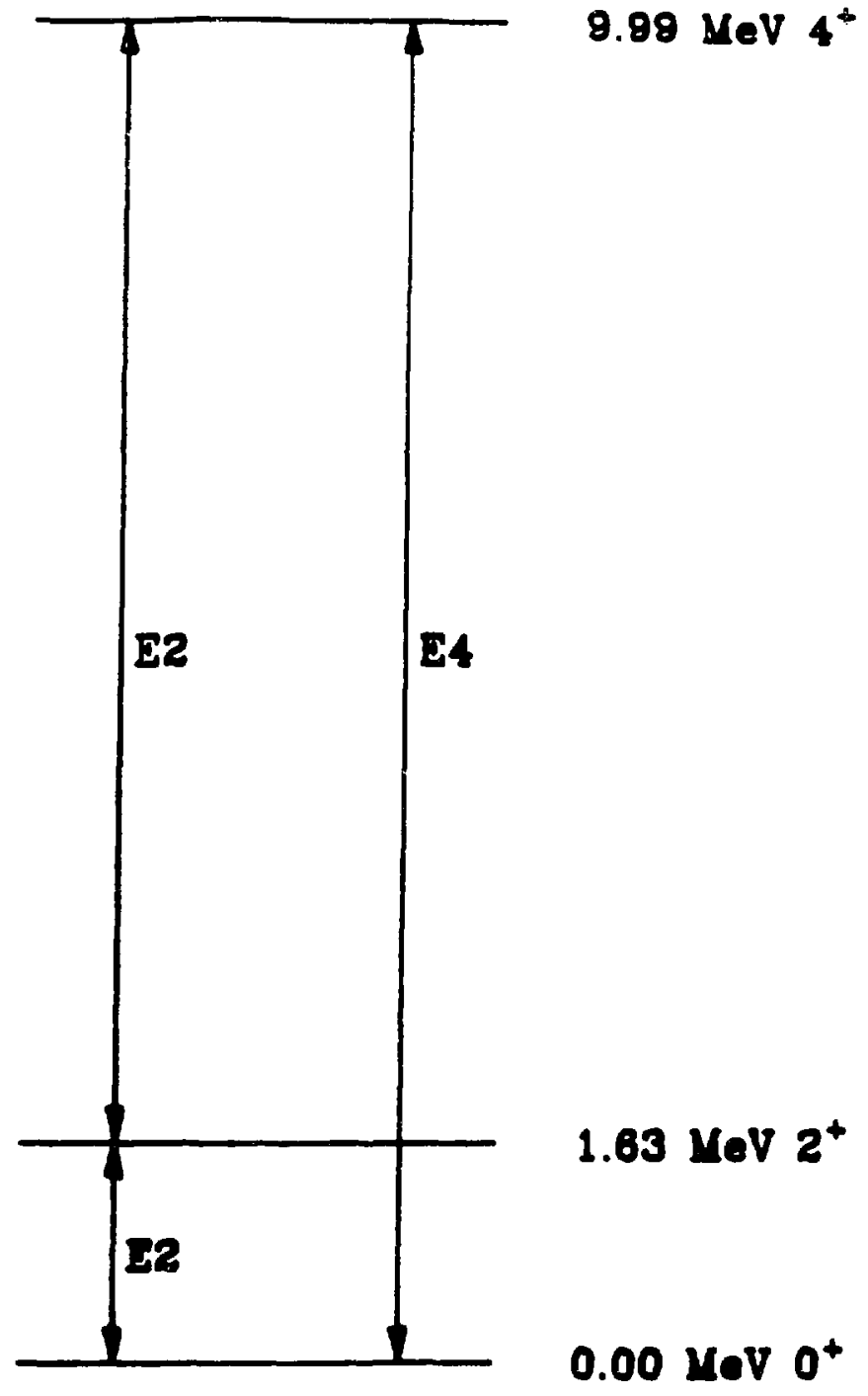

Figure V-14.

Couplings used in the CCLA calculations for the $9.99 \mathrm{MeV} 4^{+}$state. 


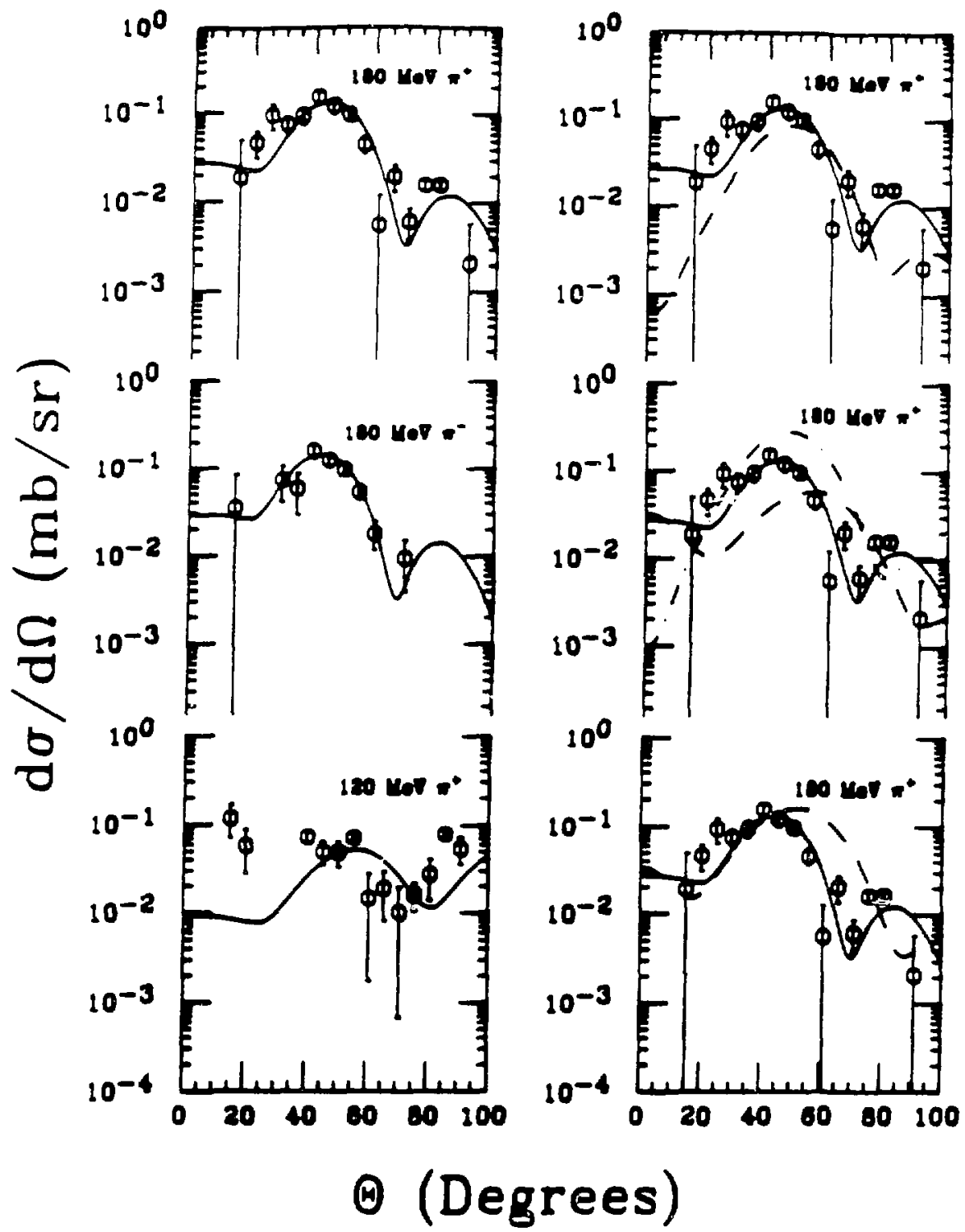

Figure V-15.

CCIA calculations to the $9.92-\mathrm{MeV}^{4}$. The solid curve is the complete CCLA calculation in all 6 graphs. The top graph in left column is $180-\mathrm{MeV} \pi^{+}$, the middle $180-\mathrm{MeV}^{-}$, and the bottom $120-\mathrm{MeV}^{+}$. In the right column all of the graphs are $180-\mathrm{MeV} \pi^{+}$. The dashed curve in the right top sraph is a DWPI calculation. In the right middle graph the dashed curves show the contributions of the different routes. In the right bottom graph the dashed curve shows the effect of changing the relative phase. 
contributions of the direct (dot-dashed line) and $2 \mathrm{P} 1$ (dashed line) routes and their sum (solid line). Again destructive interference was necessary to fit the angular distribution. The bottom graph shows the results with constructive interference (dashed line). Constructive interference pushes the maximum and minimum to larger angles.

Coupled channels contributions are also important for the 6.73- and 7.42-MeV peaks. The important couplings are shown in Figure V-16. For the 7.42-MeV 2+ the two-step route, the solid curve in Figure V-17, is clearly non-negligble, especially around the minima. Unfortunately, it is not possible to fit the data including a direct route using the standard collective-model transition density. The dashed curves in Figure V-17 show the two-step and direct routes added -nd subtracted coherently, limiting the strength of the direct route to the upper limit in the compilation (AJ-87). If the strength of the direct route is increased, the CCIA calculation can be made to reproduce the magnitude of the data, the dot-dashed curves in Figure V-17, but not the shape of the angular distribution. The $7.42-\mathrm{MeV}$ state also has a weak decay to the $4.25-\mathrm{MeV}^{+}$, but that route is at least an order of magnitude smaller than the lowest $180-\mathrm{MeV} \pi^{+}$data point. No decay from the 7.42-MeV state to the $6.72-\mathrm{MeV} 0^{+}$has been seen, (AJ-87), but since both states are in the game rotational band a two-step calculation using the $6.73-\mathrm{MeV} \mathrm{n}^{+}$as the intermediate state was also done. The transition between the 7.42-MeV state with the 6.73-state has to be at least 3 times the strength of the transition between the $1.63-\mathrm{MeV}$ state and the ground state to have any significant effect. Microscopic calculations, discussed in section V-10, predict an anomalously shaped angular distribution with a shape similar to the data, Figures V-49 and V-50. NEWCHOP (MO-01) is unable to calculate a microscopic transition density. 


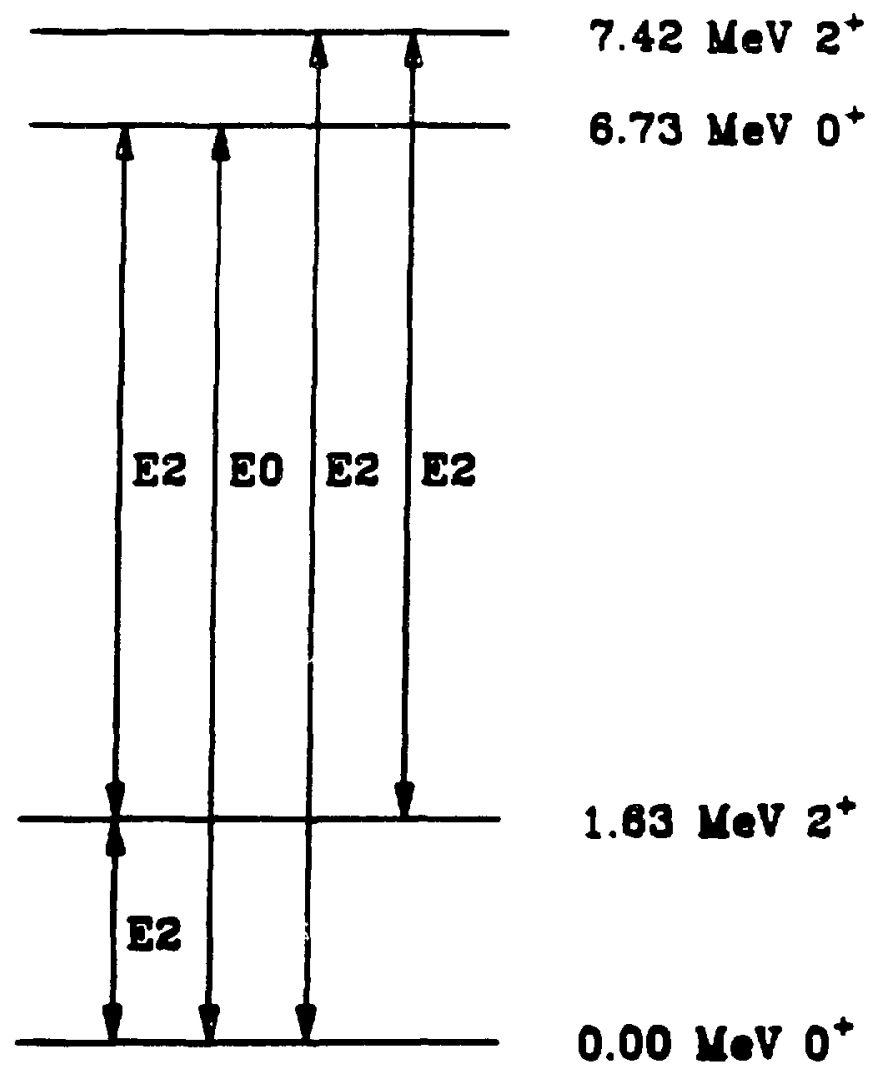

Figure V-16.

Couplings used in the CCIA calculation for the $6.73-\mathrm{MeV} \mathrm{O}^{+}$and $7.42-\mathrm{MeV}$ $2^{+}$states. There is no couplins between the 6.73- and the 7.42-MeV states. 


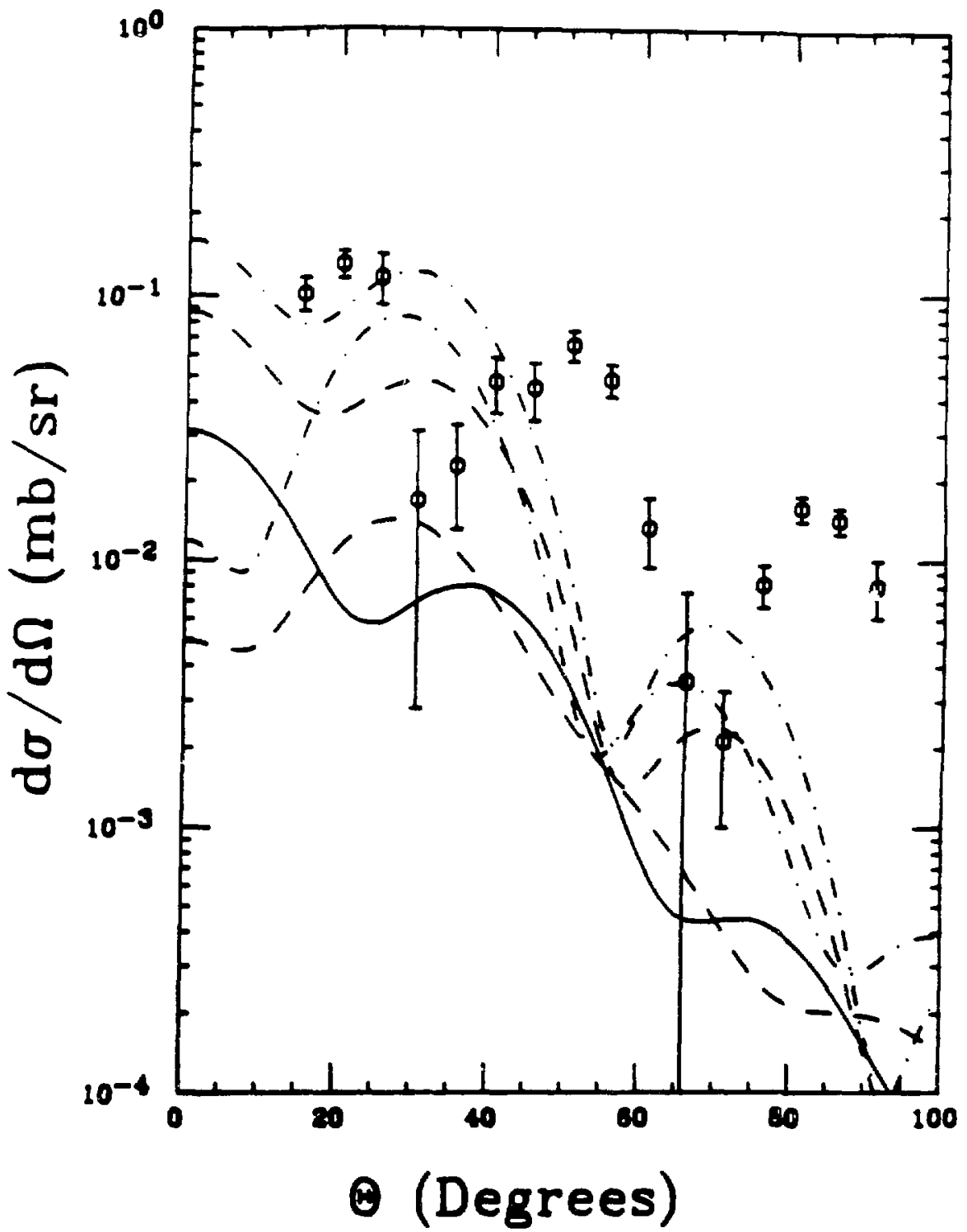

Figure V-17.

CCLA calculations to the $7.42-\mathrm{MeV}^{+}$for $180 \mathrm{MeV} \pi^{+}$incident beam. The solid curve is the $2 \mathrm{P1}$ route. The dashed curves are the $2 \mathrm{P1}=\mathrm{DIR}$ routes, with the direct strength at the upper limit in the compilation, (AJ-87). The dot-dashed curves are the $2 \mathrm{P} 1 \pm \mathrm{DIR}$ routes, with the direct strength increased to fit the macnitude of the date. 


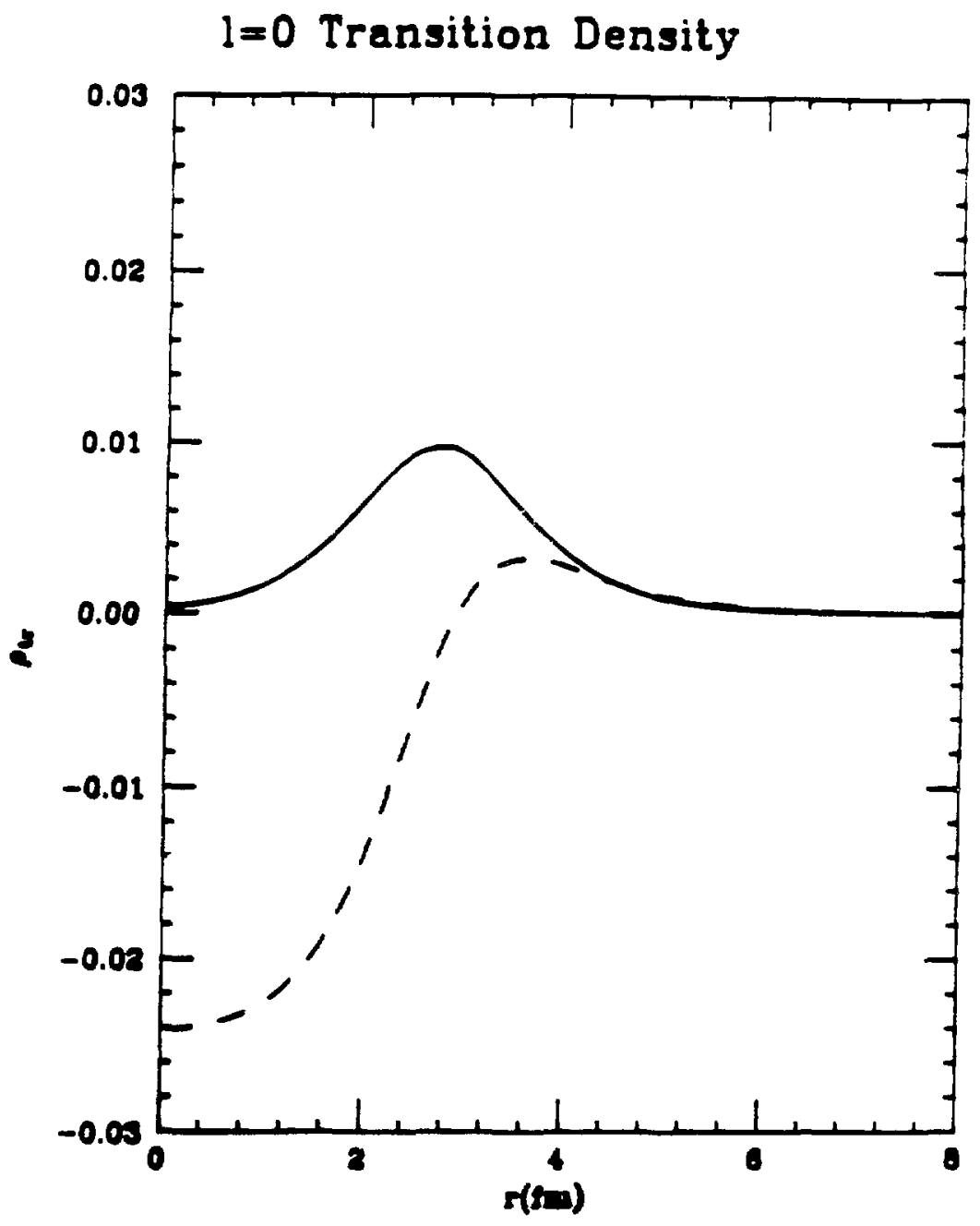

Figure V-18.

$l=0$ transition densitie used for the 6.73- $\mathrm{MeV} \mathrm{O}^{+}$. 


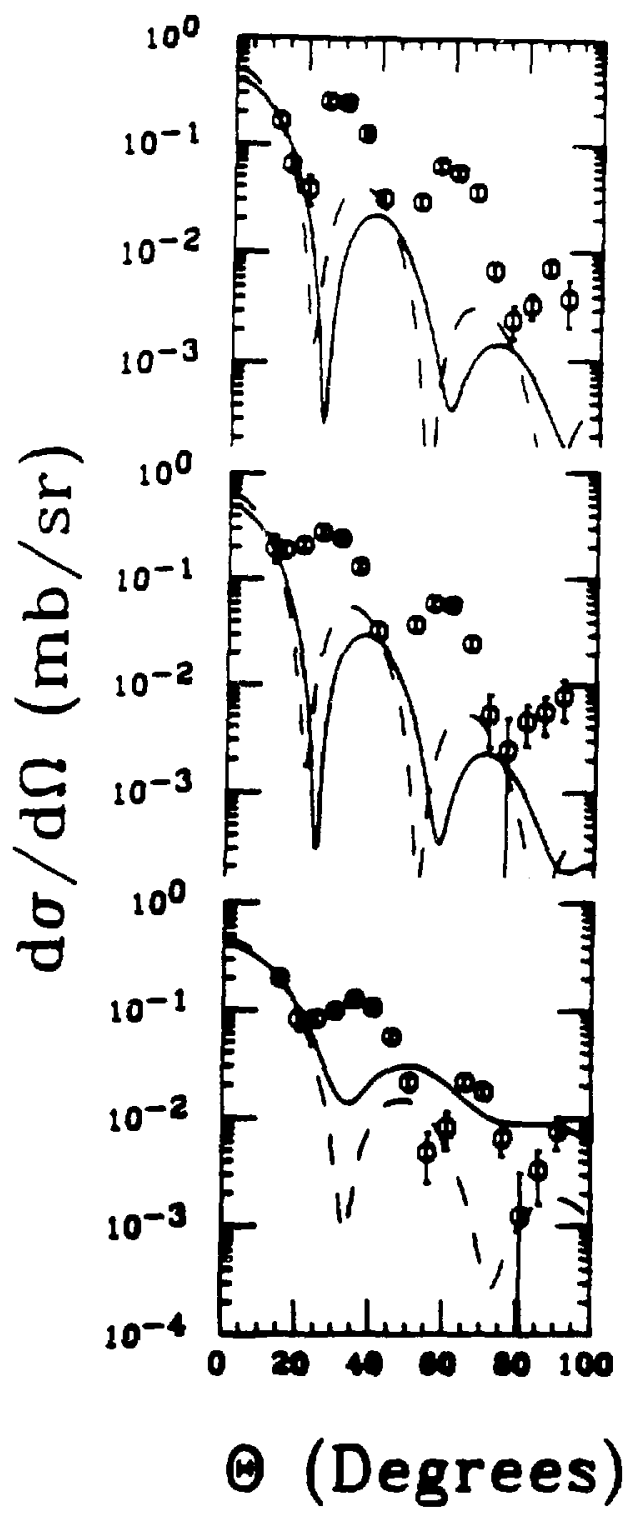

Figure V-19.

Angular distributions for the 6.73- $\mathrm{MeV} 0^{+}$. The top craph is $180 \mathrm{MeV} \pi^{+}$, the middle $180-\mathrm{MeV} \pi^{-}$, and the bottom $120-\mathrm{MeV} \pi^{+}$. The solid curre uses the standard collective-model transition density. The danbed curve usea the transition density of AU-71. 
The standard collective transition density is incorrect for $1=0$ transitions, as was discussed in Chapter 4. Figure V-18 shows the standard collective transition density (solid line) and the transition density of Auerbach (AU-71) (dashed line). While these transition densities look very different they produce very similar results for the angular distributions as shown in Figure V-19. This is probably because the pion sees only the surface of the nucleus and on the surface these transition densities are very similar. In electron scattering a phenomenological transition density of the form

$$
\rho_{t r}(r)=\rho_{0}\left(1+a r^{2}+b r^{4}+c r^{0}\right) \exp \left(-\frac{r^{2}}{\beta^{2}}\right)
$$

was used (MI-72). With the constraint that $c=0$, then knowing $M$ and $R_{t r}$, where

$$
M=\left\langle r^{2}\right\rangle_{t r}=(4 \pi)^{4} \int_{r=0}^{\infty} r^{2} \rho_{t r} r^{2} d r \quad(M I-72)
$$

and

$$
\mathrm{R}_{\mathrm{tr}}^{2}=\frac{\left\langle\mathrm{r}^{4}\right\rangle_{\mathrm{tr}}}{\left\langle\mathrm{r}^{2}\right\rangle_{\mathrm{tr}}}(\mathrm{MI}-72)
$$

fixes $\rho_{0}$, $a$, and $b$. Using $M=7.37 \pm 1.97 \mathrm{fm}^{2}$ and $R_{t r}=5.73 \pm 1.30 \mathrm{fm}(M I-72)$ or $\mathrm{M}=5.85 \pm 1.5 \mathrm{fm}^{2}$ (SI-73) from electron scattering, a DWIA calculation can reproduce the magnitude of the data. The $0^{+} \rightarrow 2^{+}-0^{+}$(2P1) two-step route is important near the minima of the data. Varying these parameters, allowing terms up to $r^{\circ}$, and including the two-step route gives the curves shown Figure V-20. The best fit finds $M=5.27 \pm 0.04 \mathrm{fm}^{2}$ and $R_{t r}=5.95 \pm 0.07 \mathrm{fm}$, shown in the left column of Figure V-20. The errors are statistical errors only. The top graph in the right column shows the contribution of the direct and the bottom shows the two-step for the $180-\mathrm{MeV} \pi^{+}$calculation. If $\mathrm{c}$ is set equal to zero, the fit is not as good, although 


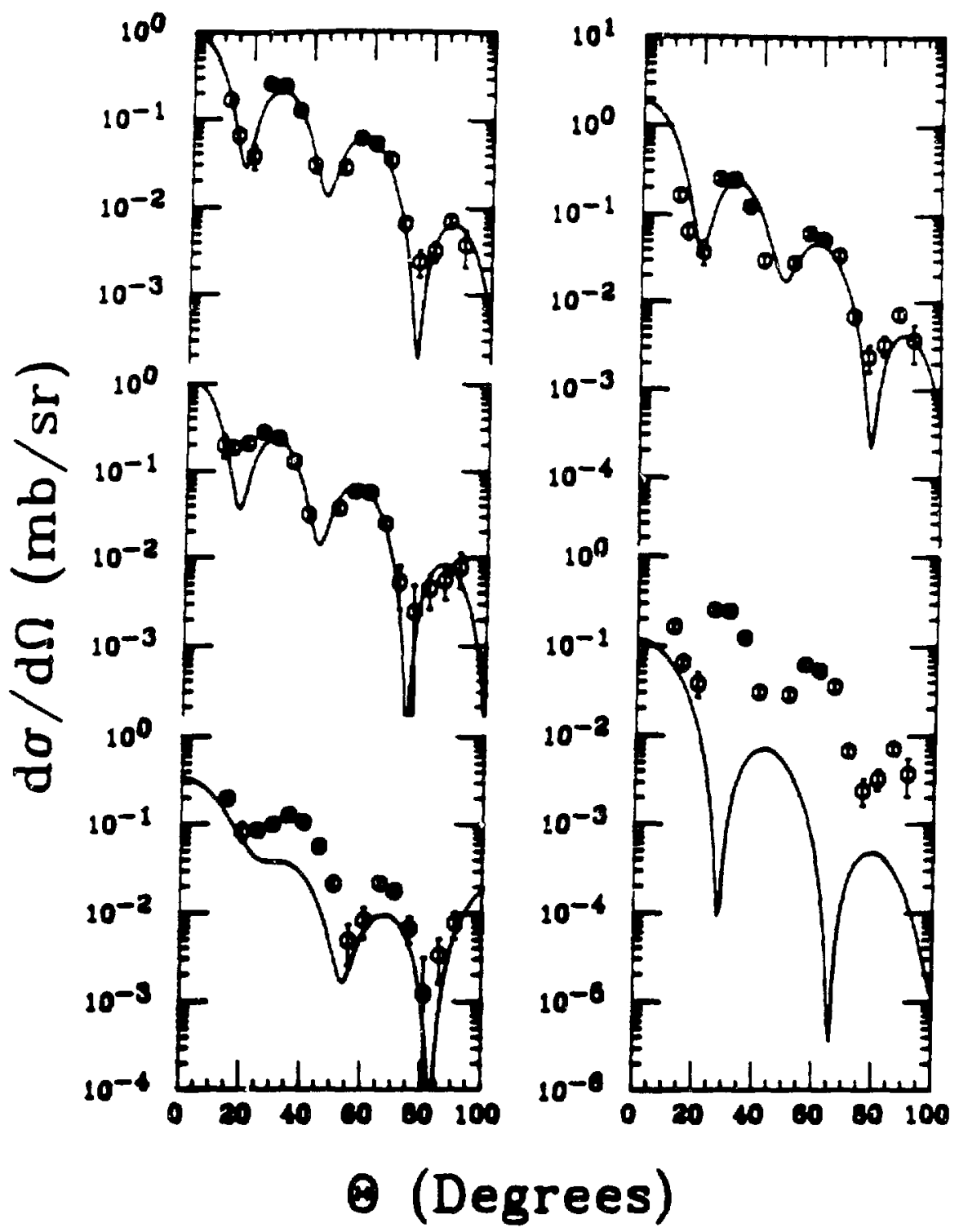

Figure V-20.

CCLA calculation to the $6.73-\mathrm{MeV} \mathrm{O}^{+}$. The top graph in left column is 180 $\mathrm{MeV} \pi^{+}$, the middle $180-\mathrm{MeV} \pi^{-}$, and the bottom $120 \mathrm{MeV} \pi^{+}$. In the right column all of the graphs are $180-\mathrm{MeV}^{+}$. The curve in the right top graph is the direct component of the CCLA calculation. In the right bottom graph the curve is the 2P1 component of the CCIA calculation. 
still very good, and the extracted $M$ and $R_{t r}^{2}$ are changed by an amount much less than statistical error of the original fit.

\section{$\mathrm{V}-4 \cdot \mathrm{K}^{\pi}=\mathrm{O}_{3}{ }^{+}$band}

The 7.83- $\mathrm{MeV}^{+}$and 9.03- $\mathrm{MeV} 4^{+}$members of the $\mathrm{K}^{\pi}=0_{3}^{+}$band are shown in Figure V-21. DWIA calculations reproduce both angular distributions reasonably well, and the extracted matrix elements are given in Table V-3. The first maximum and minimum for both states are at larger angles than the calculation gives. Both states have a strong $\mathrm{E} 2$ decay to the $1.63-\mathrm{MeV} 2^{+}$, so two-step calculations were done. The $0^{+} \rightarrow 2^{+} \rightarrow 2^{+}$calculation is negligibly small and unable to improve the fit. On the other hand, $0^{+}-2^{+}-4^{+}$is important, as shown in Figure V-22. The dashed line in the middle graph of the second column shows that the contribution of this route is comparable to the contribution of the direct route, the dot-dashed line. The left column shows the results of the coupled-channels calculation using both the $2 \mathrm{P} 1$ and direct routes, varying the strength of the direct route to fit the data. The strengths used are given in Table V-4. Constructive interference was necessary to push the maximum and minimum to larger angle and fit the data.

\section{V-5. $K^{\pi}=0_{4}^{+}$band}

It is somewhat surprising that the $2^{+}$and $4^{+}$states in this band were excited in pion inelastic scattering. This band is believed to result from the excitation of 2 or 4 particles into the fp shell. Since the pion's interaction with the nucleus is a one-body operator, this state should not be excited unless its wave function has some (sd) components or that of the ground state has some $(f p)^{2}$ or $(f p)^{4}$ components. Both of these states have large $\alpha$ widths and no $\gamma$ decays are known for 


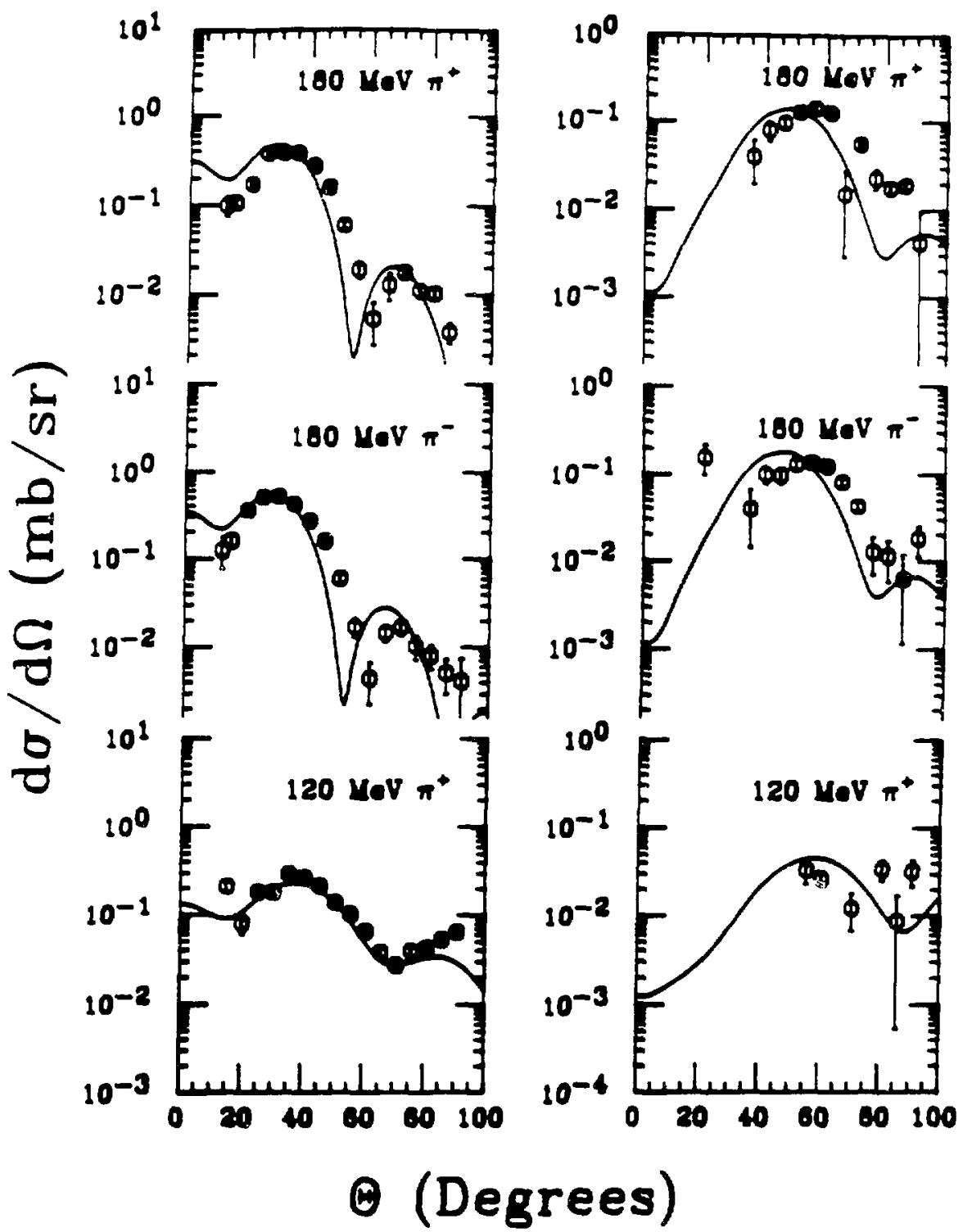

Figure V-21.

Angular distributions for the $7.83-\mathrm{MeV}^{+}$(left column) and the $9.03-\mathrm{MeV}_{4}^{+}$ (right column). The top graph in each column in $180 \mathrm{MeV} \pi^{+}$, the middle $180-\mathrm{MeV} \pi^{-}$, and the bottom $120-\mathrm{MeV} \pi^{+}$. The curves are DWPI calculations. 


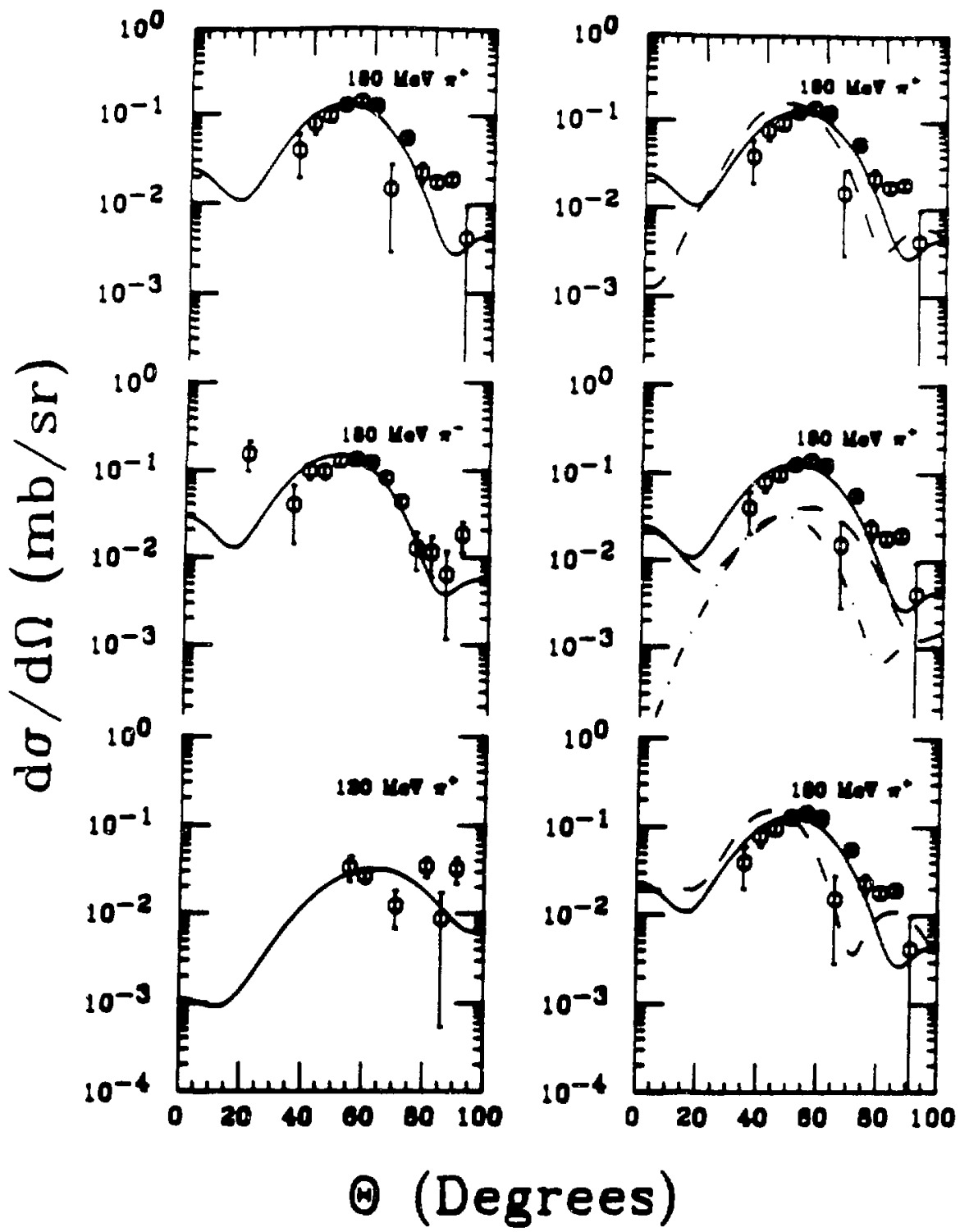

Figure V-22.

CCLA calculations to the $9.03-\mathrm{MeV} 4^{+}$. The top graph in left column is 180 $\mathrm{MeV} \pi^{+}$, the middle $180-\mathrm{MeV} \pi^{-}$, and the bottom $120-\mathrm{MeV} \pi^{+}$. In the right column all of the graphs are $180-\mathrm{MeV}^{+}$. The dashed curve in the right top graph is a DWPI calculation. In the right middle graph the dashed curves show the contributions of the different routes. In the right bottom craph the dashed curve shows the effect of changing the relative phase. 
either of them. The compilation, $(\mathrm{AJ}-87)$, lists $\mathrm{E}_{\mathrm{x}} \approx 8.8 \mathrm{MeV}$ and $\Gamma>800 \mathrm{keV}$ for the $2^{+}$and $E_{x}=10.80 \pm 0.075 \mathrm{MeV}$ and $\Gamma=350 \mathrm{keV}$ for the $4^{+}$. Values of $E_{x}=9.00 \pm 0.18 \mathrm{MeV}$ and $\Gamma=800 \mathrm{keV}$ for the $2^{+}$and $E_{x}=10.79 \mathrm{MeV}$ and $\Gamma=350$ $\mathrm{keV}$ for the $4^{+}$fit the current data best. Because of their large widths these states have not been extensively studied. Both the $2^{+}$and $4^{+}$, along with the $0^{+}$at 8.3 $\mathrm{MeV}$ which is not seen here, are seen in ${ }^{10} \mathrm{O}(\alpha, \alpha)$ and ${ }^{19} \mathrm{~F}\left({ }^{3} \mathrm{He}, \mathrm{d}\right)$, and the $4^{+}$has also been seen in ${ }^{10} \mathrm{O}\left({ }^{\circ} \mathrm{Li}, \mathrm{d}\right),(\mathrm{AJ}-87)$. Mixing between the $8.3-\mathrm{MeV} \mathrm{O}^{+}$and the 6.73- $\mathrm{MeV}^{+}$and between the $9.0-\mathrm{MeV} 2^{+}$and the $7.42-\mathrm{MeV}^{+}$can explain the $\left({ }^{3} \mathrm{He}, \mathrm{d}\right)$ data (FO-72a, FO-76), but that mixing is unable to explain the present data. The structure of the $9.00-\mathrm{MeV}$ state is discussed in more detail in Appendix 2.

Angular distributions for these states are shown in Figure V-23 with DWIA calculations. The matrix elemenis extracted from these fits are given in Table V-3. The DWIA calculation gives a maximum and a minimum at a larger angle than the data for the $2^{+}$. It has been shown in previous sections that CCIA calculations can shift the maximum and minimum, but since no couplings between this state and other state are known it is not possible to do a useful calculation. Figure V-24 compares the $1.63-\mathrm{MeV} 2^{+}$with the $9.0-\mathrm{MeV}^{+}$. The solid curve is the standard DWLA calculation; the dashed curve is a calculation using a transition density which has its peak at a radius that is $\approx 25 \%$ larger than the standard transition density. Increasing the radius pushes the maximum and minimum to smaller angle and improves the fit. If this band is formed by exciting 2 or 4 particles to the fp shell, then the transition density should peak at a larger radius, so the data presented here appears to be consistent with the belief that these states are either $(f p)^{4}$ or $(f p)^{2}(s d)^{2}$ configurations. 


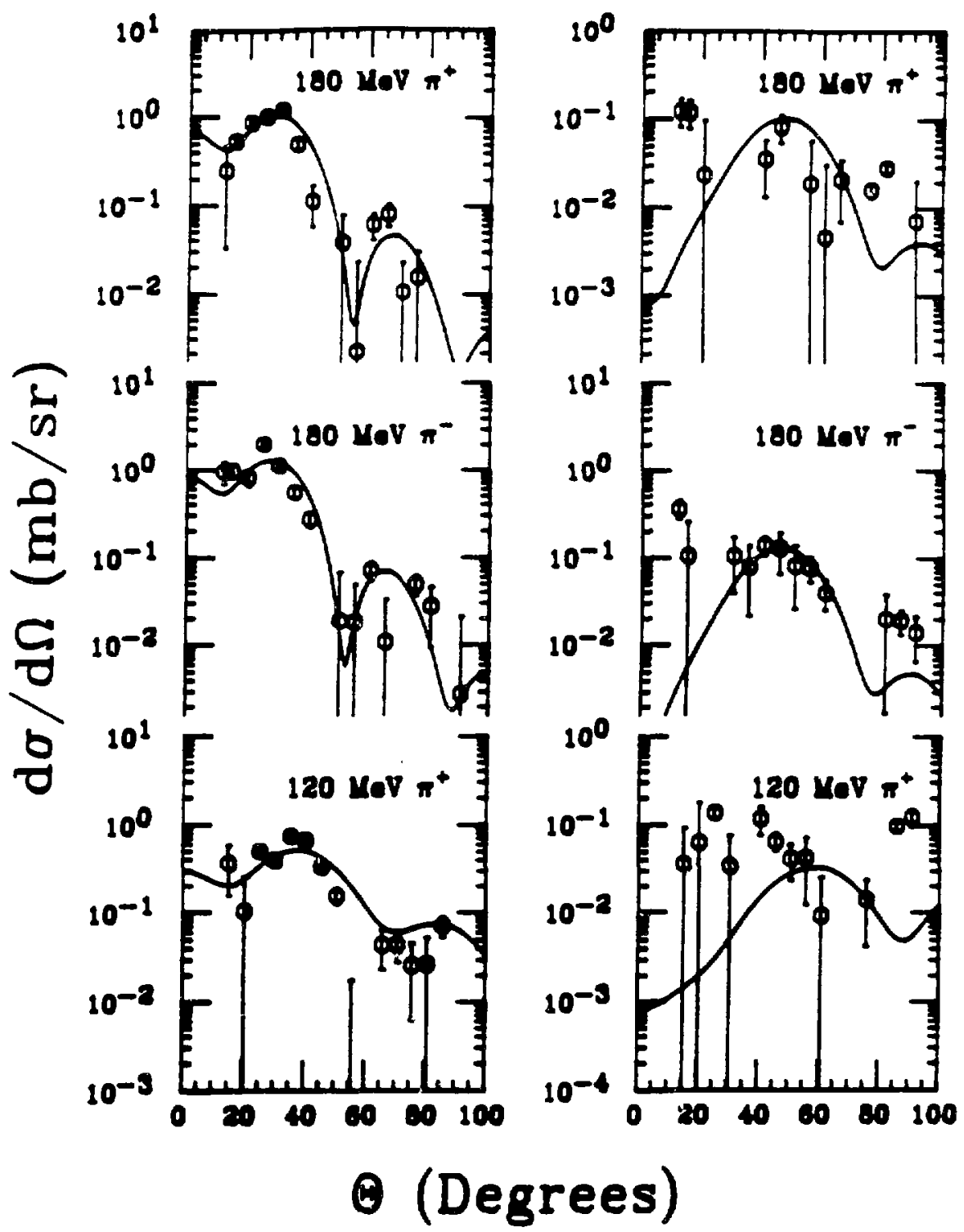

Figure V-23.

Angular distributions for the $9.00-\mathrm{MeV}^{+}$(left column) and the $10.79 \mathrm{MeV}$ $4^{+}$(right column). The top graph in each column is $180-\mathrm{MeV} \pi^{+}$, the middle $180-\mathrm{MeV} \pi^{-}$, and the bottom $120-\mathrm{MeV} \pi^{+}$. The curres are DWPI calculations. 


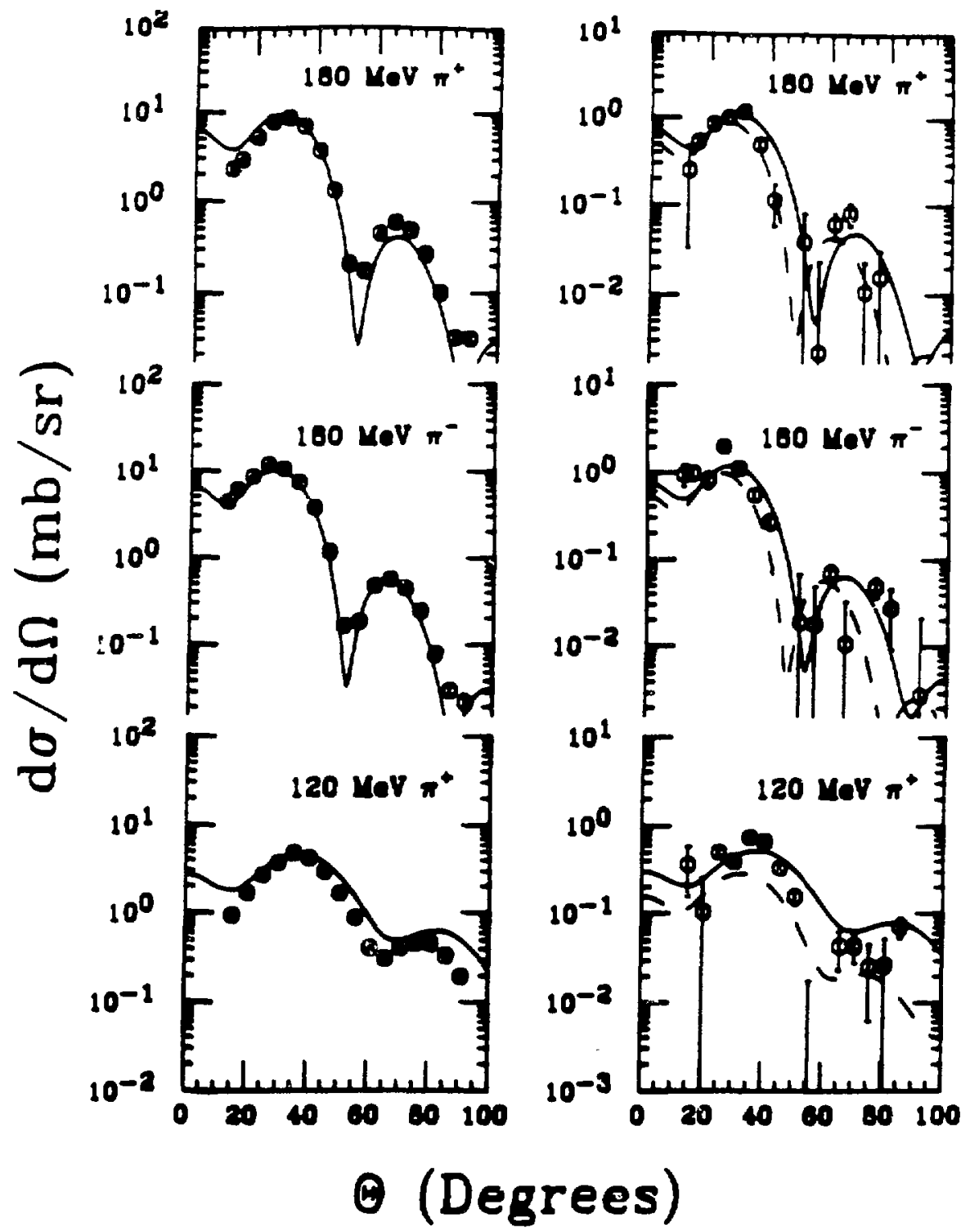

Firure V-24.

Angular distributions for the $1.63-\mathrm{MeV}^{+}$(left column) and the $9.00-\mathrm{MeV}^{+}$ (right column). The top graph in each column is $180-\mathrm{MeV} \pi^{+}$, the middle $180-\mathrm{MeV} \pi^{-}$, and the bottom $120 \mathrm{MeV} \pi^{+}$. The curres are DWPI calculations. 


\section{V-6. $K^{\pi}=2^{-}$band}

Several states from this band are present in our data. The $3^{-}$at $5.62 \mathrm{MeV}$ is one of the strongest states in the spectrum. There is a peak at $8.45 \mathrm{MeV}$ which may correspond to the $5^{-}$. The $4.97-\mathrm{MeV}^{-}$is seen, although the $7.00-\mathrm{MeV}^{-}$is not. Unnatural-parity states are generally weak in pion inelastic scattering on even-even nuclei because the spin-flip part of the $\pi$-nucleus interaction is very weak. It is so small it is dropped in DWPI (EI-76a), so it is not possible to do a DWIA calculation to this peak using a collective transition density. The spin-flip term is included in PIPIT (El-76b) so a microscopic calculation can be done to it. The microscopic calculations are discussed in Section V-10. This state is coupled to the $3^{-}$at $5.62 \mathrm{MeV}$ with a strong E2, so it may be excited through a two-step route using the $3^{-}$as the intermediate state. NEWCHOP (MO-01) assumes that all states in the calculation have the same $K$, which for natural-parity states is not a problem, but a $2^{-}$state cannot have $\mathrm{K}=0$ so NEWCHOP calculates zero for the cross section.

The 5.62- and 8.45-MeV angular distributions are shown in Figure V-25. The curves are DWIA calculations using DWPI; the extracted M's are in Table V-3. The $5.62-\mathrm{MeV}$ peak is well fitted by the $1=3$ calculation. The $8.45-\mathrm{MeV}$ peak is clearly not a simple $l=5$ transition. The $8.45-\mathrm{MeV}$ angular distribution resembles an $l=2$ more than an $l=5$. The left column of Figure $V-26$ shows a fit to the $8.45-$ $\mathrm{MeV}$ peak with an $l=2$ calculation, which fits reasonably well. The $\mathrm{M}$ extracted from this fit is included in Table V-3. The $8.45-\mathrm{MeV}^{-}$state is known to have strong E2 $\gamma$ decay to the $5.62-\mathrm{MeV}^{-}$, (AJ-87), given in Table V-4. A two-step calculation using the route $\mathrm{O}^{+} \rightarrow 3^{-} \rightarrow 5^{-}$, labelled $3 \mathrm{M} 1$, overpredicts the large angle data by a factor of two as shown in Figure V-27. The dashed curve in the middle graph in the right column is the $3 \mathrm{M} 1$ calculation. If a strong direct component is 


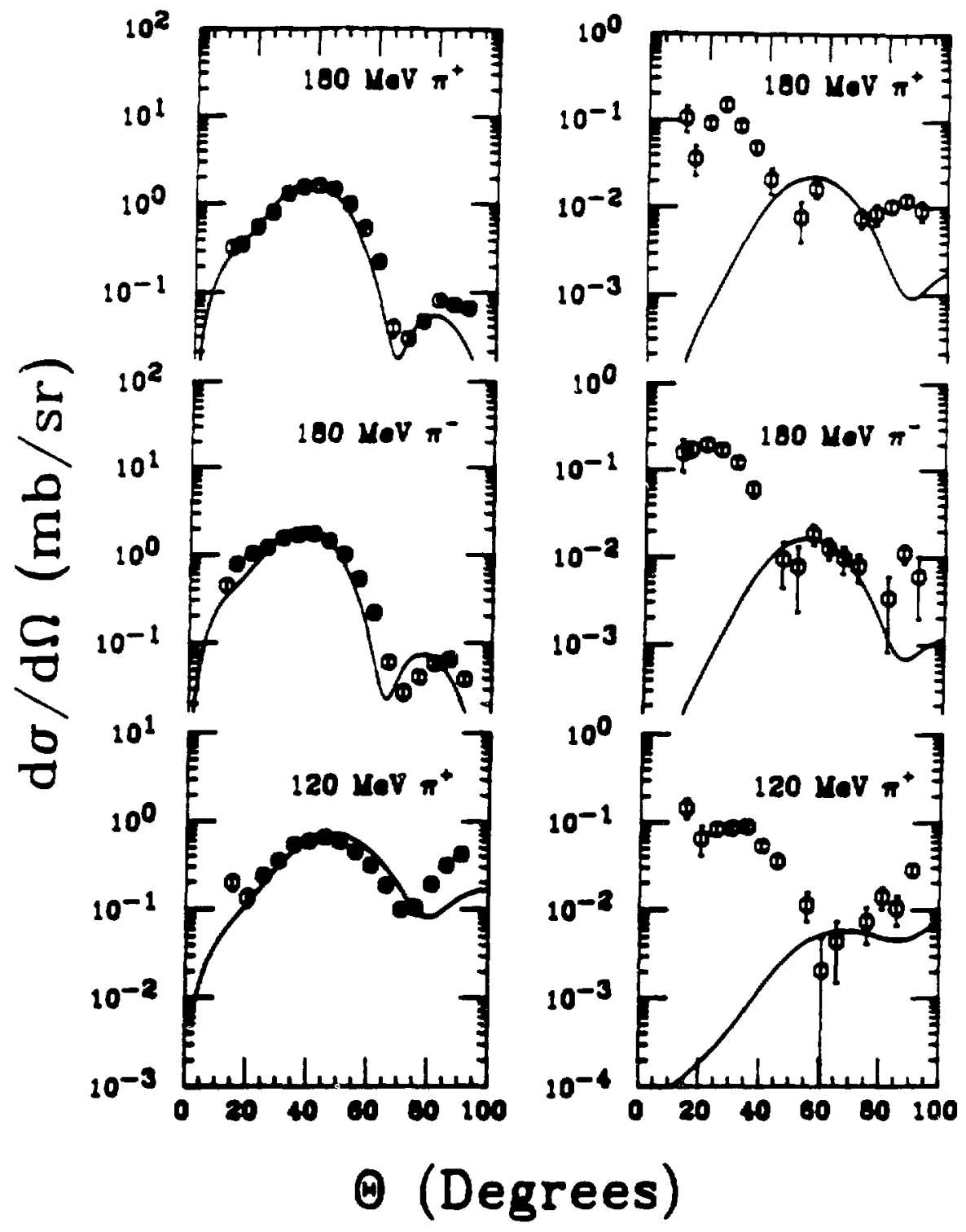

Ficure V-25.

Angular distributions for the $5.62-\mathrm{MeV} \mathrm{3}^{-}$(left column) and the $8.45-\mathrm{MeV}$ peak (right column). The top graph in each column is $180-\mathrm{MeV} \pi^{+}$, the middie $180-\mathrm{MeV} \pi^{-}$, and the bottom $120-\mathrm{MeV} \pi^{+}$. The curves are DWPI calculations. 


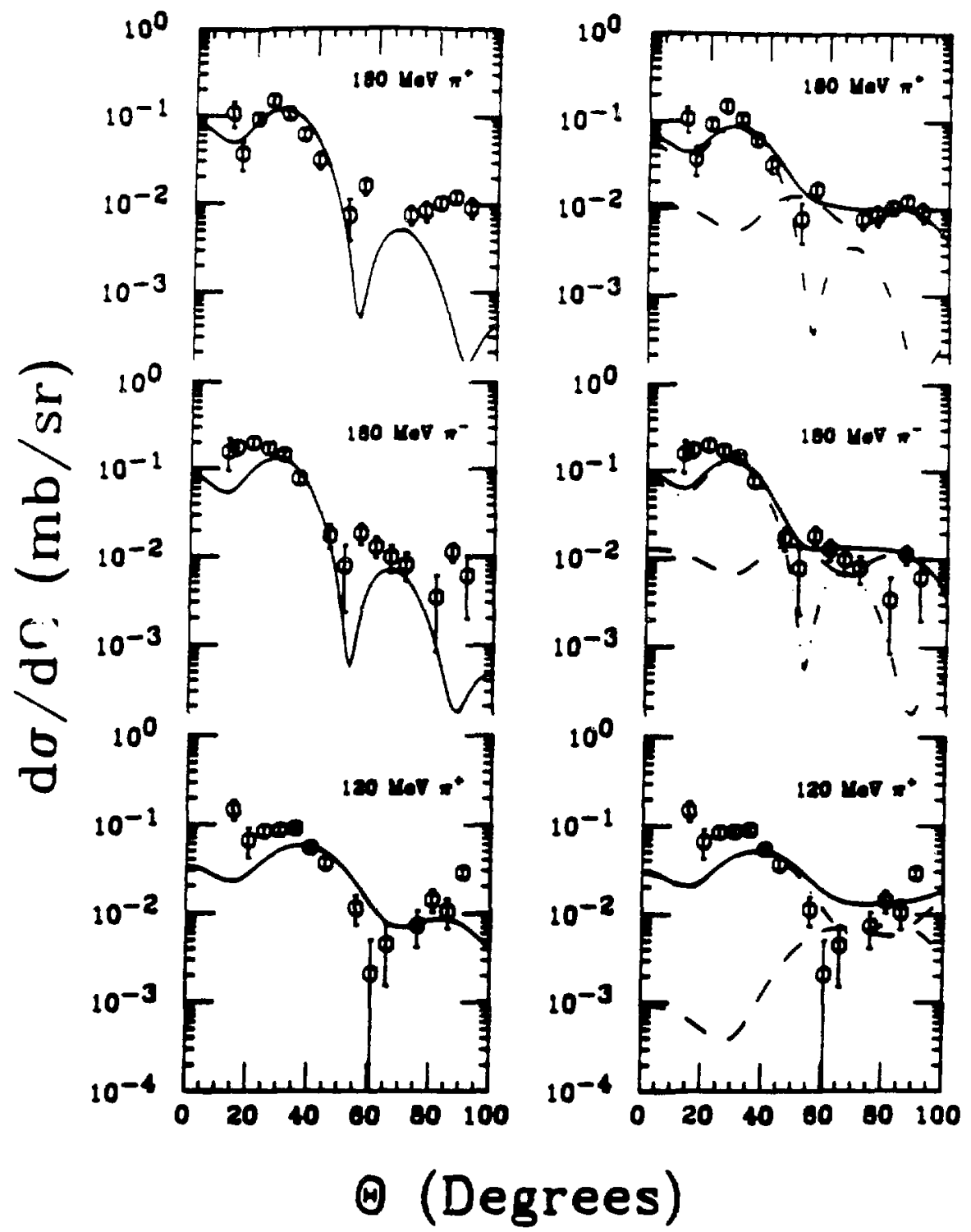

Figure V-26.

Angular distributions for the $8.45-\mathrm{MeV}$ peak. The top graph in each column is $180-\mathrm{MeV} \pi^{+}$, the middle $180 \mathrm{MeV} \pi^{-}$, and the bottom $120 \mathrm{MeV} \pi^{+}$. The solid curves in the left column and the dotdashed curves in the right column are DWPI calculations. In the right column the dashed curve are CCLA calculations and the solid curves are the incoherent sum of the CCLA and DWPI calculations. 


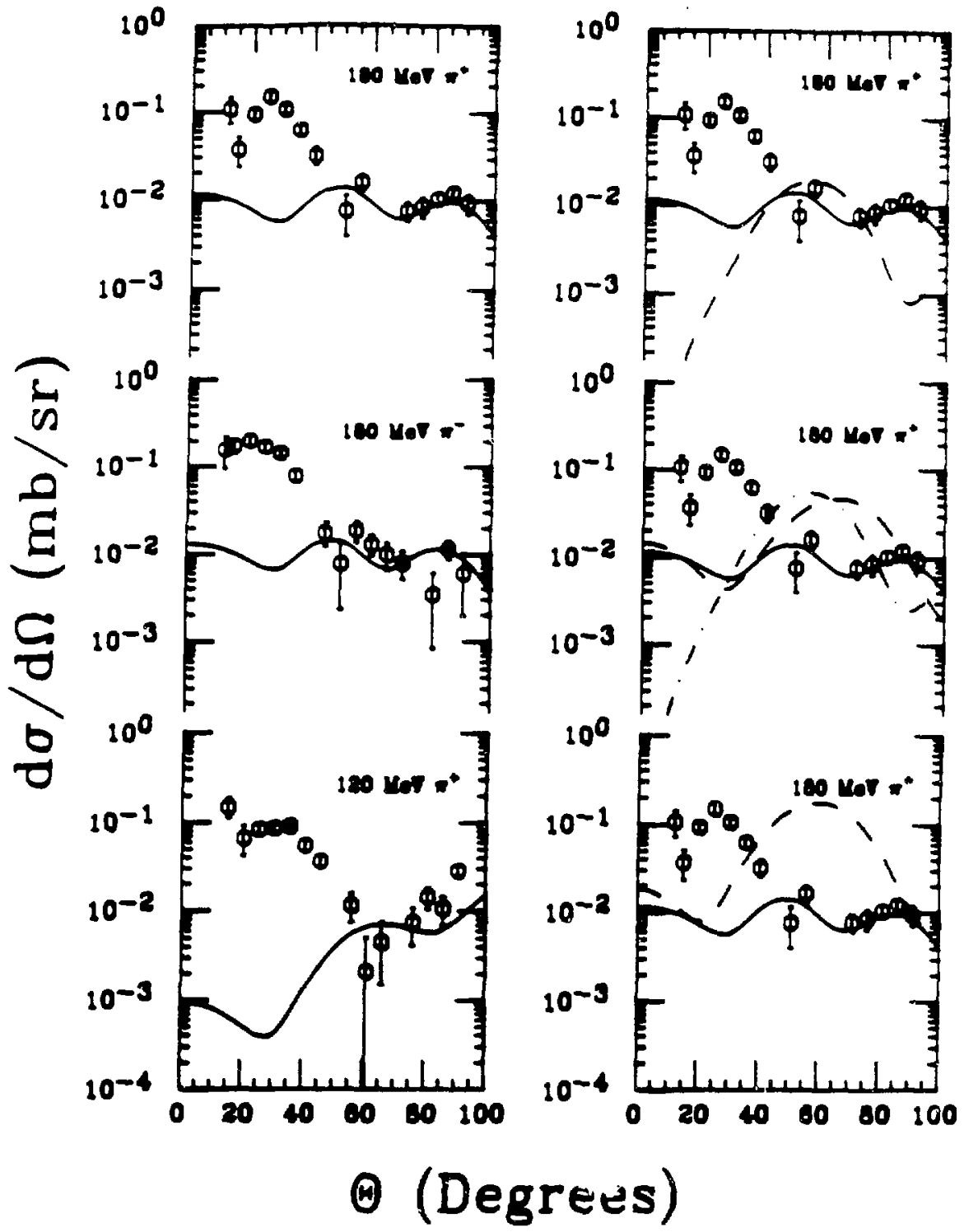

Figure V-27.

CCIA calculations to the $8.45-\mathrm{MeV} \mathrm{5}^{-}$. The top graph in left column is $180-$ $\mathrm{MeV} \pi^{+}$, the middle $180-\mathrm{MeV} \pi^{-}$, and the bottom $120 \mathrm{MeV} \pi^{+}$. In the right column all of the rraphs are $180-\mathrm{MeV} \pi^{+}$. The dashed curve in the right top graph is a DWPI calculation. In the right middle graph the dashed curves show the contributions of the different routes. In the right bottom graph the dashed curre shows the effect of changing the relative phace. 
added to the calculation, it can destructively interfere with the $3 M 1$ route and bring the calculated curve down to the level of the data. Increasing the E5 strength in the calculation can bring the coupled-channels curve down below the data. The E5 strength given in Table V-4 was chosen to reproduce the magnitude of the large angle data as shown in the left column of Figure V-27. With this strength to the $5^{-}$it is still possible to fit the angular distribution with a combination of the CCIA calculation and an $l=2$ DWIA calculation. The right column in Figure V-26 shows the incoherent sum (solid line) of the CCIA calculation (dashed line) and the $l=2$ DWIA calculation (dot-dashed line), implying the existence of a $2^{+}$state near the $8.45-\mathrm{MeV}^{-}$

\section{V-7. $K^{\pi}=0^{-}$band}

The three lowest states in this band are seen in this experiment, the $1^{-}$at 5.79 $\mathrm{MeV}$, the $3^{-}$at 7.16 - one of the strongest peaks in the spectrum - and the $5^{-}$at 10.26 $\mathrm{MeV}$. The $3^{-}$and $5^{-}$are shown in Figure V-28 with DWLA calculations and the extracted M's are given in Table V-3. The DWIA calculation reproduces the $3^{-}$angular distribution, as it does for all the strong peaks. The transition to the $1^{-}$will be discussed with the other $l=1$ transitions in Section V-9.

For the $5^{-}$the calculation puts the maximum and minimum at larger angles than the data. The $5^{-}$has a strong E2 $\gamma$ decay to the $3^{-},(\mathrm{AJ}-87)$, and the twostep calculation is strongest near the minimum of the data, although it is a factor of 4 less than the data as shown by the solid curve in the middle graph of the left column of Figure V-29. The dashed and dot-dashed curves are the $l=5$ strength which must be added coherently, constructively or destructively respectively, to the $3 \mathrm{M} 2$ calculation to reproduce the magnitude of the data. The left column shows the results of CCIA calculations adding the $3 \mathrm{M} 2$ and direct route 


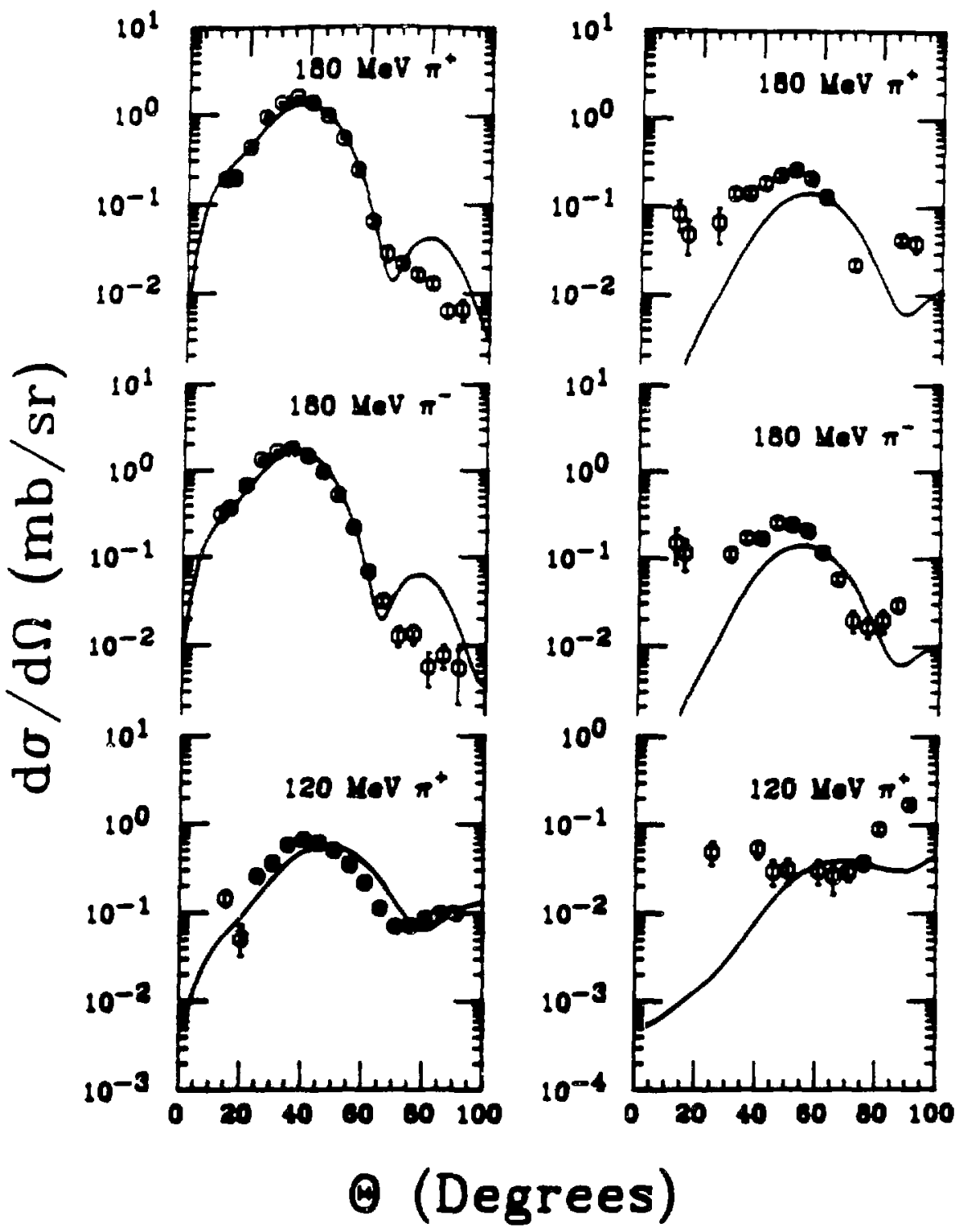

Figure V-28.

Angular distributione for the 7.16-MeV $3^{-}$(left column) and the $10.26-\mathrm{MeV}$ peak (right column). The top graph in each column is $180-\mathrm{MeV} \pi^{+}$, the middie $180-\mathrm{MeV} \pi^{-}$, and the bottom $120 \mathrm{MeV} \pi^{+}$. The curres are DWPI calculations. 


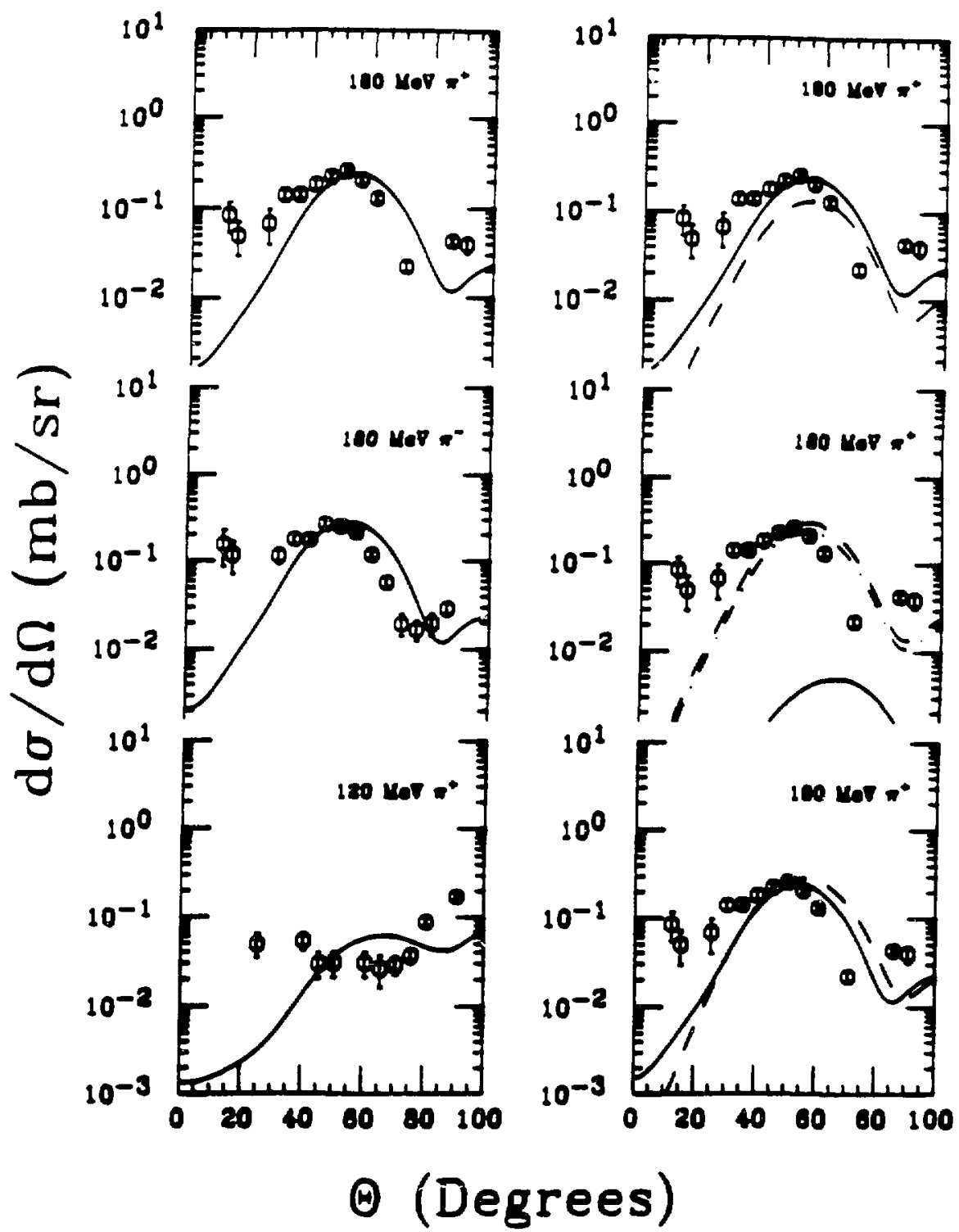

Figure V-29.

CCLA calculations to the $10.26-\mathrm{MeV} \mathrm{5}{ }^{-}$. The top graph in left column is 180 . $\mathrm{MeV} \pi^{+}$, the middle $180 \mathrm{MeV} \pi^{-}$, and the bottom $120-\mathrm{MeV} \pi^{+}$. In the right column all of the graphs are $180 \mathrm{MeV} \pi^{+}$. The dashed curve in the right top graph is a DWPI calculation. In the right middle graph the solid curve shows the contribution of the two-step route and the danhed the direct route depending on the phace. In the right bottom graph the dahed curve shows the effect of changing the relative phase. 
destructively. The bottom graph in the right column compares the results of adding the curves constructively (dashed line) and destructively (solid line). The coupling strengths used in these calculations are given in Table V-4. The CCIA calculations do not give any significant improvement in the 5 t to these data.

\section{V.8. $K^{\pi}=1^{-}$band}

Only one state from this bard was seen, the $3^{-}$at $10.4 \mathrm{MeV}$. Its angular digtribution is shown in Figure V-30 with DWIA calculations and the extracted gtrength is given in Table V-3. The peak is very weakly excited as is evident from the poor quality of the angular distribution, but given the quality of the data the calculation fits reasonably well. There are no $\gamma$ decays to or from this state listed in the compilation (AJ-87).

\section{V-9. $1^{-}$stated}

As discussed in Chapter 4 the $l=1$ transitions with $\Delta \mathrm{T}=0$ require special care as the $l=0$ transition at $6.73 \mathrm{MeV}$. Figure V-31 compares the transition density of Harakeh and Dieperink, (HA-81), with the standard collective transition density (solid curve). The two transition densities are obviously very different, even at large radii. There are two low-lying $1^{-}$states in ${ }^{20} \mathrm{Ne}$ seen in this experiment, at 5.79 $\mathrm{MeV}$ and $8.71 \mathrm{MeV}$. In addition there are six peaks above $10 \mathrm{MeV}$ whose angular distributions appear to contain some $l=1$ strength. Both the 5.79- and 8.71-MeV states have $\mathrm{T}=0$, so the standard transition density violates conservation of momentum. For the peaks above $10 \mathrm{MeV}$ the standard transition density can be used if $\Delta T=1$, i.e. a $T=1$ final state.

Six peaks which either should be or appear to be pure $l=1$ transitions are shown in Figures V-32 through V-34. The transition density of HA-81 generally 
TABLE V-5.

Transition strengths extracted with DWLA for peaks involving $l=0,1$ transitions.

$\begin{array}{llll}E_{\mathrm{x}} & l^{1)} & \mathrm{M}\left(\mathrm{efm}^{1+2}\right)^{2)} & \mathrm{R}_{\mathrm{tr}}^{2\}} \\ 5.79 & 1 & 6.2(0.4) \times 10^{0} & 6.7 \\ 6.73 & 0 & 5.27(0.04) & 5.95(0.07) \\ 8.71 & 1 & 8.1(0.6) \times 10^{0} & 6.7 \\ 11.22 & 1 & 3.3(0.4) \times 10^{0} & 6.7 \\ 11.48 & 1 & 2.9(1.3) \times 10^{0} & 6.7 \\ & 1+5 & <3.8(1.4) \times 10^{03} & 6.7 \\ & & <1.2(0.5) \times 10^{23} & - \\ & 1+6 & <3.8(0.9) \times 10^{03)} & 6.7 \\ & & <9 .(3 .) \times 10^{23)} & - \\ 13.72 & 1 & 3.5(0.5) \times 10^{0} & 6.7 \\ 14.88 & 1+5 & 3.9(0.5) \times 10^{04} & 6.7 \\ & & 2.2(0.3) \times 10^{24} & - \\ 15.59 & 1 & 5.4(0.9) \times 10^{0} & 6.7 \\ 17.27 & 1+4 & 2.7(0.3) \times 10^{-14} & 6.7 \\ & & 3.3(0.2) \times 10^{14} & - \\ & 1+5 & 2.8(0.4) \times 10^{-14} & 6.7 \\ & & 2.0(0.2) \times 10^{24} & -\end{array}$

1) $l$ is the angular momentum transfer used to fit the data. If more than one $l$ is listed then several $r_{s}$ were used to fit the data separately and the $M$ listed is for each fit. If $l_{1}+l_{2}$ is listed then $2 r_{3}$ were added incoherently to fit the data and the first $M$ listed is for $l_{1}$ and the second $M$, on the next line, is for $l_{2}$.

${ }^{2)} M$ and $R_{t r}$ are defined in the text for $l=0,1$ transitions. Except where noted all $\mathrm{M}^{\prime}$ 's were obtained by fitting $180-\mathrm{MeV} \pi^{+}$and $\pi^{-}$data simultaneously, with the constraint $M=M_{n}=M_{p}$. The number in parentheses is the statistical error of the fit.

${ }^{3)} \mathrm{M}$ was obtained by taking the maximum from either the $180-\mathrm{MeV} \pi^{+}$or $\pi^{-}$ data. The number in parentheses is the statistical error of the fit taken.

4) $\mathrm{M}$ was obtained by averaging the values for $180 \mathrm{MeV} \pi^{+}$and $\pi^{-}$. The number in parentheses is the statistical error of the fits. 


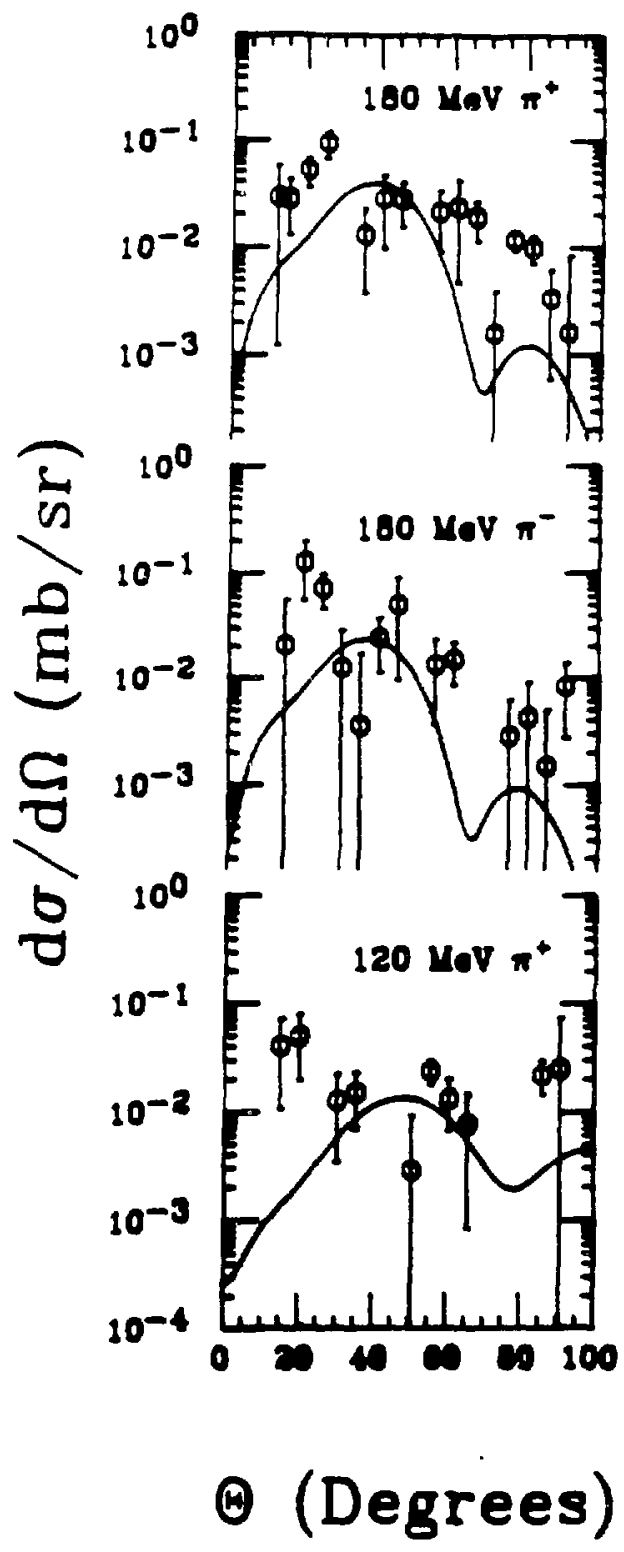

Firure V-30.

Angular distributions for the $10.41-\mathrm{MeV} 3^{-}$The top graph is $180-\mathrm{MeV} \pi^{+}$, the middle $180-\mathrm{MeV} \pi^{-}$, and the bottom $120-\mathrm{MeV} \pi^{+}$. The curres are DWPI calculations. 


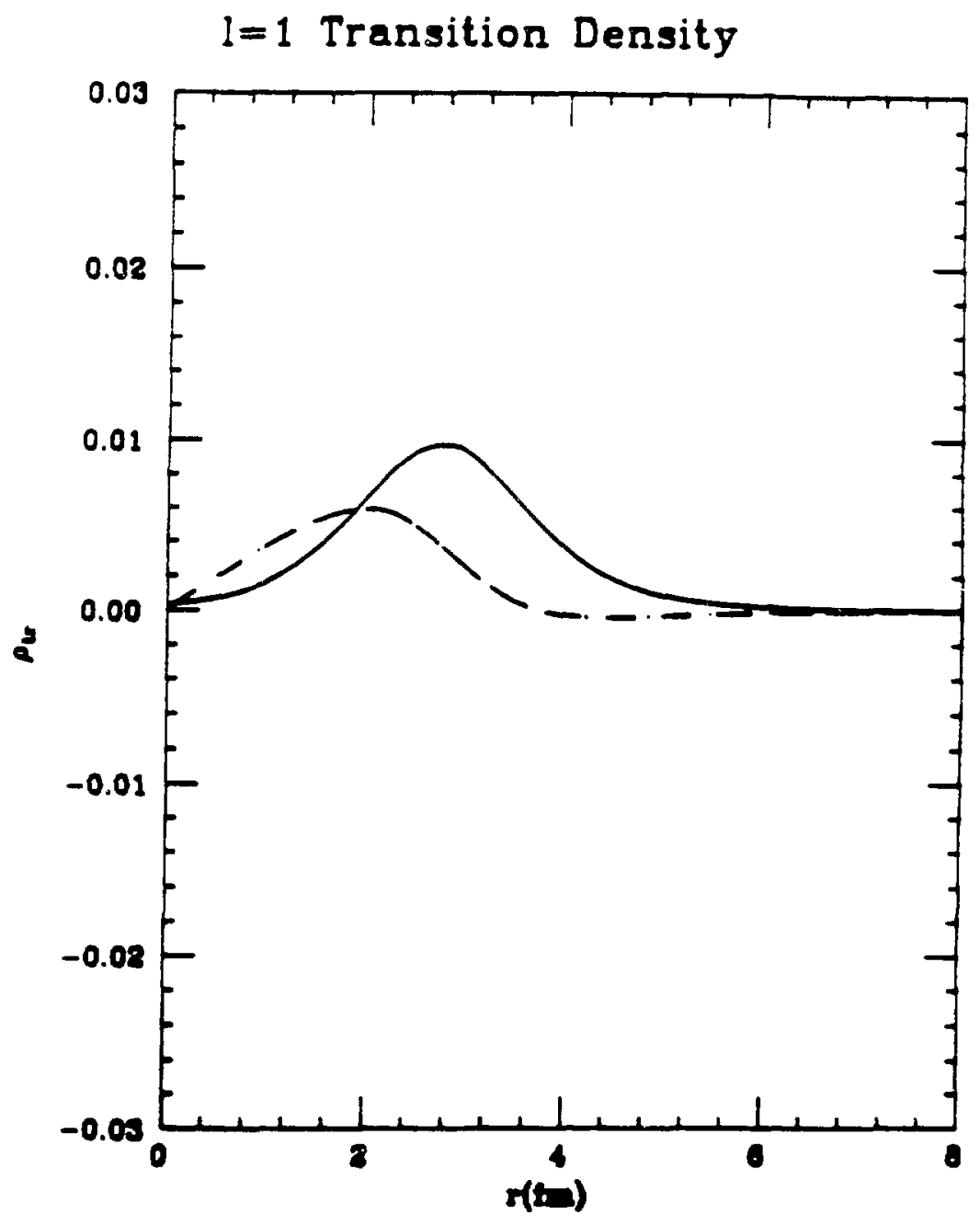

Figure V-31.

l=1 transition densitien used for transitions to $1^{-}$states. 


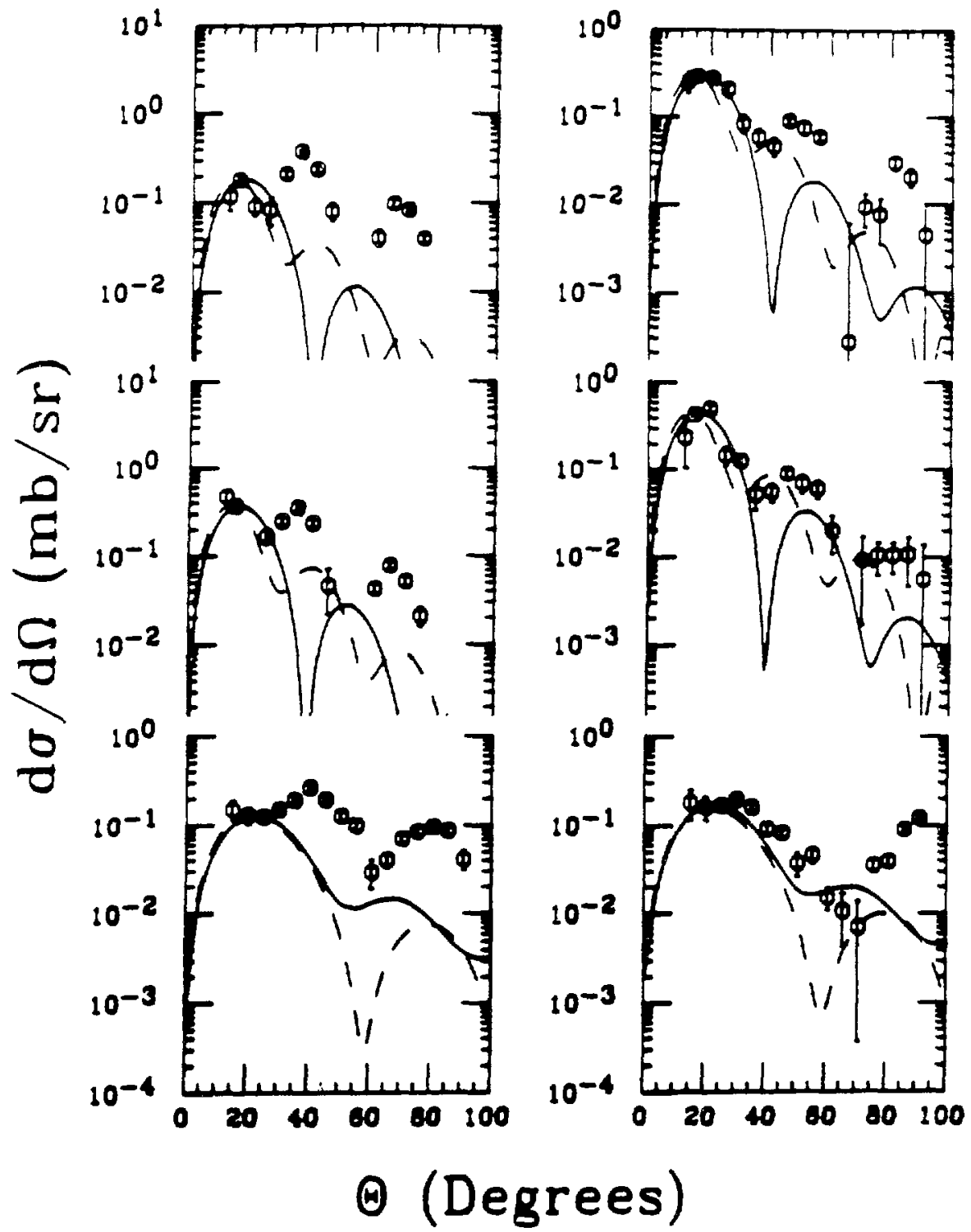

Figure V-32.

Angular distributions for the $5.79-\mathrm{MeV}^{-}$(left column) and the 8.71-MeV $1^{-}$ (right column). The top eraph in each column is $180-\mathrm{MeV} \pi^{+}$, the middle $180-\mathrm{MeV} \pi^{-}$, and the bottom $120-\mathrm{MeV} \pi^{+}$. The solid curves are DWPI calculations using the standard transition density, the dashed use the transition density of HA-81. 


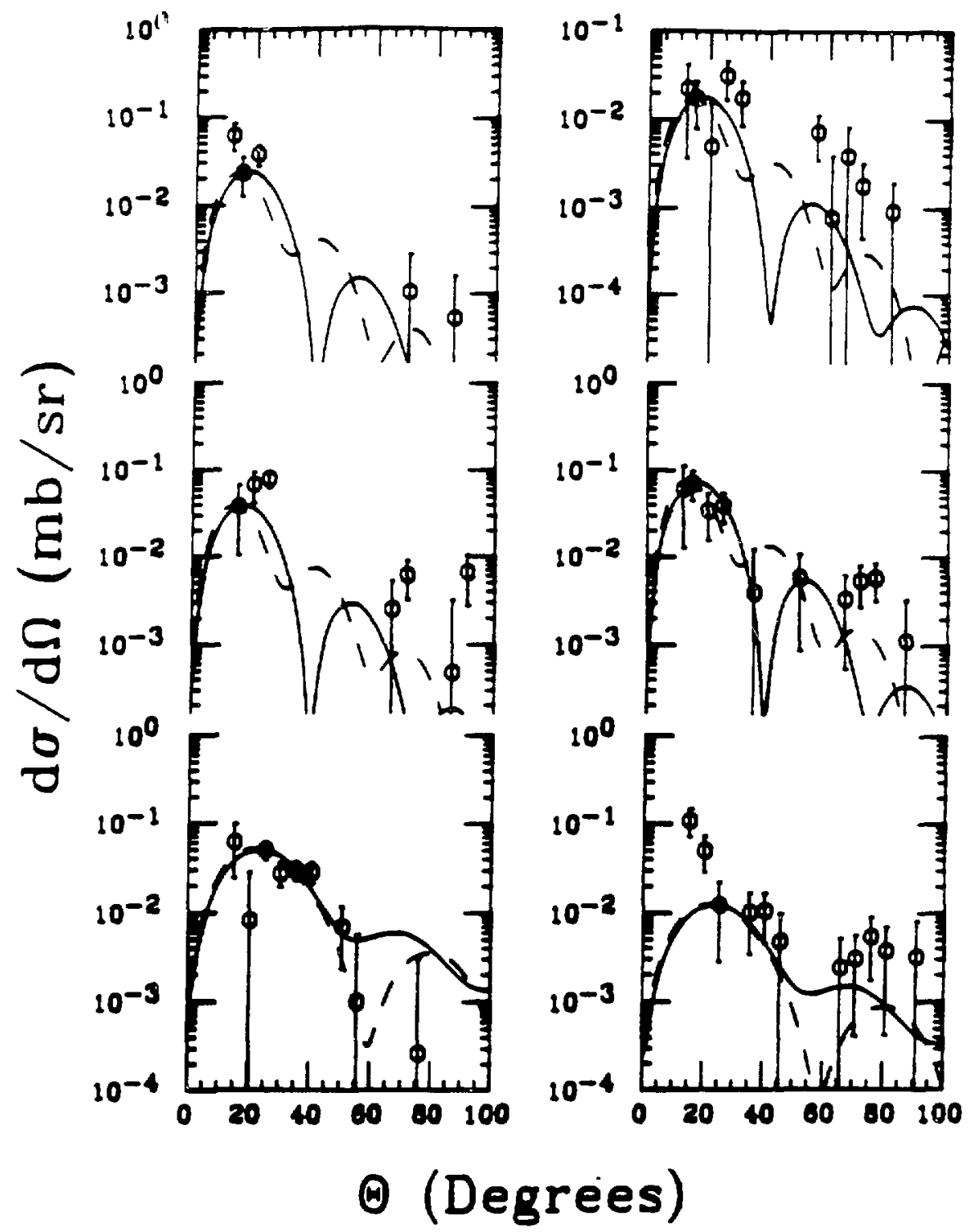

Figure V-33.

Angular distributions for the $11.22-\mathrm{MeV} \mathrm{1}^{-}$(left column) and the $11.48-\mathrm{MeV}$ $1^{-}$(right column). The top graph in each column is $180-\mathrm{MeV} \pi^{+}$, the middle $180-\mathrm{MeV} \pi^{-}$, and the bottom $120-\mathrm{MeV} \pi^{+}$. The solid curve are DWPI calculations using the standard transition density, the danhed use the transition density of HA-81. 


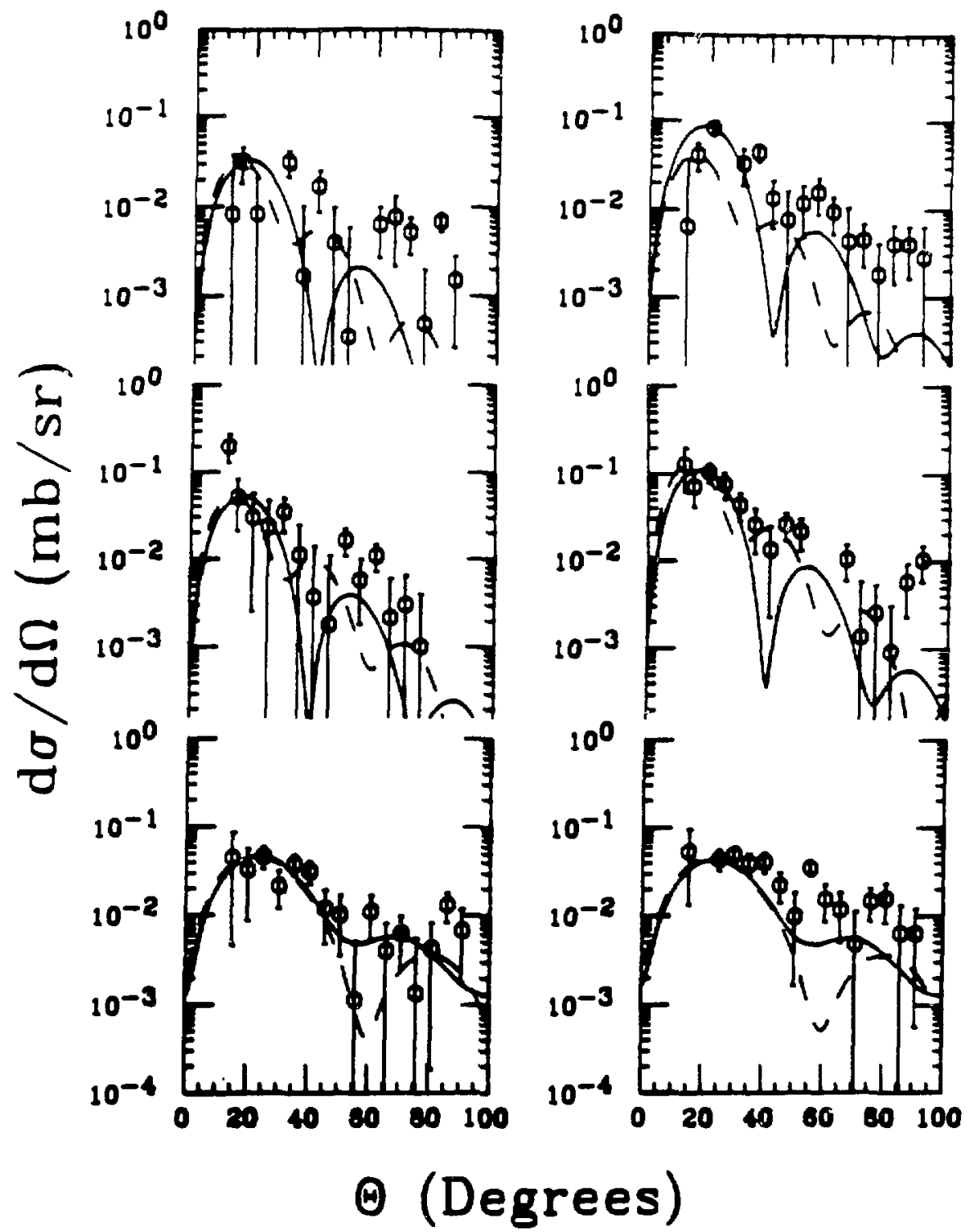

Figure V-34.

Angular distributions for the $13.72-\mathrm{MeV} \mathrm{1}^{-}$(left column) and the $15.50-\mathrm{MeV}$ $1^{-}$(right culumn). The top graph in each column is $180-\mathrm{MeV} \pi^{+}$, the middle $180-\mathrm{MeV} \pi^{-}$, and the bottom $120-\mathrm{MeV} \pi^{+}$. The solid curres are DWPI calculations using the standard transition density, the dashed use the transition density of HA-81. 
gives a better fit, although it generally gives the first minimum at a smaller angle than the data. Because the transition density has been chosen such that the normal definition of $M$ is identically zero, the definition of $M$ and $R_{t r}$ used for $l=0$ transitions will be used for the $l=1$ transitions,

$$
M=\left\langle r^{2}\right\rangle_{t r} \text { and } R_{t r}^{2}=\frac{\left\langle r^{4}\right\rangle_{t r}}{\left\langle r^{2}\right\rangle_{t r}}
$$

Table V-5 contains the values of $M$ and $R_{t r}$ extracted for $l=0$ and $l=1$ transitions.

Three peaks appear to be doublets, i.e. their angular distributions appear to be the result of two is adding incoherently. The peak at $11.48 \mathrm{MeV}$ appears to be a combination of either $l=1+4$ or $l=1+5$, shown in Figures V-35 and V-36. The $180-\mathrm{MeV} \pi^{+}$data seem to prefer the transition density of HA-81, while the $\pi^{-}$ data are better fitted by the standard transition density. The peak at $14.88 \mathrm{MeV}$ is well fitted assuming the peak is a $1^{-}, 5^{-}$doublet, using the transition density of HA-81, as shown in Figure V-37. The peak at $17.27 \mathrm{MeV}$, the highest excitation peak seen in this experiment, is fitted by either $l=1+4$ or $l=1+5$, and again some angular distributions are better fitted by the standard transition density and some by the transition density of HA-81, as seen in Figures V-38 and V-39.

The $8.71-\mathrm{MeV}^{-}$is the lowest-lying state in ${ }^{20} \mathrm{Ne}$ not placed in a rotational band, (AJ-87). This state is known to $\gamma$ decay to the ground state and the first $2^{+}$, (AJ-87). The strengths of both decays are measured, (AJ-87), but the $\gamma$ decay to the $2^{+}$is an $\mathrm{E} 1$ transition, which is generally weaker than an E3 in pion scattering. Since there is a strong coupling between the $1^{-}$and $2^{+}$, a two-step calculation using the $0^{+}-2^{+}-1^{-}$route, assuming the $2^{+}-1^{-}$transition is E3, was performed varying the strength of the E3 coupling. The results of this calculation are shown in the left column of Figure V-40. The two-step calculation fits the data as well as the DWLA calculation using either transition density, shown in the right column of 


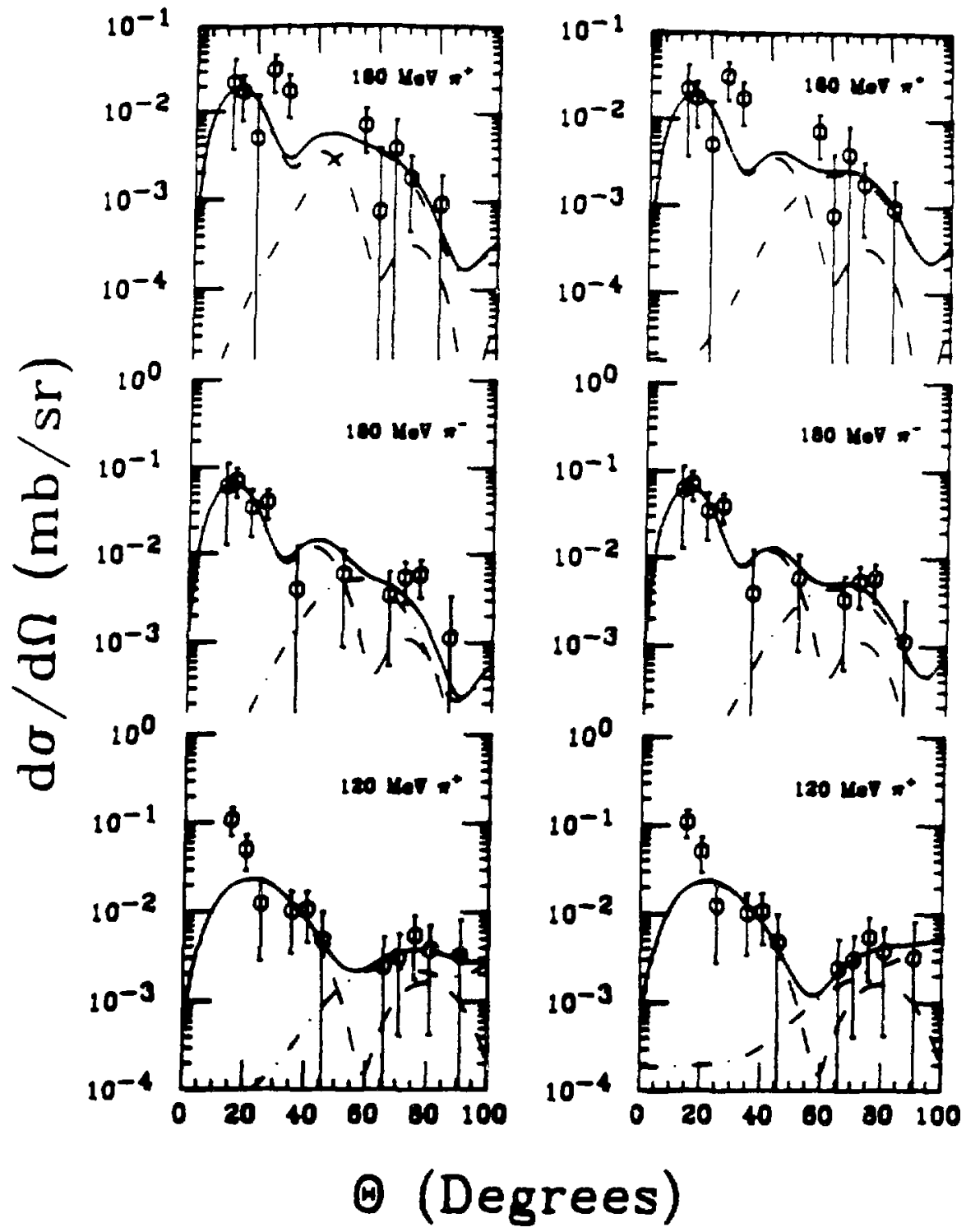

Figure V-35.

Angular distributions for the $11.48 \mathrm{MeV}$ peak. The top graph in each column is $180-\mathrm{MeV} \pi^{+}$, the middle $180-\mathrm{MeV} \pi^{-}$, and the bottom $120-\mathrm{MeV} \pi^{+}$. The left column asoumes the pesk is $a 1^{-}$state and $a 4^{+}$state. The right column asoumes the peak is a $1^{-}$state and a $5^{-}$state. The curres are DWPI calculations. 


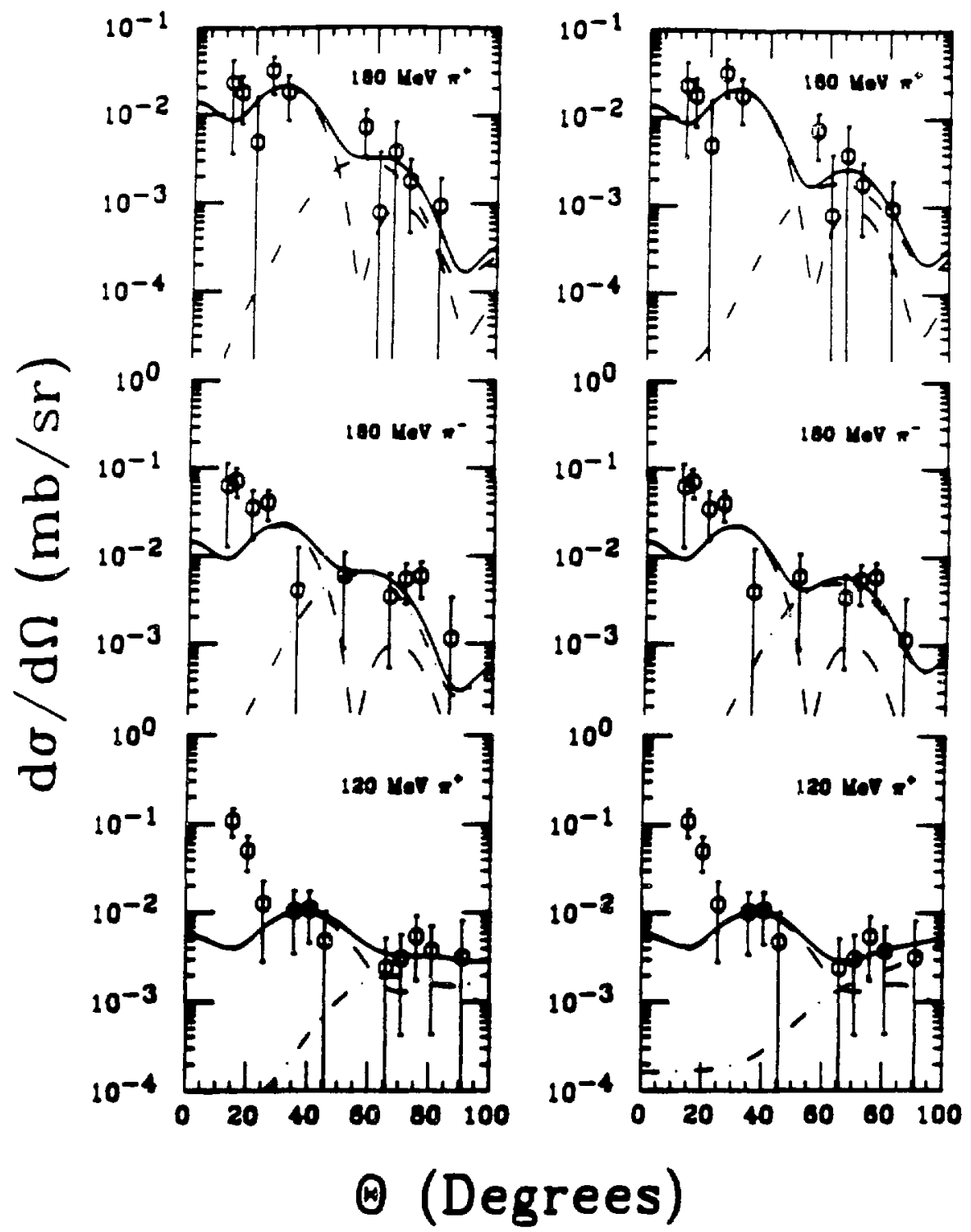

Figure V-36.

Same as Figure V-35 except the $E 1$ cal:ulations use the transition denoity of HA-81. 


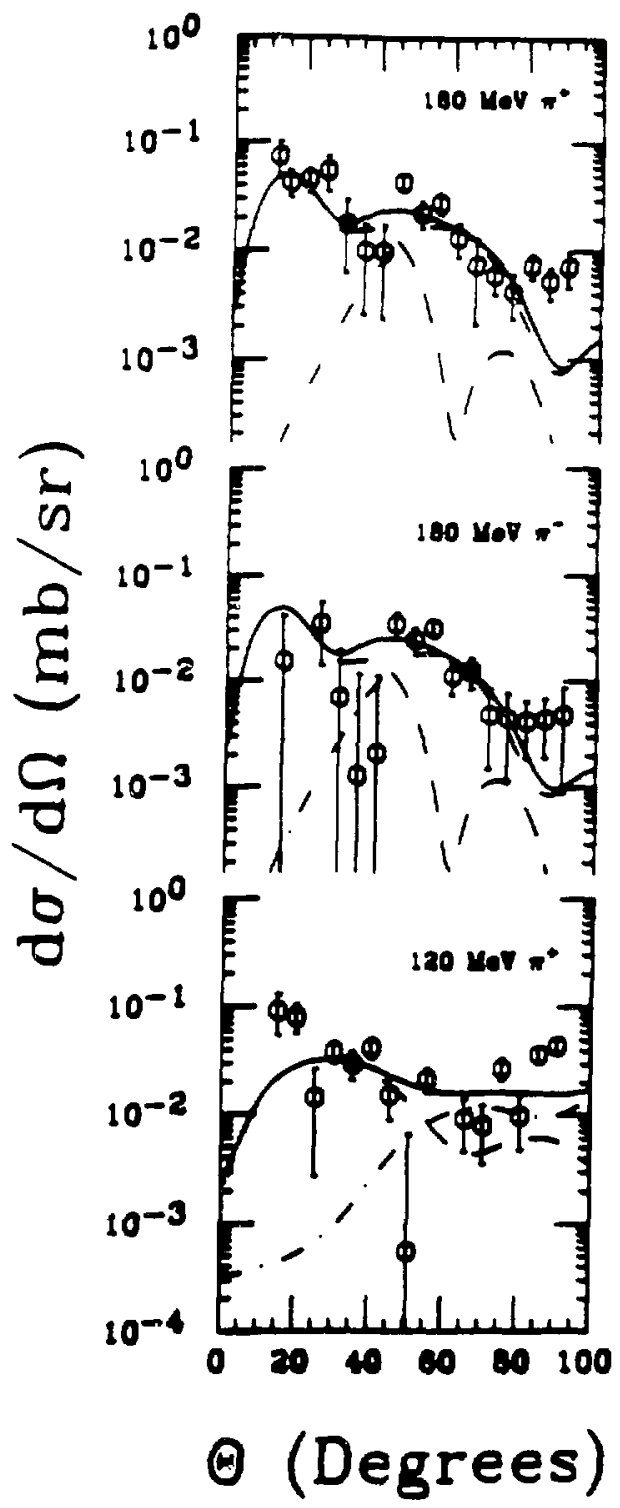

Figure V-37.

Angular distributions for the $14.88 \mathrm{MeV}$ peak. The top graph is $180-\mathrm{MeV}$ $\pi^{+}$, the middle $180 \mathrm{MeV} \pi^{-}$, and the bottom $120 \mathrm{MeV} \pi^{+}$. The curves are DWPI calculation. 


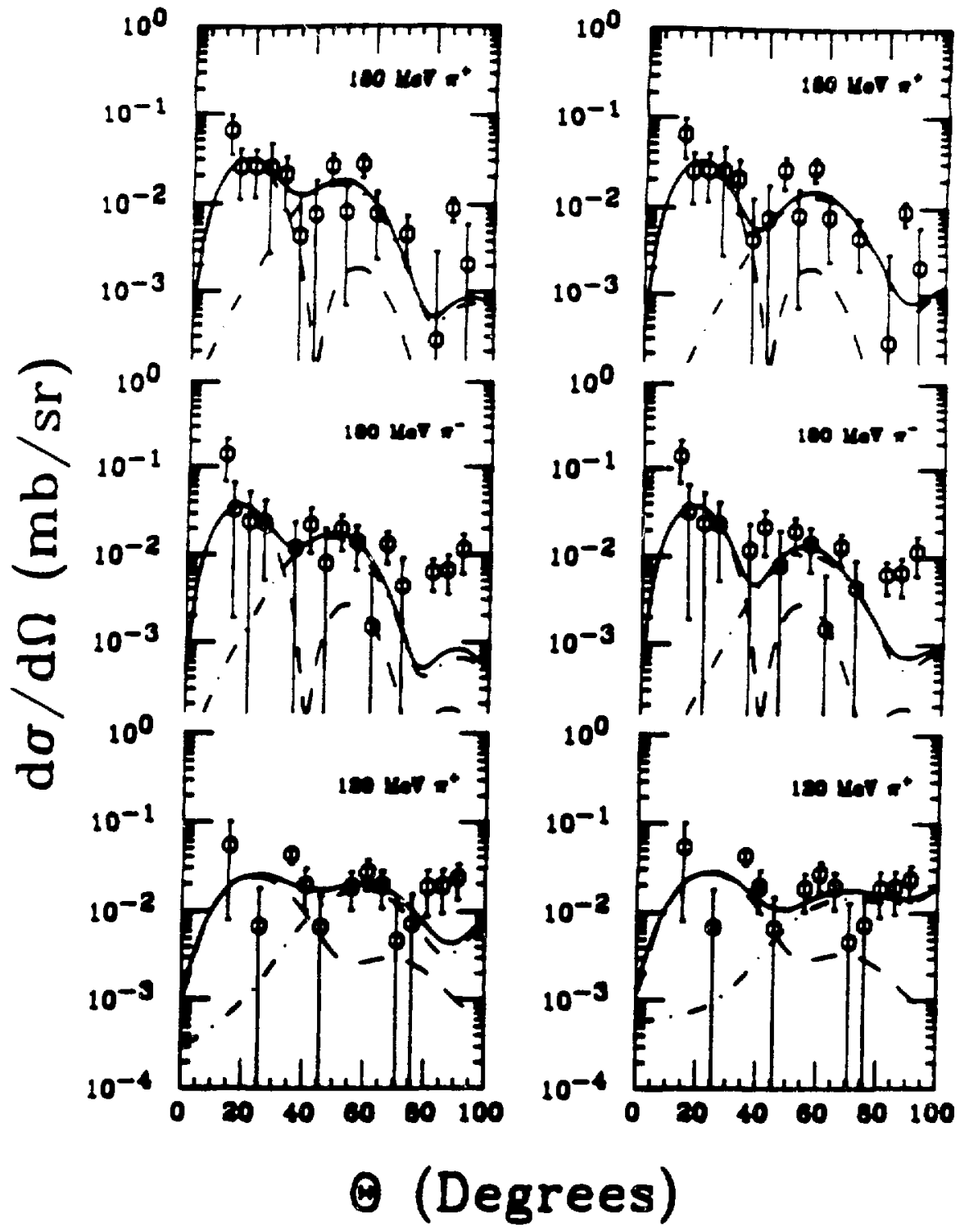

Figure V-38.

Angular distributions for the 17.27-MeV peak. The top araph in each column is $180-\mathrm{MeV} \pi^{+}$, the middle $180-\mathrm{MeV} \pi^{-}$, and the bottom $120-\mathrm{MeV} \pi^{+}$. The left column asoumes the peak is $a 1^{-}$state and $\triangle 4^{+}$state. The right column asoumes the peak is a $1^{-}$state and a $5^{-}$state. The curves are DWPI calculations. 

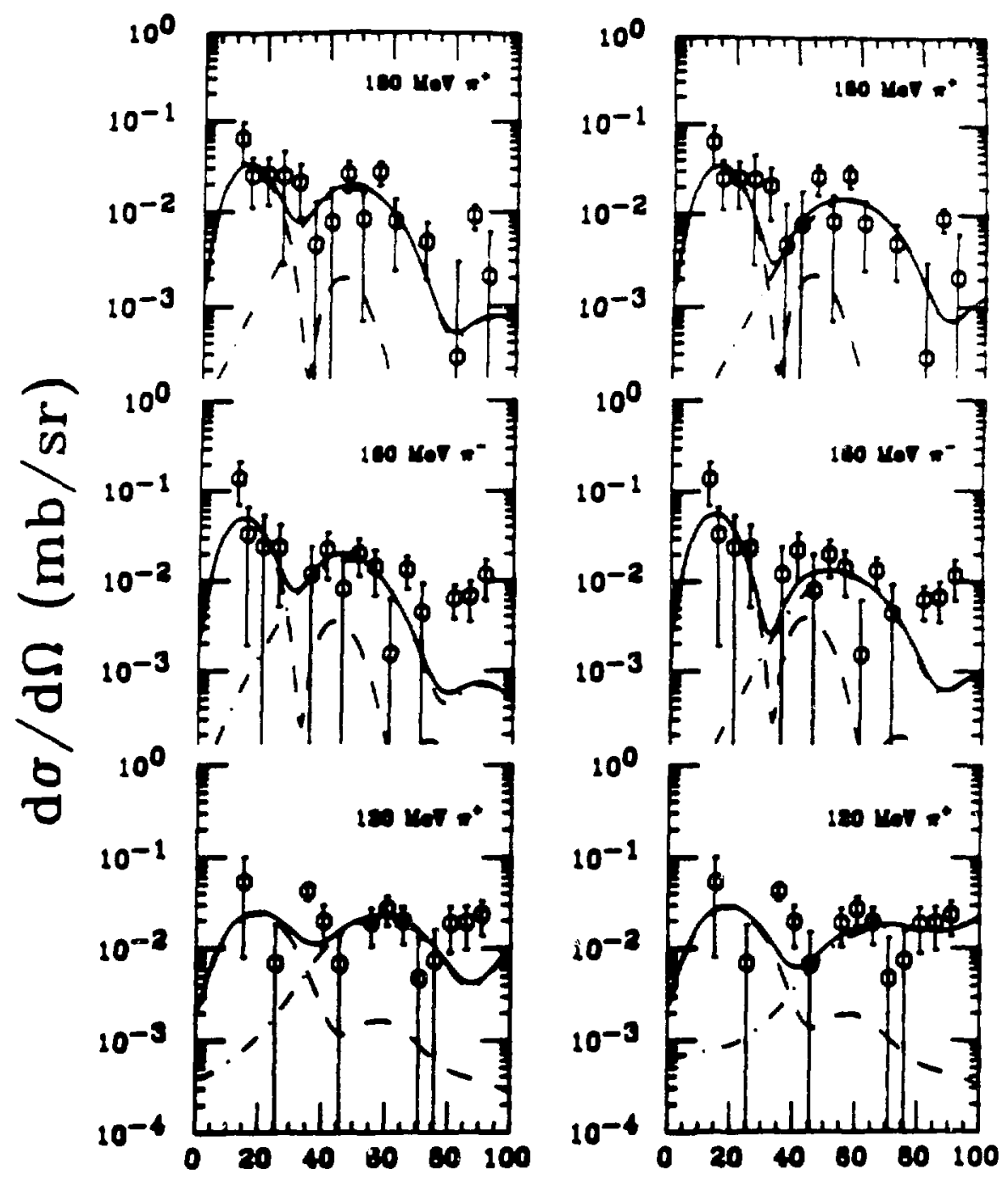

()ㅜ (Degrees)

Pigure V-39.

Same 20 Figure $V-38$ except the $l=1$ calculations use the transition density of HA-81. 


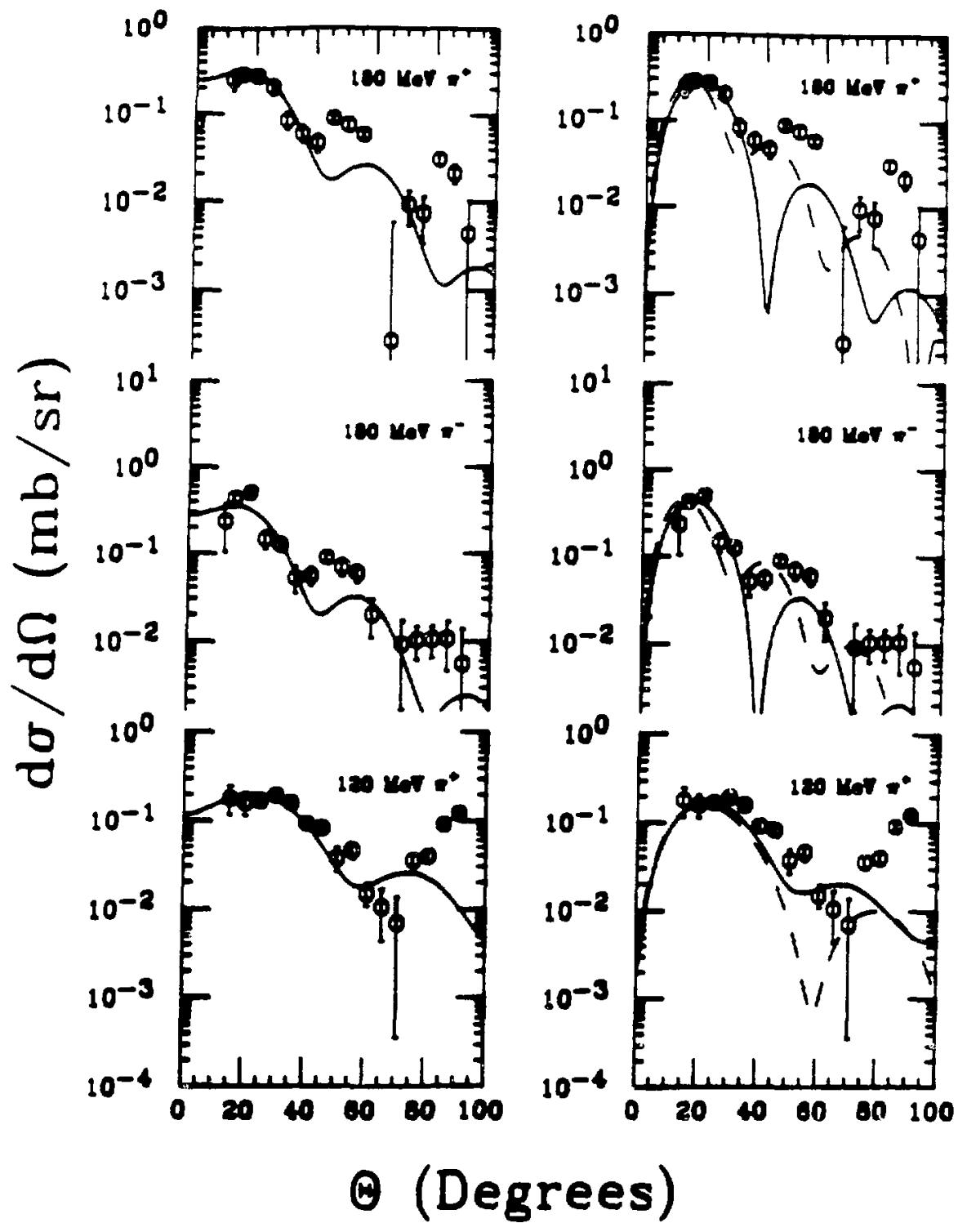

Figure V-40.

Angular distributions for the 8.71- $\mathrm{MeV} \mathrm{1}^{-}$. The top graph in each column is $180-\mathrm{MeV} \pi^{+}$, the middle $180-\mathrm{MeV} \pi^{-}$, and the bottom $120-\mathrm{MeV} \pi^{+}$. The curves in the left column are two-step calculations. The curves in the right column are DWPI calculations using the standard collective transition density (solid line) and the transition density of HA-81 (dashed line). 
Figure V-40.

The $5.79-\mathrm{MeV}$ angular distribution is poorly fitted by the DWIA calculations, using either transition density. This state is known to have a strong decay to the 1.63- $\mathrm{MeV} 2^{+}$, and the $7.16-\mathrm{MeV} 3^{-}$has a strong E2 decay to it. Therefore there are two two-step routes which can strongly excite this state, $0^{+}-3^{-}-1^{-}$and $0^{+}-2^{+}-1^{-}$. Again, because $\mathrm{E} 1$ transitions tend to be much weaker than E3 transitions an $l=3$ coupling was used for the $2^{+}-1^{-}$transition. It was assumed to have the same strength as the $0^{+} \rightarrow 3^{-}$transition, which is a similar cross-band transition. The couplings used are shown in Figure V-41, including a direct route which used the transition density of HA-81, and whose strength was varied to reproduce the magnitude of the data. Figures V-42 through V-44 show the results of these calculations. There are four choices of the relative phases of each of the ro'stes, one of which fits the $180-\mathrm{MeV}$ data very well as shown in Figure V-42. The different choices of the relative phases give very different curves, as seen in Figure V-43. Figure V-44 shows the relative contribution of each route to the transition, the $0^{+}-2^{+}-1^{-}$route gives the dashed curve, the $0^{+} \rightarrow 3^{-}-1^{-}$route gives the alternating long and short dashed curve, and the direct route is given by the dot-dashed curve. All three routes contribute roughly equally to the transition.

\section{V-10. Microscopic calculations}

Two shell-model calculations of the structure of ${ }^{20} \mathrm{Ne}$ were described in Chapter 3. They have been used to generate transition densities for microscopic calculations of the angular distributions using the computer codes PIPIT (EI-76b) and HL (LE-80b), described in Chapter 4. Isoscalar and isovector effective charges can be extracted by comparing the magnitude of the data and the calculations to $T=0$ and $T=1$ states respectively. Table $V-6$ contains the effective charges 


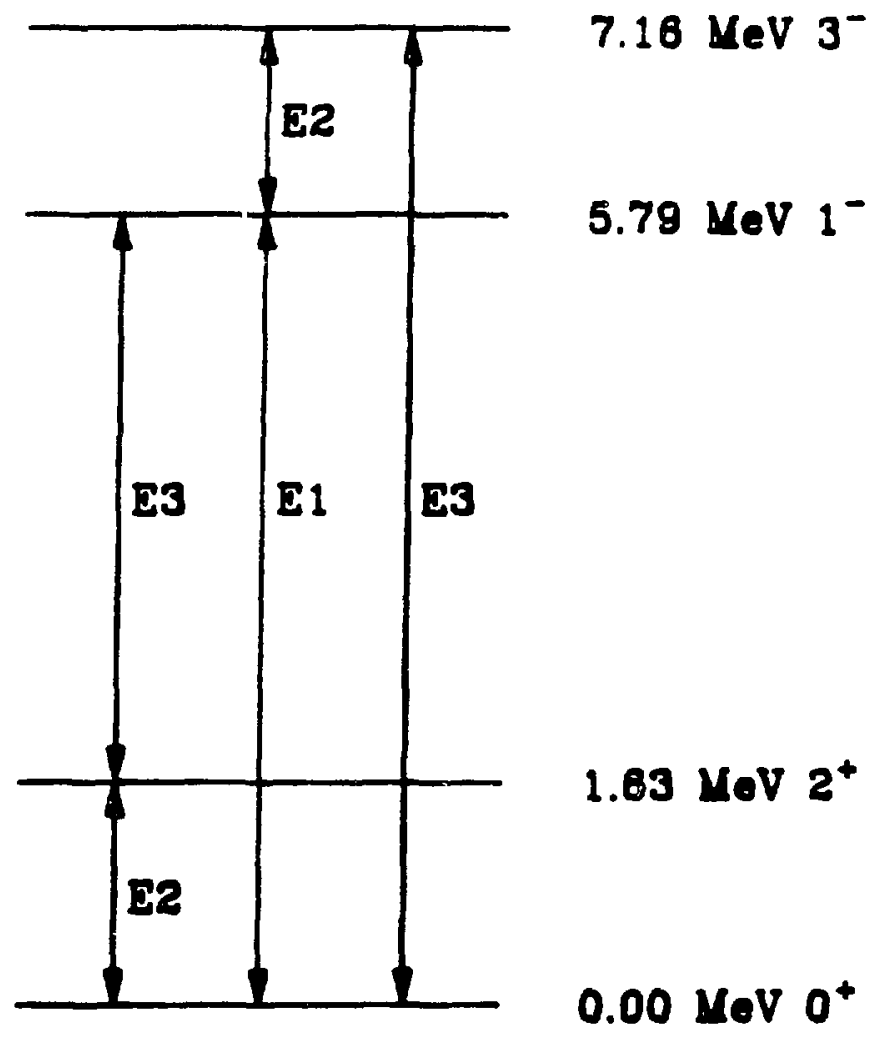

Figure V-41.

Couplings used in the CCLA calculations for the $5.79-\mathrm{MeV}^{-}$state. 


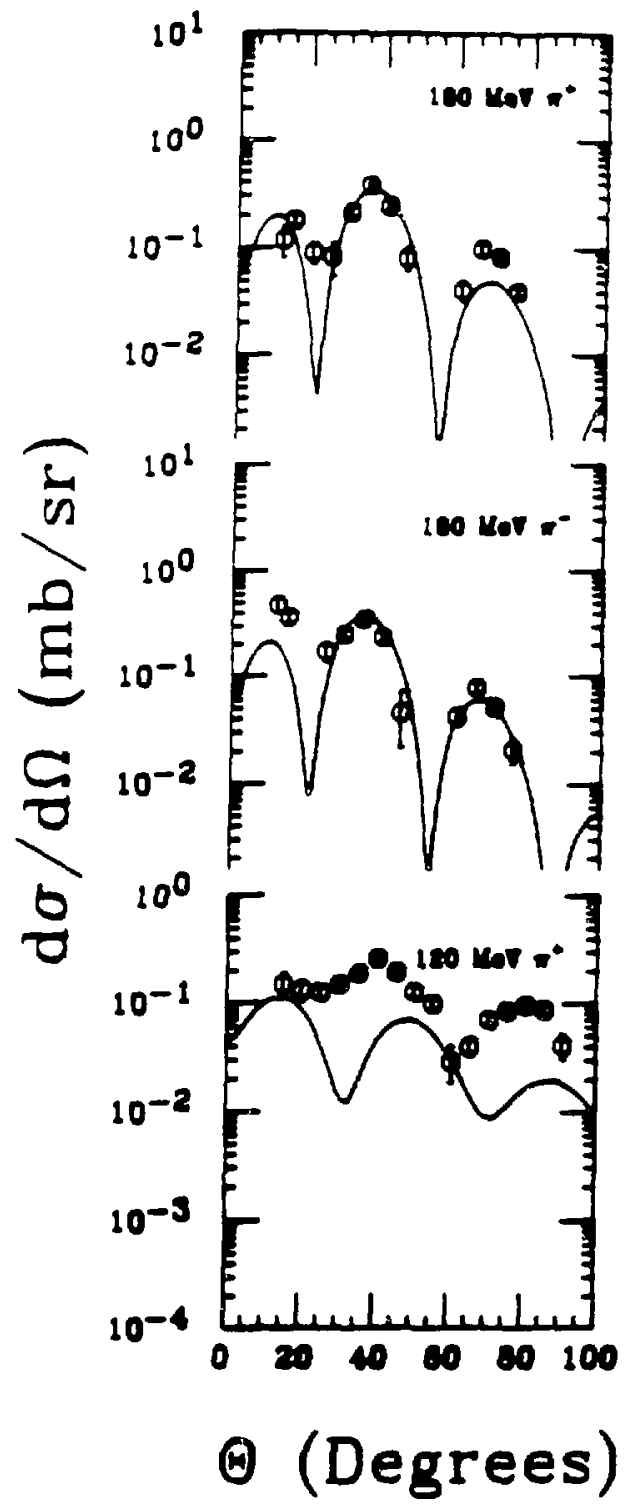

Figure V-42.

CCLA calculations to the $5.79-\mathrm{MeV}^{-}$. The top graph is $180-\mathrm{MeV}^{+}$, the middle $180-\mathrm{MeV} \pi^{-}$, and the bottom $120-\mathrm{MeV} \pi^{+}$. 


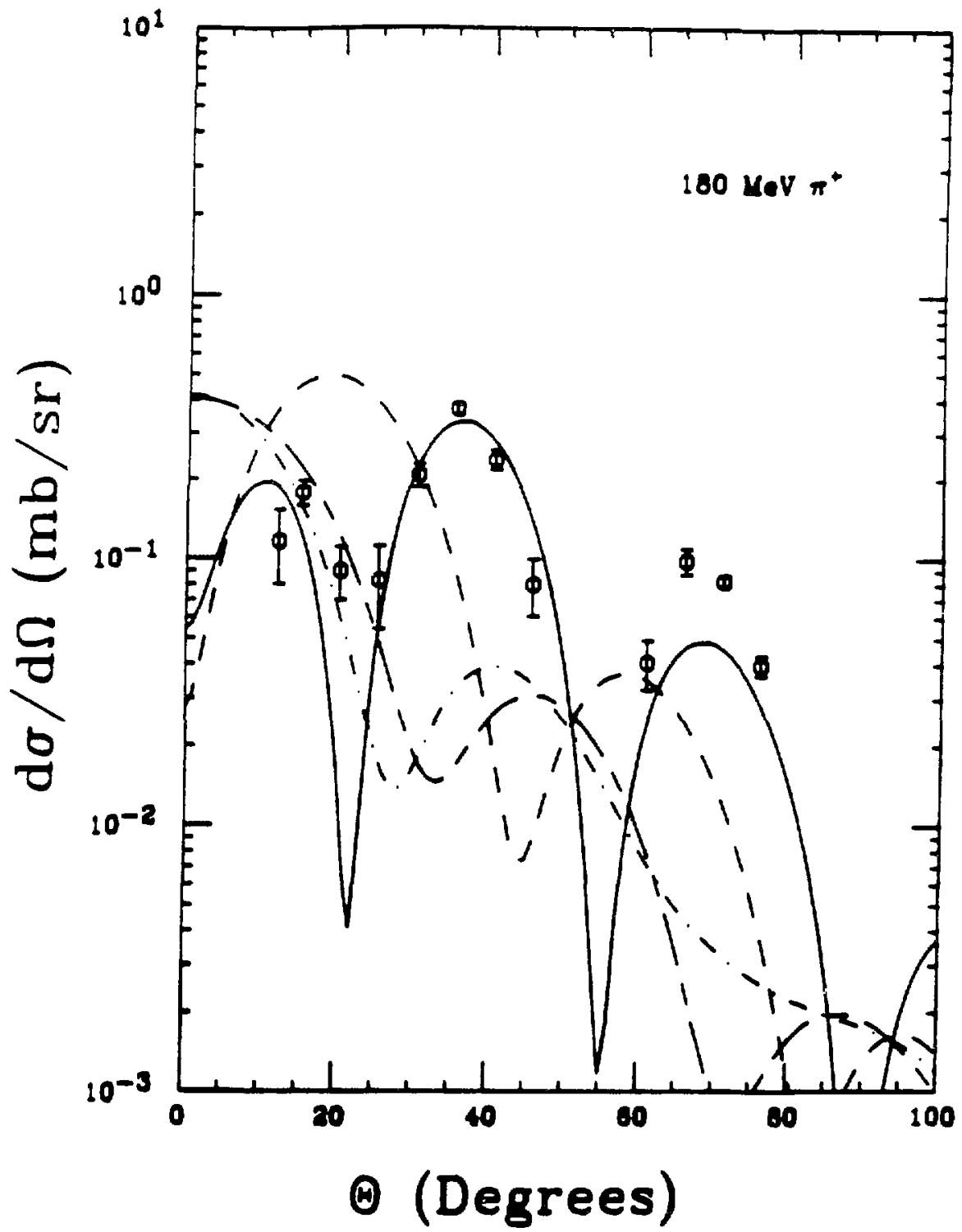

Figure V-43.

CCLA calculations to the $5.79 \mathrm{MeV} 1^{-}$. The curves shon the effect of changing the relative phase between the routes. 


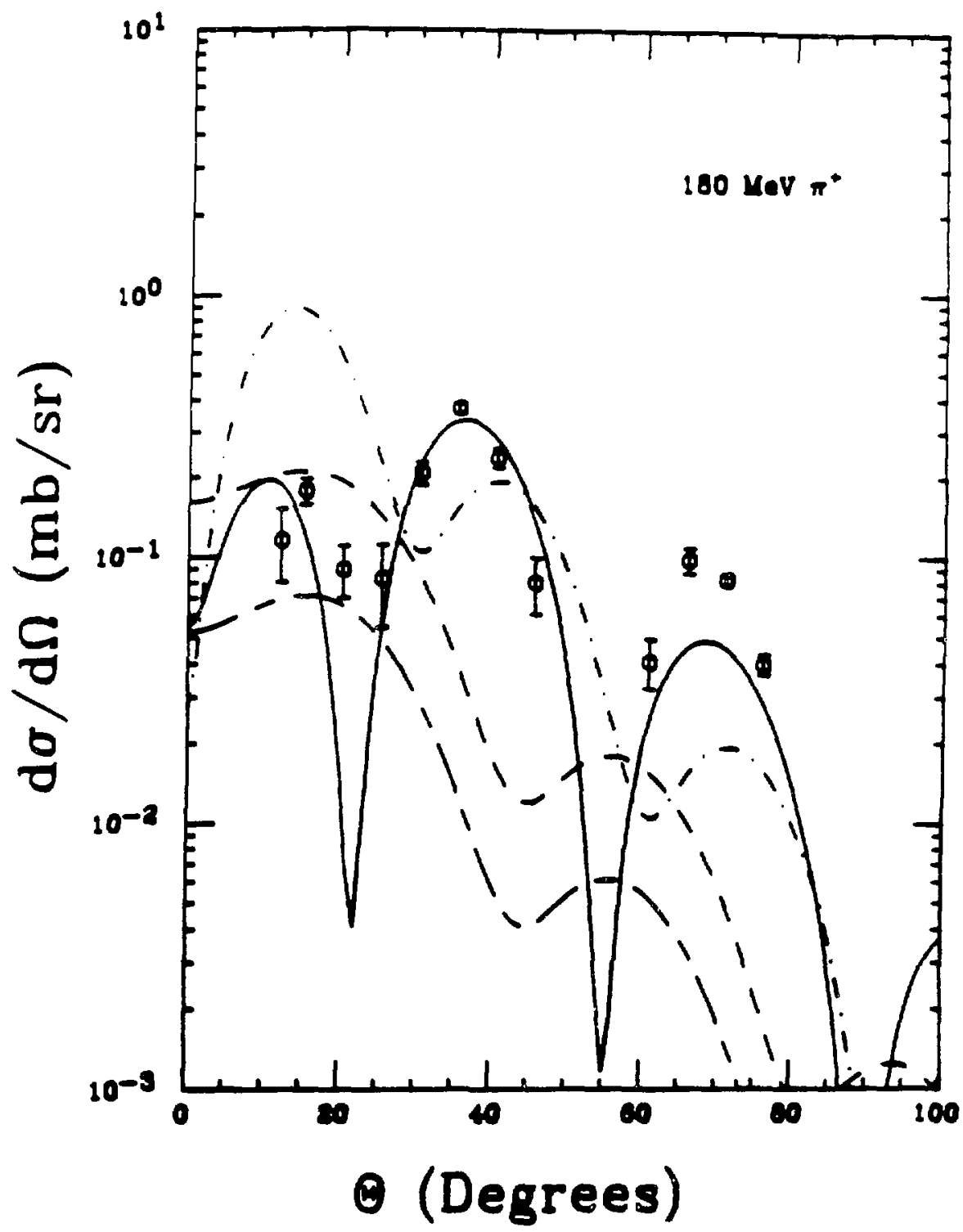

Fisure V-44.

CCLA calculations to the $5.79 \mathrm{MoV} 1^{-}$. The curve show the contribution of each route. 


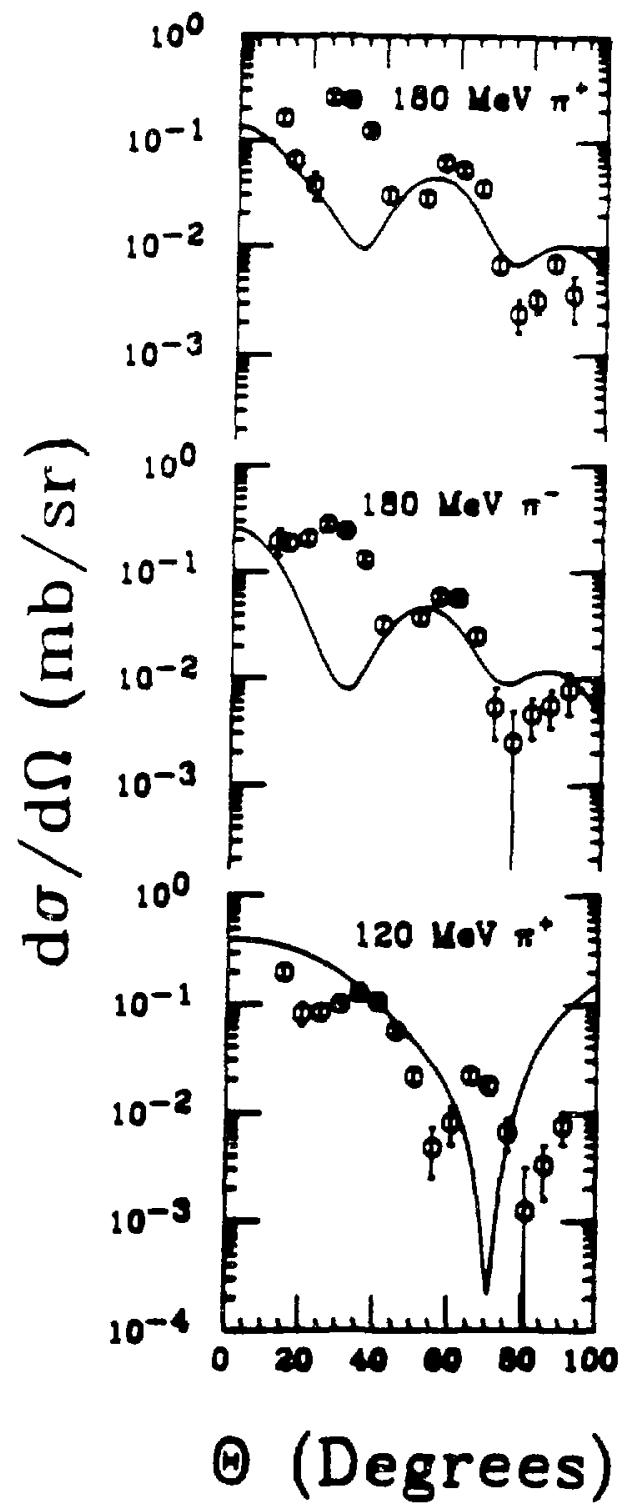

Figure V-45.

Angular distributions for the $6.73-\mathrm{MeV}^{+}$. The top graph is $180-\mathrm{MeV}^{+}$, the middle $180-\mathrm{MeV} \pi^{-}$, and the bottom $120 \mathrm{MeV} \pi^{+}$. The curves are DWIA calculations using microscopic transition densities from the (sd) shell-model calculation. 


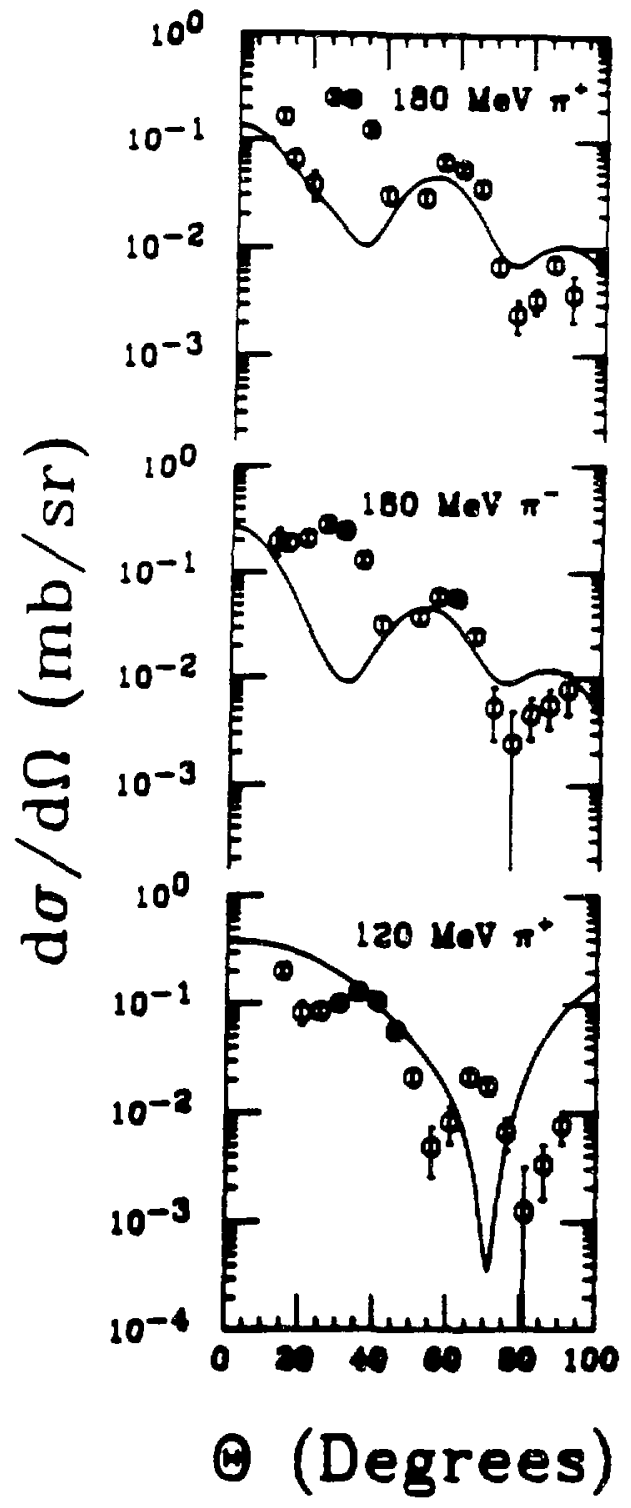

Figure V-46.

Aagular distributions for the 6.73- $\mathrm{MeV} \mathrm{O}^{+}$. The top graph is $180-\mathrm{MeV}^{+}$, the middle $180-\mathrm{MeV} \pi^{-}$, and the bottom $120-\mathrm{MeV} \pi^{+}$. The curves are DWIA calculations using microscopic transition densities from the ZBM shell-model calculation. 
extracted from these data. The first $\mathrm{T}=1$ state in ${ }^{20} \mathrm{Ne}$ comes at $10.27 \mathrm{MeV}$ and is a $2^{+}$. The angular distribution for the nearest peak, $10.26 \mathrm{MeV}$, has no apparent contribution from this state. Some of the higher excitation peaks may correspond to $T=1$ states. These states are discussed in Appendix I.

Neither the (sd) calculation nor the ZBM calculation is able to fit the 6.73 $\mathrm{MeV} \mathrm{O}^{+}$data, as shown in Figures $\mathrm{V}-45$ and $\mathrm{V}-46$. The curves are arbitrarily normalized to the data, giving isoscalar effective charges, $e_{0}$, of 2.6 and 2.5 for the $(\mathrm{sd})^{4}$ and ZBM calculations respectively. The ZBM calculation is unable to fit the 5.79-MeV angular distribution, shown in Figure V-47, but two-step routes have been shown to be very important in understanding that angular distribution. The second $1^{-}$in the ZBM calculation, when normalized to the data, fits the 8.71-MeV angular distribution, but it requires a large effective charge, 14 , and the second $1^{-}$ is not expected to correspond to the $8.71-\mathrm{MeV}$ peak. The $8.85-\mathrm{MeV}^{-}$, which the second ZBM state probably corresponds to, was not seen.

Because these calculations include the spin-flip part of the $\pi$-nucleus interaction, they can calculate transitions to unnatural-parity states such as the $2^{-}$shown in Figure V-48. The ZBM calculation fits the $180-\mathrm{MeV}$ data fairly well with an enhancement factor of 22 , although the calculation puts the maximum of the angular distribution about $10^{\circ}$ further out than the data. The $120-\mathrm{MeV} \pi^{+}$calculation bears no resemblance to the data. The cross section can bu approximated by

$$
\frac{\mathrm{d} \sigma(\theta)}{\mathrm{d} \Omega}=f(E)\left(4 \mathrm{M}^{2}(q) \cos ^{2}(\theta)+\sigma \cdot \hat{\mathrm{S}} \mathrm{S}^{2}(q) \sin ^{2}(\theta)\right)
$$

where the second term contains all the spin-Hip contributions (SI-81). If there are no two-step contributions to the $4.97-\mathrm{MeV}$ angular distributions, then only the second term can populate this state. For constant $q$ and $\theta<90^{\circ}$ the $180-\mathrm{MeV}$ cross sections will be smaller than the $120-\mathrm{MeV}$ cross sections. Also, the shapes of the 
TABLE V-6.

Effective charges extracted for ZBM and (sd) ${ }^{4}$ model spaces.

l Model Space

$e_{0}{ }^{1)}$

$2(\mathrm{sd})^{4}$

2.0

2 ZBM

2.2

$3 \quad 2 B M$

2.4

$4(s d)^{4}$

$2.2^{2)}$

4 ZBM

$\approx 6^{21}$

${ }^{1)} e_{0}$ is the isoscalar effective charge.

${ }^{2)}$ If two-step contributions to the $4.25-\mathrm{MeV}$ cross section are taken into account $e_{0}$ could be up to $40 \%$ larger. 


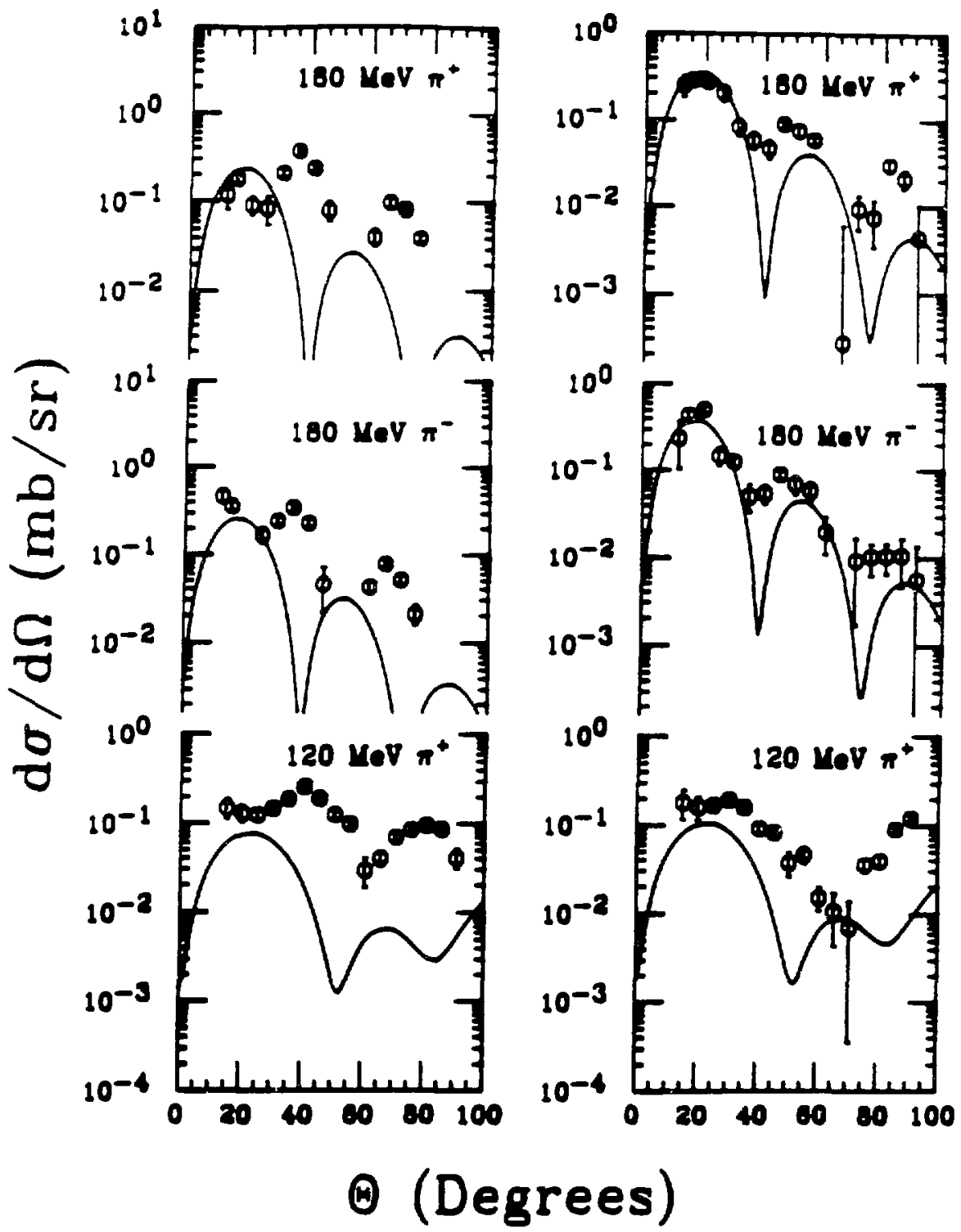

Figure V-47.

Angular diatributions for the 5.79-MeV and the 8.71-MeV 1- states. The top graph in each column is $180-\mathrm{MeV} \pi^{+}$, the middle $180-\mathrm{MeV}^{-}$, and the bottom $120-\mathrm{MeV} \pi^{+}$. The curven are DWLA calculations using microncopic transition densities from the ZBM shell-model calculation. 


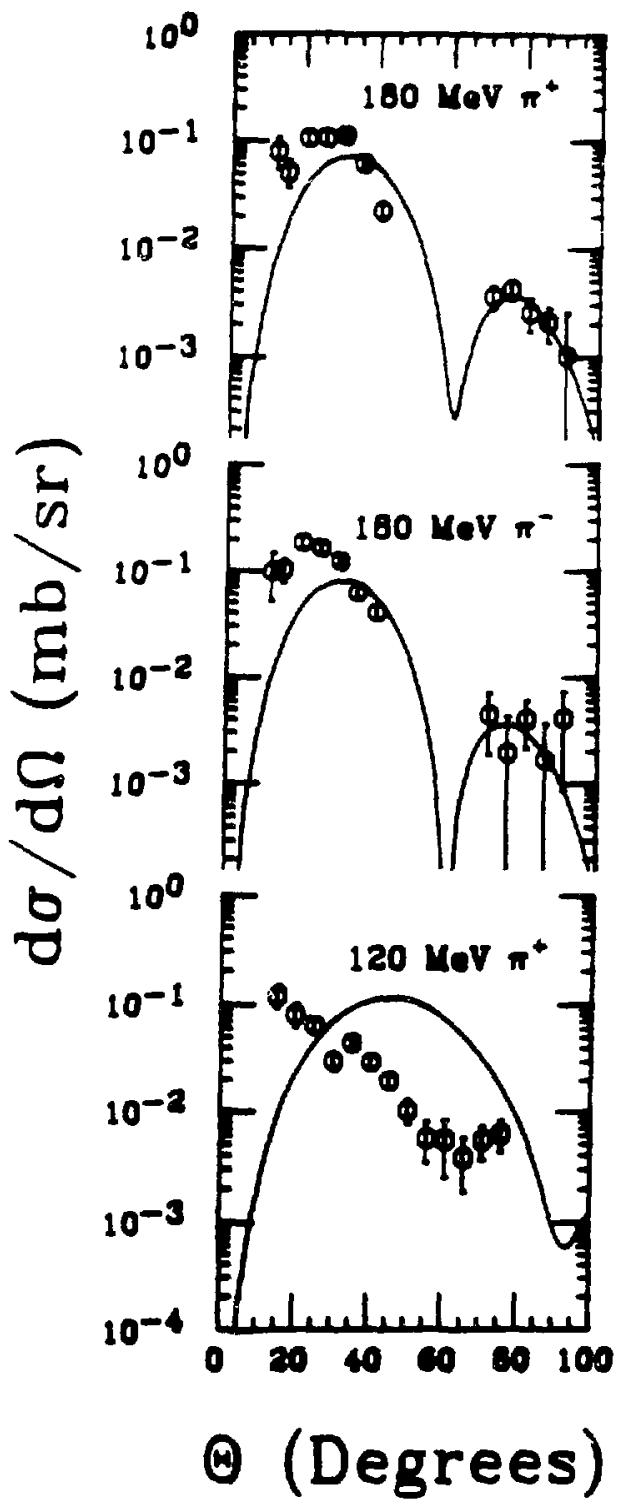

Figure V-48.

Angular distributions for the 4.97-MeV 2- state. The top graph is $180-\mathrm{MeV}$ $\pi^{+}$, the middle $180-\mathrm{MeV} \pi^{-}$, and the bottom $120 \mathrm{MeV} \pi^{+}$. The curvea are DWLA calculations using microscopic transition densities from the ZBM shellmodel calculation. 


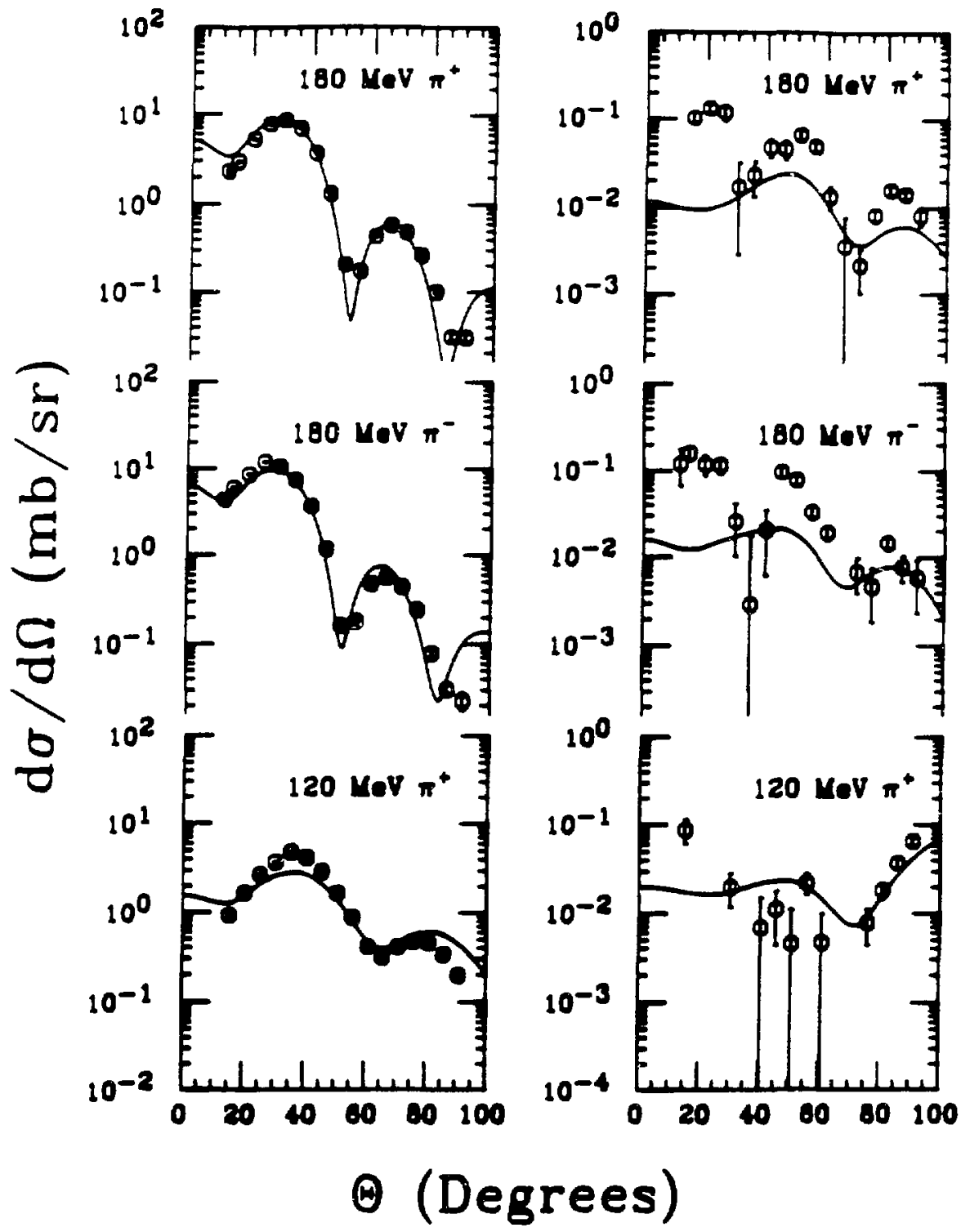

Figure V-49.

Angular distributions for the $1.63-\mathrm{MeV}$ and the $7.42-\mathrm{MeV} 2^{+}$states. The top graph in each column is $180-\mathrm{MeV} \pi^{+}$, the middle $180 \mathrm{MeV} \pi^{-}$, and the bot tom $120-\mathrm{MeV} \pi^{+}$. The curves are DWLA calculations using microscopic transition densities from the (sd) ${ }^{4}$ shell-model calculation. 


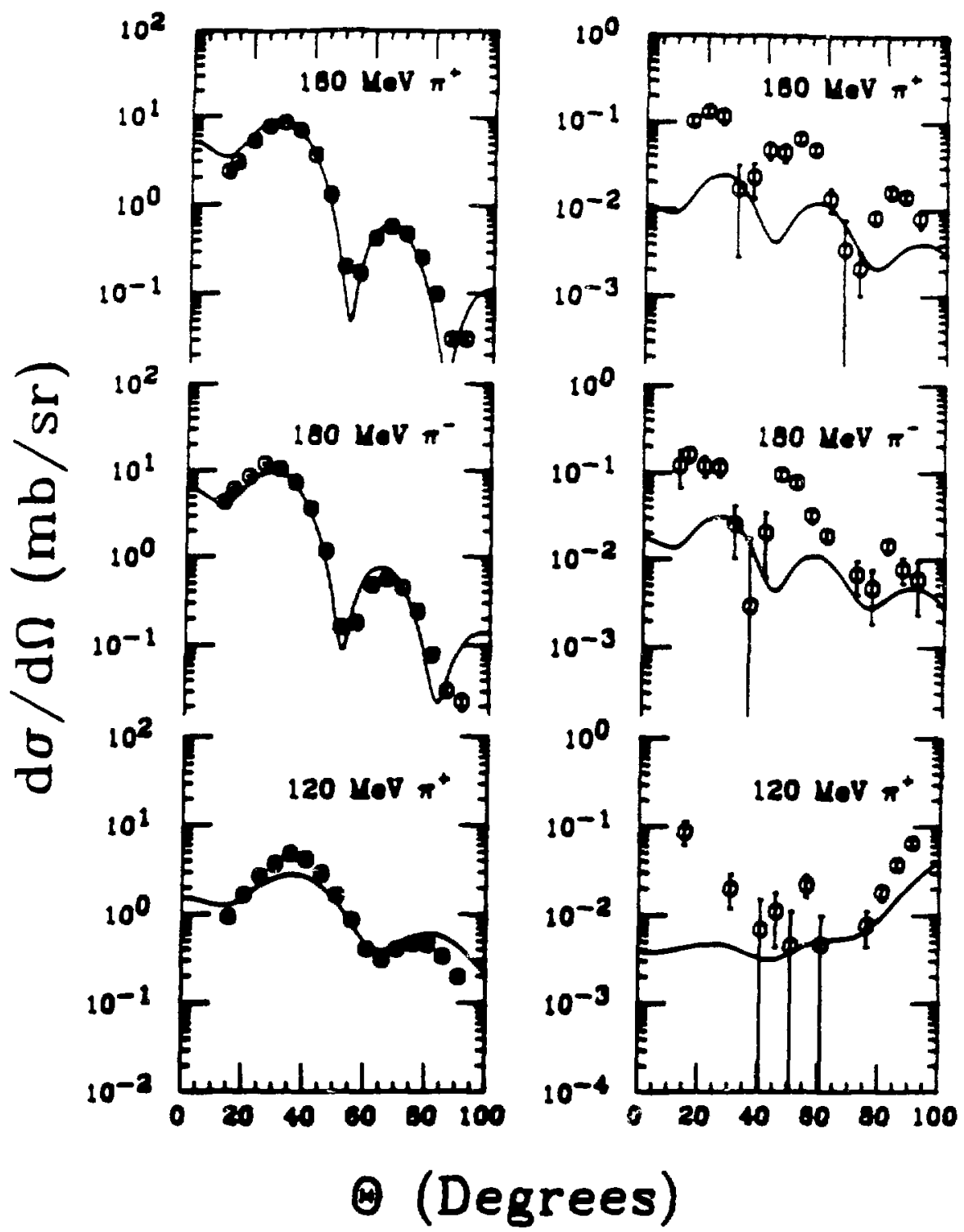

Ficure V-50.

Angular diatributions for the $1.63-\mathrm{MeV}$ and the $7.42-\mathrm{MeV}^{+}$states. The top graph in each column is $180 \mathrm{MeV} \pi^{+}$, the middle $180-\mathrm{MeV}^{-}$, and the bot tom $120-\mathrm{MeV} \pi^{+}$. The curre are DWIA calculations using microscopic transition densities from the 2BM shell-model calculation. 


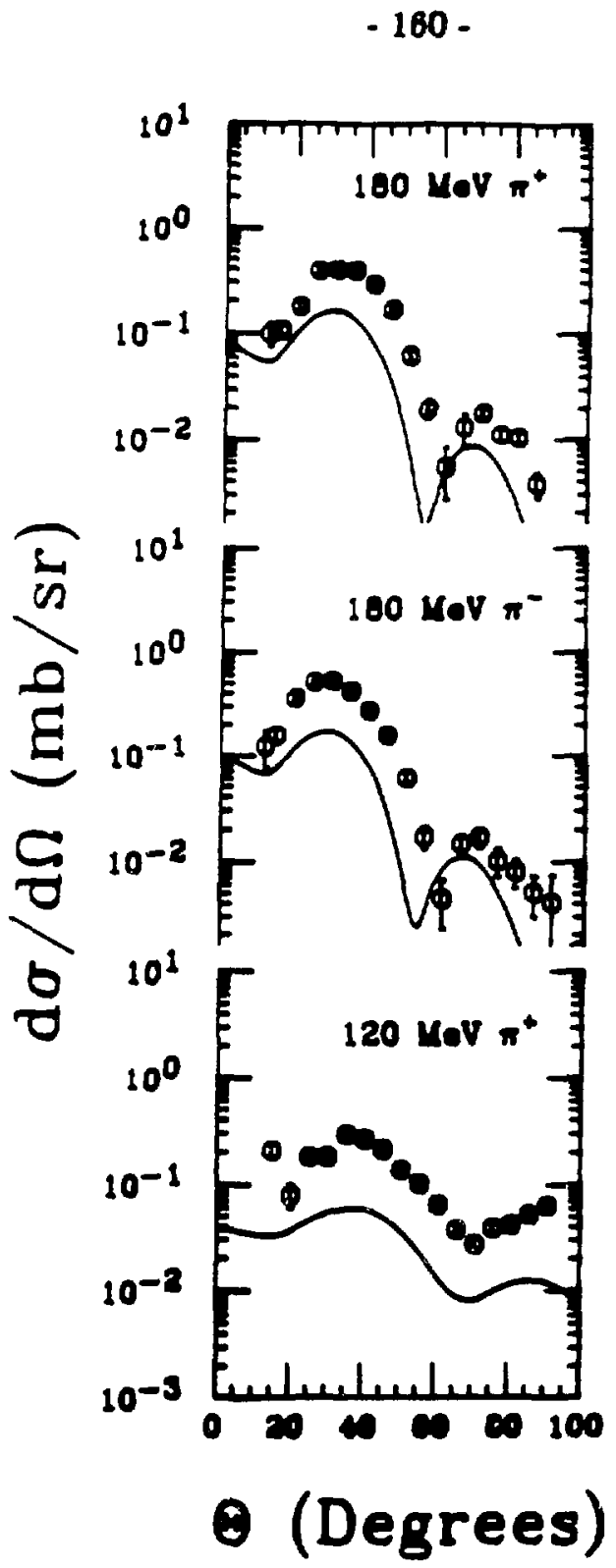

Figure V-51.

Same as Figure V-50 but for the 7.83-MeV state. 
angular distributions should be the same, with the maxima and minima at larger angles for the $120-\mathrm{MeV}$ data. The solid curves shown in Figure $\mathrm{V}-48$ have the correct behavior. The data clearly do not. This implies that two-step contributions are very important for this state.

The $1.63-\mathrm{MeV} 2^{+}$data, shown in Figures $\mathrm{V}-49$ and $\mathrm{V}-50$, were used to extract the isoscalar effective charge for $E 2$ transitions, $e_{0}=2.0$ for (sd) $)^{4}$ and $e_{0}=2.2$ for ZBM. Using these effective charges for the other $2^{+}$states, the ZBM calculation slightly underpredicts the 7.83-MeV angular distribution, shown in Figure V-51. Both calculations imply that the microscopic transition density is very important in understanding the $7.42 \mathrm{MeV}$ transition, shown in Figures V-49 and V-50.

Using $e_{0}=2.4$ the $2 B M$ calculation fits the $5.62-\mathrm{MeV} 3^{-}$very well, as shown in Figure V-52. If the second $3^{-}$in the calculation is assumed to correspond to the 7.16-MeV state the calculation underpredicts the cross section by a factor of 50 . However, the $7.16-\mathrm{MeV}$ state is believed to be formed by exciting one nucleon into the $\mathrm{fp}$ shell. The $10.41-\mathrm{MeV} 3^{-}$is more likely to correspond to the second $3^{-}$in the ZBM calculation. Figure V-53 shows that the calculation fits that state very well.

The (sd) calculations fit the $4.25-\mathrm{MeV}^{+}$with an effective charge of 2.2 , as shown in Figure V-54. The CCLA calculations showed the two-step route was also important in populating this state, so the value may be up to $40 \%$ larger. As seen in Figure V-54, using $e_{0}=2.2$ the (sd) ${ }^{4}$ calculation underpredicts the $9.99-\mathrm{MeV}^{+}$ by about an order of magnitude, but two-step contributions were also important for this state. The CCIA calculations required a much larger direct contribution, making the underprediction worse. 


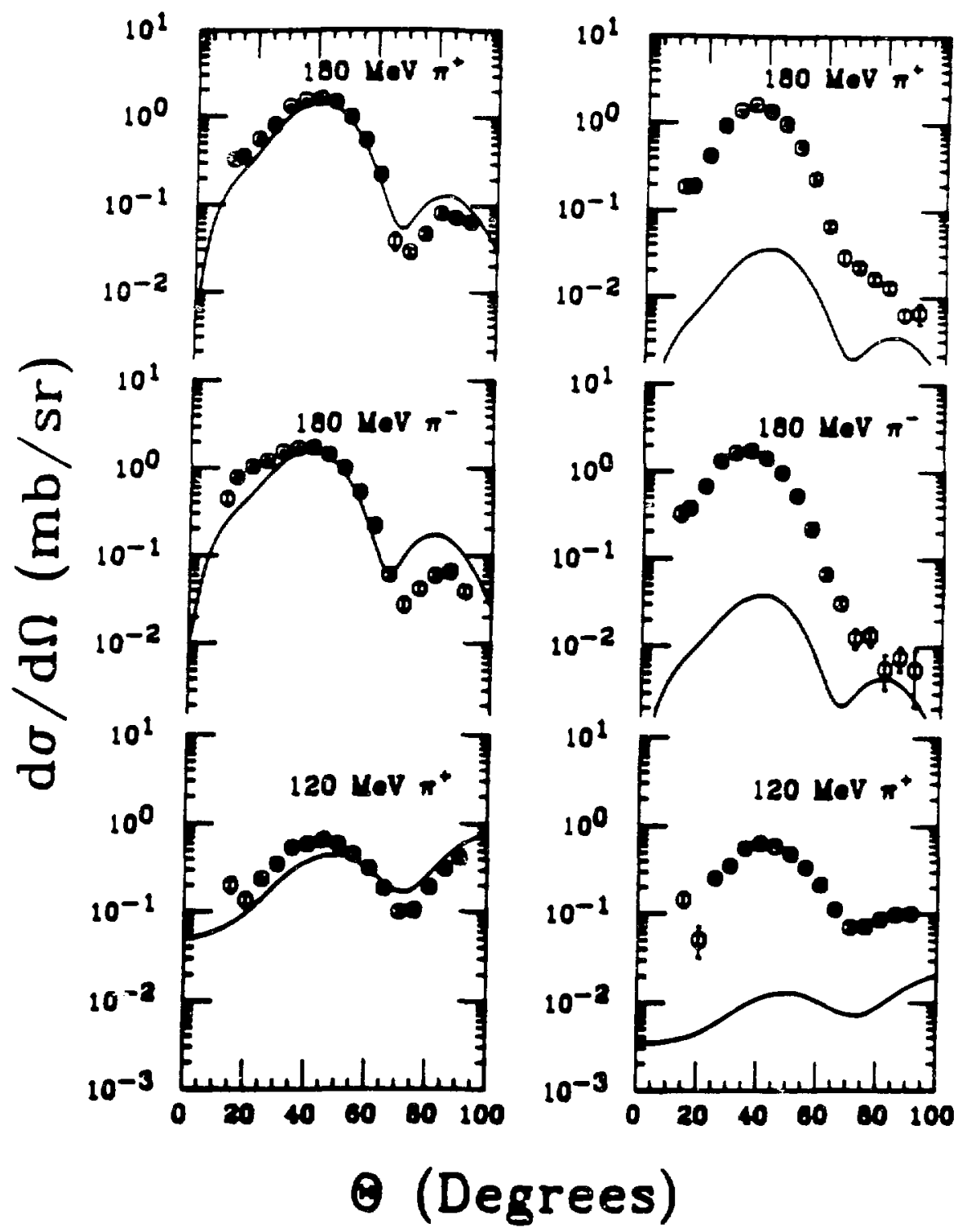

Figure V-52.

Angular distribution for the $5.62-\mathrm{MeV}$ and the $7.16-\mathrm{MeV} 3^{-}$staten. The top graph in each columo is $180 \mathrm{MeV} \pi^{+}$, the middle $180-\mathrm{MeV}^{-}$, and the bottom $120-\mathrm{MeV} \pi^{+}$. The curves are DWLA calculations using microscopic transition densities from the ZBM shell-model calculation. 


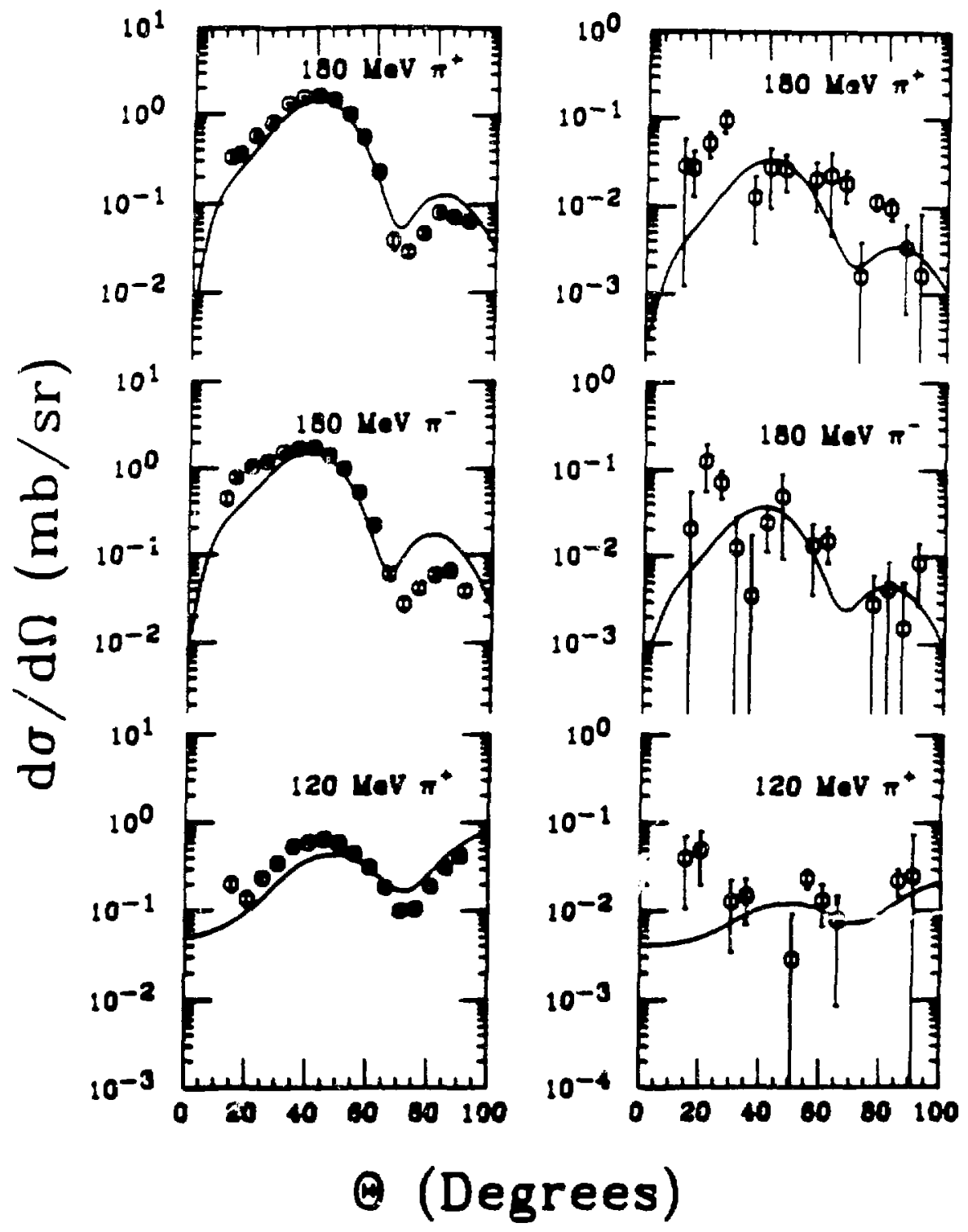

Figure V-53.

Same as Figure V.52 but for the $5.62-\mathrm{MeV}$ and $10.41-\mathrm{MeV}$ states. 


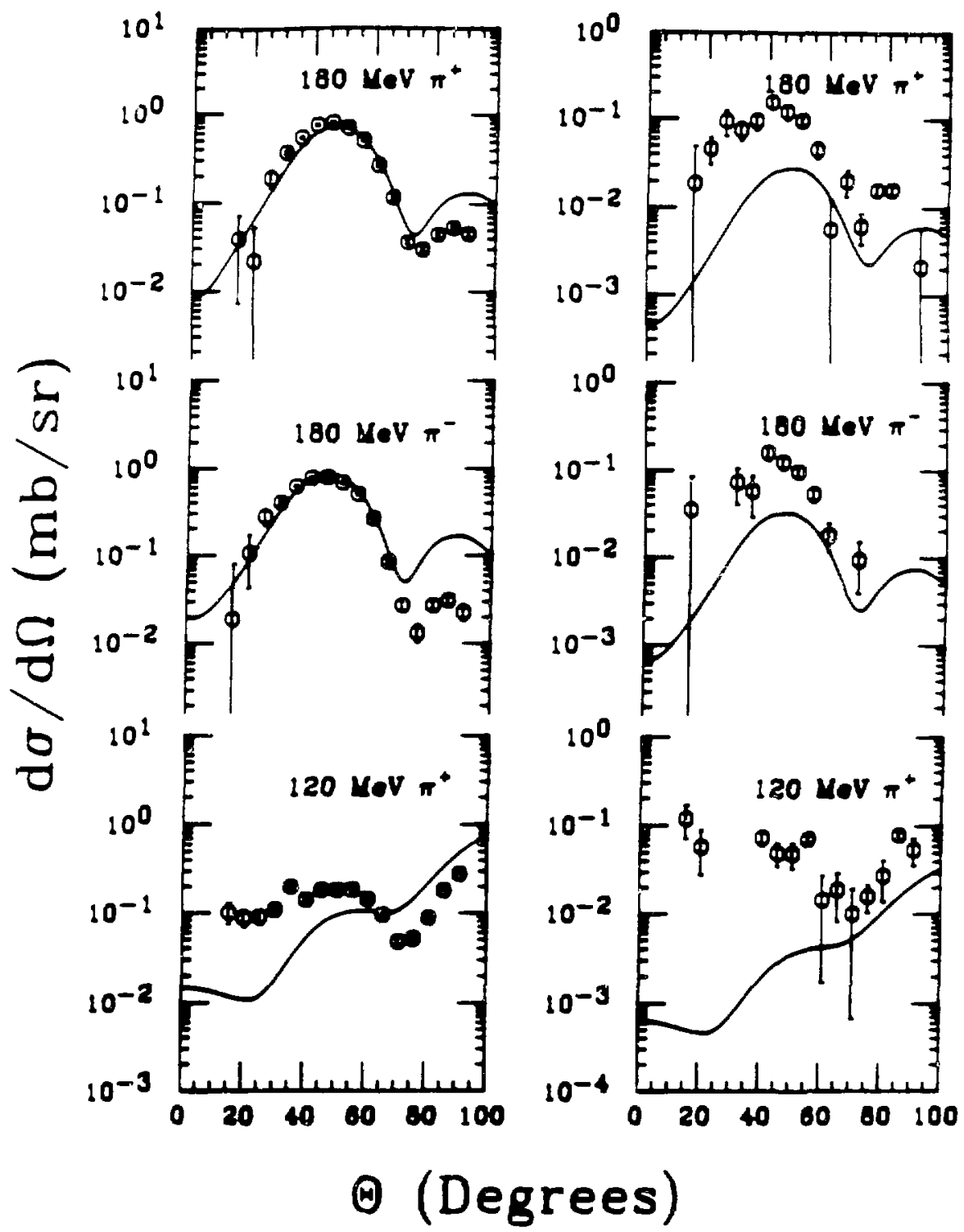

Ficure V-54.

Angular diatributions for the $4.25-\mathrm{MeV}$ and the $9.90-\mathrm{MeV}^{+}$states. The top graph in each column is $180-\mathrm{MeV} \pi^{+}$, the middle $180-\mathrm{MeV}^{-}$, and the bo:tom $120-\mathrm{MeV} \pi^{+}$. The curves are DWIA calculations using microscopic transition densities from the (sd) shell-model calculation. 


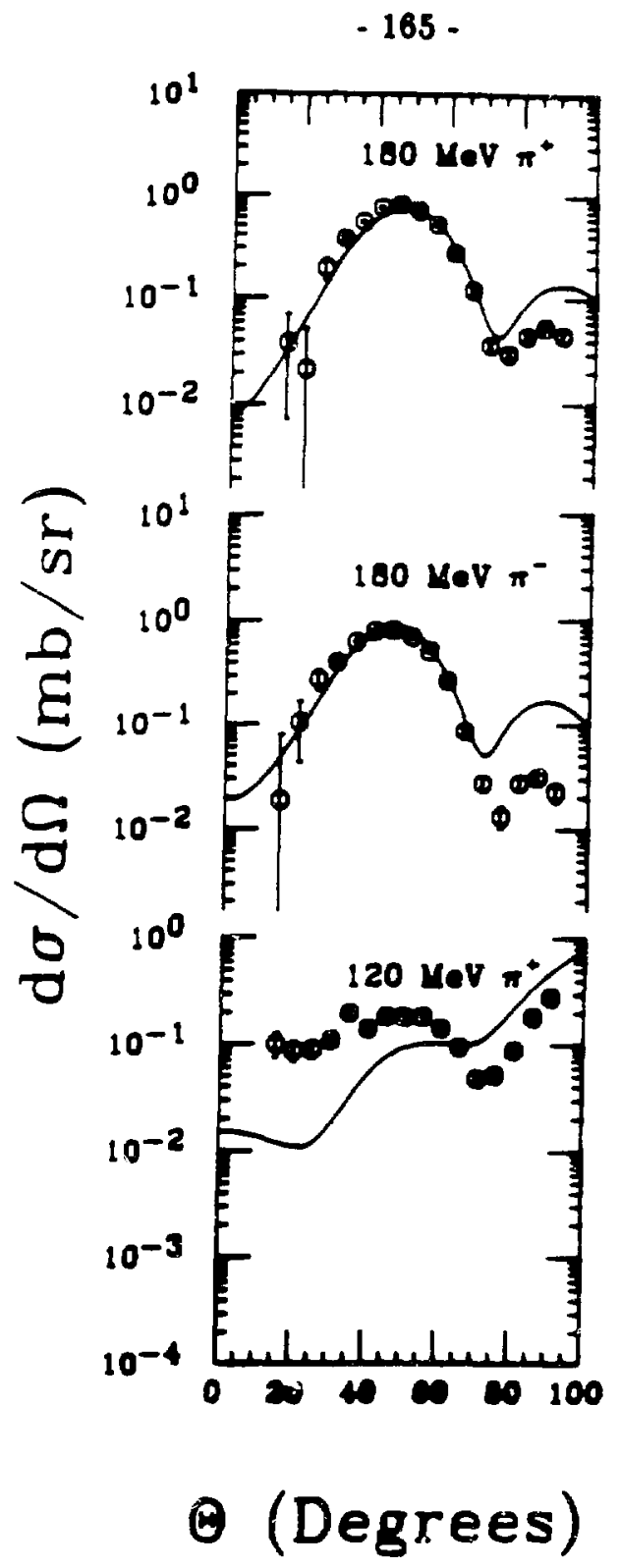

Figure $\checkmark-55$.

Angular distributions for the 4.25-MeV $4^{+}$state. The top graph is 180-MeV $\pi^{+}$, the middle $180-\mathrm{MeV} \pi^{-}$, and the bottom $120-\mathrm{MeV} \pi^{+}$. The curres are DWLA calculation using microscopis transition densitien from the ZBM shellmodel calculation. 


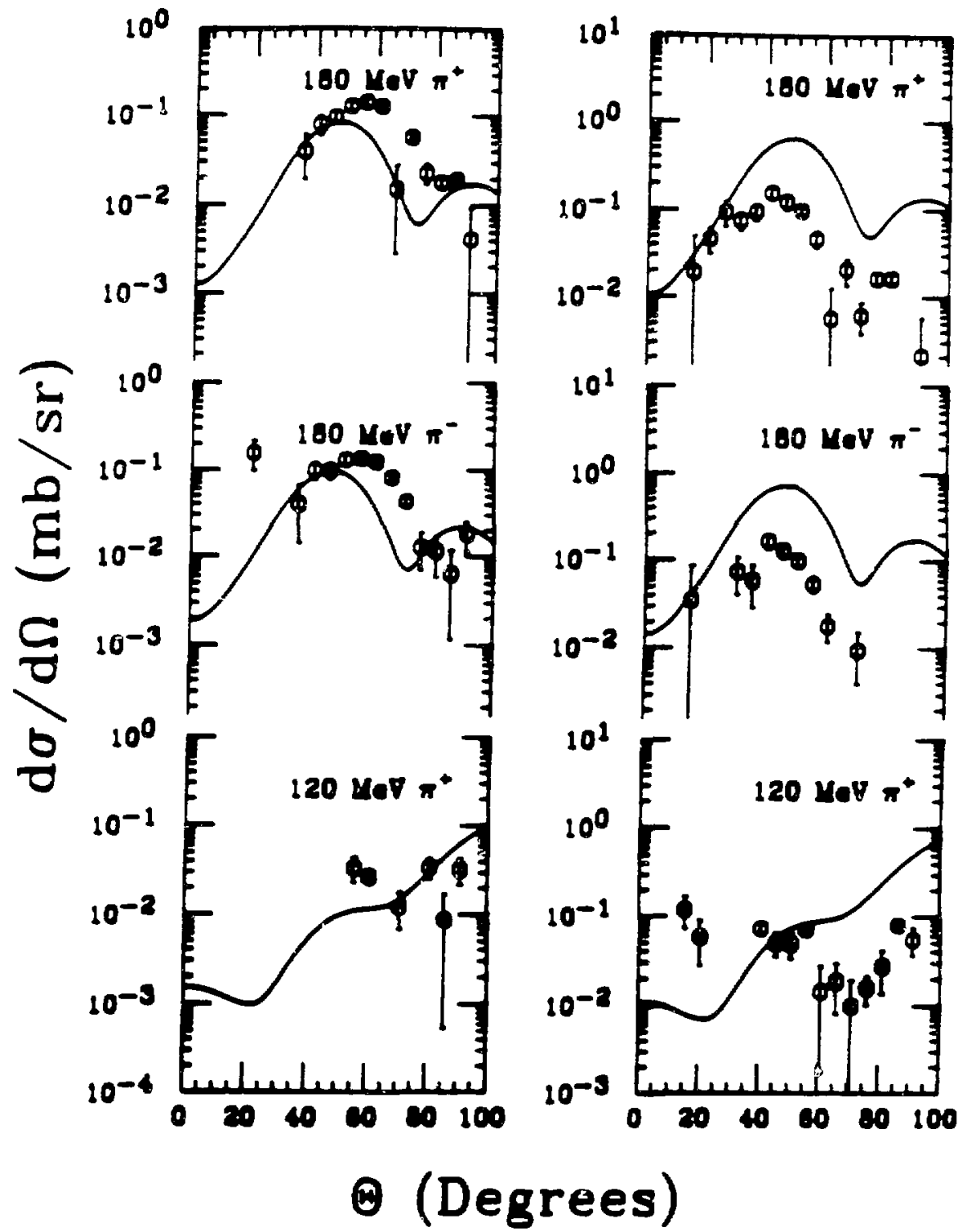

Figure V-se:

Angular dintribution for the $9.03-\mathrm{MeV}$ and $9.09-\mathrm{MeV} 4^{+}$states. The top graph in each column in $180-\mathrm{MeV} \pi^{+}$, the middle $180-\mathrm{MeV}^{-}$, and the bottom $120-\mathrm{MeV} \pi^{+}$. The currew are DWIA calculations using microscopic transition densitien from the ZBM shell-model calculation. 


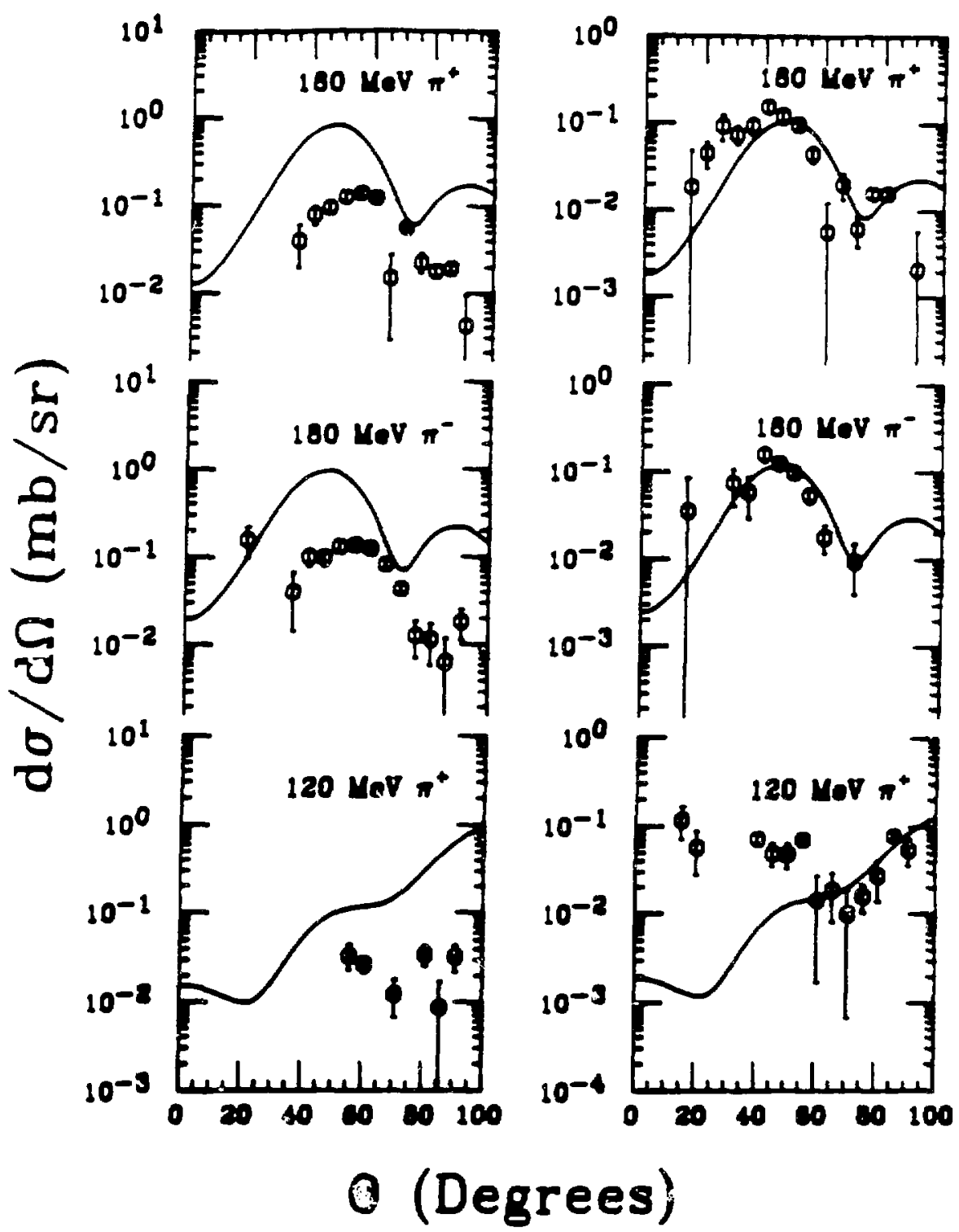

Figure V.57.

Same a Firure V-56 except asuming the $4^{+'}$ 's in the ZBM shell-model calculation switch as they do experimentally 
In the ZBM space the only way to construct an $l=4$ transition is with $\mathrm{d} 5 / 2-\mathrm{d} 5 / 2$, which may be why an effective charge of $\approx 6$ is necesgary to fit the 4.25-. $\mathrm{MeV}^{+}$, shown in Figure V-55. If the two-step contributions to the $4.25-\mathrm{MeV}$ angular distribution are included then a larger $e_{0}$ is again necessary. The 2 was some confusion in the $\mathrm{ZBM}$ calculation as to whether the second model state corresponded to the second or third physical state, and similarly for the third model state. Figures $V-56$ and $V-57$ show the results of each choice. Ignoring two-step contributions the third model state is able to fit either the second or the third $4^{+}$angular distribution, but the second model state overpredicts both by a factor of 5-10. However, the CCLA calculations showed construstive interference for the $9.03-\mathrm{MeV}_{4}^{-}$and destructive interference for the $9.99-\mathrm{MeV} 4$ so assuming the second model state goes with the third $4^{+}$and the third model state goes with the second $4^{-}$. . i.e. assuming the bands cross in the ZBM space like they do in the physical space, shows beiter agreement to the dats, although both calculations are probably still too large. 


\section{Conclusions}

This work is a study of pion inelastic scattering on ${ }^{20} \mathrm{Ne}$. Fifty-seven peaks are present. Most of the extracted angular distributions can be explained using the distorted-wave impulse approximation (DWIA) and a collective transition density. For some peaks DWLA was inadequate, or gave results that contradicted what was previously known about those states. Coupled-channels impulse approximation (CCLA) calculations were done to explain the transitions to many of these peaks.

The DWIA calculations fit all of the strong transitions geen. In general the extracted transition strengths agree with those measured by either $\gamma$ decay or inelastic electron scattering, given in Table VI-1 and shown in Figure 17-1. The only major disagreernent with $\gamma$ decay data is for a very weak transition to the 7.42 $\mathrm{MeV}$ state, where a number three times the $y$ decay upper limit was extracted. Because this state has a very antomalous angular distribution and was poorly fitted by both DWIA and CCLA calculations, the matrix element extracted here is clearly unreliable. This peak will be discussed later in this chapter. The transitions from the $4.25-\mathrm{MeV}$ gtate and the $7.16-\mathrm{MeV}$ state to the ground state have not been measured in $\gamma$ decay, but the excitations have been observed in inelastic electron 


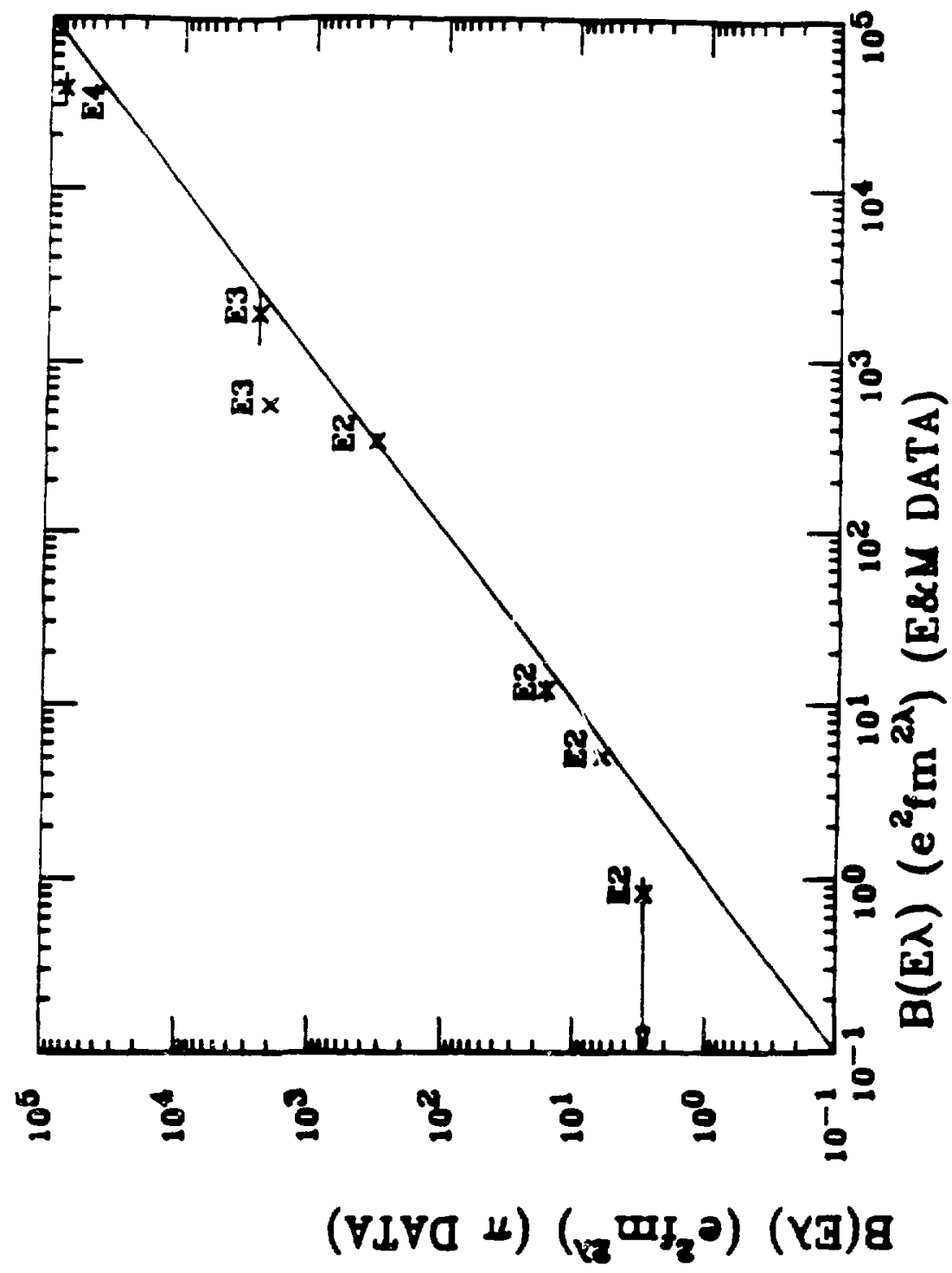

Figure VI-1.

Compariwa of $B(E)$ extracted with pion ecatteriog and electromarnetic probes. 
TABLE VI-1.

Comparison of $B(E$ ' $)$ 's from pion scattering with other data.

$\mathrm{E}_{\mathrm{x}} \quad \mathrm{J}^{\pi}$

$1.632^{+}$

$4.254^{+}$

$5.623^{-}$

$7.16 \quad 3^{-}$

$7.422^{+}$

$7.832^{+}$

$9.492^{+}$

$9.994^{+}$
Current exp.

323.

77300 .

2560.

2090.

2.85

16.6

6.09

16400 .
Compilation ${ }^{1)}$

$335 \pm 21$

$38000 \pm 8000^{21}$

$1883 \pm 632$

$\approx 550^{31}$

$\leq 0.82 \pm 0.17$

$12.0 \pm 1.5$

$\leq 5$

$\leq 5.6 \times 10^{\circ}$

1)Data from AJ-87.

${ }^{2)}$ Data from SI-73.

${ }^{3)}$ Data from MI-72.

TABLE VI-2.

Comparison of $B(E / y)$ 's from pion scattering with theory.

$\begin{array}{lllll}E_{\mathrm{x}} & J^{\pi} \quad \text { Current exp. } & Z^{\pi} \mathrm{BM}^{1)} & \text { (sd) }\end{array}$

$\begin{array}{lllll}1.62 & 2^{+} & 323 . & 233 & 303\end{array}$

$\begin{array}{lllll}4.25 & 4^{+} & 77300 . & 2873 . & 27600\end{array}$

$5.62 \quad 3^{-} \quad 2560$

1149.

$\begin{array}{lll}7.42 & 2^{+} & 2.85\end{array}$

0.255

0.163

$7.832^{+}$

16.6

4.52

$9.03 \mathrm{4}^{+}$

1830.

461

1160

$9.994^{+}$

16400.

3537.

$10.413^{-}$

41.3

28.4

1) $e_{0}=1$

${ }^{2)} e_{0}=1.7$ for E2 transitions and 2.0 for E4 transitions (BR-83). 
scattering (SI-73,MI-72). Our result for the $4.25-\mathrm{MeV}^{+}$is a factor of two higher than their result, and for the $7.16-\mathrm{MeV}^{-}$a factor of four higher. In both electron-scattering experiments the $7.16-\mathrm{MeV}^{-}$is not resolved from the $7.20-\mathrm{MeV}$ $0^{+}$. Unlike the current experiment, the $7.20-\mathrm{MeV}$ state dominates the peak in electron scattering. Their value for the transition strength was gotten by assuming the 7.16- $\mathrm{MeV}$ form factor had the same shape as the $5.62-\mathrm{MeV} 3_{1}^{-}$form factor and constraining the curve to 6 it the minimum of the measured form factor.

For many weaker transitions two-step routes were shown to be important. The $4^{+'}$ at 8.03 and $8.89 \mathrm{MeV}$ were poorly fitted by DWLA. Their angular distributions peaked at slightly larger and smaller angles than the DWLA calculation, respectively. DWLA calculations with an $l$ transfer of 3 fit the $9.99-\mathrm{MeV}$ dats almost as well as $/=4$. The first $4^{+}$at $4.25 \mathrm{MeV}$ was very well fitted with DWLA, but when the two-step route was taken into account the exiracted matrix element, $\mathrm{M}_{\mathrm{p}, \mathrm{a}}$, increased by almost $50 \%$. Because ${ }^{20} \mathrm{Ne}$ is an sd-shell nucleus, there should not be any direct $l=6$ excitations and any direct $l=5$ excitations must involve putting a nucleon in the fp-shell. The one $6^{+}$observed can be explained entirely with two-step excitations, although the quality of the data is so poor that a direct component can not be ruled out. Two peaks observed could correspond to known 5states in ${ }^{20} \mathrm{Ne}$. The peak at $10.26 \mathrm{MeV}$ is fitted by a one-step $l=5$ calculation. The two-step route makes no improvement in the fit and is virtually negligible. However, the states in this band are believed to result from exciting one nuclean into the fp-shell, so this is not a problem. The $8.45-\mathrm{MeV}$ peak is fitted very well with an $!=2$ DWLA calculation. Using known trangition strengths the two-step caiculation significantly overpredicts the large-angle data. A large direct E5 component was necessary to destructively interfere with the two-step route to bring the CCLA calculation down to the strength of the data. 
Because the standard collective transition density does not give a zero matrix element for $l=0$ and $1(\Delta T=0)$ transitions, different forms were used (AU-71,HA81). For the $6.73-\mathrm{MeV} \mathrm{O}^{+}$, a two-step route, with the $1.63-\mathrm{MeV}^{+}$as the intermediate state, is non-negligible. The transition density of Auerbach $\left(A C^{-71}\right)$ does not fit the data. A phenomenological form for the transition density used in electron scattering, (MI-72,SI-73), fitted the angular distribution and gave a transition strength of $5.27 \mathrm{fm}^{2}$, in good agreement with the electron scattering results of $7.37 \pm 1.97 \mathrm{fm}^{2}(\mathrm{MI}-72)$ or $5.85 \pm 1.5 \mathrm{fm}^{2}$ (SI-73). The results for the $l=1$ transition density are not as clear. The $5.79-\mathrm{MeV}$ peak is fitted very well using two two-step routes and a direct route in the CCLA calculation. The other low-lying $1^{-}$observed is the $8.71-\mathrm{MeV}$ state, which can be fitted either with DWLA using the transition density of Harakeh and Dieperink (HA-81) or with a two-step route using the 1.63- $\mathrm{MeV} 2^{+}$as the intermediate state, but any CCLA calculation using both routes fit the data worse than either route separately. Since the strengths of each route could not be determined from other data or calculations it as not possible to constrain either route. Other possible $1^{-}$states observed are at higher excitation and are fitted as well with the standarf collective transitions density as with the density of Harakeh and Dieperink (HA-81).

The data to low-lying states bave also been fitted using microscopic transition densities from shell-model calculations, described in Chapter 3. The transition strengths extracted with collective iransition densities are rompared to the predicted strengths in Table VI: 2 and Figure VI-2. For the (sd) ${ }^{4}$ calculation the agreement is very good. For the ZBM calculation the agreement is fairly good. although there are some significant discrepancies. The caiculation does not seem to get the mixing between the $2^{+}$and $4^{+}$states of the second and third rotational bands correct. This was also apparent in Chapter 3 where the calculation was 

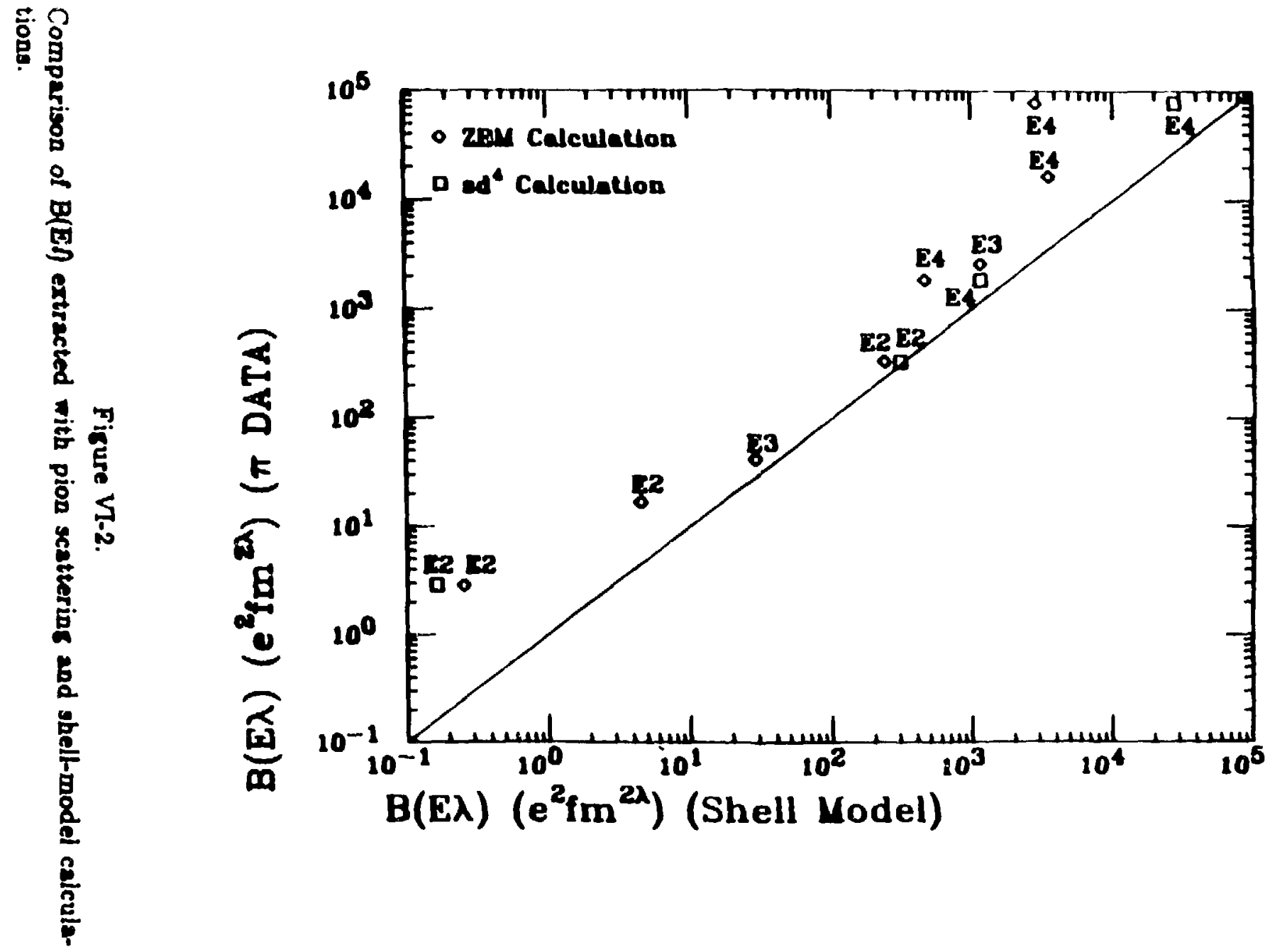

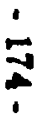


compared to $\gamma$ decay data. Using microscopic transition densities from these calculations many of the extracted angular distributions were fitted. Most of the discrepancies arise for states where a two-step route was also seen to be important. The peculiar angular distribution of the $7.42-\mathrm{MeV}^{+}$appears to arise from degtructive interference between the $1 d 5 / 2-1 d 5 / 2,1 d 5 / 2-2 s 1 / 2$, and $2 s 1 / 2-1 d 5 / 2$ amplitudes in the shell-model calculations.

For strong transitions, DWLA using a collective transition density fits the data very well and gives results that agree with other probes. While DWLA does sometimes work for weaker transitions, this is not always the case. Two-step routes, even when they are much weaker than the direct route, can have a dramatic effect on the extracted transition matrix element, $M_{p, a}$. Stronger two-step routes can change the shape of the angular distribution, moving maxima and minima enough that one might deduce an incorrect $/$ transfer. For the $8.71-\mathrm{MeV}^{-}$the data were unable to distinguish between the direct and two-step routes. When one or more twostep routes dominate, very anomalous and mislesding angular distributions can result. Also important for weaker peaks are microscopic effects. Cancellations between shell-model amplitudes can give very anomalous angular distributions which in regions where the density of states is high might lead one to incorrectly deduce the peak is a doublet. 


\section{Appendix 1. High Excitation Peaks}

Above $8 \mathrm{MeV}$ there are states in ${ }^{20} \mathrm{Ne}$ which are not a part of any rotational band. They will be discussed in this section, except for peaks which require $l=1$ transitions to explain the angular distributions. There are several $\mathrm{K}^{\pi}=0^{+}$bands in ${ }^{20} \mathrm{Ne}$ not discussed in Chapter 5 given in the compilation (AJ-87). They are generally at much higher excitation and unambiguous identifcation of their states is not possible. No peaks observed in the current experiment appear to correspond to states in those bands. There are no other negative-parity bands identified in ${ }^{20} \mathrm{Ne}$. States needing some $1=1$ component to explain their angular distributions have been discussed in Chapter 5.

Figures A1-1 through A1-21 show angular distributions and DWLA fits for 24 peaks between $8.71 \mathrm{MeV}$ and $17.13 \mathrm{MeV}$ in excitation. All of the fits were done requiring $M=M_{p}=M_{n}$, and varying $M$ to fit the $180-\mathrm{MeV}^{+}$and $\pi^{-}$data. The extracted values of $M$ are given in Table A1-1. In many cases, particulariy where large $l$ transfers are indicated, the choice of $l$ transfer is ambiguous. All $l s$ which give reasonable fits have been shown. The 1 transfer extracted with DWLA may have little relation to the actual $J^{\pi}$ of the state because of two-step contributions, 
and because the transition density may have a very non-collective shape due to microscopic effects.

Nine peaks are fitted with $l$ transfers greater than 5 . Because $l=4$ is the largest $/$ transfer possible in the sd shell, $l=5$ if one particle is allowed to excite into the fp-shell, these states probably have significant two-step contributions to their cross sections. The peak at $12.03 \mathrm{MeV}$ might correspond to the $6^{+}$member of the $\mathrm{K}^{\pi}=\mathrm{O}_{3}{ }^{+}$band at $12.14 \mathrm{MeV}(\mathrm{AJ}-87)$. The $6^{+}$member of the $\mathrm{K}^{\pi}=\mathrm{O}_{2}{ }^{+}$band has been postulated to be either the state at $12.585 \mathrm{MeV}$ or $13.105 \mathrm{MeV}$ (AJ-8i) and may correspond to the peak at 12.58 or $13.08 \mathrm{MeV}$.

Figures A1-22 through A1-36 show 14 peaks which could not be fitted reasonably with one $l$ transfer assuming $M_{n}=M_{p}$. Nine of these peaks can only be fitied with the incoherent sum of $2 r s$, implying that these peaks are doublets. The extracted transition strengths are included in Table A1-1.

The first $\mathrm{T}=1$ state in ${ }^{20} \mathrm{Ne}$ occurs at $10.27 \mathrm{MeV}$. There are 12 known $\mathrm{T}=1$ states in ${ }^{20} \mathrm{Ne}, 3$ possible $\mathrm{T}=1$ states, and 22 states with no isospin information in the compilation (AJ-87). Shell-model calculations of the structure of these states have been done using both model spaces described in Chapter 3. The isovector effective charge is believed to be one in the sd shell (BR-82,AL-85). The isovector effective charge, $e_{1}$, is the difference between the neutron and proton effective charges, $e_{n}$ and $e_{p}$. Writing the neutron and proton effective charges as

$$
e_{p}=\left(1+\delta_{p}\right) e \text { and } e_{n}=\left(0+\delta_{v}\right) e,
$$

it is obvious that the statement $e_{1}=1$ is equivalent to the statement that $\delta_{p}=\delta_{D}$, i.e. the change in charge is the same for neutrons and protons. Several peaks seen above $10 \mathrm{MeV}$ could correspond to $\mathrm{T}=1$ states in ${ }^{20} \mathrm{Ne}$. They are listed in Table A1-2. Assuming the $T=1$ state is the only state contributing significantly to the 
peak, then the isovector effective charge can be extracted using the computer codes PIPIT (EI-76b) and HL (LE-80b) to calculate the angular distributions. For the ZBM calculation it was assumed that the first shell-model state of a given $\mathrm{J}^{\pi}$ corresponded to the first experimental state of the same $\mathrm{J}^{\pi}$, the second to the second and so on. The states in ${ }^{20} \mathrm{~F}$ with predominantly (sd) ${ }^{4}$ structure are known, (CR-74), and their analogs in ${ }^{20} \mathrm{Ne}$ are also known, (AJ-87). The extracted isovector effective charges are given in Table A1-2. If other states contribute to the cross section then the extracted effective charges are upper limits.

Pion inelastic scattering is very sensitive to differences between the neutron and proton parts of the transition density. It can be used to find strong neutron or proton excitations. Looking at difference spectra, i.e. a spectrum formed by subtracting a normalized $\pi^{-}$spectrum from a $\pi^{+}$spectrum, has found siveral of these states (SE-81). If a state has good isospin it should not appear in the difference spectrum, but if a state is primarily a neutron or proton excitation it will show up as a peak or dip in the difference spectrum. There is no evidence in these spectra for any states which are primarily neutron or proton excitations in ${ }^{20} \mathrm{Ne}$. One would not expect to see any differences below the energy of the first $T=1$ state at 10.27 MeV, because a state which is a neutron or proton excitation would have mixed isospin - while the nuclear force will produce only states of good isospin, the Coulomb force, generally relatively weak in light nuclei, will mix states of good isospin. It is probably not surprising that nothing is seen in the difference spectra, because above $10 \mathrm{MeV}$ the density of states is high and there are no strongly excited peaks. 
TABLE A1-1.

Ground-state transition strengths extracted with DWLA calculations ${ }^{1)}$,

\begin{tabular}{|c|c|c|c|}
\hline Energy & P! & $M\left(e\left(m^{\prime}\right)^{3)}\right.$ & $B(E / 1)\left(e^{2} \mathrm{fm}^{2 \eta}\right)$ \\
\hline 9.12 & 3 & $1.17(0.09) \times 10^{1}$ & $1.37 \times 10^{2}$ \\
\hline \multirow[t]{2}{*}{9.28} & 2 & $1.0(0.5) \times 10^{0}$ & $1.02 \times 10^{0}$ \\
\hline & 3 & $5.3(2.3) \times 10^{0}$ & $2.86 \times 10^{1}$ \\
\hline 9.45 & 2 & $2.47(0.20) \times 10^{0}$ & $6.09 \times 10^{0}$ \\
\hline \multirow[t]{2}{*}{9.88} & 3 & $6.2(1.3) \times 10^{0}$ & $3.86 \times 10^{1}$ \\
\hline & 4 & $3.3(0.9) \times 10^{1}$ & $1.08 \times 10^{3}$ \\
\hline \multirow[t]{5}{*}{10.63} & $2+4$ & $\left.1.6(0.4) \times 10^{0} 5\right)$ & $2.7 \times 10^{0}$ \\
\hline & & $<4.9(0.8) \times 10^{14)}$ & $<2.4 \times 10^{3}$ \\
\hline & 2 & $1.75(0.14) \times 10^{0}$ & $3.06 \times 10^{0}$ \\
\hline & 3 & $1.02(0.09) \times 10^{1}$ & $1.04 \times 10^{2}$ \\
\hline & 4 & $5.7(0.5) \times 10^{1}$ & $3.29 \times 10^{3}$ \\
\hline \multirow[t]{3}{*}{10.90} & 2 & $1.99(0.11) \times 10^{0}$ & $3.95 \times 10^{0}$ \\
\hline & 3 & $1.22(0.06) \times 10^{1}$ & $1.48 \times 10^{2}$ \\
\hline & 4 & $7.1(0.4) \times 10^{1}$ & $5.06 \times 10^{3}$ \\
\hline \multirow[t]{4}{*}{11.08} & $2+5$ & $\left.1.01(0.17) \times 10^{05}\right)$ & $1.02 \times 10^{0}$ \\
\hline & & $\left.2.0(0.4) \times 10^{26}\right)$ & $4.2 \times 10^{4}$ \\
\hline & 3 & $\left.7.2(1.1) \times 10^{05}\right)$ & $5.2 \times 10^{1}$ \\
\hline & 4 & $\left.<4.9(1.0) \times 10^{14}\right)$ & $<2.4 \times 10^{3}$ \\
\hline \multirow[t]{3}{*}{11.64} & 2 & $7.9(1.8) \times 10^{-1}$ & $6.27 \times 10^{-1}$ \\
\hline & 4 & $2.5(0.7) \times 10^{1}$ & $6.18 \times 10^{2}$ \\
\hline & 6 & $9.4(2.2) \times 10^{2}$ & $8.80 \times 10^{5}$ \\
\hline \multirow[t]{2}{*}{11.83} & 3 & $4.4(1.5) \times 10^{0.5)}$ & $1.90 \times 10^{1}$ \\
\hline & 4 & $2.3(0.8) \times 10^{(5)}$ & $5.22 \times 10^{2}$ \\
\hline \multirow[t]{3}{*}{12.03} & 6 & $1.93(0.17) \times 10^{3}$ & $3.72 \times 10^{0}$ \\
\hline & 7 & $1.38(0.11) \times 10^{4}$ & $1.81 \times 10^{8}$ \\
\hline & 8 & $1.14(0.08) \times 10^{5}$ & $1.30 \times 10^{10}$ \\
\hline \multirow[t]{4}{*}{12.22} & $2+4$ & $\left.2.0(0.2) \times 10^{0} 5\right)$ & $4.10 \times 10^{0}$ \\
\hline & & $\left.9.5(0.5) \times 10^{15}\right)$ & $9.31 \times 10^{3}$ \\
\hline & 3 & $\left.5.8(0.2) \times 10^{0} 6\right)$ & $3.3 \times 10^{1}$ \\
\hline & 4 & $\left.3.33(0.13) \times 10^{15}\right)$ & $1.11 \times 10^{3}$ \\
\hline \multirow[t]{3}{*}{12.38} & 6 & $1.3(0.2) \times 10^{3}$ & $1.67 \times 10^{8}$ \\
\hline & 7 & $9.5(1.7) \times 10^{3}$ & $9.07 \times 10^{7}$ \\
\hline & 8 & $8.2(1.5) \times 10^{4}$ & $6.64 \times 10^{9}$ \\
\hline
\end{tabular}


TABLE A1-1.

Ground-state transition strengths extracted with DWIA calculations ${ }^{1)}$.

\begin{tabular}{|c|c|c|c|}
\hline Energy & f) & $\left.M\left(e f m^{i}\right)^{3}\right)$ & $B(E l i)\left(e^{2} f m^{2 l}\right)$ \\
\hline \multirow[t]{2}{*}{12.58} & 6 & $1.08(0.20) \times 10^{3}$ & $1.17 \times 10^{6}$ \\
\hline & 9 & $8.0(1.0) \times 10^{5}$ & $6.36 \times 10^{11}$ \\
\hline 12.85 & 2 & $3.78(0.07) \times 10^{0}$ & $1.43 \times 10^{1}$ \\
\hline \multirow[t]{2}{*}{13.08} & 5 & $1.8(0.3) \times 10^{2}$ & $3.34 \times 10^{4}$ \\
\hline & 6 & $1.13(0.17) \times 10^{3}$ & $1.27 \times 10^{0}$ \\
\hline \multirow[t]{2}{*}{13.43} & 6 & $1.53(0.12) \times 10^{3}$ & $2.35 \times 10^{\circ}$ \\
\hline & 7 & $1.08(0.09) \times 10^{4}$ & $1.16 \times 10^{8}$ \\
\hline 13.57 & 2 & $1.60(0.16) \times 10^{0}$ & $2.55 \times 10^{0}$ \\
\hline 13.94 & 2 & $2.75(0.08) \times 10^{0}$ & $7.58 \times 10^{0}$ \\
\hline 14.14 & 2 & $1.42(0.14) \times 10^{0}$ & $2.02 \times 10^{0}$ \\
\hline \multirow[t]{3}{*}{14.34} & 5 & $1.5(0.4) \times 10^{2}$ & $2.16 \times 10^{4}$ \\
\hline & 6 & $9 .(3.) \times 10^{2}$ & $8.68 \times 10^{6}$ \\
\hline & 7 & $7 .(2.) \times 10^{3}$ & $4.33 \times 10^{7}$ \\
\hline \multirow[t]{2}{*}{14.44} & 5 & $1.8(0.5) \times 10^{2}$ & $3.19 \times 10^{4}$ \\
\hline & 6 & $10 .(3.) \times 10^{2}$ & $9.83 \times 10^{6}$ \\
\hline \multirow[t]{3}{*}{14.64} & $2+4$ & $\left.2.39(0.14) \times 10^{06}\right)$ & $5.73 \times 10^{0}$ \\
\hline & & $\left.4.3(0.6) \times 10^{15}\right)$ & $1.8 \times 10^{3}$ \\
\hline & 2 & $\left.2.59(0.13) \times 10^{05}\right)$ & $6.73 \times 10^{0}$ \\
\hline \multirow[t]{3}{*}{15.13} & $2+4$ & $2.3(0.4) \times 10^{0.5)}$ & $5.3 \times 10^{0}$ \\
\hline & & $<3.8(0.6) \times 10^{14)}$ & $1.5 \times 10^{3}$ \\
\hline & 2 & $2.4(0.4) \times 10^{0.5)}$ & $5.8 \times 10^{0}$ \\
\hline \multirow[t]{3}{*}{$: 5.36$} & $2+4$ & $2.08(0.19) \times 10^{05}$ & $4.33 \times 10^{0}$ \\
\hline & & $\left.3.9(0.6) \times 10^{16}\right)$ & $1.5 \times 10^{3}$ \\
\hline & 2 & $\left.2.29(0.14) \times 10^{05}\right)$ & $5.24 \times 10^{0}$ \\
\hline \multirow[t]{4}{*}{15.74} & $2+4$ & $\left.1.1(0.3) \times 10^{05}\right)$ & $1.13 \times 10^{0}$ \\
\hline & & $\left.1.9(1.1) \times 10^{15}\right)$ & $3.8 \times 10^{2}$ \\
\hline & 2 & $1.2(0.3) \times 10^{0.6)}$ & $1.33 \times 10^{0}$ \\
\hline & 3 & $\left.5.9(1.5) \times 10^{0} 5\right)$ & $3.5 \times 10^{1}$ \\
\hline \multirow[t]{2}{*}{15.91} & 2 & $1.57(0.13) \times 10^{0}$ & $2.46 \times 10^{0}$ \\
\hline & 3 & $7.9(0.7) \times 10^{0}$ & $6.21 \times 10^{1}$ \\
\hline 16.13 & 3 & $1.14(0.05) \times 10^{1}$ & $1.31 \times 10^{2}$ \\
\hline \multirow[t]{2}{*}{16.35} & $2+5$ & $\left.1.2(0.3) \times 10^{0.5}\right)$ & $1.5 \times 10^{0}$ \\
\hline & & $\left.2.1(0.4) \times 10^{25}\right)$ & $4.28 \times 10^{4}$ \\
\hline
\end{tabular}


TABLE A1-1.

Ground-state transition strengths extracted with DWLA calculations ${ }^{13}$.

$\begin{array}{llll}\text { Energy } & p^{2} & M(\mathrm{e} \mathrm{fm})^{3)} & B\left(E(1)\left(\mathrm{e}^{2} \mathrm{fm}^{2}\right)\right. \\ 16.57 & 2+4 & 1.5(0.2) \times 10^{06)} & 2.20 \times 10^{0} \\ & & 6.2(0.5) \times 10^{15} & 3.90 \times 10^{3} \\ & 2+5 & 1.8(0.2) \times 10^{05} & 3.1 \times 10^{0} \\ & & \left.3.5(0.3) \times 10^{25}\right) & 1.19 \times 10^{5} \\ 16.82 & 2 & 1.45(0.13) \times 10^{0} & 2.09 \times 10^{0} \\ 16.98 & 3 & 7.1(1.4) \times 10^{0} & 5.06 \times 10^{1} \\ 17.13 & 5 & 2.3(0.3) \times 10^{2} & 5.31 \times 10^{4}\end{array}$

1) Table V-2 includes states in the compilation, (AJ-87), which may correspond to the peaks listed here.

${ }^{2)} l$ is the angular momentum transfer used to fit the data. If more than one $l$ is listed then several $r_{s}$ were used to fit the data separately and the $M$ listed is for each fit. If $l_{1}+b_{2}$ is listed then $2 l_{s}$ were added incoherently to fit the data and the first $M$ listed is for $l_{1}$, the second $M$, on the next line, is for $l_{2}$.

${ }^{3)}$ Except where noted all M's were obtained by fitting $180 \mathrm{MeV}^{+}$and $\pi^{-}$data simultaneously, with the constraint $M=M_{n}=M_{p}$. Results for peaks with $l=0$ or 1 contributions are in Table V-5. The number in parentheses is the statistical error of the fit.

4) $\mathrm{M}$ was obtained by taking the maximum from either the $180 \mathrm{MeV}^{+}$or $\pi^{-}$data. The number in parentheses is the statistical error of the fit taken.

${ }^{6} \mathrm{M}$ was obtained by averaging the values for $180 \mathrm{MeV} \pi^{+}$and $\pi^{-}$. The number in parentheses is the statistical error of the fit. 
TABLE A1-2.

Possible isovector transitions in ${ }^{20} \mathrm{Ne}\left(\pi, \pi^{\prime}\right)$.

$\begin{array}{llllll}\text { Energy } & l & \mathrm{E}_{\mathrm{x}}{ }^{\prime \prime} & \mathrm{J}^{\pi} ; \mathrm{T}^{1)} & \mathrm{e}_{1}{ }^{2)} & \mathrm{e}_{1}{ }^{3)} \\ 10.90 & 3 & 10.88 & 3^{+} ; 1 & 4.5^{4)} & 3.9^{4)} \\ 11.08 & 4 & 11.09 & 4^{+} ; 1 & 2.9 & 3.8 \\ 11.22 & 1 & 11.26 & 1^{+} ; 1 & 6.7^{4)} & 3.2^{4)} \\ & & 11.27 & 1^{-} ; 1 & 1.3 & - \\ 11.64 & 2 & 11.60 & 2^{-} ; 1 & 2.1^{4)} & - \\ 12.22 & 2 & 12.22 & 2^{+} ; 1 & 2.9 & 4.7 \\ & 3 & 12.26 & 3^{-} ; 1 & 3.0 & - \\ 13.57 & 2 & 13.59 & 2^{+} & & \\ 13.72 & 1 & 13.74 & 1^{+} & & \\ 13.94 & 2 & 13.88 & 2^{+} ; 1 & 2.6 & 3.8 \\ 14.14 & 2 & 14.06 & 2^{+} & & \\ & & 14.13 & 2^{-} & & \\ 14.88 & 1 & 14.70 & \left(1^{+}\right) & & \\ & & 14.78 & \left(1^{-}\right) & & \end{array}$

1)Data from AJ-87.

${ }^{2)}$ Extracted for the ZBM model space.

${ }^{3)}$ Extracted for the (sd) ${ }^{4}$ model space.

4)For unnatural parity states the square root of $\mathrm{N}$ is listed, where

$$
\mathrm{N}=\frac{\sigma_{\exp }}{\sigma_{\text {theo }}}
$$




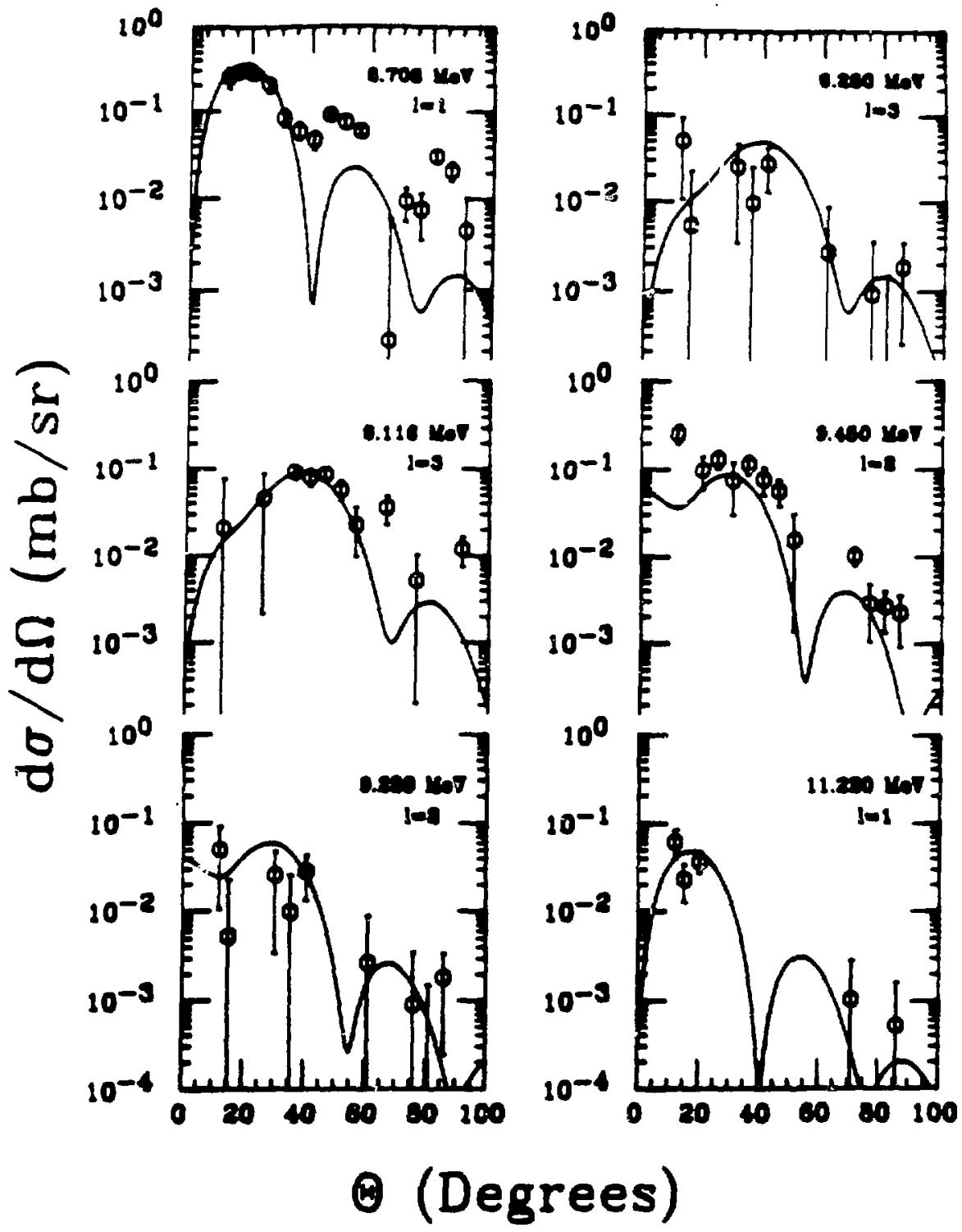

Figure Al-1.

Angular distributions for peake between 8.71 and $11.22 \mathrm{MeV}$ in excitation which could be it with a single l. All of the graphs in both columan are 180 $\mathrm{MeV} \pi^{+}$. The curves are DWLA calculations using a collective transition density. 


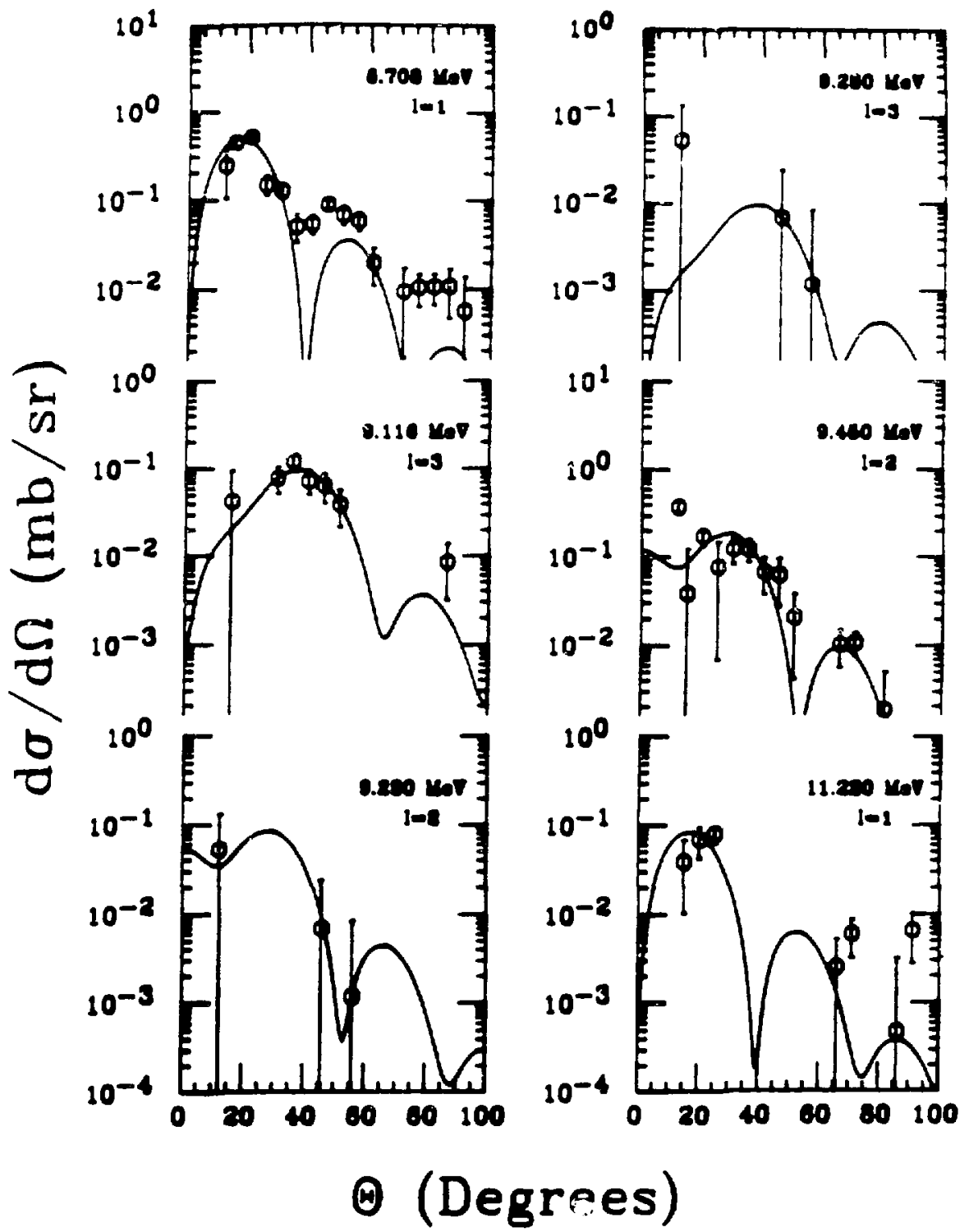

Figure A1-2.

Same as Figure A1-1 except the graphs are $180-\mathrm{MeV} \pi^{-3}$ data. 


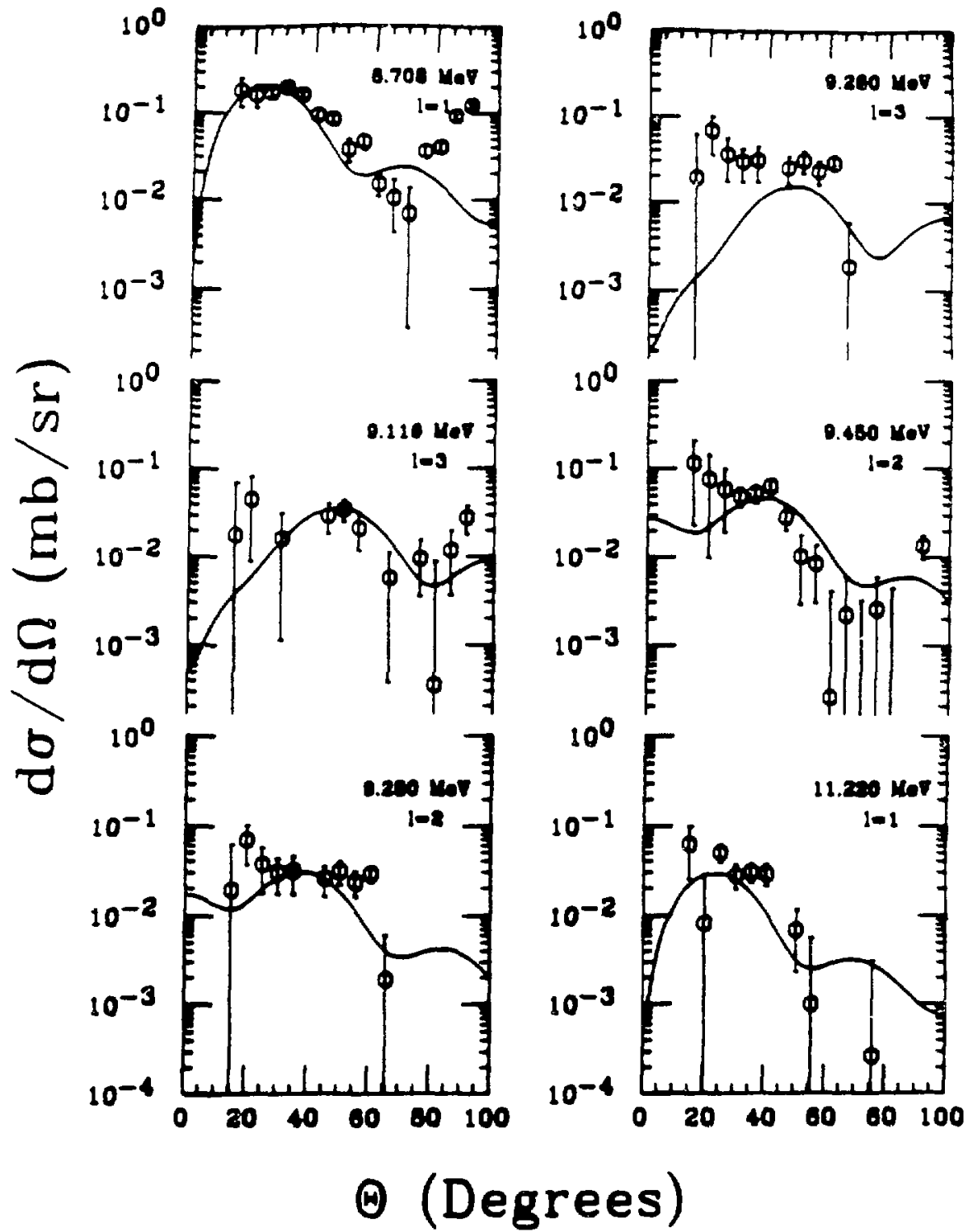

Figure A1-3.

Same as Figure A1-1 except the graphs are $120-\mathrm{MeV} \pi^{+}$data. 


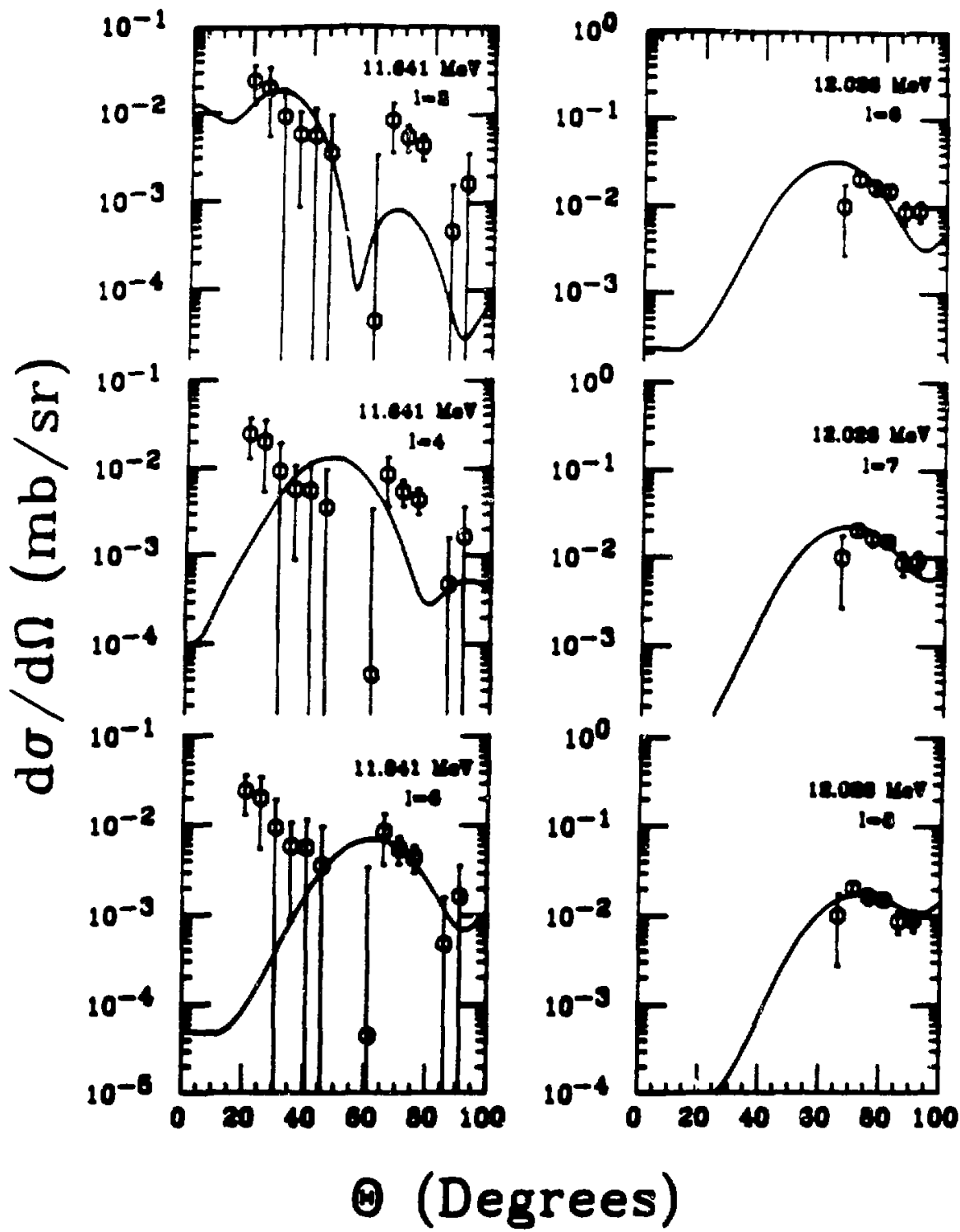

Ficure A1-4.

Same a Figure Al-1 but for stateo between 11.64 and $12.03 \mathrm{MeV}$ in excitetion. 


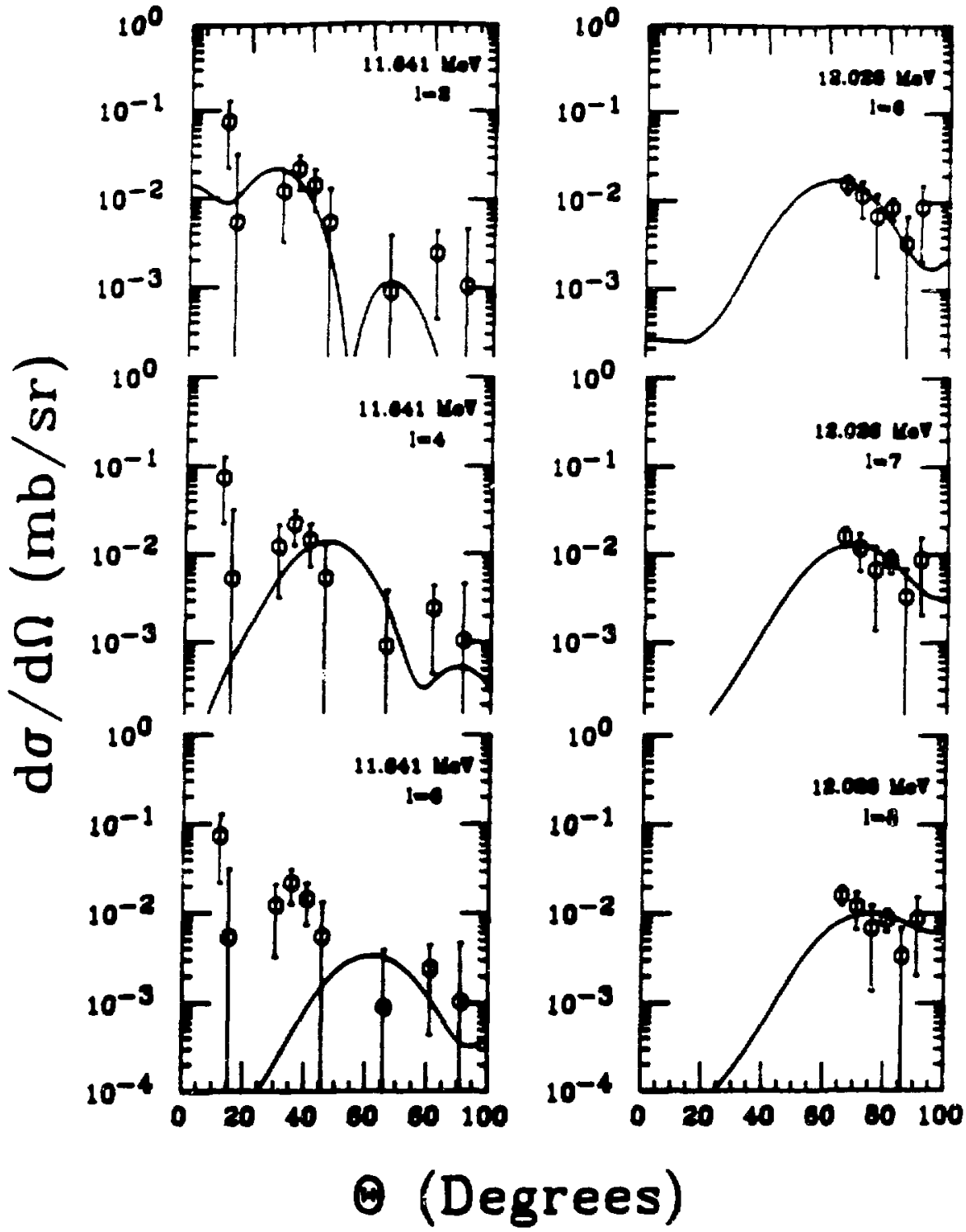

Figure A1-5.

Same a Figure A1-4 except the graphs are $180-\mathrm{MeV} \pi^{-}$data. 


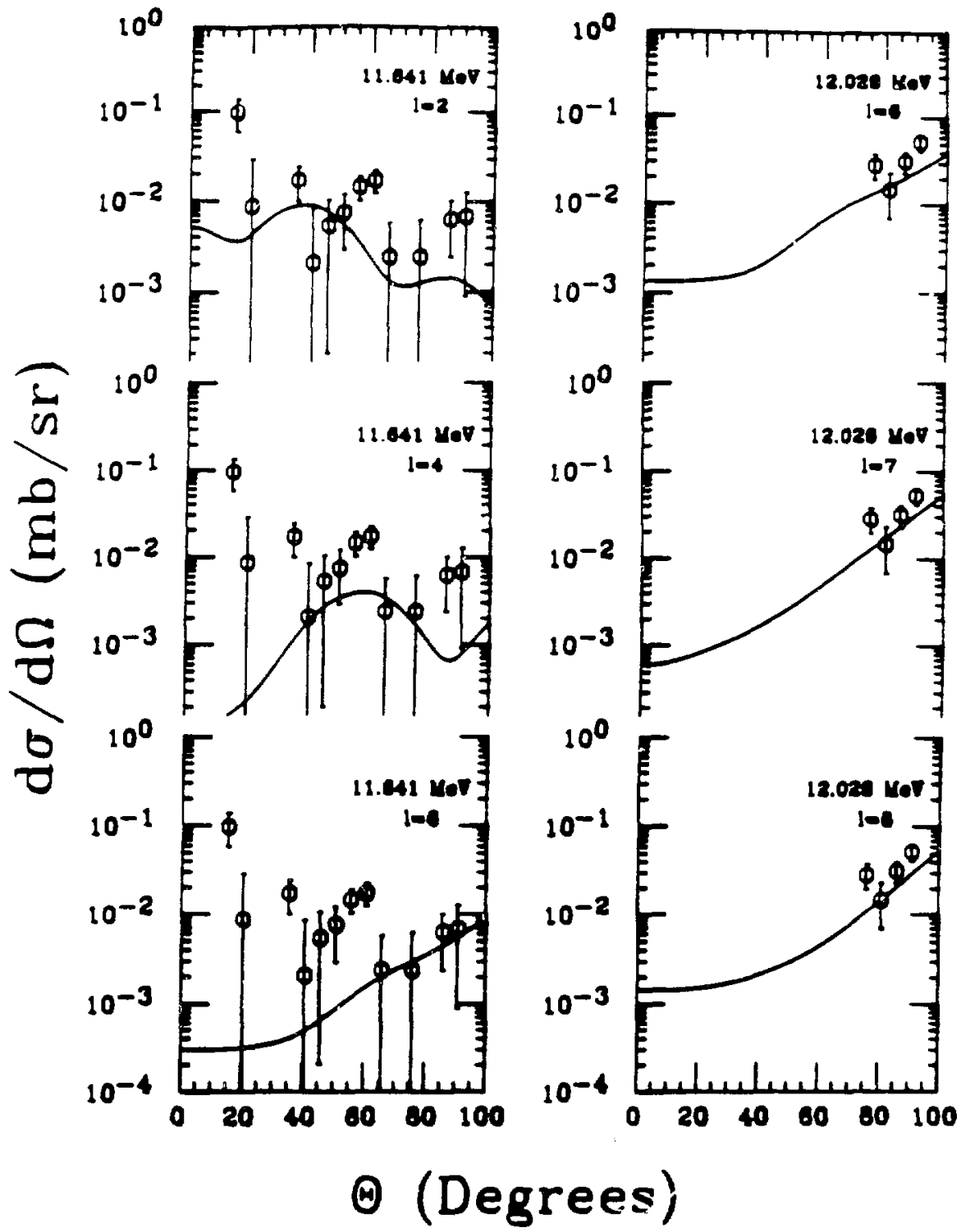

Figure A1-6.

Same as Figure A1-4 except the graphs are $120-\mathrm{MeV} \pi^{+}$dats. 


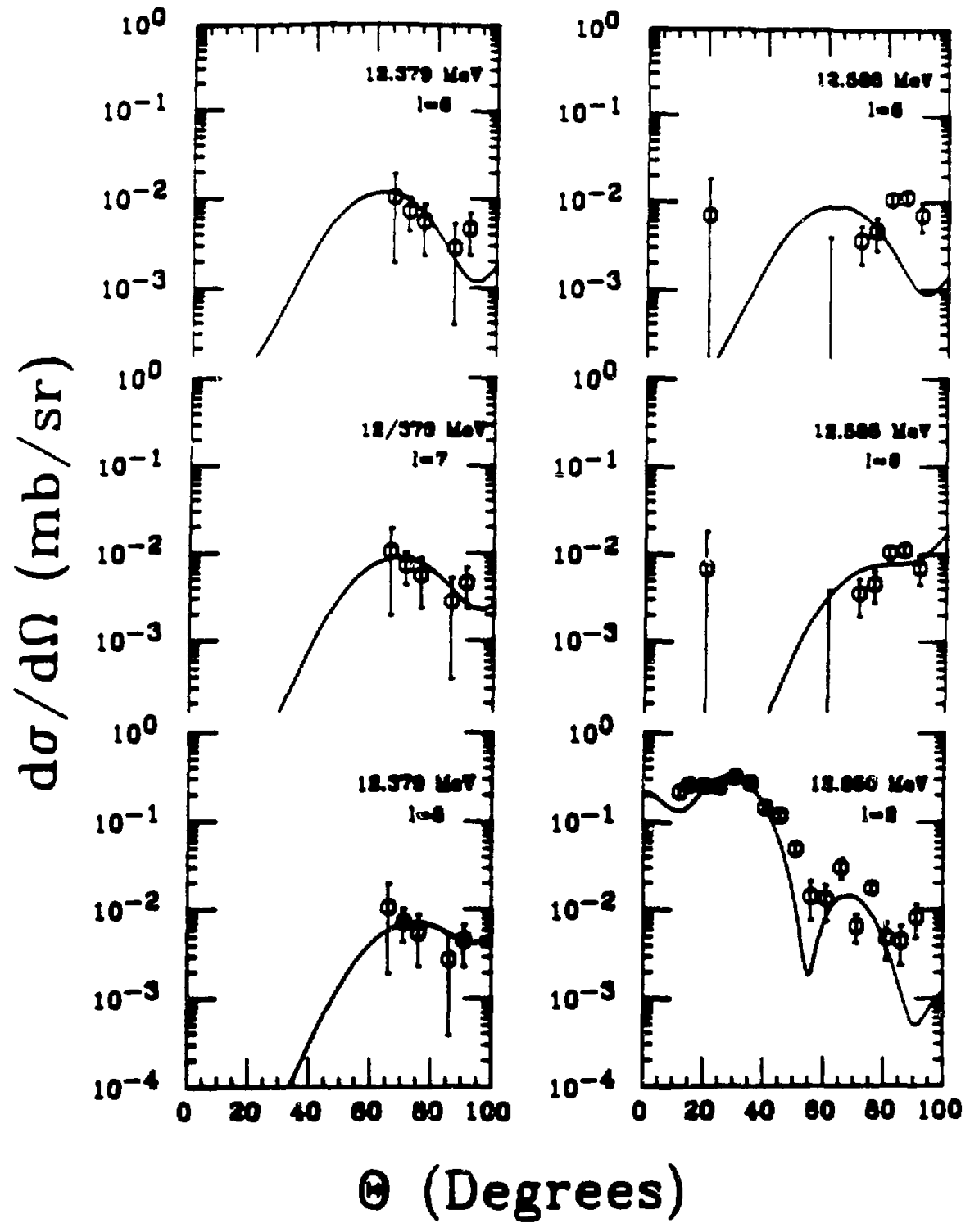

Firure A1-7.

Same as Figure Al-1 but for states between 12.38 and $12.85 \mathrm{MeV}$ in excitation. 


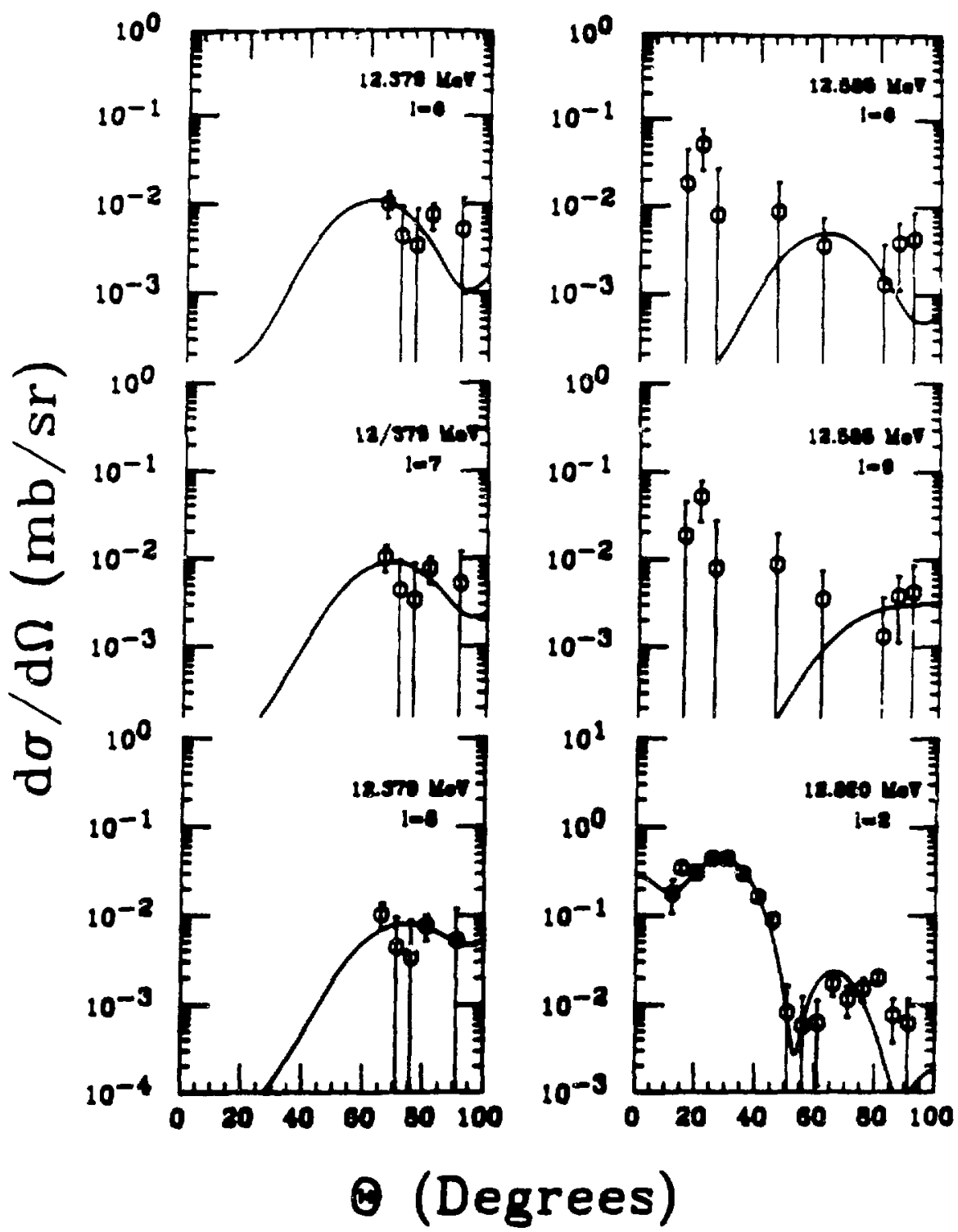

Figure A1-8.

Same as Figure Al-7 except toe graphs are $180-\mathrm{MeV} \pi^{-}$data. 


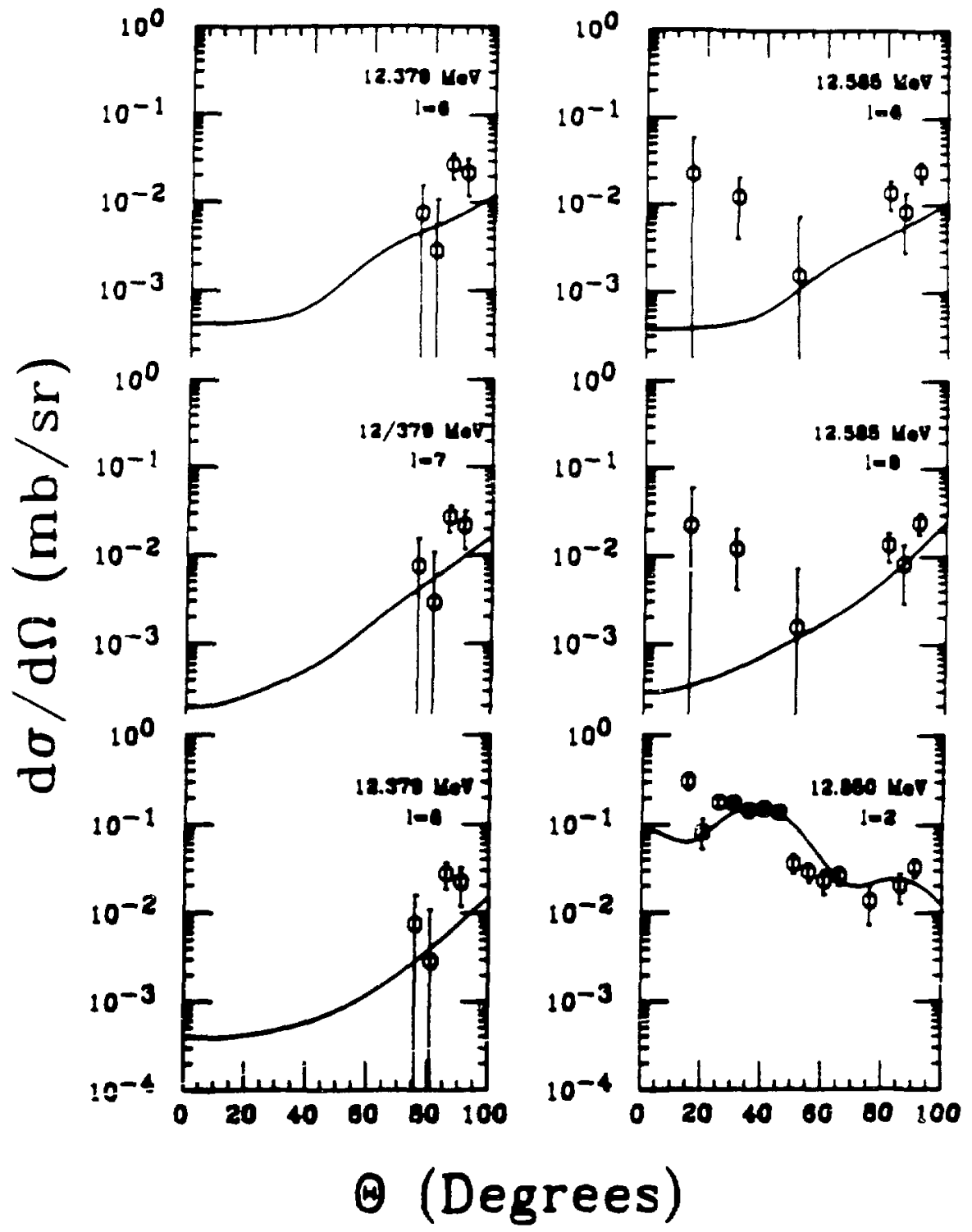

Figure A1-9.

Same as Figure A1-7 except the graphs are $120-\mathrm{MeV} \pi^{+}$data. 


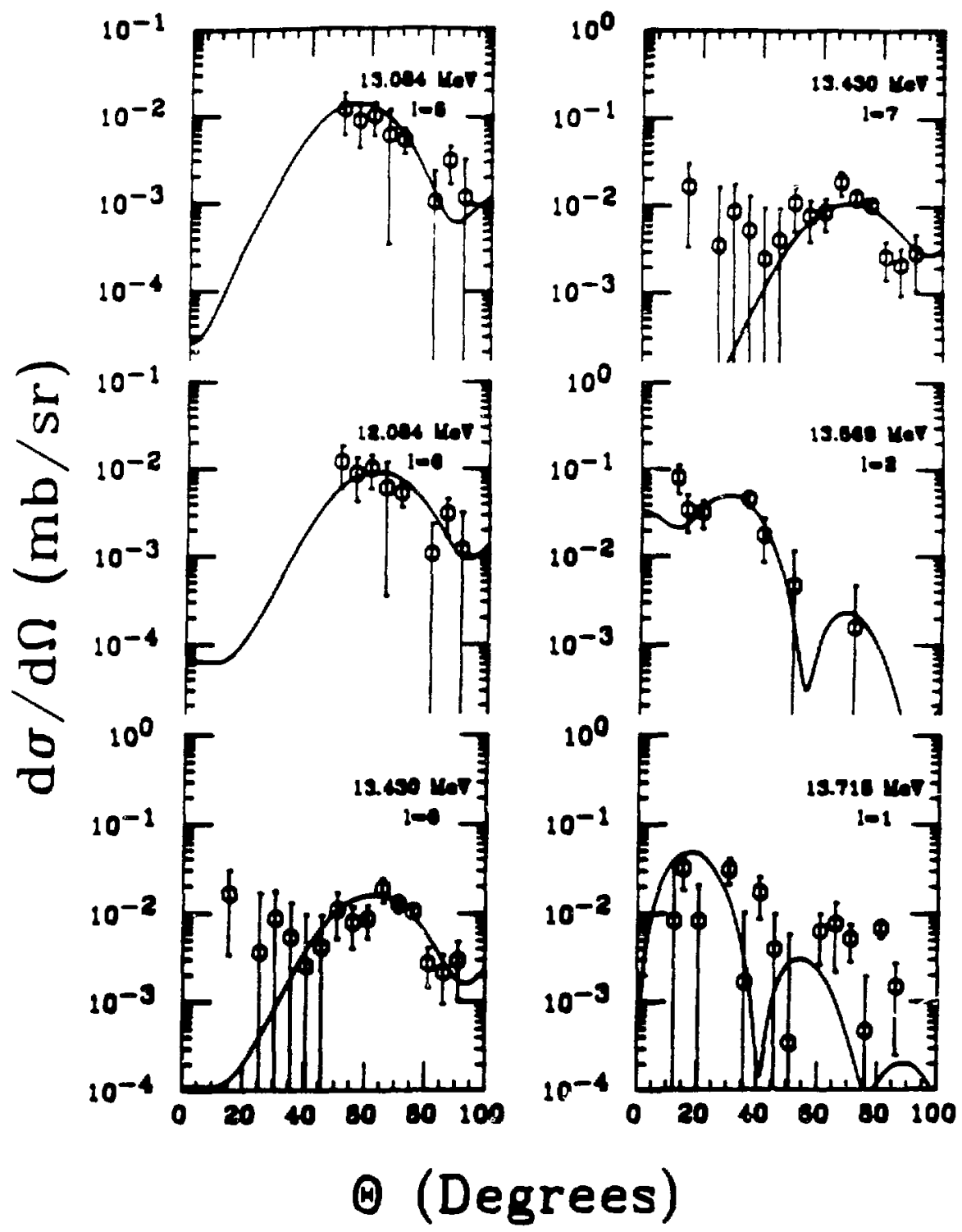

Figure A1-10.

Same as Figure A1-1 but for states between 13.08 and $13.72 \mathrm{MeV}$ in excitation. 


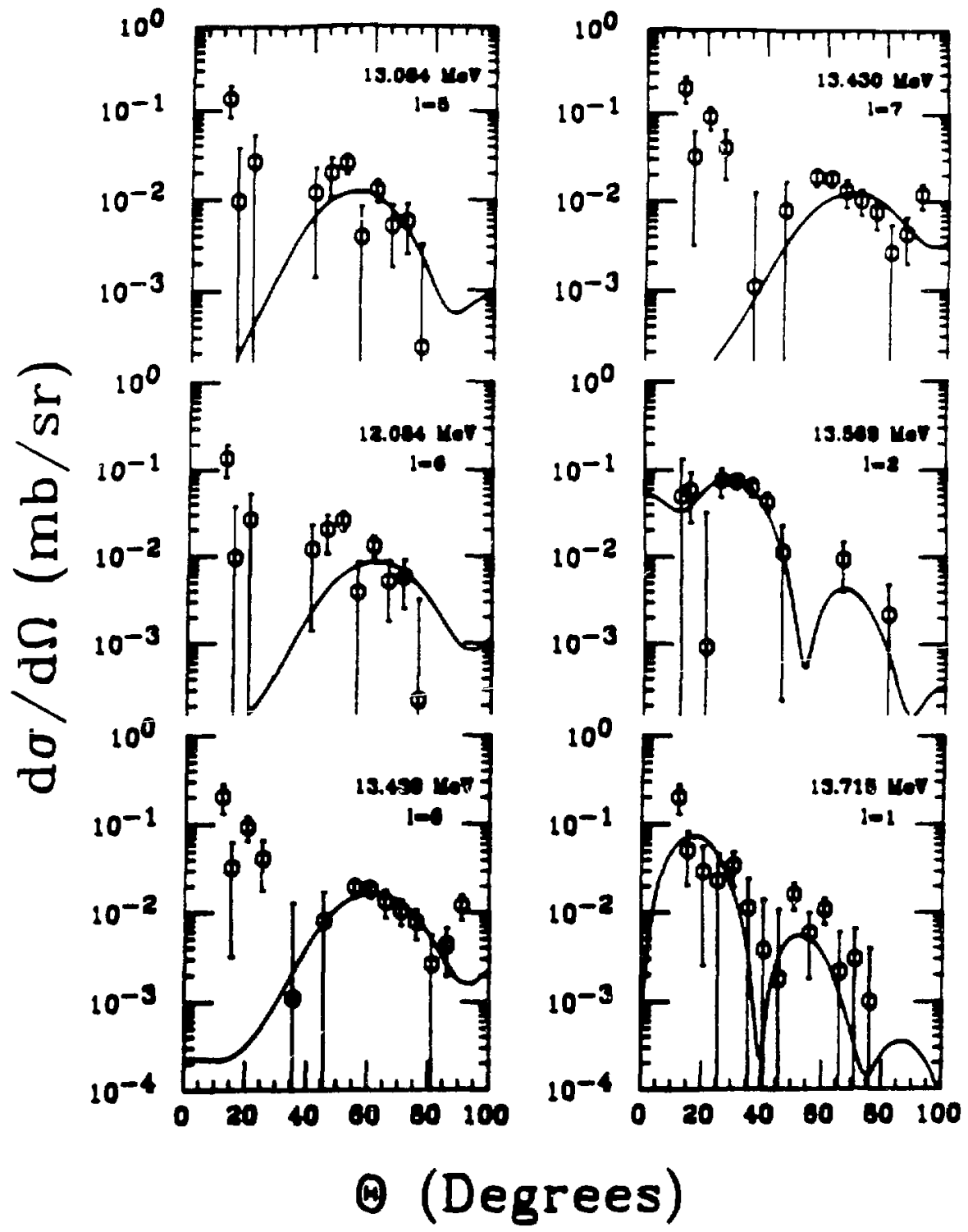

Figure A1-11.

Same as Figure A1-10 except the graphs are $180-\mathrm{MeV} \pi^{-}$dats. 


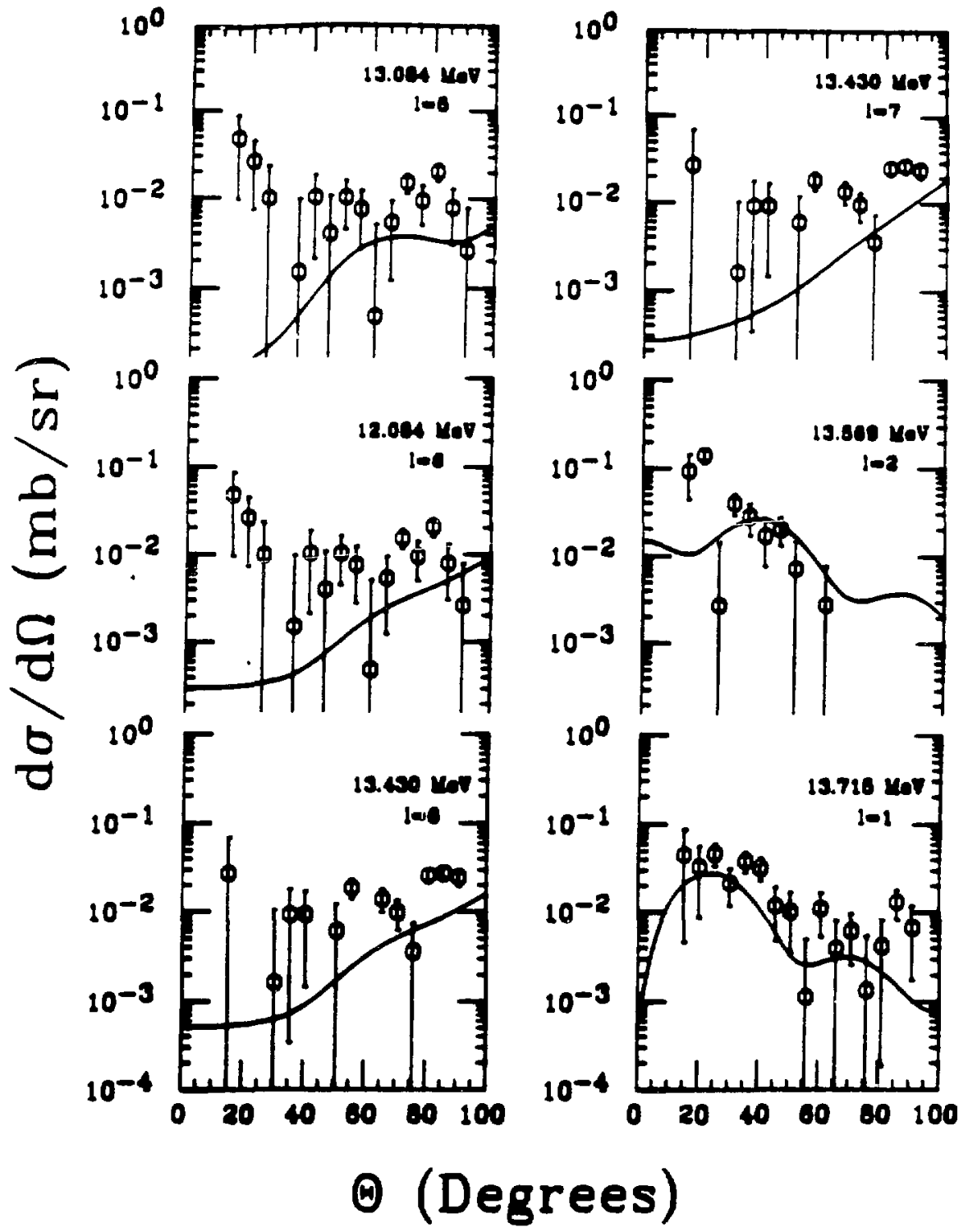

Figure A1-12.

Same as Figure A1-10 except the graphs are $120-\mathrm{MeV} \pi^{+}$data. 


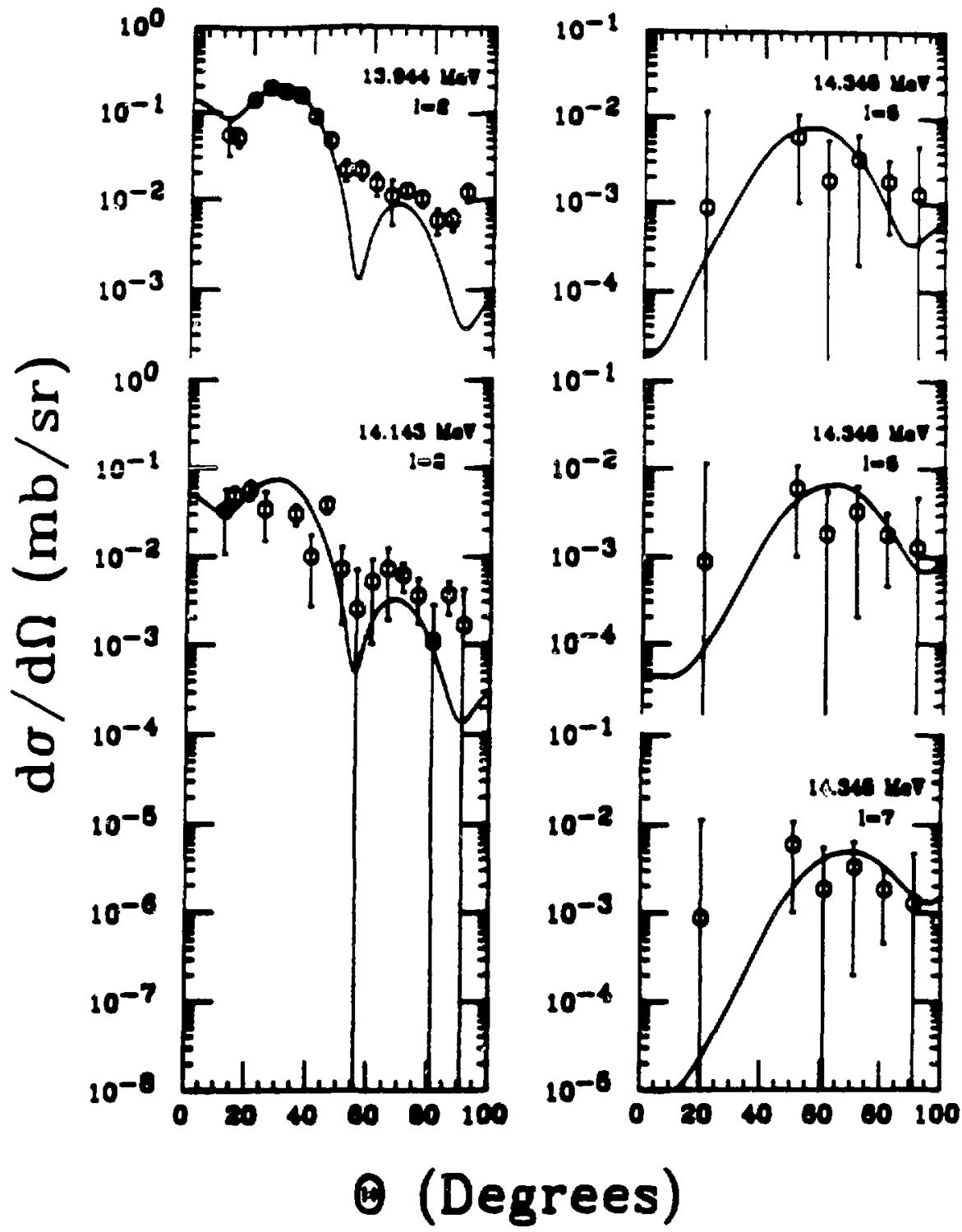

Figure A1-13.

Same as Figure A1-1 but for states between 13.94 and $14.35 \mathrm{MeV}$ in excitation. 


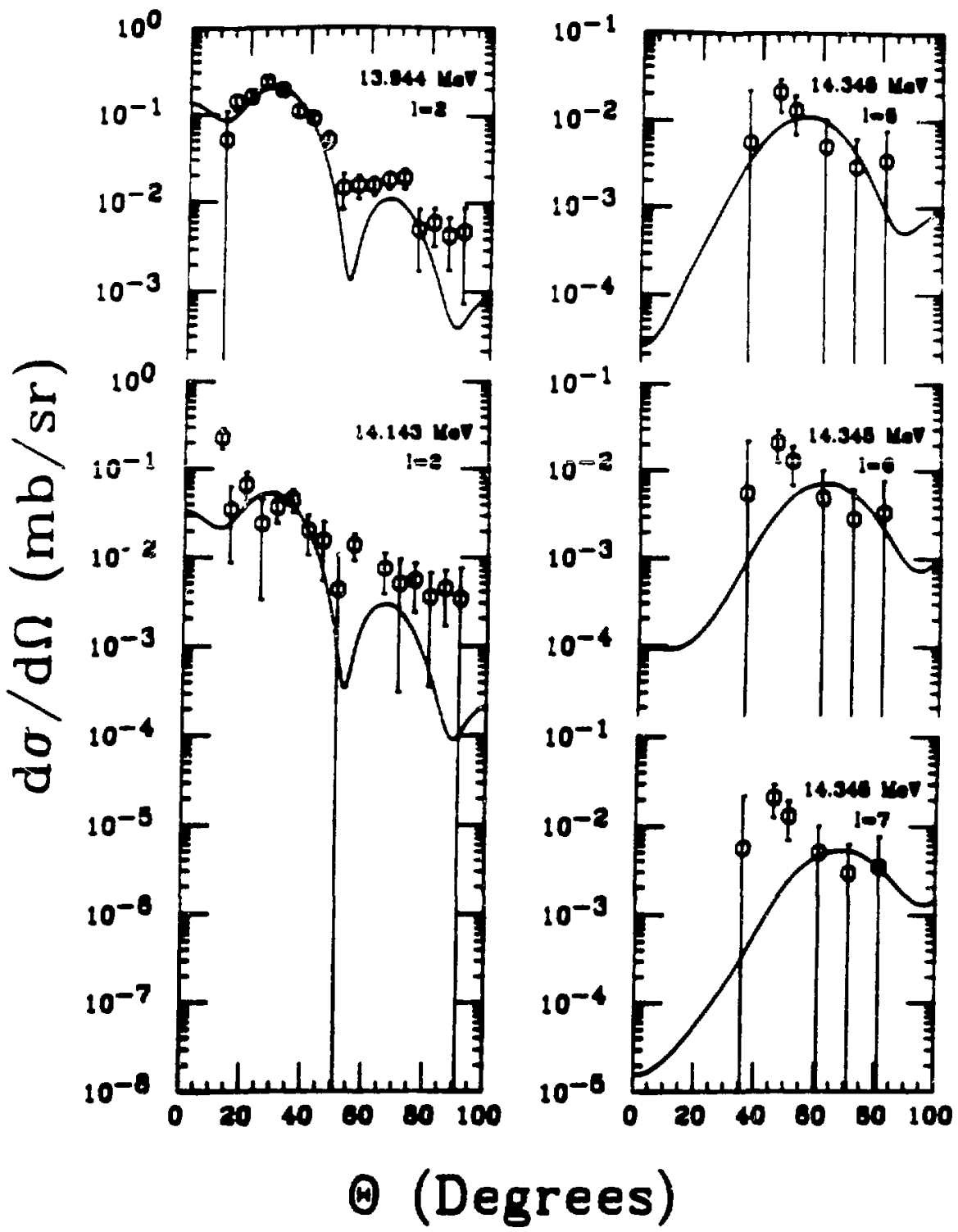

Figure A1-14.

Same as Figure A1-13 except the graphs are $180-\mathrm{MeV} \pi^{-}$data. 


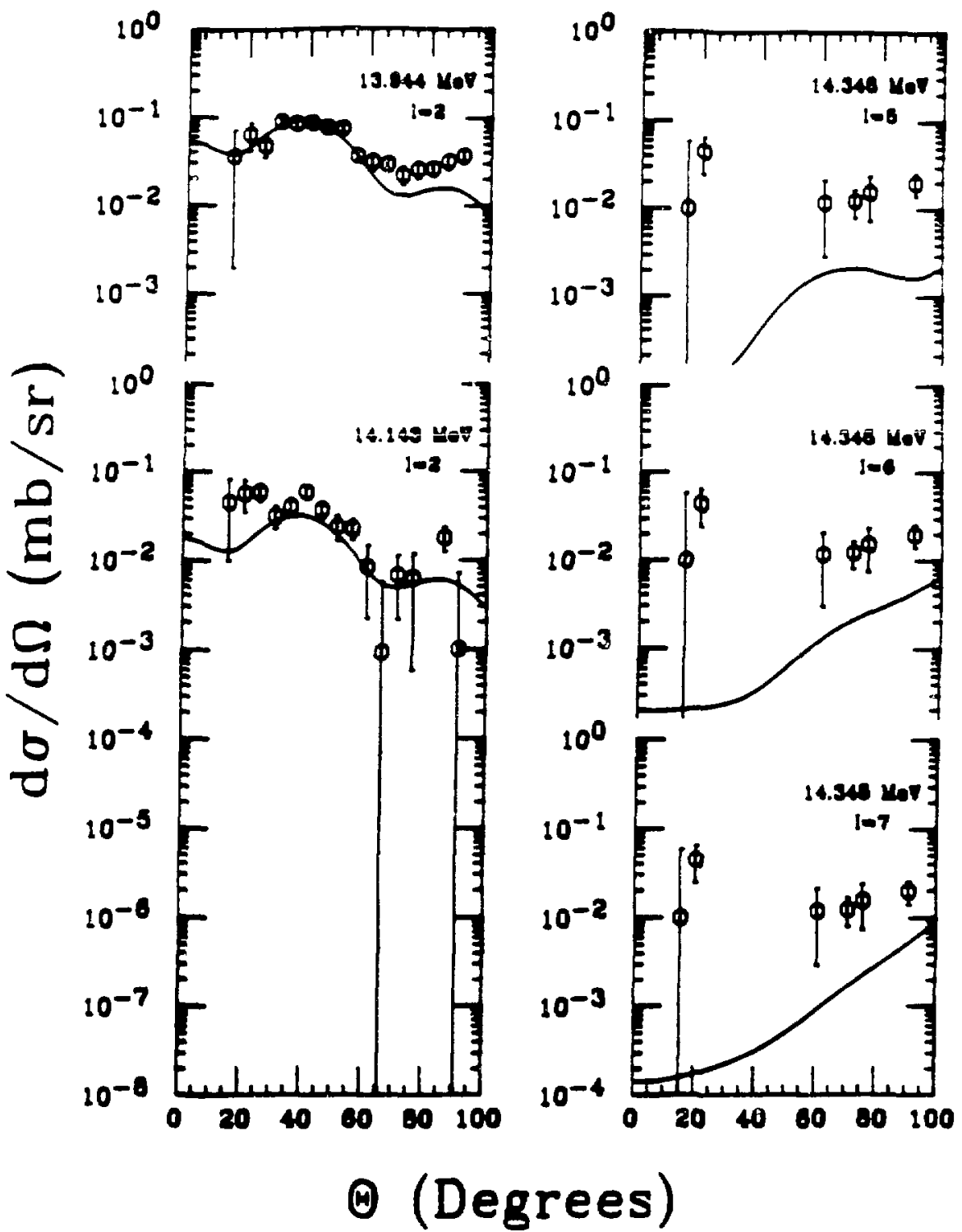

Firure A1-15.

Same as Firure Al-13 except the graphs are $120-\mathrm{MeV} \pi^{+}$data. 


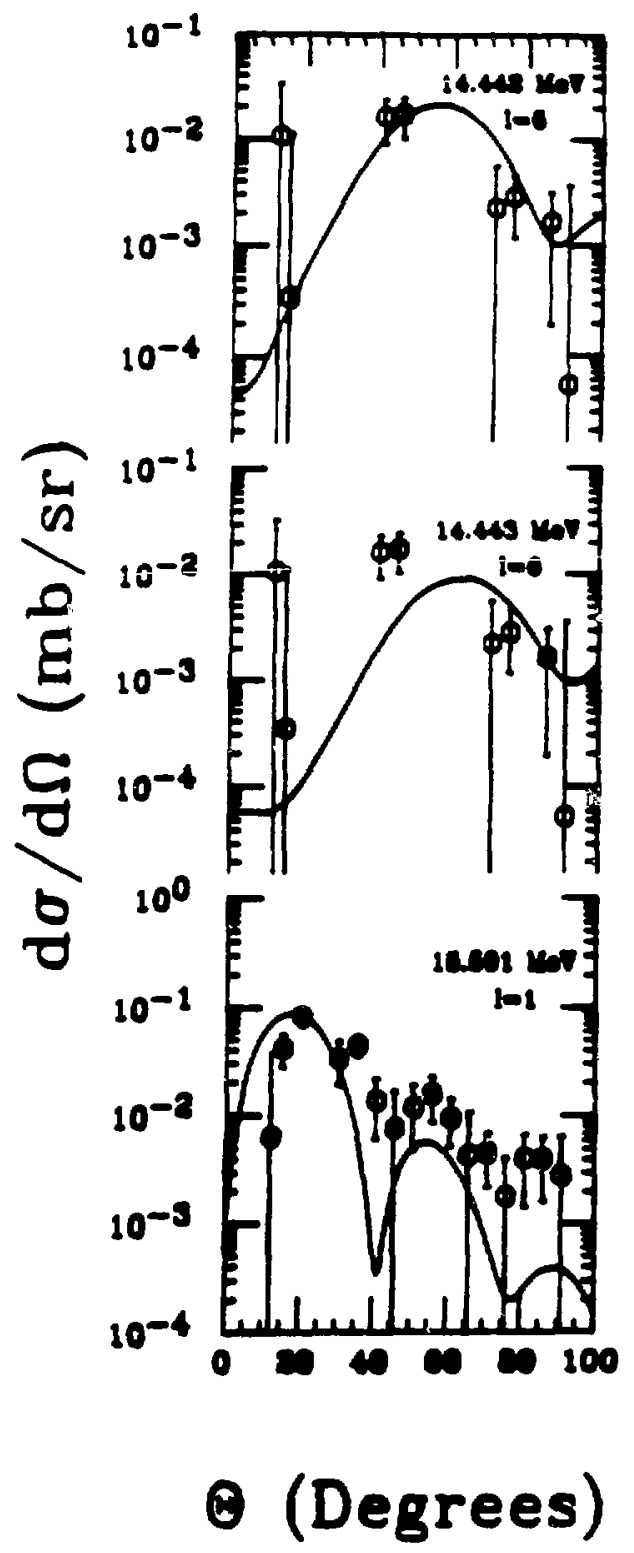

Figure A1-16.

Same an Figure Al-1 but for stated between 14.44 and $15.50 \mathrm{MeV}$ in excitation. 


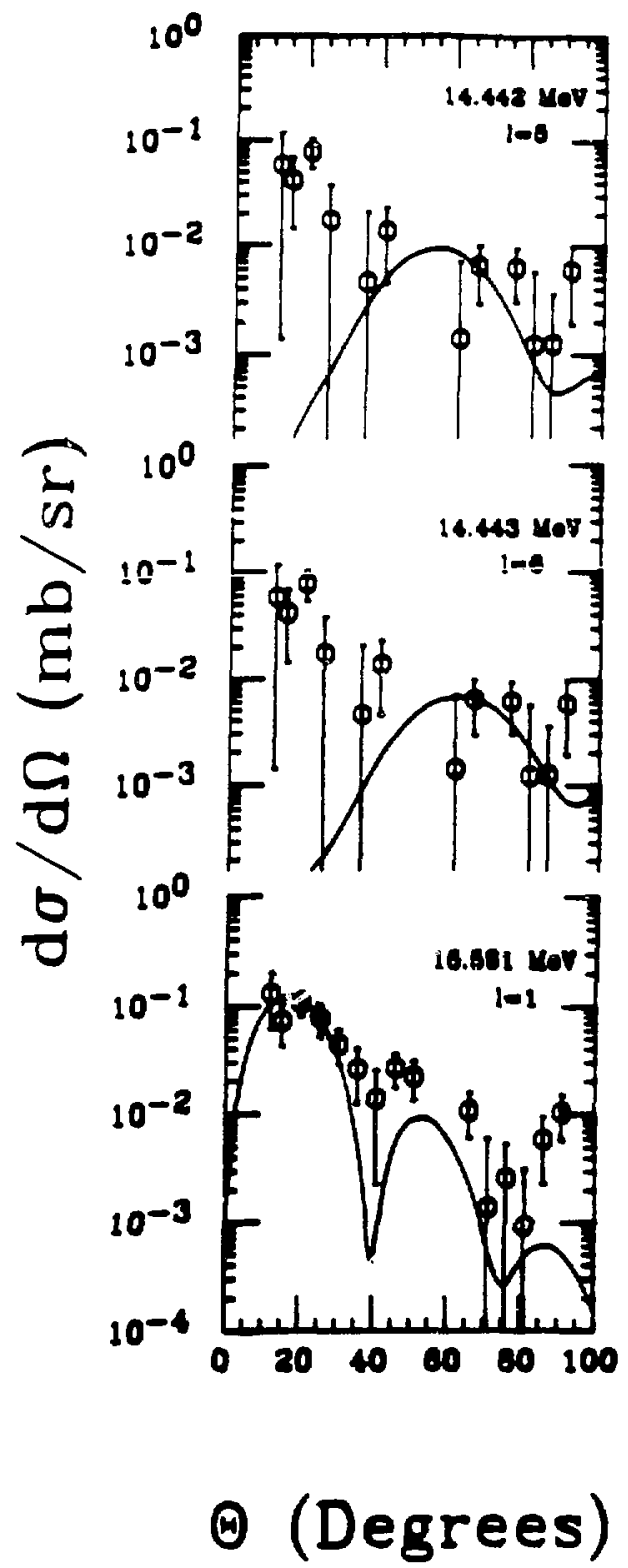

Figure A1-17.

Same as Figure A1-16 except the graphs are 180-MeV $\pi^{-}$date. 
-200 -

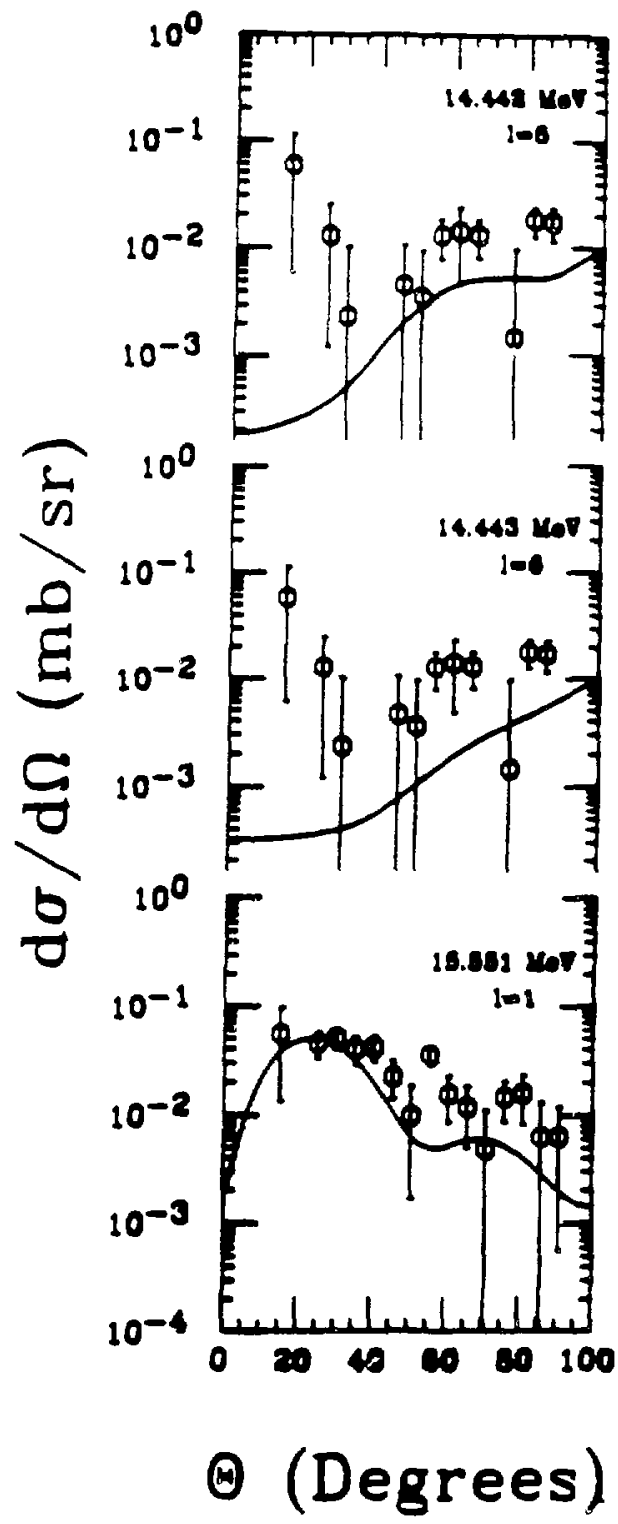

Figure A1-18.

Same as Figure Al-16 except the grepha are 120-MeV $\pi^{+}$dats. 


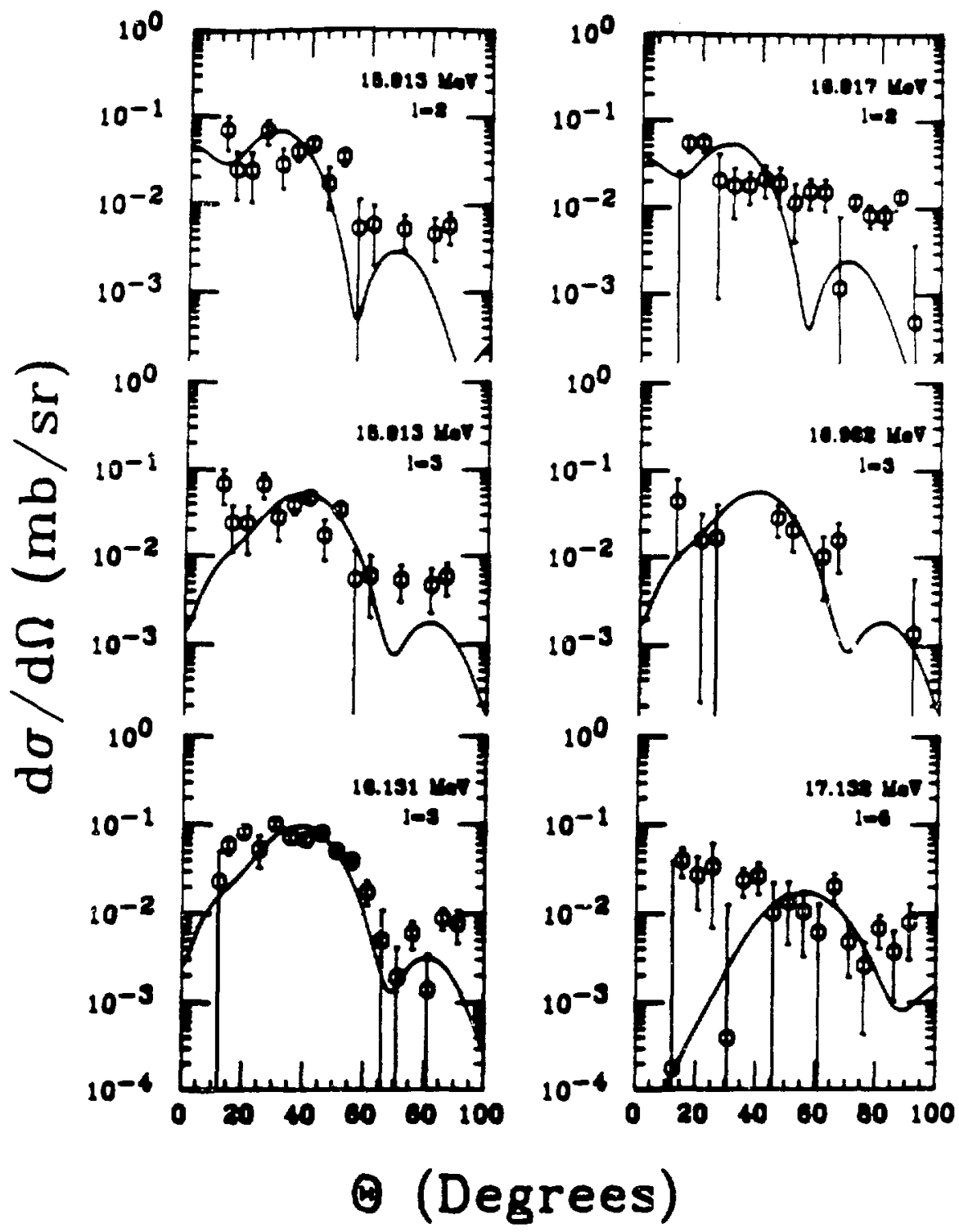

Figure A1-19.

Same as Figure Al-1 but for states between 15.91 and $17.13 \mathrm{MeV}$ in excitation. 


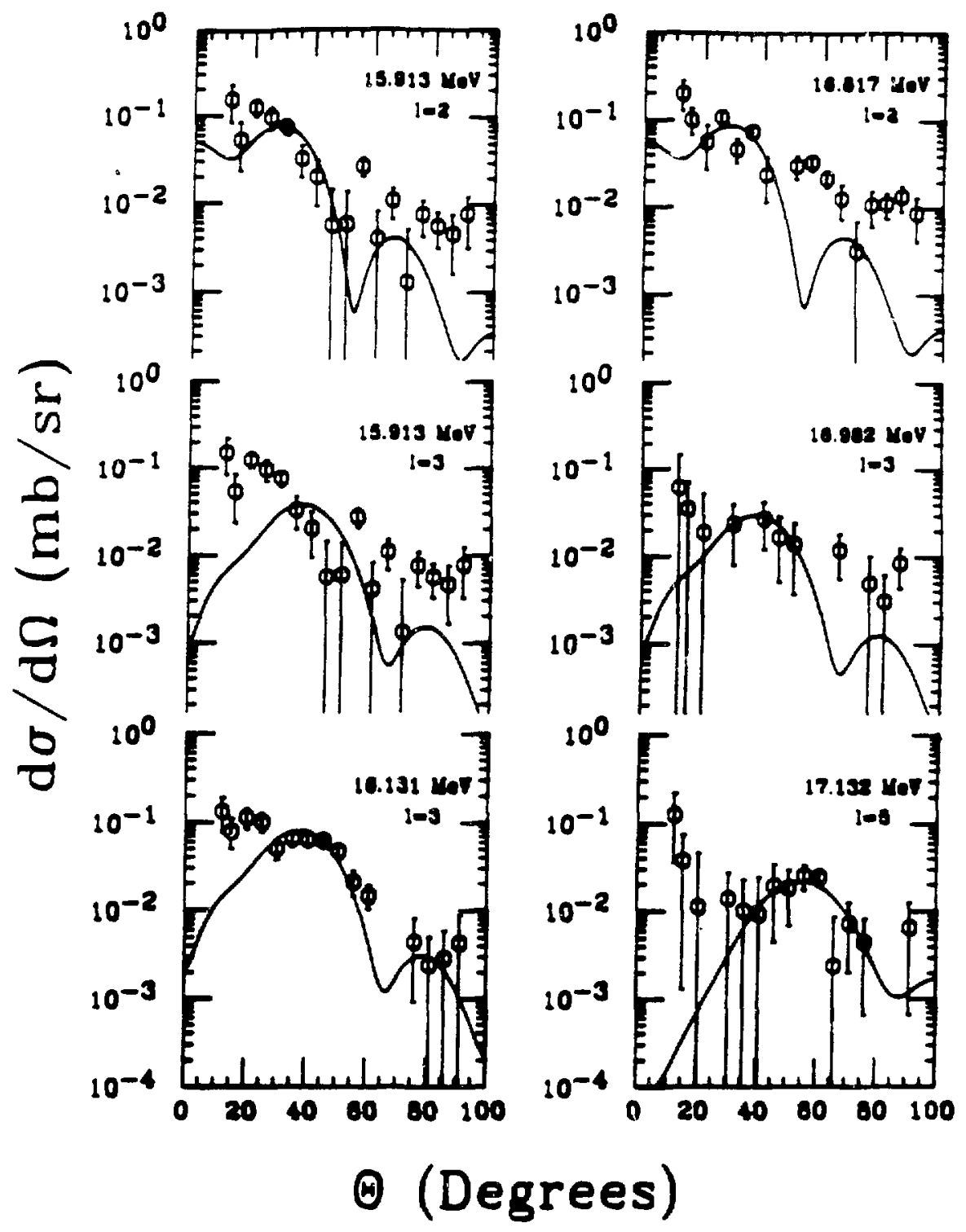

Figure A1-20.

Same as Figure Al-10 except the sraphs are $180 \mathrm{MeV} \pi^{-}$data. 


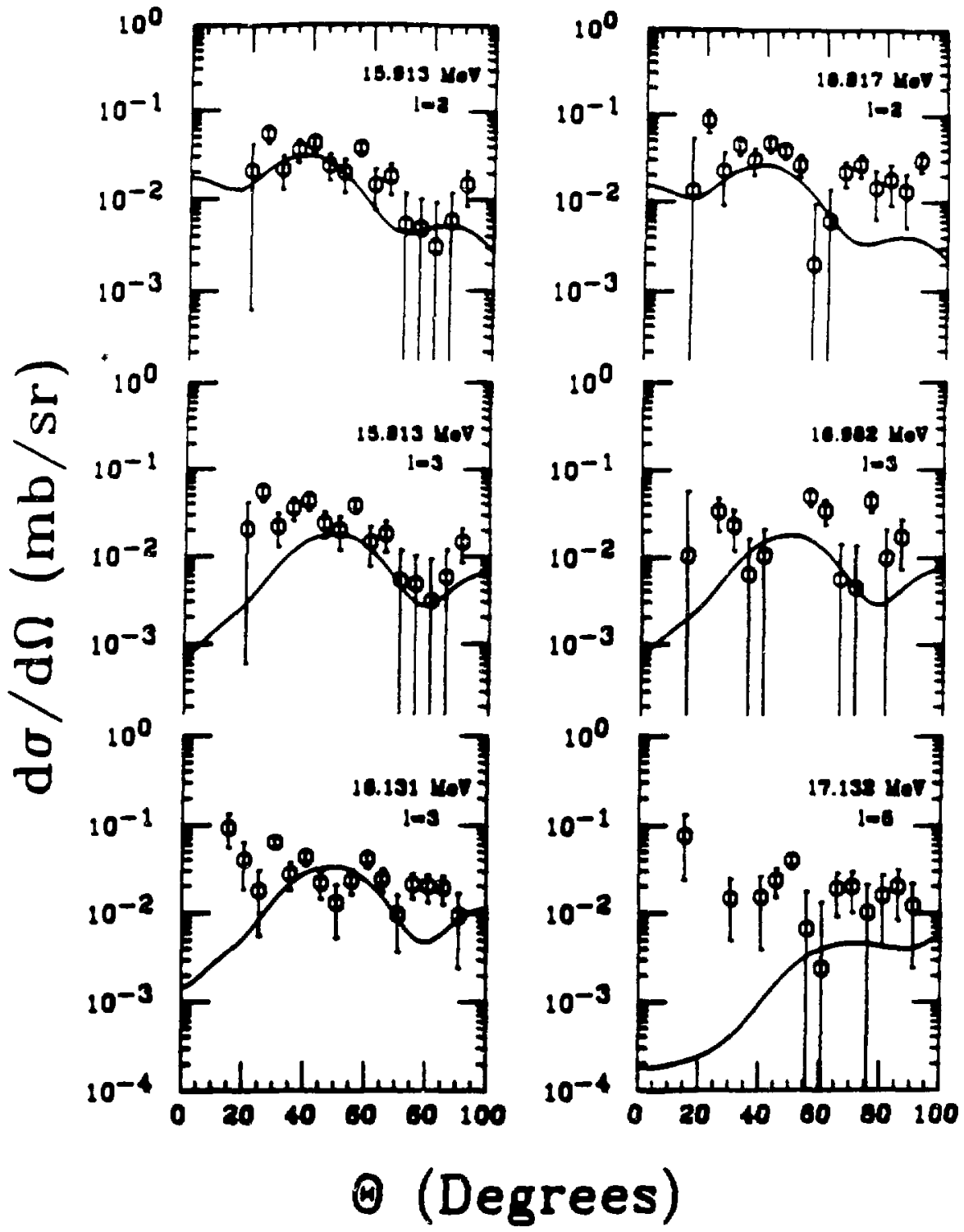

Firure A1-21.

Same as Figure A1-19 except the graphs are $120-\mathrm{MeV} \pi^{+}$data. 


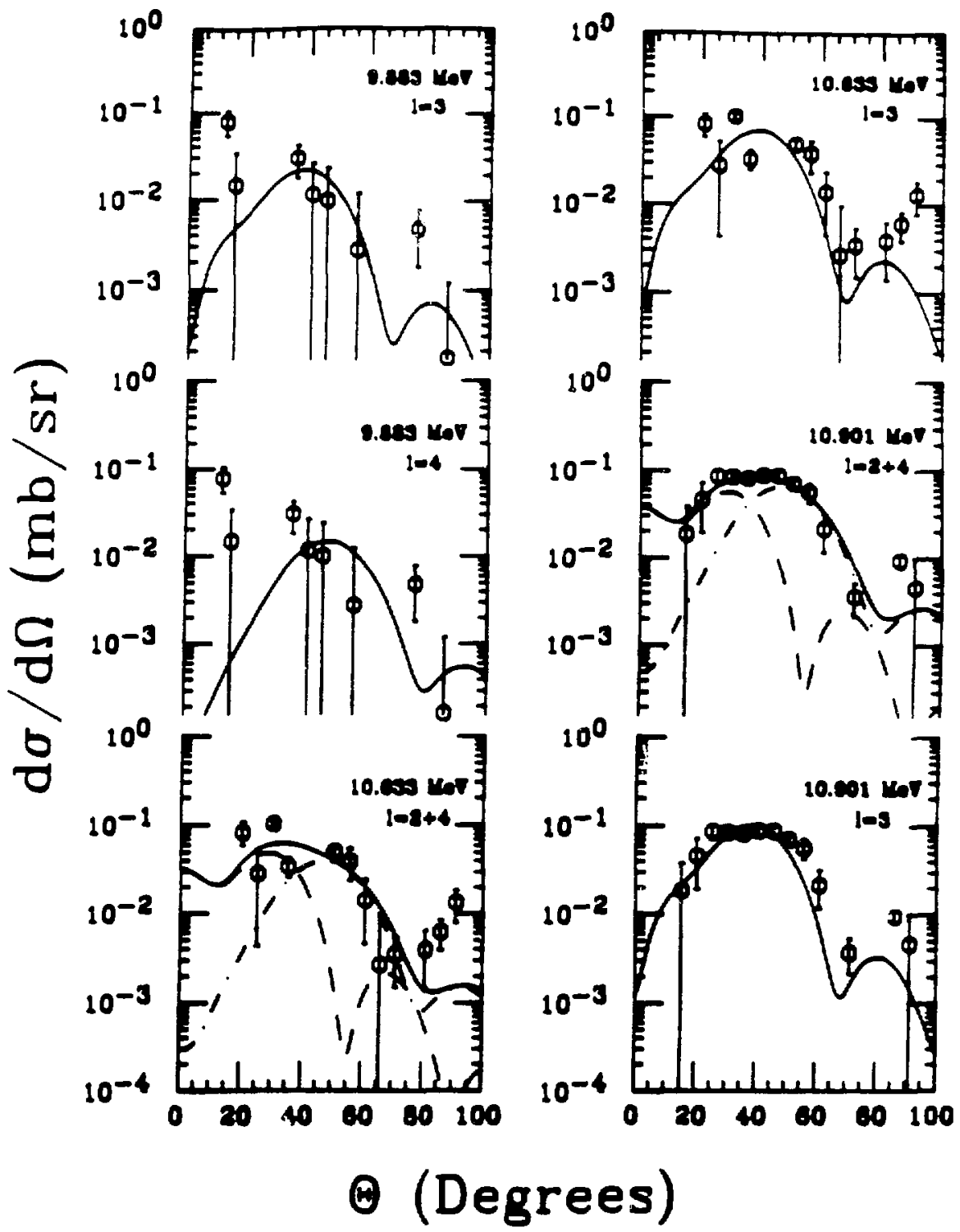

Figure A1-22.

Angular distributions for peaks between 9.88 and $10.80 \mathrm{MeV}$ in excitation which could not be well fit with a single l. All of the graphs in both columns are $180-\mathrm{MeV} \pi^{+}$. The curves are DWLA calculations using a collective transition density. For peaks fit with more than one h, the solid curre is the incoherent sum of each $/$ tranofer. The broken curves are the contribution of each $l$. 


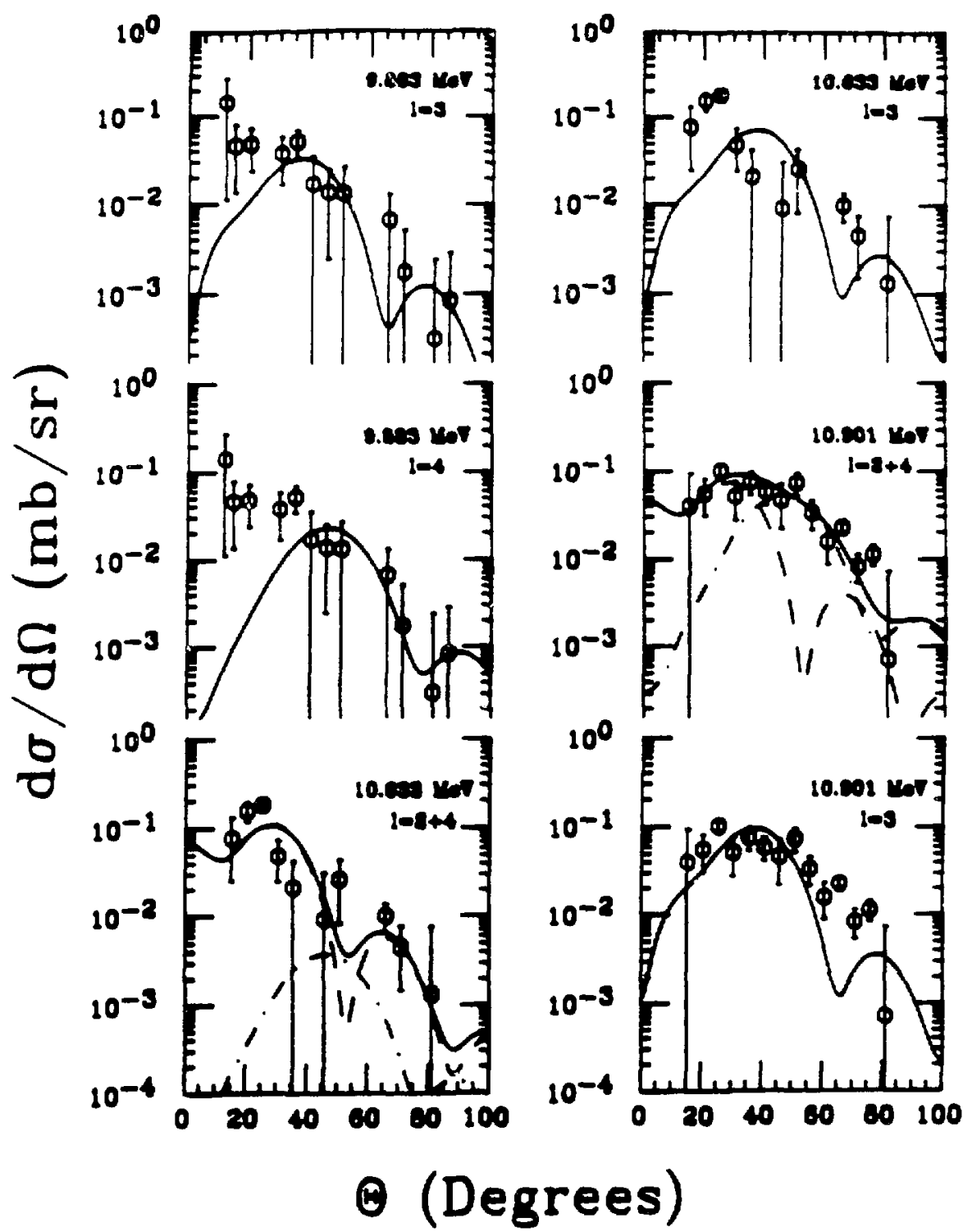

Figure A1-23.

Same as Figure A1-22 except the graphs are $180-\mathrm{MeV} \pi^{-}$dats. 


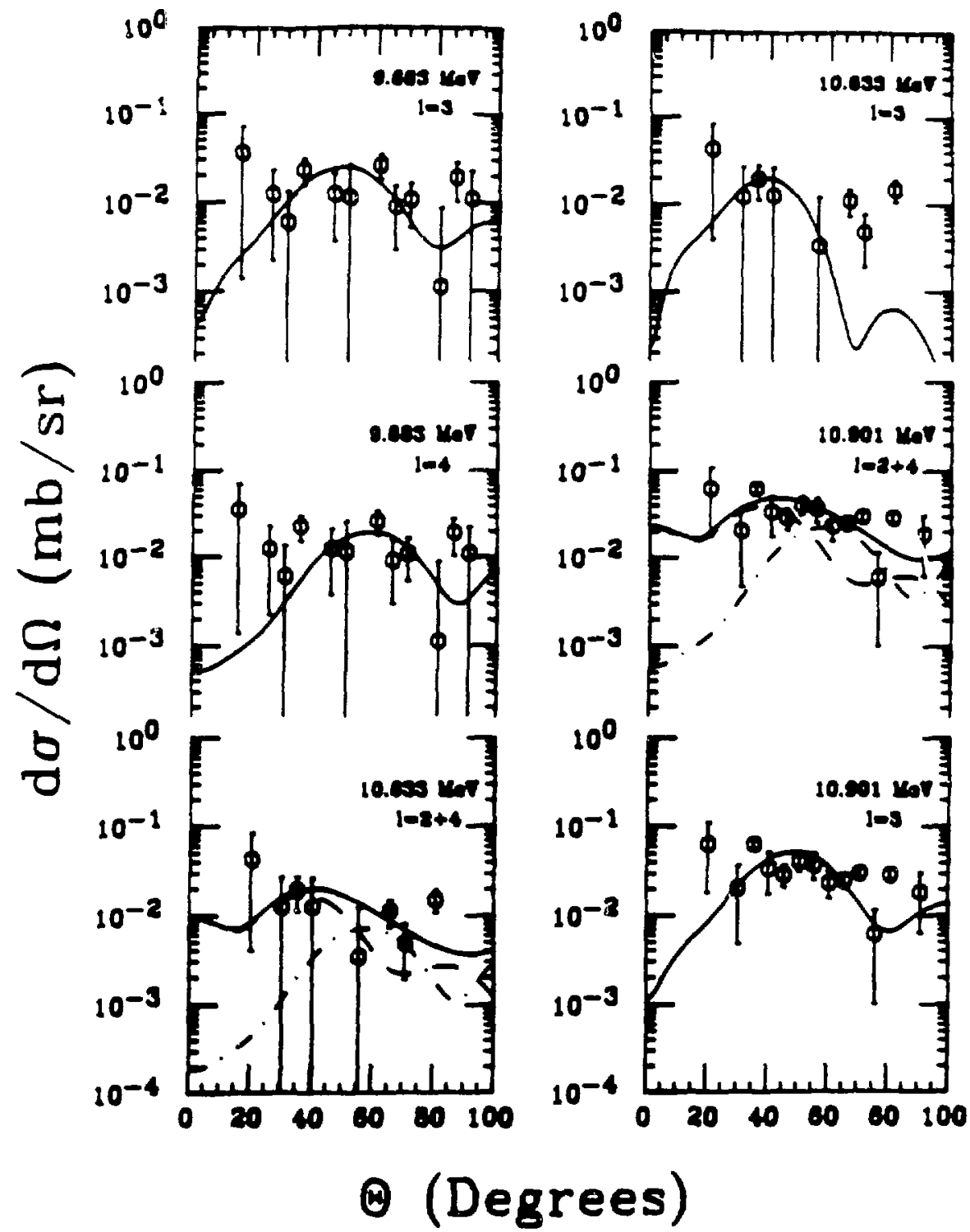

Figure A1-24.

Same as F igure A1-22 except the graphs are 120-MeV $\pi^{+}$data. 


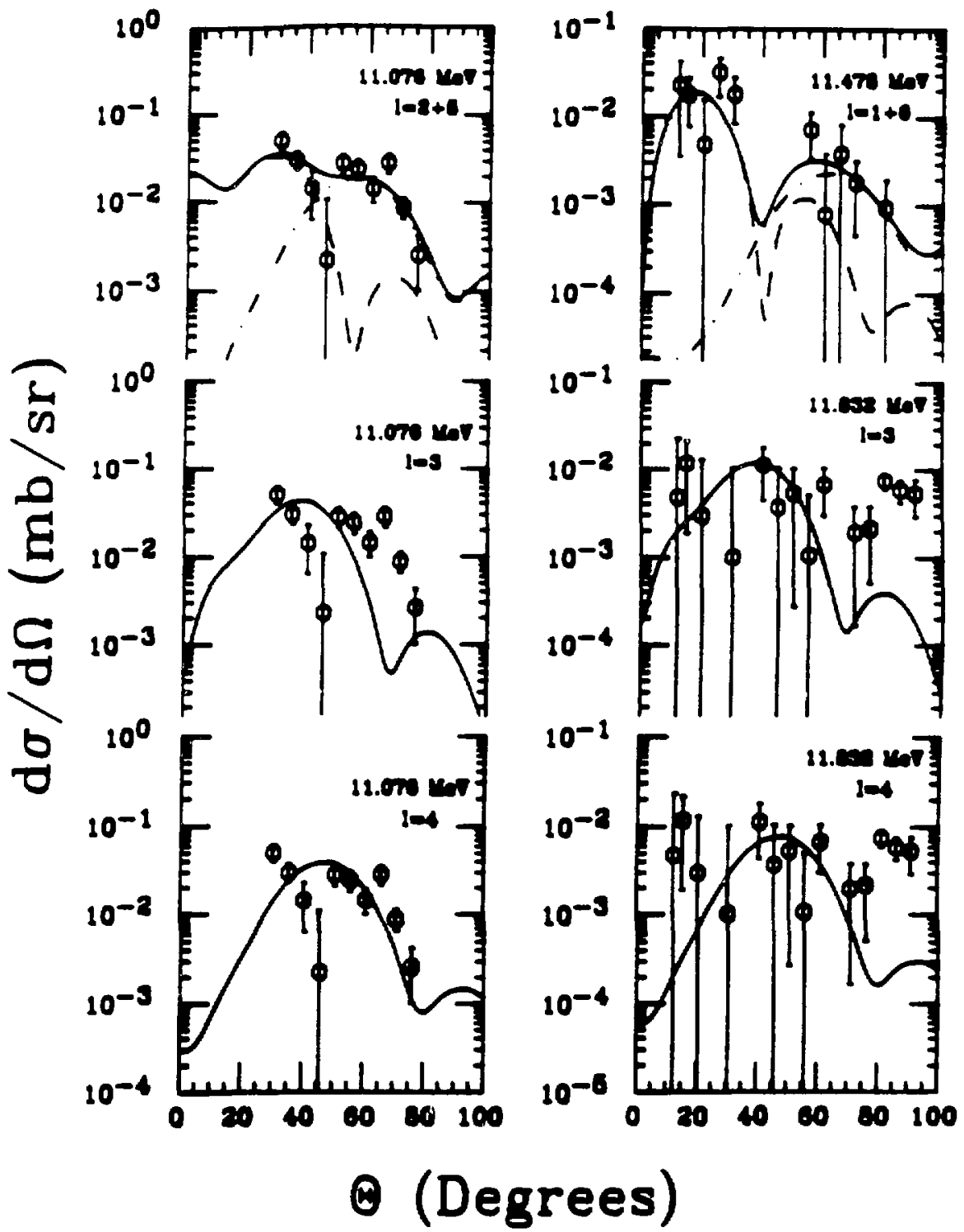

Figure A1-25.

Same as Figure A1-22 but for states between 11.08 and $11.83 \mathrm{MeV}$ in excitation. 


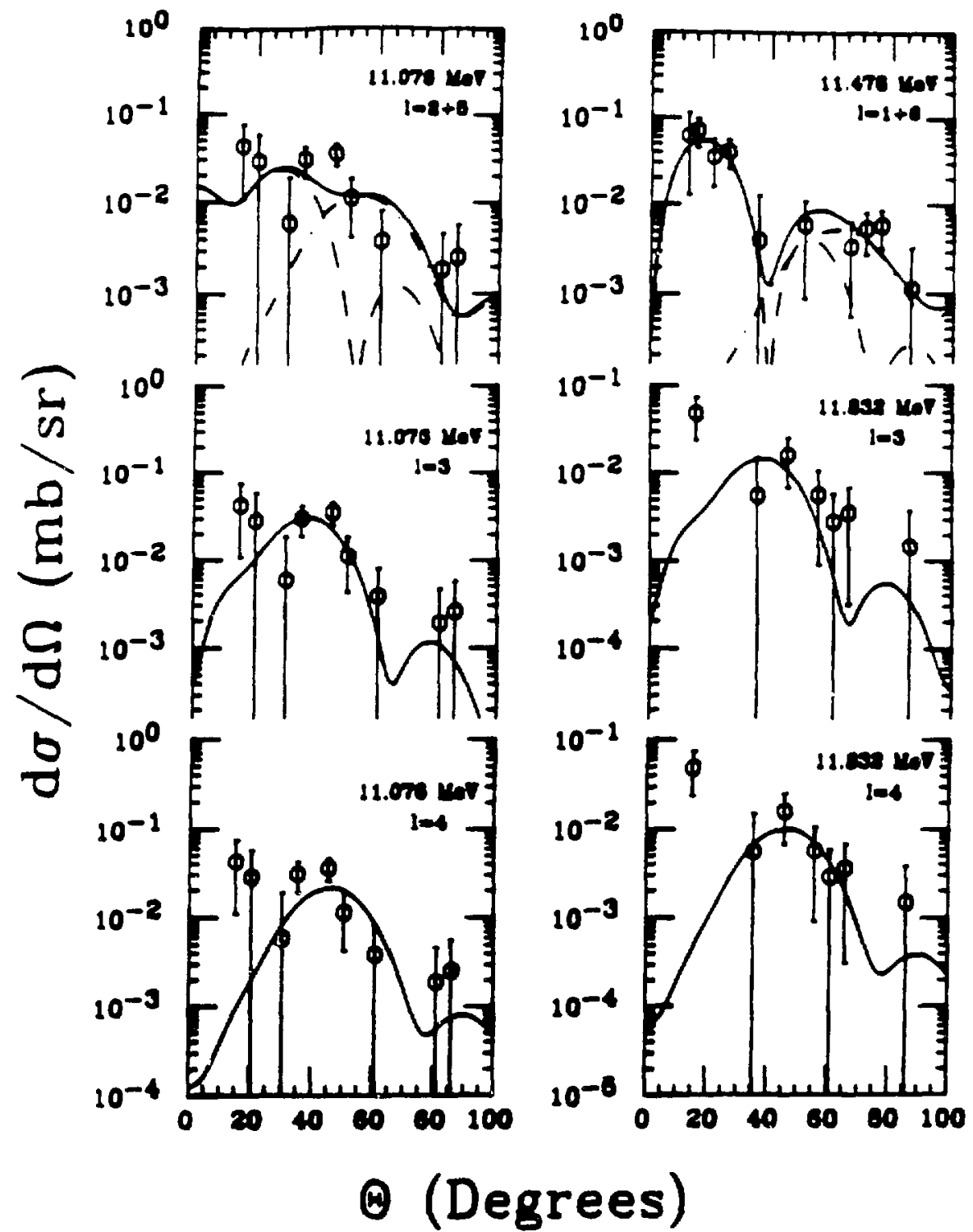

Figure A1-26.

Same as Figure A1-25 except the craphs are $180-\mathrm{MeV} \pi^{-}$data. 


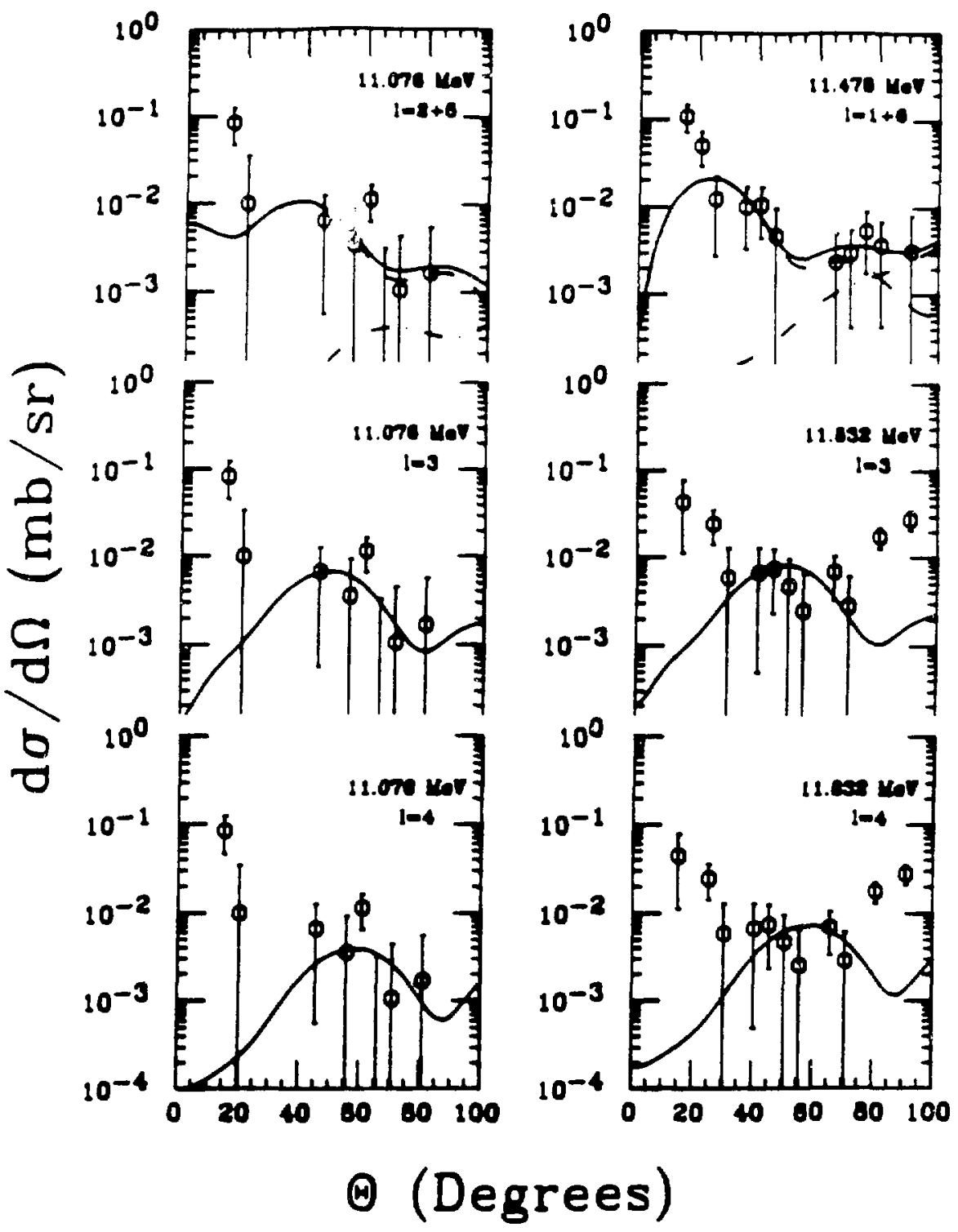

Figure A1-2\%.

Same as Figure A1-25 except the graphs are $120-\mathrm{MeV} \pi^{+}$data. 


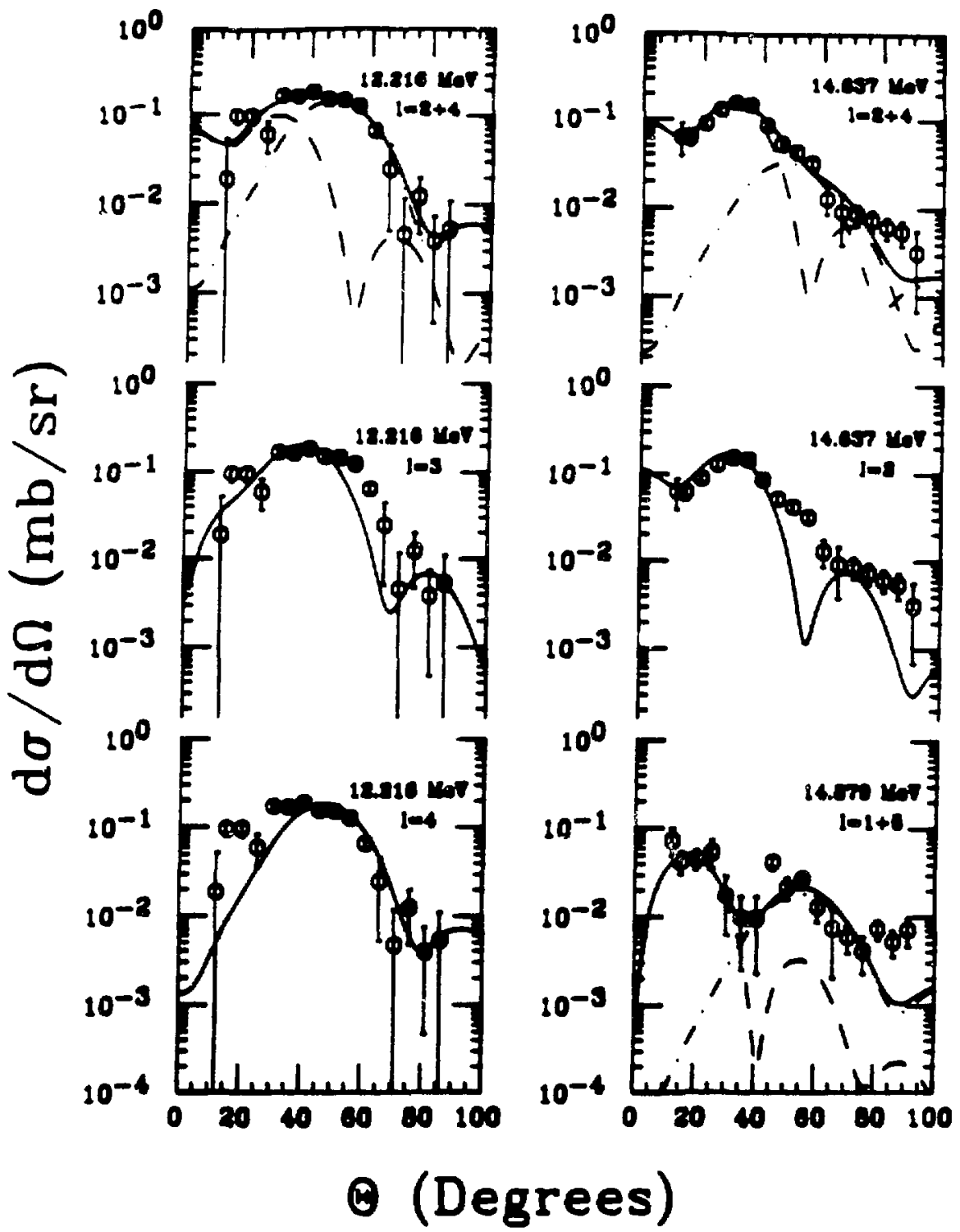

Figure A1-28.

Same as Figure Al-22 but for states between 12.22 and $14.88 \mathrm{MeV}$ in excitation. 


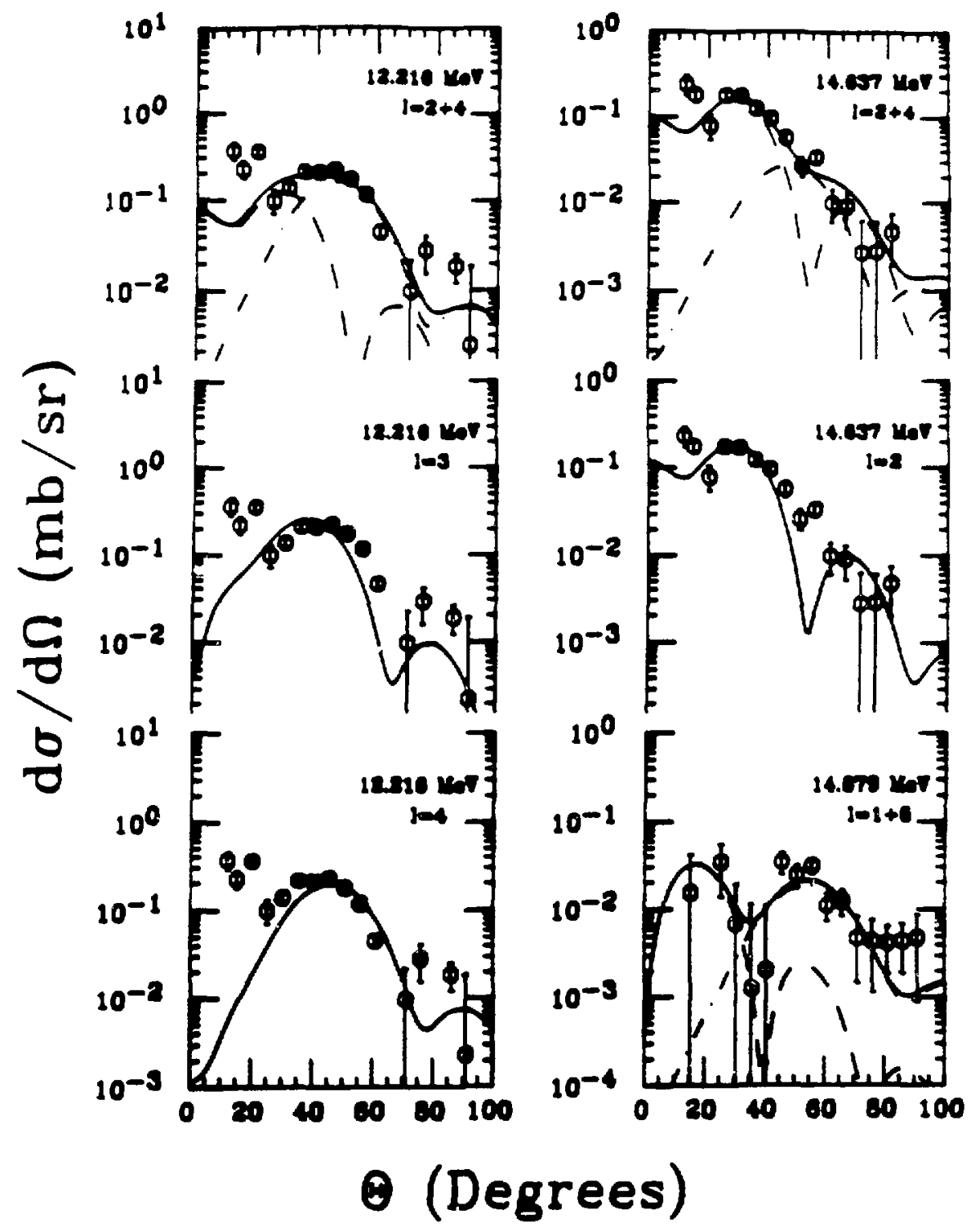

Figure Al-29.

Same as Figure A1-28 except the craphs are $180-\mathrm{MeV} \pi^{-}$data. 


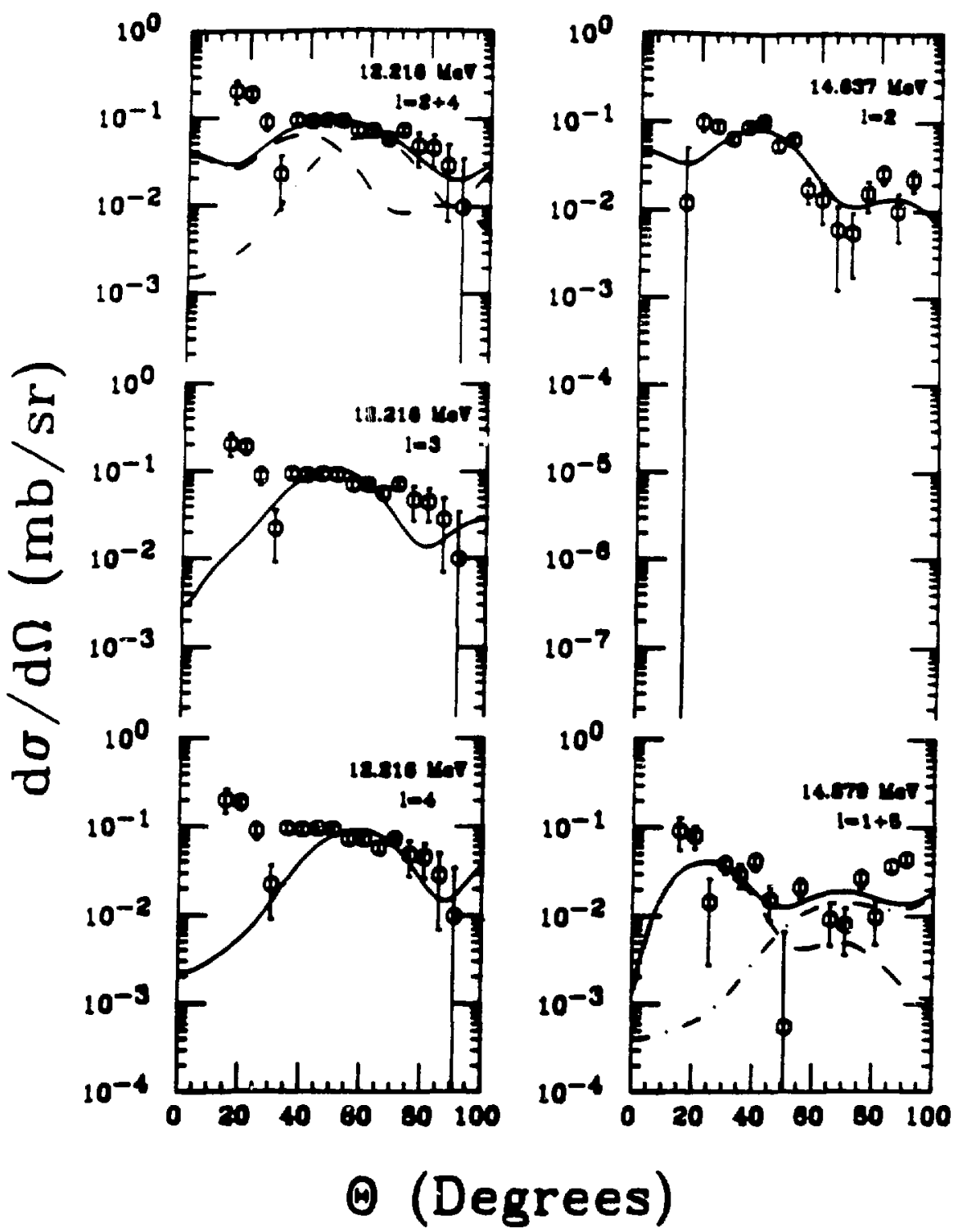

Figure A1-30.

Same as Fifure A1-28 except the graphs are $120-\mathrm{MeV} \pi^{+}$dats. 

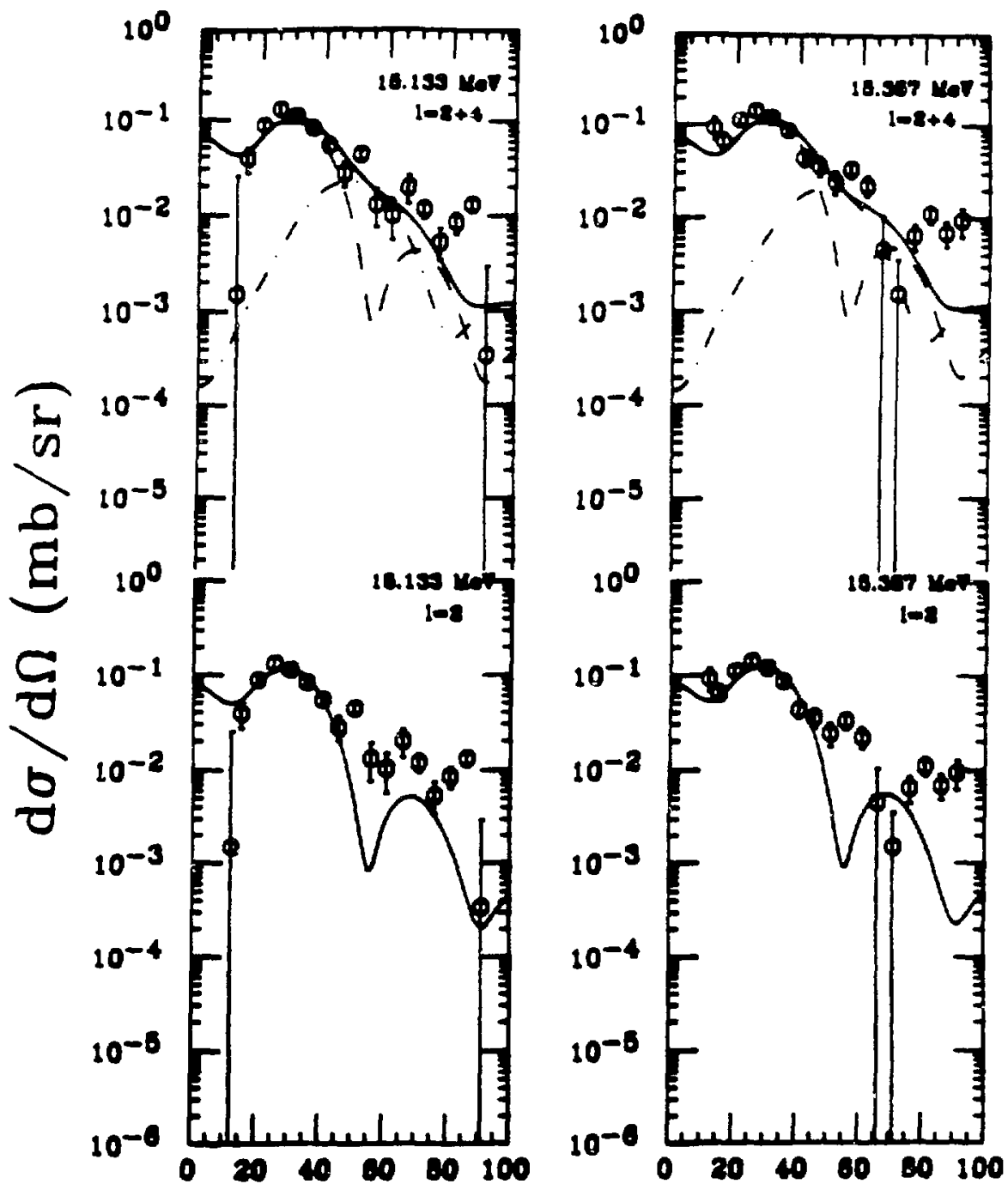

() (Degrees)

Figure A1-31.

Same as Figure Al-22 but for states between 15.13 and $15.36 \mathrm{MeV}$ in excitation. 


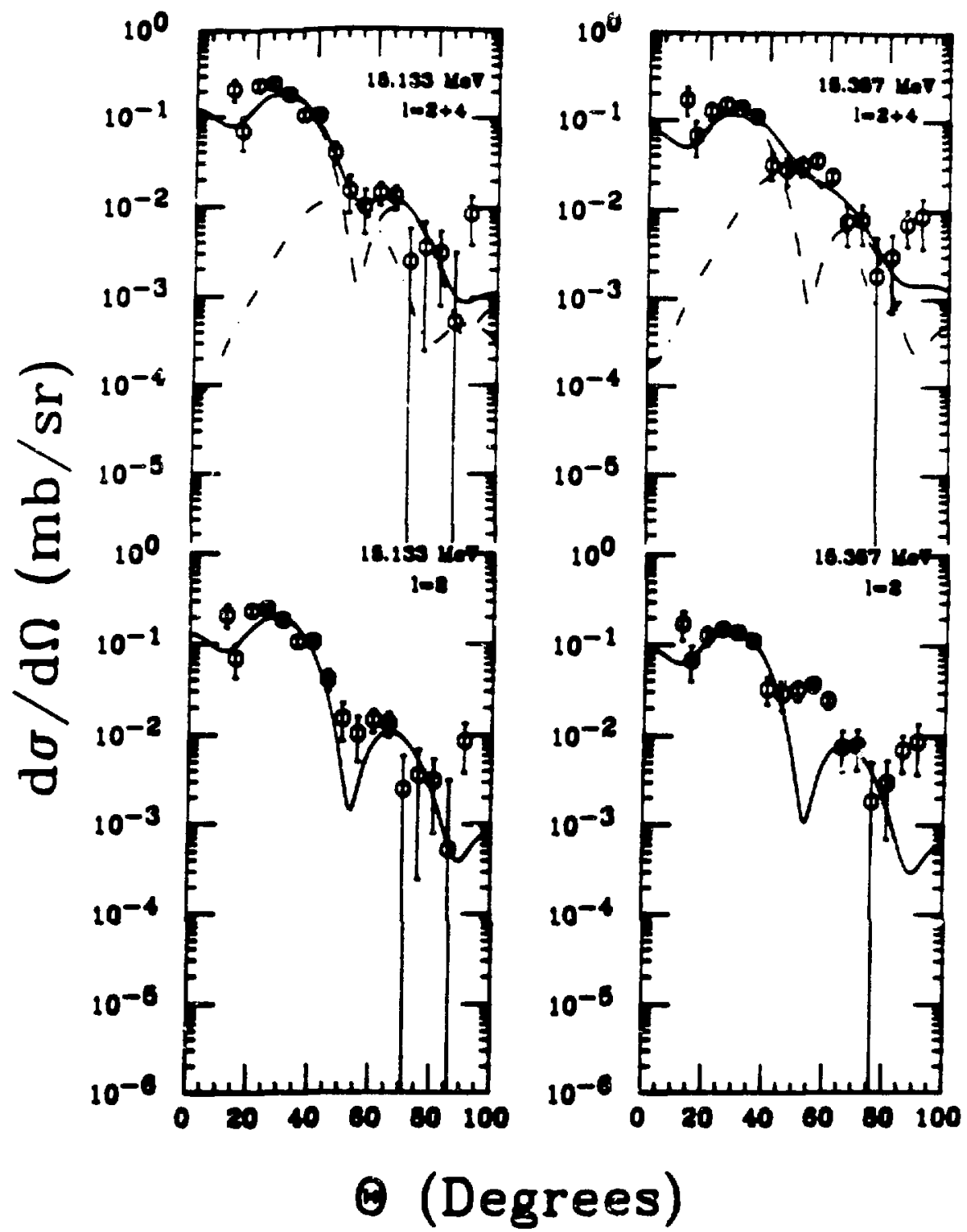

Figure A1-32.

Same an Figure Al-31 except the graphw are $180-\mathrm{MeV} \pi^{-}$data. 

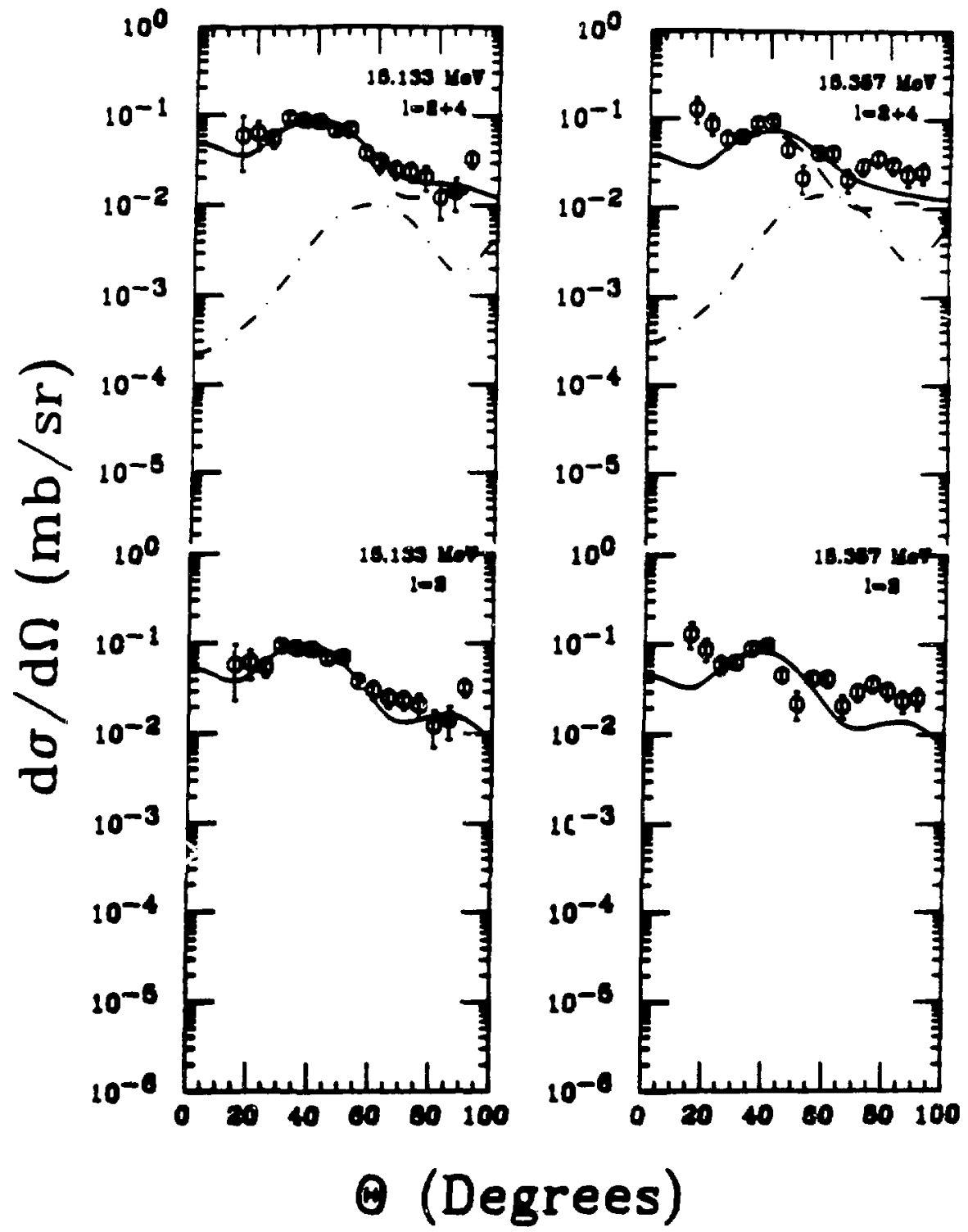

Figure A1-33.

Same as Figure A1-31 except the graphs are 120-MeV $\pi^{+}$data. 


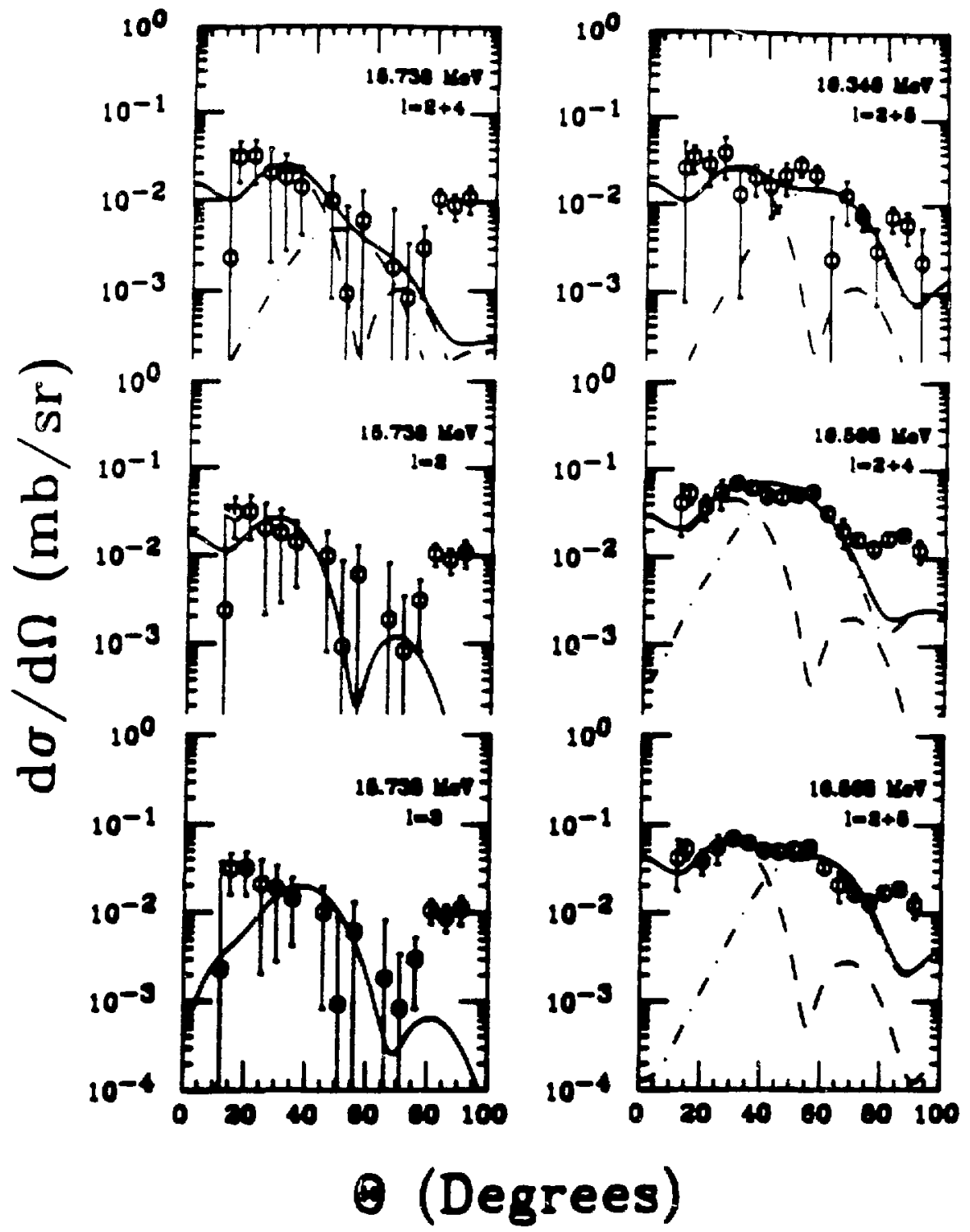

Figure A1-34.

Same as Figure A1-22 but for states between 15.74 and $16.59 \mathrm{MeV}$ in excitation. 


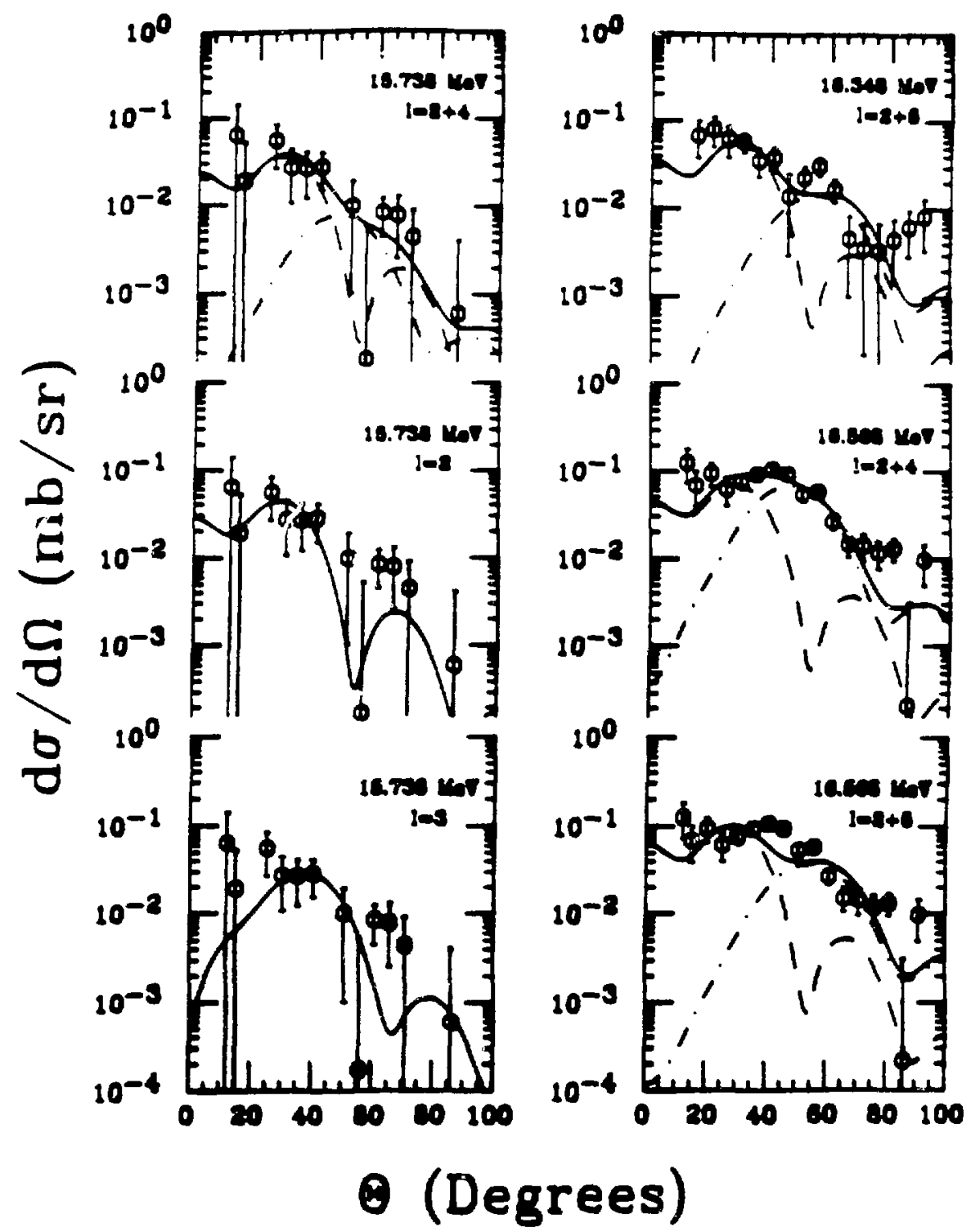

Figure A1-35.

Same as Figure A1-34 except the graphs are $180-\mathrm{MeV} \pi^{-}$data. 


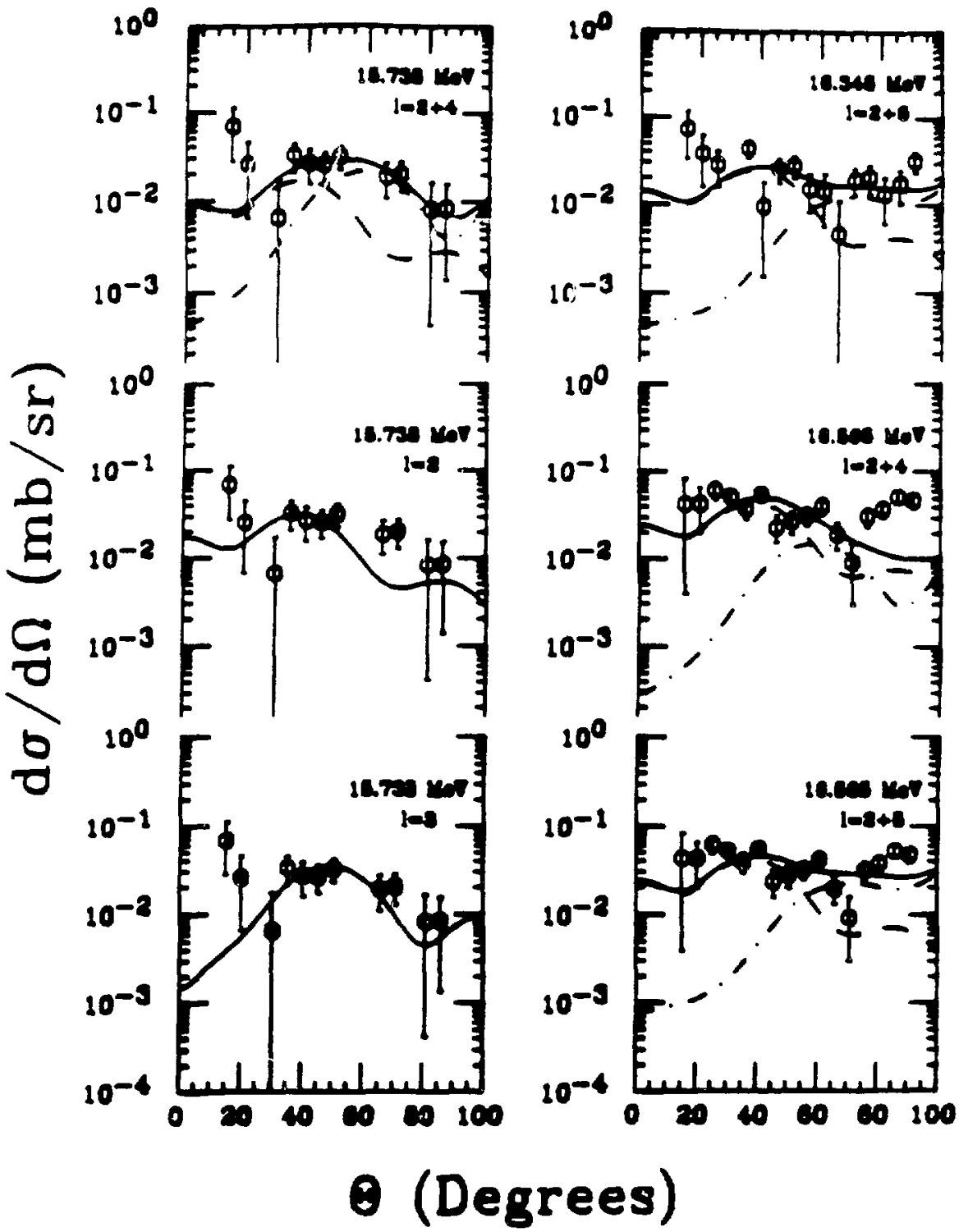

Figure A1-36.

Same as Figure A1-34 except the craphs are $120-\mathrm{MeV} \pi^{+}$data. 


\section{Appendix 2.}

Pion inelastic scattering to the low-lying broad $2+$ in ${ }^{20} \mathrm{Ne}$

The following paper, resulting from this work, has been accepted for publication in Physical Review C. 
Pion inelastic scattering to the low-lying broad $2+$ in ${ }^{20} \mathrm{Ne}$

M. Burlein, H.T. Fortune, W. M. Amos, T. L. Ekenberg, A. Kotwal, P. H. Kutt,

J. M. O'Donnell, and J. D. Silk

University of Pennoylvanis

Philadelphia, PA 19104

B. Boyer, A. Fuentes, K. Johnson, C. F. Moore, and S. H. Yoo

University of Texas at Austin

Austin, TX 78712

S. Mordechai

University of Texse at Austin

Austin, TX 78712

and

Ben-Gurion University of the Neger

Beer-Shera 84108, larael

C. L. Morris

Los Alamos National Laboratory

Lon Alemos, NM 87545

J. D. Zumbro

University of Pennoylvania

Philadelphis, PA 19104

and

Los Alamos National Laboratory

Los Alemos, NM 87545

D. L. Watson

University of York

York Y01-5DD, United Kingdom

and

Los Alamos National Laboratory

Los Alemos, NM 87545

K. S. Dhuga'

New Mexico State University

Los Cruces, NM 88003

\section{ABSTRACT}

We have observed the broad $2^{+}$member of the $\mathrm{K}^{*}=\mathrm{O}_{4}^{+}$band of ${ }^{30} \mathrm{Ne}$ with the ${ }^{20} \mathrm{Ne}\left(\pi^{+}, \pi^{+\prime}\right)$ reaction at $\mathrm{T}_{\pi}=120 \mathrm{MeV}$ and $180 \mathrm{MeV}$ and with ${ }^{30} \mathrm{Ne}\left(\pi^{-}, \pi^{-}\right)$at $\mathrm{T}_{\pi}=180 \mathrm{MeV}$. We find an excitation energy of $9.00 \pm 0.18 \mathrm{MeV}$ and a width of $0.8 \mathrm{MeV}$. The $\mathrm{B}(\mathrm{E2})$ is determined to be approximately 25 to $35 \mathrm{e}^{2} \mathrm{fm}$. 
The fourth $\mathrm{K}^{\pi}=0^{+}$band in ${ }^{20} \mathrm{Ne}$ is very difficult to study experimentally. The first two members in this band have been observed in only two reactions ${ }^{1}$ - as resonances in ${ }^{10} \mathrm{O}(\alpha, \alpha)$ and as final states in ${ }^{10} \mathrm{~F}\left({ }^{3} \mathrm{He}, \mathrm{d}\right)$. These results are given in Table I, along with the results of our experiment, ${ }^{20} \mathrm{Ne}\left(\pi, \pi^{\prime}\right)$. The states in this band are believed to be either an $(f p)^{4}$ or $(g d)^{2}(f p)^{2}$ excitation and they all have large reduced widths for $\alpha$ decay - giving them their large natural widths, but making them very difficult to observe. We have performed ${ }^{20} \mathrm{Ne}\left(\pi^{+}, \pi^{+\prime}\right)$ at $T_{\pi}=120 \mathrm{MeV}$ and $180 \mathrm{MeV}$ and ${ }^{20} \mathrm{Ne}\left(\pi^{-}, \pi^{-\prime}\right)$ at $T_{\pi}=180 \mathrm{MeV}$ and we see the $2^{+}$ member of the band.

The data were taken at the Energetic Pion Channel and Spectrometer (EPICS) at the Clinton P. Anderson Meson Physics Facility (LAMPF). The target material was ${ }^{20} \mathrm{Ne}$ gas enriched to $>99.9 \%$ purity. The target was kept in a cylindrical steel gas cell at $138 \mathrm{kPa}$ and cooled to $45 \mathrm{~K}$ giving an effective areal density of $\approx 100 \mathrm{mg} / \mathrm{cm}^{2}$ in the middle of the gas cell. Data were taken at several angles for both $\pi^{+}$and $\pi^{-}$with an empty target to determine the contribution of the gas cell to the spectra. The resolution was $180 \mathrm{keV}$ full width at half maximurn. Absolute normalizations, as a function of angle, were obtained by filling the gas cell with $\mathrm{H}_{2}$ gas, measuring $\pi-p$ scattering, and comparing the yields with cross sections calculated from the $\pi$-nucleon phase shifts of Rowe, Salomon, and andau ${ }^{5}$. Relative normalizations are believed to be known to within $4 \%$ and absolute normalizations to within $10 \%$.

Figure 1 exhibits a $T_{\pi}=180 \mathrm{MeV}{ }^{20} \mathrm{Ne}\left(\pi^{+}, \pi^{+\prime}\right)$ spectrum at $30^{\circ}$, near the peak of a $2^{+}$angular distribution. The broad state near $9 \mathrm{MeV}$ is evident, with several narrow peaks on top of it. This spectrum was fitted using a linear background (dashed line) and constraining the peak energies at their known values for states in ${ }^{20} \mathrm{Ne}$. The peak shape for the narrow states was determined by fitting the 
elastic peak shape. The areas of the peaks were the only parameters allowed to vary. The peak shape for the broad state was constrained to be a Lorentzian of width $\Gamma$ convoluted with the empirical elastic peak shape. The excitation energy of this peak and its area were allowed to vary. Its energy was found to be $9.00 \pm 0.18$ $\mathrm{MeV}$ with little variation from angle to angle.

The broad $0^{+}$and $2^{+}$states are known to have slightly different energies, and both were populated in ${ }^{10} \mathrm{~F}\left({ }^{3} \mathrm{He}, \mathrm{d}\right)$, giving a peak whose location and shape changed with angle. However, excited $0^{+}$states are typically very weak in pion inelastic scattering, and we would not expect to observe the $0^{+}$state strongly populated in our experiment. The constancy of the peak location with angle and the angular-distribution shape (as we note below) are consistent with the absence of appreciable $0^{+}$strength. Varying the width primarily affects the magnitude of the background and the areas of the narrow peaks between 8.5 and $9.5 \mathrm{MeV}$ in excitation. If we make $\Gamma$ too small or too large, we tend to increase the background or the fitted cross section to the narrow peaks. With $\Gamma=800 \mathrm{keV}$ we see no $2^{+}$component in the angular distributions of the narrow states except those known to have $\mathrm{J}^{\pi}=2^{+}$, and, as several of the known narrow states are not $2^{+}$, this implies we have approximately the correct magnitude and width for the $9.0-\mathrm{MeV}$ state.

Angular distributions for this broad peak are displayed in Figure 2, along with the angular distribution of the $1.63-\mathrm{MeV} 2_{1}^{+}$level (not shown in figure 1). Collective-model and microscopic calculations have been performed using the codes $\mathrm{NDWPI}^{\circ}$ and $\mathrm{HL}^{7}$, respectively. The parameters for the neutron and proton ground-state densities, and for the collective transition density, were all taken to be equal to the proton density measured in electron scattering ${ }^{5}$. The collectivemodel calculations reproduce reasonably well the first maximum and minimum of the $1.63-\mathrm{MeV}$ angular distribution, but the fit is not as good for the $9.0-\mathrm{MeV}$ state. 
In order to get a better fit it is necessary to increase the radius of the transition density, by up to $25 \%$ over that for the $1.63 \mathrm{-MeV}$ state. From this procedure we find $B(E 21)$ is between 25 and $35 \mathrm{e}^{2} \mathrm{fm}^{4}$ for the $9.0-\mathrm{MeV}$ state and $323 \mathrm{e}^{2} \mathrm{fm}$ for the 1.63-MeV state. The transition between the $9.0-\mathrm{MeV}$ state and the ground state has not been seen previously. The latest compilation ${ }^{1}$ gives $B(E 21)=335 \pm 20 e^{2} \mathrm{fm}^{4}$ for the trangition from the ground state the $1.63-\mathrm{MeV}$ state.

The larger radius of the transition density is consistent with the belief that the $\mathrm{K}^{\pi}=0_{4}^{+}$band involves the excitation of either two or four particles into the $f p$ shell. Results of calculations using microscopic transition densities are displayed in Figure 3. We have used a $1 \mathrm{~d} 5 / 2-1 \mathrm{~d} 5 / 2$ and a $1 \mathrm{f7} / 2-1 \mathrm{f} 7 / 2$ transition density and arbitrarily normalized the calculations to the data. While both calculations fit the data, the shift in the minima between the $d 5 / 2$ and $f 7 / 2$ calculations is similar to the shift between the $1.63-\mathrm{MeV}$ angular distribution and the $9.0-\mathrm{MeV}$ angular distribution. Of course, the ground state of ${ }^{20} \mathrm{Ne}$ contains very few $f 7 / 2$ nucleons, so this is not a realistic calculation. Furthermore, an $(\mathrm{fp})^{4}$ or $(\mathrm{fp})^{2}(\mathrm{sd})^{2} 2^{+}$state can not be reached from a predominantly (sd) ${ }^{420} \mathrm{Ne}$ (g.s.) by a one-body E2 operator. For this reason, the large $B(E 2)$ resulting from the collective-model analysis may be difficult to explain. The broad $0^{+}$and $2^{+}$states are thought ${ }^{3,4}$ to mix with nearby (sd) ${ }^{4}$ states, but that $2^{+}$level (at $7.42 \mathrm{MeV}$ ) has only a weak ground state $\mathrm{B}(\mathrm{E} 2)$.

Our resulto may imply mixing between these broad states and the first two members of the ground state band. Let $\phi_{z}$ and $\psi_{z}$ be the (sd) configuration which dominates the ground-state band and $\phi_{e}$ and $\psi_{0}$ be the $(f p)^{4}$ or $(f p)^{2}(s d)^{2}$ which is expected to dominate in the broad states. Then we can write

$$
\Phi_{0.0}=\mathrm{a} \phi_{8}+\mathrm{b} \phi_{.} \text {and } \Phi_{8.3}=-\mathrm{b} \phi_{\mathrm{z}}+\mathrm{a} \phi_{.}
$$


for the $0^{+}$states in the bands and

$$
\Psi_{1.03}=A \psi_{g}+B \psi_{e} \text { and } \Psi_{9.0}=-B \psi_{g}+A \psi_{e}
$$

for the $2^{+}$members of the bands, where

$$
\mathrm{A}^{2}+\mathrm{B}^{2}=1 \text { and } \mathrm{a}^{2}+\mathrm{b}^{2}=1
$$

For the transitions we define

$$
\left\langle\psi_{\mathrm{g}}|| \mathrm{M}(\mathrm{E} 2)|| \phi_{\mathrm{g}}\right\rangle=\mathrm{u} \text { and }\left\langle\psi_{\mathrm{e}}|| \mathrm{M}(\mathrm{E} 2)|| \phi_{\mathrm{e}}\right\rangle=\mathrm{v}
$$

with the cross transitions zero. Finally, for the matrix elements of the potential that causes the mixing,

$$
V_{0}=\left\langle\phi_{e}|V| \phi_{8}\right\rangle \text { and } V_{2}=\left\langle\psi_{e}|V| \psi_{8}\right\rangle \text {, }
$$

we assume $V_{2}=\frac{1}{\sqrt{5}} V_{0}$. With these assumptions, our measured $B(E 2)$ 's can be used to extract $V_{0}$ (or $B$ ) as a function of $v / u$. These are plotted in Figure 4. We see that a large mixing matrix element, at least $1 \mathrm{MeV}$, is necessary to explain the data. Remember that to account for the proton transfer data of references 3 and 4 , it was necessary to include the $6.73-\mathrm{MeV} \mathrm{O}^{+}$and $7.42-\mathrm{MeV}^{+}$states in the mixing. The mixing deduced here for the B(E2)'s will not reprcduce the observed $\left({ }^{3} \mathrm{He}, \mathrm{d}\right)$ results. Thus, obviously any two-state model is too restrictive, but a matrix element of the derived magnitude is not unreasonable

In summary, we have seen the $2^{+}$member of the $\mathrm{K}^{\pi}=\mathrm{O}_{4}^{+}$band of ${ }^{20} \mathrm{Ne}$ in pion inelastic scattering. We have measured its energy and width to be $9.00 \pm 0.18$ $\mathrm{MeV}$ and $800 \mathrm{keV}$, respectively. We have also determined $\mathrm{B}(\mathrm{E} 2 \mathrm{l})$ to be approximately 25 to $35 \mathrm{e}^{2} \mathrm{fm}^{4}$ for the trangition from the ground state. Our data suggest that the transition density for this state extends to a larger radius than for the 1.63-MeV $2^{+}$level. 
-225 -

This work was supported in part by the United States Department of Energy, the Robert A. Welch Foundation, and the National Science Foundation. 


\section{References}

- Present address: Bates Linear Accelerator, Massachusetts Institute of Technology, Cambridge, MA 02139

'Present address: George Washington University, Washington, DC 20052

1. F. Ajzenberg-Selove, Nucl. Phys. A475, 1 (1987).

2. L. C. McDermott, K. W. Jones, H. Smotrich, and R. E. Benenson, Phys. Rev. 118,175 (1960).

3. R. R. Betts, H. T. Fortune, and R. Middleton, Pbys. Rev. C11, 19 (1975).

4. H. T. Fortune, R. R. Betts, and R. Middleton, Phys. Lett. 62B, 287 (1976).

5. G. Rowe, M. Salomon, and R. H. Landau, Phys. Rev. C18, 584 (1978).

6. R. A. Eisenstein and G. A. Miller, Computer Physics Communications 11, 95 (1976).

7. T.-S. H. Lee and D. Kurath, Phys. Rev. C21, 293 (1980).

8. H. DeVries, C. W. DeJager, and C. DeVries, Atomic Data and Nuclear Data Tables 36, 495 (1987). 
-227 -

Table I Parameters of the $\mathrm{O}_{4}^{+}, 2_{4}^{+}$states in ${ }^{20} \mathrm{Ne}$.

\begin{tabular}{ccccc}
\hline \multirow{2}{*}{ Reaction } & \multicolumn{2}{c}{$0^{+}$} & \multicolumn{2}{c}{$2^{+}$} \\
\cline { 2 - 5 } & $\mathrm{E}_{\mathbf{x}}(\mathrm{MeV})$ & $\Gamma(\mathrm{keV})$ & $\mathrm{E}_{\mathbf{x}}(\mathrm{MeV})$ & $\Gamma(\mathrm{keV})$ \\
\hline \hline${ }^{10} \mathrm{O}(\alpha, \alpha)^{\mathbf{2}}$ & $\approx 8.7$ & $>800$ & 8.8 & $>800$ \\
${ }^{10} \mathrm{~F}\left({ }^{3} \mathrm{He}, \mathrm{d}\right)^{b, c}$ & $\approx 8.3$ & $\approx 800$ & $\approx 8.8$ & - \\
${ }^{20} \mathrm{Ne}\left(\pi, \pi^{\prime}\right)^{\mathrm{d}}$ & - & - & $9.00 \pm 0.18$ & $\approx 800$
\end{tabular}

ref. 2.

b ref. 3.

c ref. 4.

d present work. 


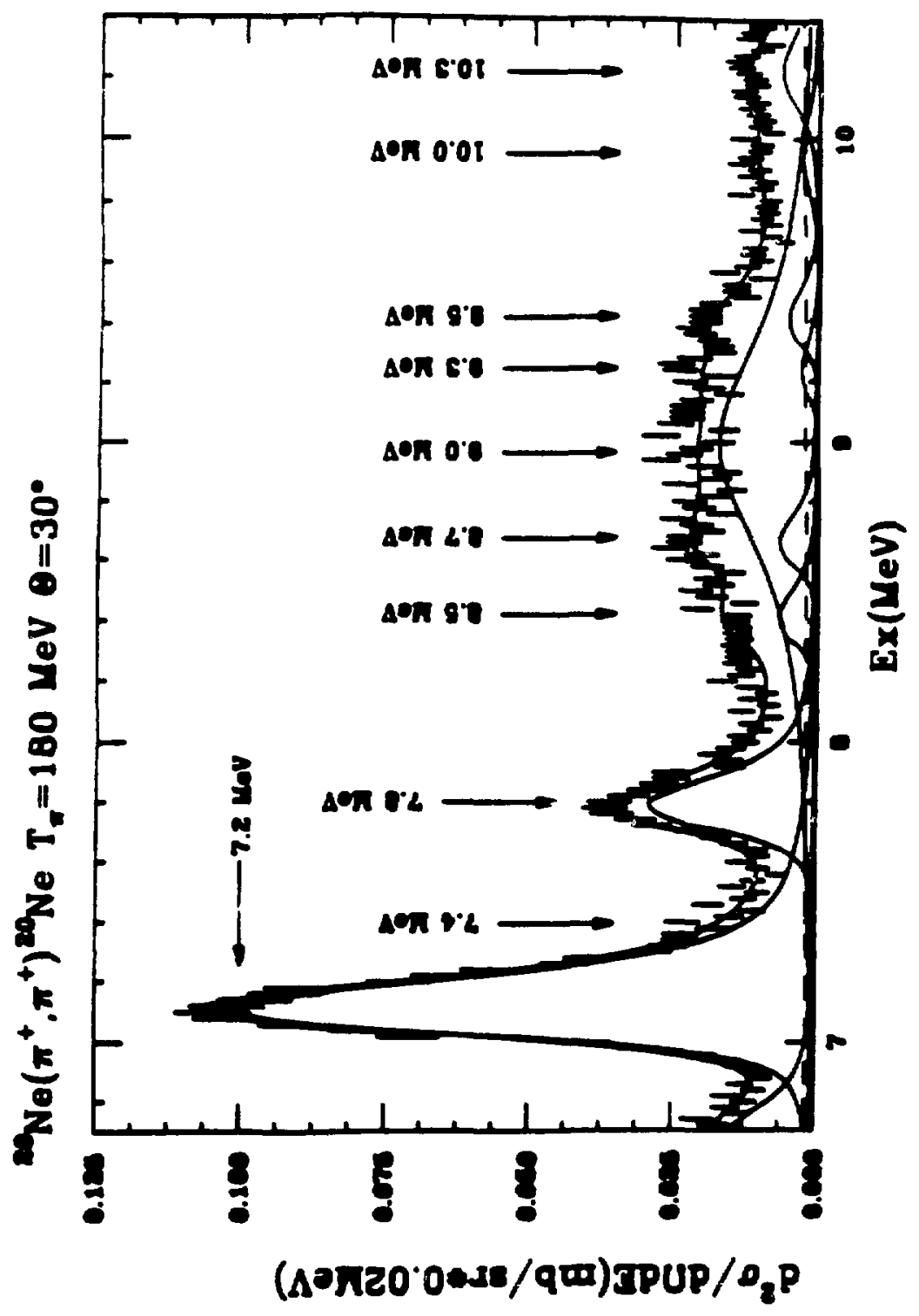

Fig. 1

Fitted missing mass spectrum for $180 \mathrm{MeV} \pi^{+}$scattering from ${ }^{20}$ Ne at $30^{\circ}$. 


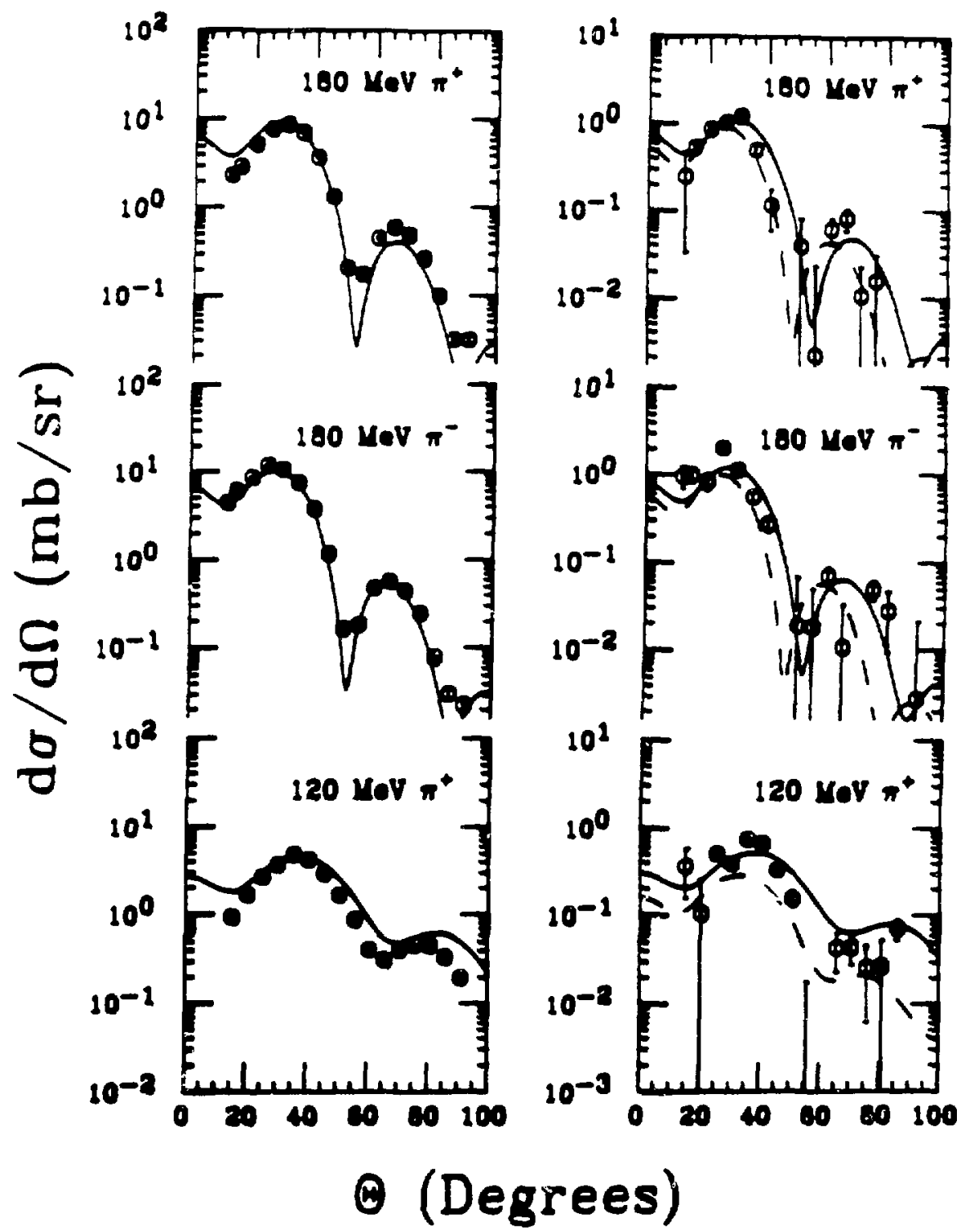

Fig. 2

Angular distribution for "Ne( $\pi, \pi$ ') to the $2^{+}$levels at $1.63 \mathrm{MeV}$ and $9.0 \mathrm{MeV}$. The curree are resulte of collectire-model calculations discused in the text. The solid curve use parameters for the transition density taken from elastic electron scattering. The dashed curre use a iransition density with a larger transition radius. 


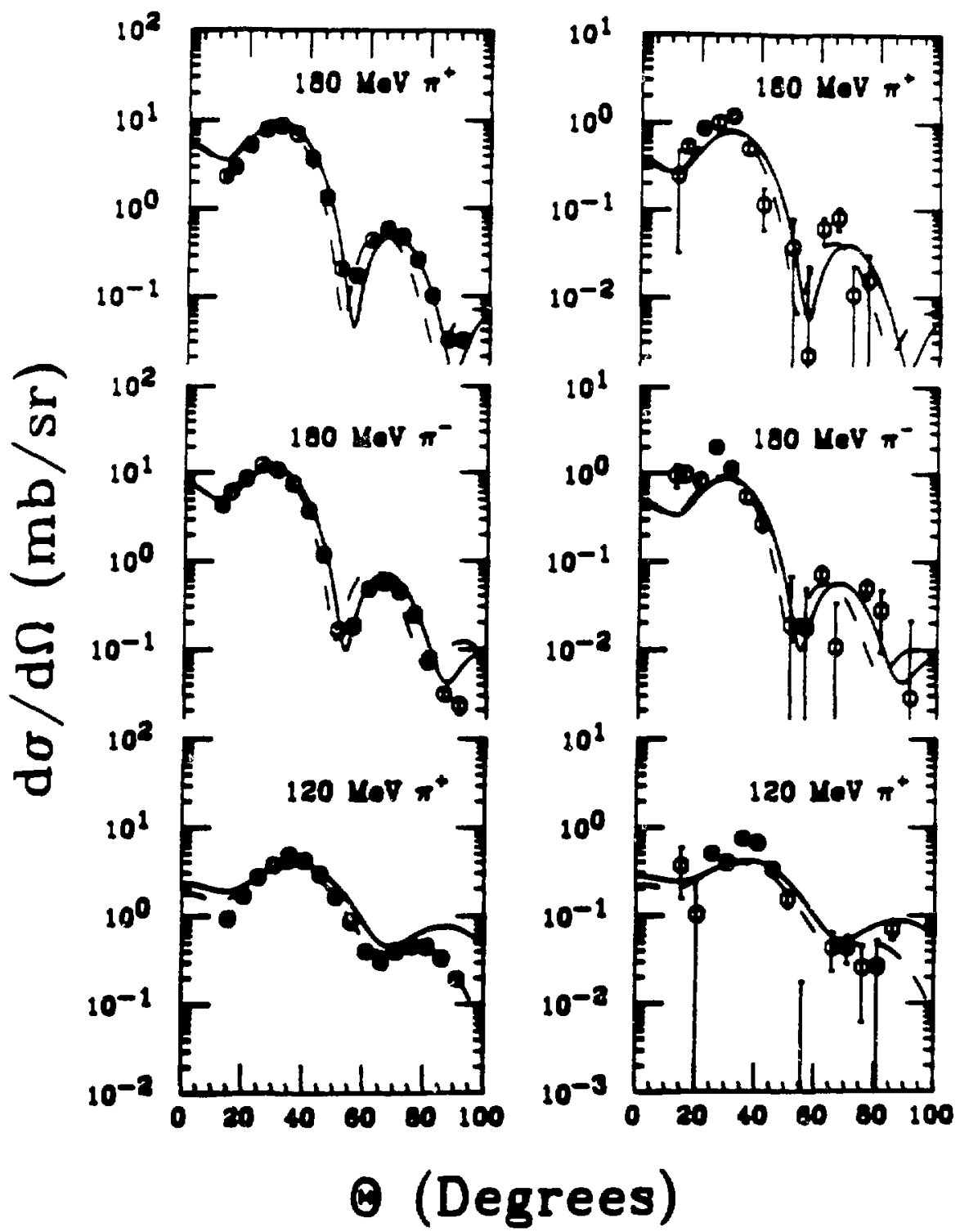

Fig. 3

Data from figure 2, compared with result of microscopic calculations. The solid curre is for $1 \mathrm{~d} 5 / 2-1 \mathrm{d5} / 2$ and the dashed curre is for $177 / 2-17 / 2$ as discussed in the text. 


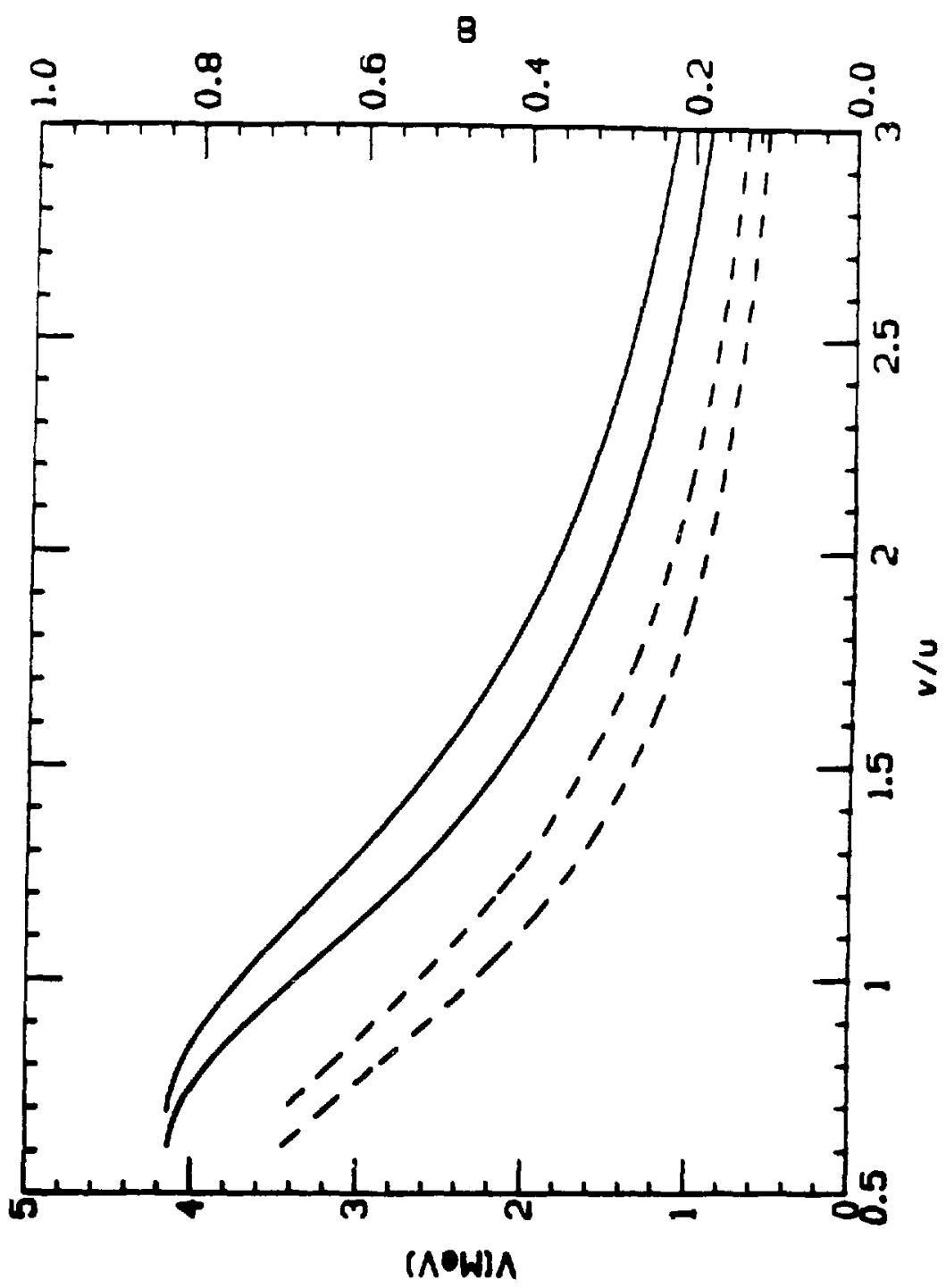

Fic. 4

The mixing potential and $B$ a a function of $v / u$ for the two-state model described in the text. The solid curre is $V_{0}$ and the dashed curre ia $B$. The lower curve in each case is for $B(E 21)=25 e^{2} \mathrm{fm}^{4}$ and the upper curve for $B(E 21)=35 e^{2} \mathrm{fm}$. 


\section{Bibliography}

AJ-87 F. Ajzenberg-Selove, Nucl. Phys. A475, 1 (1987)

AL-72 T.K. Alexander, et al., Nucl. Phys. A197, 1 (1972)

AL-85 T. K. Alexander, B. Castel, and I. S. Towner, Nucl. Phys. A445, 189 (1985)

AM-88 W. M. Amos, Senior Honor's Thesis, University of Pennsylvania, (1988)

AU-71 N. Auerbach, Phys. Lett. 36B, 293 (1971)

BA-72 D. P. Balamuth, J. W. Noe', H. T. Fortune, and R. W. Zurmunle, Phys. Rev. C6, 1694 (1972)

BA-86 F. Balestra, et al., Nucl. Phys. A452, 573 (1986)

BE-72 G. F. Bertsch, The Practitioner's Shell Model, (American Elsevier Publishing Company, Inc, 1972)

BE-75 R. R. Betts, H. T. Fortune, and R. Middleton, Phys. Rev. C11, 19 (1975)

BL-84a L. Bland, Thesis, University of Pennsylvania, Los Alamos Scientific Report No. LA-9960-T, (1984)

BL-84b G. S. Blanpied, et al., Phys. Rev. C30, 1233 (1984)

BL-88 G. S. Blanpied, et al., Phys. Rev. C38, 2180 (1988)

BR-77 P. J. Brussard and P. W. M. Glaudemans, Shell-Model Applications in Nuclear Spectroscopy, (Nortb-Holland Publishing Company, 1977)

BR-82 B. A. Brown, et al., Phys. Rev. C20, 2247 (1882)

BR-83 B. A. Brown, R. Radhi, and B. H. Wildenthal, Phys. Rep. 101, 313 (1983)

CO-01 J. R. Comfort, private communication 
C0-72 J. R. Comfort, et al., Phys. Lett. 40B, 456 (1972)

CO-80 W. B. Cottingame and D. B. Holtkamp, Phys. Rev. Lett. 45, 1828 (1980)

CR-74 D. J. Crozier and H. T. Fortune, Phys. Rev. C10, 1697 (1974)

DE-73 T. J. Deal, Nucl. Phys. A217, 210 (1973)

DE-87 H. DeVries, C. W. DeJager, and C. DeVries, Atomic and Nucl. Data Tables, 36, 495 (1987)

DI-71 W. T. Diamond, T. K. Alexander, and O. Hzusser, Can. J. Phys. 49, 1589 (1971)

ED-71 G. Edwards and E. Rost, Phys. Rev. Lett. 26, 785 (1971)

EI-62 W. W. Eidson and R. D. Bent, Phys. Rev. 128, 1312 (1962)

EI-74 R. A. Eisenstein and G. A. Miller, Computer Physics Communications 8, $130(1974)$

EI-76a R. A. Eisenstein and G. A. Miller, Computer Physics Communications $11,95(1976)$

EI-76b R. A. Eisenstein and F. Tabakin, Computer Physics Communications 12, 237 (1976)

ER-88a D. J. Erast and K. S. Dhuga, Phys. Rev. C37, 2651 (1988)

ER-88b D. J. Ernst, Pion-Nucleus Physics: Future Directions and New Facilitie at LAMPF, R. J. Peterson and D. D. Strottman, editors, (AIP Conference Proceedings 163) p. 513ff (1988)

FA-71 W. R. Falk, R. Kulisic, and A. McDonald, Nucl. Phys. A167, 157 (1971)

FO-72a H. T. Fortune, R. Middleton, and R. R. Betts, Phys. Rev. Lett. 29, 738 (1972) 
FO-72b H. T. Fortune, Nuclear Spectroscopy with Heavy Ion Reactions, (Proceedings of the Heavy Ion Summer Study Oak Ridge National Laboratory CONF-720669), S. T. Thornton editor, pp. 353-377 (1972)

F0-73a H. T. Fortune, Heavy Ion Lecture Series Vol. 2, (Kansas State University 1972-1973), J. D. Spangler and G. G. Seaman, editors, pp 1$132(1973)$

FO-73b H. T. Fortune, R. R. Betts, J. D. Garrett, and R. Middleton, Phys. Lett. 44B, 65 (1973)

FO-74a H. T. Fortune, R. R. Betts, and R. Middleton, Phys. Rev. C10, 2135 (1974)

FO-74b H. T. Fortune, L. R. Greenwood, R. E. Segel, and J. R. Erskine, Phys. Lett. 62B, 51 (1974)

FO-76 H. T. Fortune, R. R. Betts, and R. Middleton, Phys. Lett. 62B, 282 (1976)

FO-77 H. T. Fortune, J. Phys. Soc. Jap. 44 Suppl., 99 (1978)

FO-78 H. T. Fortune, et al., Phys. Rev. C17, 1955 (1978)

GL-83 N. Glendenning, Direct Nuclear Resctions, (Academic Press, 1983)

HA-68 M. Harvey, Advances in Nuclear Physics 1, 67 (1968)

HA-72 O. HRusser, et al., Nucl. Phys. A179, 465 (1972)

HA-81 M. N. Harakeh and A. E. L. Dieperink, Phys Rev C23, 2329 (1981)

HE-72 D. W. Heikkinen, H. F. Lutz, and W. Batolini, Nucl. Phys. A193, 372 (1972)

HI-77 M. Hirata, F. Lenz, and K. Yazaki, Annals of Phys. 108, 116 (1977) 
HO-70 A. J. Howard, J. G. Pronko, and R. G. Hirko, Nucl. Phys. A150, 609 (1970)

KE-59 A. Kerman, H. McManus, and R. Thaler, Annals of Phys. 8, 551 (1959)

KI-55 L. S. Kisslinger, Phys. Rev 98, 761 (1955)

KI-74 L. S. Kisslinger and F. Tabakin, Phys. Rev. C9, 188 (1974)

KO-65 J. Kokume, K. Fukunaga, H. Nakamura, and N. Inoue, J. Phys. Soc. Jap. $20,475(1965)$

KU-01 P. D. Kunz, private communication

LA-80 R. D. Lawson, Theory of the Nuclear Shell Model, (Oxford University Press, 1980)

LE-74 T.-S. H. Lee and F. Tabakin, Nucl. Phys. A226, 253 (1974)

LE-80a T.-S. H. Lee and R. D. Lawson, Phys. Rev. C21, 679 (1980)

LE-80b T.-S. H. Lee and D. Kurath, Phys. Rev. C21, 293 (1980)

LE-80c F. Lenz, E. J. Moniz, and K. Yazaki, Ann. Phys. 129, 84 (1980)

LE-82 F. Lenz, M. Thies, and Y Horikawa, Ann. Phys. 140, 266 (1982)

LI-67 A. Litherland, et al., Can. J. of Phys. 45, 1901 (1967)

LI-83 L. C. Liu, Phys. Rev. C27, 1611 (1983)

LO-74 J. T. Londergan, K. W. McVoy, and E. J. Moniz, Annals of Phys. 88, 147 (1974)

MC-60 L. C. McDermott, K. W. Jones, H. Smotrich, and R. F. Benenson, Phys. Rev. 118, 175 (1960)

MC-73 J. B. McGrory and B. H. Wildenthal, Phys. Rev. C7, 974 (1973)

ME-75 L. R. Medsker, H. T. Fortune, R. R. Betts, and R. Middleton, Phys. Rev. C11, 1880 (1975) 
MI-71 R. Middleton, J. D. Garrett, and H. T. Fortune, Phys. Rev. Lett. 27, 950 (1971)

MI-72 S. Mitsunobu and Y. Torizuka, Pbys. Rev. Lett. 28, 920 (1972)

MO-01 C. L. Morris, private communication

MO-02 C. L. Morris, private communication

MO-87 S. Mordechai et al., Phys. Rev. C36, 1950 (1987)

NI-55 S. G. Nilsson, K. Danske Vidensk. Selsk. Mat.-Fys. Medd 29, No. 16 (1955)

OA-87 D. S. Oakley, Thesis, University of Texas at Austin, Los Alamos Scientific Report No. LA.10968-T, (1987)

OD-59 Y. Oda, M. Tukeda, C. Hu, and S. Kato, J. Phys. Soc. Jap. 14, 396 (1959)

OD-60 Y. Oda, et al., J. Phys. Soc. Jap. 15, 760 (1960)

OX-85 B. A. Brown, A. Etchegoyen, and W. D. M. Rae, OXBASH computer code, (1985)

PA-86 Particle Data Group, Phys. Lett. 170B, 1 (1986)

PE-64 J. D. Pearson and R. H. Spear, Nucl. Phys. 54, 434 (1964)

PI-78 A. A. Pilt and H. T. Fortune, Lettere AL Nuovo Cimento 21, 502 (1978)

PR-75 M. A. Preston and R. K. Bhaduri, Structure of the Nucleus, (1975)

RE-71 H. Rebel, et al., Phys. Rev. Lett. 26, 1190 (1971)

RE-72 H. Rebel, et al., Nucl. Phys. A182, 145 (1972)

RO-01 E. Rost, private communication

RO-71a D. W. O. Rogers, et al., Phys. Lett. 37B, 65 (1971)

RO-71b D. W. O. Rogers, et al., Can. J. of Phys. 49, 1397 (1971) 
RO-78 G. Rowe, M. Salomon, and R. H. Landau, Phys. Rev. C18, 584 (1978)

SC-62 G. Schrank, E. K. Warburton, and W. W. Daebnick, Phys Rev. 127, $2159(1962)$

SE-58 L. Seidlitz, E. Bleuler, and D. J. Tendam, Phys. Rev. 110, 682 (1958)

SE-81 S. J. Seestrom-Morris, Thesis, University of Minnesota, Los Alamos Report No. LA-8916-T, (1981)

SH-74 A. de Shalit and H. Feshbach, Theoretical Nuclear Physica, vol. 1 Nuclear Structure (1974)

SI-73 R. P. Singhal, H. S. Caplan, J. R. Moreira, and T. E. Walker, Can. J. of Phys. 51, 2125 (1973)

SI-81 E. R. Siciliano and G. E. Walker, Phys. Rev. C23, 2661 (1981)

SP-65 A. Springer and B. G. Harvey, Phys. Rev. Lett. 14, 316 (1965)

SP-70 J. Specht, et al., Nucl. Phys. A143, 373 (1970)

ST-70 P. Strehl, Z. Phys. 234, 416 (1970)

SW-69 R. de Swiniarski, et al., Phys. Rev. Lett. 23, 317 (1969)

SW-73 R. de Swiniarski, et al., Phys. Lett. 43B, 27 (1973)

SW-74 R. de Swiniarski, A. Genoux-Lubain, G. Bagieu, and J. F. Cavaignac, Can. J. of Phys. 52, 2422 (1974)

SW-76 R. de Swiniarski, F. G. Resmini, D. L. Hendrie, and A. D. Bacher, Nucl. Phys. A261, 111 (1976)

WI-84 B. H. Wildenthal, Prog. in Part. and Nucl. Phys. 11, 5 (1984)

ZU-01 J. D. Zumbro, private communication

ZU-68 A. P. Zuker, B. Buck, and J. B. McGrory, Phys. Rev. Lett. 21, 39 (1968) 